\section{SANDIA REPORT}

SAND98-2709

Unlimited Release

Printed December 1998
MS-0619

Review \& Approval Desk, 15102

For DOE/OSTI

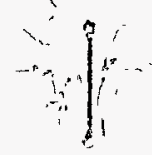

\title{
A Family of Uniform Strain Tetrahedral Elements and a Method for Connecting
}

\section{Dissimilar/Finite Element Meshes}

C. R. Dohrmann, S. W. Key, M. W. Heinstein, J. Jung

\section{Frepared by}

Sandia National Laboratories

Albuquerque, New Mexico 87185-ańd Livermore, Califomia 94550

Sandia is a multiprogram laboratory operated by Sandia Corporation,

a Lockheed Martin Company, for the United States Department of

Energy under Contract DE-AC04-94AL85000.

Approved for public release; further dissemination unlimited. 
Issued by Sandia National Laboratories, operated for the United States Department of Energy by Sandia Corporation.

NOTICE: This report was prepared as an account of work sponsored by an agency of the United States Government. Neither the United States Government nor any agency thereof, nor any of their employees, nor any of their contractors, subcontractors, or their employees, makes any warranty, express or implied, or assumes any legal liability or responsibility for the accuracy, completeness, or usefulness of any information, apparatus, product, or process disclosed, or represents that its use would not infringe privately owned rights. Reference herein to any specific commercial product, process, or service by trade name, trademark, manufacturer, or otherwise, does not necessarily constitute or imply its endorsement, recommendation, or favoring by the United States Government, any agency thereof, or any of their contractors or subcontractors. The views and opinions expressed herein do not necessarily state or reflect those of the United States Government, any agency thereof, or any of their contractors.

Printed in the United States of America. This report has been reproduced directly from the best available copy.

Available to DOE and DOE contractors from

Office of Scientific and Technical Information

P.O. Box 62

Oak Ridge, TN 37831

Prices available from (615) 576-8401, FTS 626-8401

Available to the public from

National Technical Information Service

U.S. Department of Commerce

5285 Port Royal Rd

Springfield, VA 22161

NTIS price codes

Printed copy: A07

Microfiche copy: A01

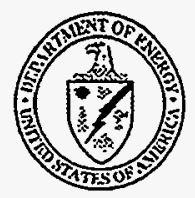


SAND98-2709

Unlimited Release

Printed December 1998

\title{
A Family of Uniform Strain Tetrahedral Elements and a Method for Connecting Dissimilar Finite Element Meshes
}

\author{
C. R. Dohrmann \\ Structural Dynamics Department \\ S. W. Key, M. W. Heinstein, J. Jung \\ Engineering and Manufacturing Mechanics Department \\ Sandia National Laboratories \\ P.O. Box 5800 \\ Albuquerque, NM 87185-0439
}

\begin{abstract}
This report documents a collection of papers on a family of uniform strain tetrahedral finite elements and their connection to different element types. Also included in the report are two papers which address the general problem of connecting dissimilar meshes in two and three dimensions. Much of the work presented here was motivated by the development of the tetrahedral element described in the report "A Suitable Low-Order, Eight-Node Tetrahedral Finite Element For Solids," by S. W. Key et al., SAND98-0756, March 1998. Two basic issues addressed by the papers are: (1) the performance of alternative tetrahedral elements with uniform strain and enhanced uniform strain formulations, and (2) the proper connection of tetrahedral and other element types when two meshes are "tied" together to represent a single continuous domain.
\end{abstract}




\section{Executive Summary}

The unavailability of a robust, automated, all-hexahedral mesher motivated recent investigations of a family of uniform strain tetrahedral elements [1-2]. These elements were shown to posses the same convergence and antilocking characteristics of the uniform strain hexahedron. A related study of enhanced versions of these elements [3] was also carried out. It was shown that significant improvements in accuracy are obtained for certain element types by allowing more than a single state of uniform strain within each element.

An important advantage of the tetrahedron over the hexahedron is its ability to more readily mesh complicated geometries. On the other hand, more tetrahedral elements are generally required to mesh a volume for a specified element edge length. Taking these factors into consideration, a transition element was developed for meshes containing both hexahedral and tetrahedral elements [4]. This effort was motivated by the idea of meshing a geometry primarily with hexahedral elements. For regions of the mesh that cannot be completed with hexahedral elements, a direct transition to tetrahedral elements could be made to complete the mesh. In this way, the advantages of both element types could be brought to bear on the meshing problem.

The development of the transition element in Ref. 4 lead naturally to a general method for connecting dissimilar finite element meshes in two and three dimension [5-6]. The method combines the concept of master and slave surfaces with the uniform strain approach for finite elements. By modifying the boundaries of elements on the slave surface, corrections are made to element formulations such that first-order patch tests are passed. The method can be used to connect meshes which use different element types. In addition, master and slave surfaces can be designated independently of relative mesh resolutions. It was shown that significant improvements in accuracy, especially at the shared boundary, are obtained using the new approach compared with standard approaches used in existing finite element codes.

The purpose of this report is to provide a single document for the work presented in Refs. 2-6. The first two papers deal specifically with the development and performance of a family of uniform strain tetrahedral elements. The third paper shows how to properly connect tetrahedral elements to the faces of hexahedral elements. The final two papers identify and explore the 
implementation of the definitive requirement which must be satisfied when two separately meshed regions are tied together. For two meshes to be tied together properly, the volume both initially and generated during subsequent deformation must be computed exactly, added to the finite elements on one side of the interface, and incorporated into the finite elements' mean-stress gradient/divergence operator.

\section{References}

1. S. W. Key, M. W. Heinstein, C. M. Stone, F. J. Mello, M. L. Blanford and K. G. Budge, 'A Suitable Low-Order, 8-Node Tetrahedral Finite Element for Solids', accepted for publication in International Journal for Numerical Methods in Engineering.

2. C. R. Dohrmann, S. W. Key, M. W. Heinstein and J. Jung, 'A Least Squares Approach for Uniform Strain Triangular and Tetrahedral Finite Elements', International Journal for Numerical Methods in Engineering, 42, 1181-1197 (1998).

3. C. R. Dohrmann and S. W. Key, 'Enhanced Uniform Strain Triangular and Tetrahedral Finite Elements,' submitted to International Joumal for Numerical Methods in Engineering.

4. C. R. Dohrmann and S. W. Key, 'A Transition Element for Uniform Strain Hexahedral and Tetrahedral Finite Elements,' accepted for publication in International Journal for Numerical Methods in Engineering.

5. C. R. Dohrmann, S. W. Key and M. W. Heinstein, 'A Method for Connecting Dissimilar Finite Element Meshes in Two Dimensions', submitted to International Journal for Numerical Methods in Engineering.

6. C. R. Dohrmann, S. W. Key and M. W. Heinstein, 'A Method for Connecting Dissimilar Finite Element Meshes in Three Dimensions', submitted to International Journal for Numerical Methods in Engineering. 


\title{
A Least Squares Approach for Uniform Strain Triangular and Tetrahedral Finite Elements ${ }^{1}$
}

\author{
C. R. Dohrmann ${ }^{2}$ \\ S. W. $\mathrm{Key}^{3}$ \\ M. W. Heinstein ${ }^{3}$ \\ J. Jung ${ }^{3}$
}

\begin{abstract}
A least squares approach is presented for implementing uniform strain triangular and tetrahedral finite elements. The basis for the method is a weighted least squares formulation in which a linear displacement field is fit to an element's nodal displacements. By including a greater number of nodes on the element boundary than is required to define the linear displacement field, it is possible to eliminate volumetric locking common to fullyintegrated lower-order elements. Such results can also be obtained using selective or reduced integration schemes. but the present approach is fundamentally different from those. The method is computationally efficient and can be used to distribute surface loads on an element edge or face in a continuously varying manner between vertex, mid-edge and mid-face nodes. Example problems in two and three-dimensional linear elasticity are presented. Element types considered in the examples include a six-node triangle, eight-node tetrahedron, and ten-node tetrahedron.
\end{abstract}

Key Words. Finite elements, least squares, uniform strain, hourglass control.

\footnotetext{
${ }^{1}$ Sandia is a multiprogram laboratory operated by Sandia Corporation, a Lockheed Martin Company, for the United States Department of Energy under Contract DE-AL0494AL8500.

${ }^{2}$ Structural Dynamics Department, Sandia National Laboratories, MS 0439, Albuquerque, New Mexico 87185-0439, email: crdohrm@sandia.gov, phone: (505) 844-8058, fax: (505) 844-9297.

${ }^{3}$ Engineering and Manufacturing Mechanics Department, Sandia National Laboratories, MS 0443, Albuquerque, New Mexico 87185-0443.
} 


\section{Introduction}

Constant strain finite elements such as the three-node triangle and the four-node tetrahedron are easily formulated, but their performance in applications is often unsatisfactory. The poor performance of these elements is most notable for incompressible or nearly incompressible materials. For such materials, the effects of volumetric locking render the elements overly stiff. Similar characteristics are exhibited by fully-integrated lower-order elements such as the four-node quadrilateral and the eight-node hexahedron.

Selective and reduced integration have been shown to be effective methods for reducing the overly stiff behavior of lower-order elements. The basic idea with such approaches is to integrate the strain energy of the element in an approximate sense. By doing so, the element becomes more flexible. Such approaches typically require the calculation of shape function gradients and are element specific. Moreover, special care must be taken to ensure that the method of quadrature correctly assesses states of uniform stress and strain [1].

The present approach departs from methods of selective or reduced integration in two important respects. First, a linear displacement field is assumed within each element at the outset. As a result, element strains are constant and the strain energy is integrated exactly. Secondly, the equations used to calculate strains and hourglass deformations only depend on the nodal coordinates and displacements. Information concerning the shape functions used in the element formulation is not required.

The basis for the approach is a weighted least squares formulation in which a linear displacement field is fit to an element's nodal displacements. If the number of nodes equals the minimum required to define the displacement field (three in 2D and four in 3D), then the element simplifies to a traditional constant strain element. In this case, the fitted linear displacement field evaluated at the nodal coordinates is equal to the nodal displacements. For elements with nodes in excess of this number, the assumed linear displacement field and nodal displacements need not be consistent. This feature of the element gives it the flexibility required to overcome the shortcomings of traditional constant strain elements.

As the reader may have ascertained, the least squares approach does not explicitly make use of conventional shape functions that interpolate the nodal displacements. Although different in origin, the benefits gained by such an approach are the same as those for selective or reduced integration. That is, the element stiffness is effectively reduced. In the limit as a mesh is refined to greater and greater extent, the approximations introduced by the present approach become insignificant because constant strain elements can adequately approximate the exact solution. Convergence of the element types considered in this study follows from the satisfaction of patch tests $A$ through $C$ given in Zienkiewicz [2].

Because the approach is essentially an assumed strain method, certain conditions must 
be satisfied in order for it to have a variational justification [3]. These conditions along with an alternative mean quadrature approach are discussed in the Appendix. The conditions under which the two approaches are equivalent along with a method for ignoring certain mid-face or mid-edge nodes are also discussed. The ability to ignore certain nodes in the element formulation may prove useful for applications involving contact and for meshes with different element types, e.g., meshes with both uniform strain hexahedral and tetrahedral elements.

An interesting feature of the triangular and tetrahedral elements developed here is their ability to distribute surface loads on an element edge or face in a continuously varying manner between vertex, mid-edge and mid-face nodes. To illustrate, consider a bar of constant cross section modeled with ten-node tetrahedral elements. The ends of the bar are displaced to result in a state of uniaxial stress. Depending on the weights chosen in the least squares formulation, the distribution of reaction forces at the ends of the bar can vary from all at the vertex nodes to all at the mid-edge nodes.

The primary advantages of the uniform strain elements considered here over their fullyintegrated quadratic counterparts are computational efficiency and flexibility in distributing surface loads between vertex and mid-edge nodes. For example, a ten-node tetrahedral element with quadratic interpolation distributes a uniform pressure load entirely at the midedge nodes of a face. Such a distribution may not be desirable for applications involving contact.

Details of the present approach are provided in the following section. Example problems in 2D and 3D linear elasticity are given in Section 3. The uniform strain elements considered in the examples include a six-node triangle, eight-node tetrahedron, and ten-node tetrahedron. The same element formulation is used for all the element types mentioned.

\section{Element Formulation}

Consider a generic finite element with nodal coordinates $\left(x_{i}, y_{i}, z_{i}\right)$ for $i=1, \ldots, n$. The displacement of node $i$ in the $X, Y$ and $Z$ coordinate directions is denoted by $u_{i}, v_{i}$ and $w_{i}$, respectively. Without loss of generality, the origin of the element coordinate system is located at the weighted geometric center. That is,

$$
\sum_{i=1}^{n} \hat{w}_{i} x_{i}=0, \quad \sum_{i=1}^{n} \hat{w}_{i} y_{i}=0, \quad \sum_{i=1}^{n} \hat{w}_{i} z_{i}=0
$$

where $\hat{w}_{1}, \ldots, \hat{w}_{n}$ are positive nodal weights. Let $u(x, y, z), v(x, y, z)$ and $w(x, y, z)$ denote the displacements of a material point with coordinates $(x, y, z)$. For purposes of calculating 
element strains, the following linear displacement field is assumed:

$$
\begin{aligned}
u(x, y, z) & =\epsilon_{x} x+\gamma_{x y} y+r_{x}+r_{x y} y-r_{z x} z \\
v(x, y, z) & =\epsilon_{y} y+\gamma_{y z} z+r_{y}+r_{y z} z-r_{x y} x \\
w(x, y, z) & =\epsilon_{z} z+\gamma_{z x} x+r_{z}+r_{z x} x-r_{y z} y
\end{aligned}
$$

where the $\epsilon$ 's and $\gamma$ 's are the constant normal and shear strains of the element and the $r$ 's are associated with rigid body translations and rotations.

The element formulation is based on a least squares fit of the linear displacement field to the nodal displacements. The least squares problem in 3D is formulated as follows:

$$
\text { minimize } \quad(\Phi q-d)^{T} W(\Phi q-d)
$$

where

$$
\begin{gathered}
q=\left[\begin{array}{llllllllllll}
\epsilon_{x} & \epsilon_{y} & \epsilon_{z} & \gamma_{x y} & \gamma_{y z} & \gamma_{z x} & r_{x} & r_{y} & r_{z} & r_{x y} & r_{y z} & r_{z x}
\end{array}\right]^{T} \\
d=\left[\begin{array}{llllllllll}
u_{1} & v_{1} & w_{1} & u_{2} & v_{2} & w_{2} & \ldots & u_{n} & v_{n} & w_{n}
\end{array}\right]^{T} \\
W=\operatorname{diag}\left(\hat{w}_{1}, \hat{w}_{1}, \hat{w}_{1}, \hat{w}_{2}, \hat{w}_{2}, \hat{w}_{2}, \ldots, \hat{w}_{n}, \hat{w}_{n}, \hat{w}_{n}\right)
\end{gathered}
$$

and

$$
\Phi=\left[\begin{array}{cccccccccccc}
x_{1} & 0 & 0 & y_{1} & 0 & 0 & 1 & 0 & 0 & y_{1} & 0 & -z_{1} \\
0 & y_{1} & 0 & 0 & z_{1} & 0 & 0 & 1 & 0 & -x_{1} & z_{1} & 0 \\
0 & 0 & z_{1} & 0 & 0 & x_{1} & 0 & 0 & 1 & 0 & -y_{1} & x_{1} \\
\vdots & \vdots & \vdots & \vdots & \vdots & \vdots & \vdots & \vdots & \vdots & \vdots & \vdots & \vdots \\
x_{n} & 0 & 0 & y_{n} & 0 & 0 & 1 & 0 & 0 & y_{n} & 0 & -z_{n} \\
0 & y_{n} & 0 & 0 & z_{n} & 0 & 0 & 1 & 0 & -x_{n} & z_{n} & 0 \\
0 & 0 & z_{n} & 0 & 0 & x_{n} & 0 & 0 & 1 & 0 & -y_{n} & x_{n}
\end{array}\right]
$$

Notice that $W$ is the weighting matrix used in the least squares fitting and $\Phi$ spans the space of linear displacements sampled at the nodes.

Differentiating the function to be minimized with respect to $q$, setting the result equal to zero, and solving the resulting expression for $q$ yields

$$
q=S d
$$

where

$$
S=\left(\Phi^{T} W \Phi\right)^{-1} \Phi^{T} W
$$

Although Eq. (11) implies an expensive inversion for $S$, it is possible to obtain a closed-form expression for $S$, which is given in the Appendix. This expression allows for the efficient 
implementation of the present approach in standard finite element codes. It can also be used to efficiently calculate the shape functions for element free Galerkin (EFG) approaches [4]. To illustrate the efficiency, the Cholesky decomposition of $\Phi^{T} W \Phi$ requires $12^{3} / 3$ floating point operations using a standard algorithm [5]. In contrast, the inversion of the same matrix using the method in the Appendix only requires 42 flops once the moments given by Eqs. (66-67) are known.

Following the development in [1], the nodal force vector $f_{\sigma}$ associated with the element stresses is given by

$$
f_{\sigma}=V B^{T} \sigma
$$

where $V$ is the element volume, $B$ is the first six rows of the matrix $S$

$$
B=S(1: 6,:)
$$

and $\sigma$ is a vector of Cauchy stresses defined as

$$
\sigma^{T}=\left[\begin{array}{llllll}
\sigma_{x x} & \sigma_{y y} & \sigma_{z z} & \sigma_{x y} & \sigma_{y z} & \sigma_{z x}
\end{array}\right]^{T}
$$

So-called hourglass control is included in the element formulation to remove spurious zero energy modes. In this study we only consider hourglass stiffness, but one could easily include hourglass damping for problems in dynamics. Hourglass stiffness is designed to provide restoring forces for any nodal displacements orthogonal to $\Phi$.

The nodal displacement vector $d$ can be expressed as

$$
d=\Phi \tilde{q}+\Phi_{\perp} q_{\perp}
$$

where $\Phi^{T} \Phi_{\perp}=0$ and the columns of $\Phi_{\perp}$ are assumed orthonormal. Premultiplying Eq. (15) by $\Phi^{T}$ and solving for $\tilde{q}$ yields

$$
\tilde{q}=\left(\Phi^{T} \Phi\right)^{-1} \Phi^{T} d
$$

Substituting Eq. (16) into Eq. (15) leads to

$$
\Phi_{\perp} q_{\perp}=\left[I-\Phi\left(\Phi^{T} \Phi\right)^{-1} \Phi^{T}\right] d
$$

The strain energy associated with hourglass stiffness is formulated as

$$
U_{h}=\epsilon V^{1 / 3} G_{h} q_{\perp}^{T} q_{\perp} / 2
$$

where $\epsilon$ is a positive scalar and $G_{h}$ is a material modulus. Substituting Eq. (17) into Eq. (18) leads to

$$
U_{h}=\epsilon V^{1 / 3} G_{h} d^{T}\left[I-\Phi\left(\Phi^{T} \Phi\right)^{-1} \Phi^{T}\right] d / 2
$$


Finally, the nodal force vector $f_{h}$ associated with hourglass stiffness is obtained by differentiating $U_{h}$ with respect to $d$. The result is

$$
f_{h}=\epsilon V^{1 / 3} G_{h}\left[I-\Phi\left(\Phi^{T} \Phi\right)^{-1} \Phi^{T}\right] d
$$

It follows from Eq. (20) that $f_{h}$ is orthogonal to $\Phi \tilde{q}$. In other words, hourglass stiffness does not cause any restoring forces if the nodal displacements are consistent with a linear displacement field, the desired result. We note that the hourglass control given by Eq. (20) is also applicable to other uniform strain elements such as the eight-node hexahedron.

The development thus far has been focused solely on $3 \mathrm{D}$ elements. Corresponding results for $2 \mathrm{D}$ elements are obtained simply by redefining $q, d, W$ and $\Phi$ as

$$
\begin{gathered}
q=\left[\begin{array}{llllll}
\epsilon_{x} & \epsilon_{y} & \gamma_{x y} & r_{x} & r_{y} & r_{x y}
\end{array}\right]^{T} \\
d=\left[\begin{array}{lllllll}
u_{1} & v_{1} & u_{2} & v_{2} & \ldots & u_{n} & v_{n}
\end{array}\right]^{T} \\
W=\operatorname{diag}\left(\hat{w}_{1}, \hat{w}_{1}, \hat{w}_{2}, \hat{w}_{2}, \ldots, \hat{w}_{n}, \hat{w}_{n}\right)
\end{gathered}
$$

and

$$
\Phi=\left[\begin{array}{cccccc}
x_{1} & 0 & y_{1} & 1 & 0 & y_{1} \\
0 & y_{1} & 0 & 0 & 1 & -x_{1} \\
\vdots & \vdots & \vdots & \vdots & \vdots & \vdots \\
x_{n} & 0 & y_{n} & 1 & 0 & y_{n} \\
0 & y_{n} & 0 & 0 & 1 & -x_{n}
\end{array}\right]
$$

In the finite element method, equivalent nodal forces for surface tractions are commonly obtained by integrating the product of the shape functions and the tractions over the loaded area. This procedure cannot be used with the least squares approach because shape functions are never introduced.

Two alternative options are available for determining equivalent nodal loads. The first involves subjecting a collection of elements to a constant state of stress. Equivalent nodal forces can then be determined from the calculated reaction forces. A second method, presented in the Appendix, makes use of a mean quadrature formulation that is equivalent to the least squares approach under certain conditions.

The six-node triangle is defined to have three vertex nodes and three mid-edge nodes as shown in Figure 1a. The nodal weights for the element are chosen as

$$
\left(\hat{w}_{1}, \ldots, \hat{w}_{6}\right)=(1-\alpha, 1-\alpha, 1-\alpha, 4 \alpha, 4 \alpha, 4 \alpha)
$$


where $\alpha \in[0,1]$ is a scalar weighting parameter. When $\alpha=1 / 5$, the weighting for each node is identical. Consider a surface traction of constant value applied to the edge shared by nodes 1,2 and 4 . The equivalent nodal forces are given by

$$
\begin{gathered}
f_{1}=(1-\alpha) F / 2, \quad f_{2}=(1-\alpha) F / 2 \\
f_{4}=\alpha F
\end{gathered}
$$

where $F$ is the net load on the edge. Notice for $\alpha=0$ that the load is divided equally between the vertex nodes. For $\alpha=1$, the load is transferred entirely to the mid-edge node. For $\alpha=1 / 5$, the load on a vertex node is twice that on the mid-edge node. Similar expressions hold for the other two edges.

The eight-node tetrahedron is defined to have four vertex nodes and four mid-face nodes as shown in Figure 1b. The nodal weights for the element are chosen as

$$
\left(\hat{w}_{1}, \ldots, \hat{w}_{8}\right)=(1-\alpha, 1-\alpha, 1-\alpha, 1-\alpha, 9 \alpha, 9 \alpha, 9 \alpha, 9 \alpha)
$$

When $\alpha=1 / 10$, the weighting for each node is identical. Consider a surface traction of constant value applied to the face shared by nodes $1,2,3$ and 8 . The equivalent nodal forces are given by

$$
\begin{gathered}
f_{1}=(1-\alpha) F / 3, \quad f_{2}=(1-\alpha) F / 3, \quad f_{3}=(1-\alpha) F / 3 \\
f_{8}=\alpha F
\end{gathered}
$$

where $F$ is the net load on the face. Again, for $\alpha=0$ the load is divided equally between the vertex nodes. For $\alpha=1$, the load is transferred entirely to the mid-face node. For $\alpha=1 / 10$, the load on a vertex node is three times that on the mid-face node. Similar expressions hold for the other three faces.

The ten-node tetrahedron is defined to have four vertex nodes and six mid-edge nodes as shown in Figure 1c. The nodal weights for the element are chosen as

$$
\left(\hat{w}_{1}, \ldots, \hat{w}_{10}\right)=(1-\alpha, 1-\alpha, 1-\alpha, 1-\alpha, 2 \alpha, 2 \alpha, 2 \alpha, 2 \alpha, 2 \alpha, 2 \alpha)
$$

When $\alpha=1 / 3$, the weighting is uniform. Consider a surface traction of constant value applied to the face shared by nodes $1,2,3,5,6$ and 7 . The equivalent nodal forces are given by

$$
\begin{gathered}
f_{1}=(1-\alpha) F / 3, \quad f_{2}=(1-\alpha) F / 3, \quad f_{3}=(1-\alpha) F / 3 \\
f_{5}=\alpha F / 3, \quad f_{6}=\alpha F / 3, \quad f_{7}=\alpha F / 3
\end{gathered}
$$


Notice for $\alpha=0$ that the load is divided equally between the vertex nodes. For $\alpha=1$, the load is shared equally by the mid-edge nodes. For $\alpha \doteq 1 / 3$; the load on a vertex node is twice that on a mid-edge node. Similar expressions hold for the other three faces.

Remark: The case of $\alpha=1$ corresponds to mean quadrature of a standard ten-node tetrahedron with quadratic interpolation of the displacements. The implication for the standard ten-node tetrahedron is that the mid-edge nodes are solely responsible for communicating the mean behavior and the vertex nodes are related to non-constant strain behavior exclusively.

Patch tests of types A through C (see Ref. [2]) were performed for meshes of six-node triangles, eight-node tetrahedra, and ten-node tetrahedra. In all cases, the patch tests were passed provided the mid-edge and mid-face nodes were centered (see Appendix). Satisfaction of the patch tests guarantees convergence as element sizes are reduced.

\section{Example Problems}

Example problems in 2D and 3D linear elasticity are presented in this section. The first example shows that elements generated using the present approach do not suffer from volumetric locking. The second example examines the variation of element eigenvalues with the weighting parameter $\alpha$.

All the examples presented here assume small deformations of a linear, elastic, isotropic material. As such, it is convenient to assemble the element stiffness matrices into the system stiffness matrix. With reference to Eq. (12), an element stiffness matrix $K_{e}$ for $3 \mathrm{D}$ problems is given by

$$
K_{e}=V B^{T} H B
$$

where

$$
\begin{gathered}
H=\left[\begin{array}{cccccc}
2 G+\lambda & \lambda & \lambda & 0 & 0 & 0 \\
\lambda & 2 G+\lambda & \lambda & 0 & 0 & 0 \\
\lambda & \lambda & 2 G+\lambda & 0 & 0 & 0 \\
0 & 0 & 0 & G & 0 & 0 \\
0 & 0 & 0 & 0 & G & 0 \\
0 & 0 & 0 & 0 & 0 & G
\end{array}\right] \\
G=\frac{E}{2(1+\nu)} \\
\lambda=\frac{E \nu}{(1+\nu)(1-2 \nu)}
\end{gathered}
$$

and $E$ is Young's modulus and $\nu$ is Poisson's ratio of the material. For 2D plane strain, the 
matrix $H$ is given by

$$
H=\left[\begin{array}{ccc}
2 G+\lambda & \lambda & 0 \\
\lambda & 2 G+\lambda & 0 \\
0 & 0 & G
\end{array}\right]
$$

and for 2D plane stress,

$$
H=\frac{E}{1-\nu^{2}}\left[\begin{array}{ccc}
1 & \nu & 0 \\
\nu & 1 & 0 \\
0 & 0 & (1-\nu) / 2
\end{array}\right]
$$

For 2D problems, the matrix $B$ in Eq. (34) consists of the first three rows of $\left(\Phi^{T} W \Phi\right)^{-1} \Phi^{T} W$.

Example 3.1: The first example makes use of the 2D and 3D meshes shown in Figure 2. The tetrahedral meshes each consist of 320 elements (five element decomposition of each cubic block).

For the 2D analysis, nodes on the boundaries of the square mesh of triangular elements are subjected to the prescribed displacements

$$
\begin{aligned}
& u(x, y, z)=a\left(y^{2}-x^{2}+2 x y\right) \\
& v(x, y, z)=a\left(x^{2}-y^{2}+2 y x\right)
\end{aligned}
$$

The plane strain assumption with unit element thickness is used.

For the 3D analysis, nodal displacements on the boundaries of the cubical mesh of tetrahedral elements are specified as

$$
\begin{array}{r}
u(x, y, z)=a\left(y^{2}+z^{2}-2 x^{2}+2 x y+2 x z+5 y z\right) \\
v(x, y, z)=a\left(z^{2}+x^{2}-2 y^{2}+2 y z+2 y x+5 z x\right) \\
w(x, y, z)=a\left(x^{2}+y^{2}-2 z^{2}+2 z x+2 z y+5 x y\right)
\end{array}
$$

The elasticity solutions to the 2D and 3D boundary value problems are given by Eqs. (40-44) as well. The deviatoric strain energies for the two problems are given by

$$
\begin{aligned}
& E_{\text {dev }}^{2 D}=32 G a^{2}(10)^{4} / 3 \\
& E_{\text {dev }}^{3 D}=144 G a^{2}(10)^{5}
\end{aligned}
$$

One can confirm that the elasticity solutions have no volumetric strain. That is,

$$
\frac{\partial u}{\partial x}+\frac{\partial v}{\partial y}+\frac{\partial w}{\partial z}=0
$$

Consequently, the exact value of the volumetric strain energy $E_{v o l}$ is zero. 
Calculated values of the volumetric and deviatoric strain energies for the $2 \mathrm{D}$ problem are shown in Table 1. Results are presented for meshes of three-node and six-node triangles for a material with $E=10^{7}$. Three different values of the hourglass stiffness parameter $\epsilon$ were considered and $G_{h}$ was set equal to $G$. The weighting parameter $\alpha$ was set equal to $1 / 5$. This value of $\alpha$ results in equal weighting of the vertex and mid-edge nodes (see Eq. 25).

It is evident in Table 1 that the constant strain three-node triangular element performs poorly for values of $\nu$ near 0.5 . Values of $E_{\text {vol }}$ are significantly lower for the six-node triangular mesh for all the values of $\nu$ and $\epsilon$ shown. In contrast to the three-node triangular mesh, the volumetric strain energy of the six-node triangular mesh decreases as Poisson's ratio is increased.

A plot of $E_{\text {dev }}$ and $E_{v o l}$ versus $\alpha$ for the same material with $\nu=0.499$ and $\epsilon=0.5$ are shown in Figure 3. It is noted that setting $\alpha=0$ (zero weight for mid-edge nodes) leads to results which are identical to those for the three-node triangular mesh. Very small values of volumetric strain energy are obtained for values of $\alpha$ ranging from 0.2 to 1 .

Calculated values of $E_{v o l}$ and $E_{d e v}$ for the 3D problem are shown in Table 2. Results are presented for meshes of four-node, eight-node, and ten-node tetrahedra. Results for the eight and ten-node tetrahedral meshes were obtained by setting all of the nodal weights equal. This nodal weighting corresponds to $\alpha=1 / 10$ for the eight-node element and $\alpha=1 / 3$ for the ten-node element (see Eqs. 28 and 31). Values of $\epsilon$ equal to 0.05 and 0.1 were used for the eight-node and ten-node elements, respectively. In addition, $G_{h}$ was set equal to $G$.

It is evident in Table 2 that the constant strain four-node tetrahedral element performs poorly for values of $\nu$ near 0.5 . Values of $E_{v o l}$ are consistently lower for the eight and tennode tetrahedral meshes. The eight-node element performs much better than the ten-node element for values of $\nu$ near $1 / 2$. Nevertheless, the performance of the ten-node element is significantly better than that of the four-node element.

Plots of $E_{d e v}$ and $E_{v o l}$ versus $\alpha$ for $\nu=0.499$ are shown in Figures 4 and 5. Setting $\alpha=0$ for the eight and ten-node tetrahedral elements leads to results which are identical to those for the four-node element, since this limiting case for the least squares fitting results in using the vertex nodes only.

Plots of the energy norm (see Ref. 2) for the eight-node tetrahedron with $\alpha=1 / 10$ and a uniform strain eight-node hexahedron are shown in Figure 6 for $\nu=0.499$. The hourglass control used for the two element types was specified by $\epsilon=0.05$ and $G_{h}=G$. The convergence rate and accuracy of the eight-node tetrahedron compares favorably with the uniform strain hexahedron. The slopes near unity of the two lines in the figure are consistent with the convergence rate of linear elements.

Example 3.2: The second example examines the variation of element eigenvalues with the weighting parameter $\alpha$. To simplify the analysis, we consider element geometries of an 
equilateral triangle and tetrahedron with unit edge length. Coordinates of the tetrahedron vertices are given by $(0,0,0),(1,0,0),(1 / 2, \sqrt{3} / 2,0)$, and $(1 / 2, \sqrt{3} / 6, \sqrt{6} / 3)$. The geometry of the equilateral triangle is described by the first three vertices. The hourglass stiffness parameter $\epsilon$ is set equal to zero for the results presented.

The six-node triangular element has three rigid body modes, six zero-energy hourglass modes, and three modes with nonzero eigenvalues. Of the three nonzero eigenvalues, two are identical and are associated with shear deformation. The third eigenvalue is associated with the state of strain $\epsilon_{x}=\epsilon_{y}$ and $\gamma_{x y}=0$. For plane strain, one can verify that the eigenvalues are given by

$$
\begin{aligned}
& \lambda_{1}=4 G\left(1-2 \alpha+5 \alpha^{2}\right) V \\
& \lambda_{2}=4(G+\lambda)\left(1-2 \alpha+5 \alpha^{2}\right) V
\end{aligned}
$$

and for plane stress,

$$
\begin{aligned}
& \lambda_{1}=4 G\left(1-2 \alpha+5 \alpha^{2}\right) V \\
& \lambda_{2}=\frac{2 E}{1-\nu}\left(1-2 \alpha+5 \alpha^{2}\right) V
\end{aligned}
$$

Notice that the eigenvalues are a quadratic function of $\alpha$. The smallest eigenvalues are obtained for $\alpha=1 / 5$. This value of $\alpha$ corresponds to equal weighting of vertex and midedge nodes. As expected, the eigenvalues for $\alpha=0$ are identical to those of a constant strain three-node triangle.

The eight-node tetrahedral element has six rigid body modes, twelve zero-energy hourglass modes, and six modes with nonzero eigenvalues. Of the six nonzero eigenvalues, five are identical and are associated with shear deformation. The sixth eigenvalue is associated with a state of hydrostatic strain. Expressions for these eigenvalues are given by

$$
\begin{aligned}
& \lambda_{1}=4 G\left(1-2 \alpha+10 \alpha^{2}\right) V \\
& \lambda_{2}=\frac{2 E}{1-2 \nu}\left(1-2 \alpha+10 \alpha^{2}\right) V
\end{aligned}
$$

As with the six-node triangular element, the eigenvalues are a quadratic function of $\alpha$. The eigenvalues are minimized for $\alpha=1 / 10$. This value of $\alpha$ corresponds to equal weighting of vertex and mid-face nodes. Again, the eigenvalues for $\alpha=0$ are identical to those of a constant strain four-node tetrahedron.

The ten-node tetrahedral element has six rigid body modes, eighteen zero-energy hourglass modes, and six modes with nonzero eigenvalues. Of the six nonzero eigenvalues, five 
are identical and are associated with shear deformation. The sixth eigenvalue is associated with a state of hydrostatic strain. Expressions for these eigenvalues are given by

$$
\begin{aligned}
& \lambda_{1}=4 G\left(1-2 \alpha+3 \alpha^{2}\right) V \\
& \lambda_{2}=\frac{2 E}{1-2 \nu}\left(1-2 \alpha+3 \alpha^{2}\right) V
\end{aligned}
$$

Notice that the eigenvalues are minimized for $\alpha=1 / 3$. This value of $\alpha$ corresponds to equal weights for the vertex and mid-edge nodes. As with the eight-node element, the eigenvalues for $\alpha=0$ are identical to those of a constant strain four-node tetrahedron.

\section{Conclusions}

A new method for deriving uniform strain triangular and tetrahedral finite elements is presented. The method is computationally efficient and avoids the volumetric locking problems common to fully-integrated lower-order elements. The weighted least squares formulation permits surface loads to be distributed in a continuously varying manner between vertex, mid-edge and mid-face nodes. This flexibility in the element formulation may prove useful for applications involving contact where a uniform normal stiffness is desirable. Elements generated using the method pass a suite of patch tests provided the mid-edge and mid-face nodes are centered.

An alternative formulation based on mean quadrature is also presented. Such a formulation is identical to the least squares approach provided the mid-edge and mid-face nodes are centered. The alternative formulation shares all the computational advantages of the least squares approach and can use the same method of hourglass control. Moreover, satisfaction of patch tests does not require centered placement of the mid-edge or mid-face nodes. Work is currently underway to evaluate the performance of the elements for applications involving nonlinear (large) deformations.

\section{Appendix} define

A closed-form expression for $\left(\Phi^{T} W \Phi\right)^{-1} \Phi^{T} W$ is presented in this section. To begin,

$$
\hat{x}_{i}=\hat{w}_{i} x_{i}, \quad \hat{y}_{i}=\hat{w}_{i} y_{i}, \quad \hat{z}_{i}=\hat{w}_{i} z_{i}
$$

where there is no summation on $i$ in Eq. (56). After a significant amount of algebra, one 
arrives at the following expression:

$$
\left(\Phi^{T} W \Phi\right)^{-1} \Phi^{T} W=\left[\begin{array}{cccccccccc}
a_{11} & 0 & 0 & a_{12} & 0 & 0 & \ldots & a_{1 n} & 0 & 0 \\
0 & a_{21} & 0 & 0 & a_{22} & 0 & \ldots & 0 & a_{2 n} & 0 \\
0 & 0 & a_{31} & 0 & 0 & a_{32} & \ldots & 0 & 0 & a_{3 n} \\
a_{21} & a_{11} & 0 & a_{22} & a_{12} & 0 & \ldots & a_{2 n} & a_{1 n} & 0 \\
0 & a_{31} & a_{21} & 0 & a_{32} & a_{22} & \ldots & 0 & a_{3 n} & a_{2 n} \\
a_{31} & 0 & a_{11} & a_{32} & 0 & a_{12} & \ldots & a_{3 n} & 0 & a_{1 n} \\
a_{01} & 0 & 0 & a_{02} & 0 & 0 & \ldots & a_{0 n} & 0 & 0 \\
0 & a_{01} & 0 & 0 & a_{02} & 0 & \ldots & 0 & a_{0 n} & 0 \\
0 & 0 & a_{01} & 0 & 0 & a_{02} & \ldots & 0 & 0 & a_{03} \\
0 & -a_{11} & 0 & 0 & -a_{12} & 0 & \ldots & 0 & -a_{1 n} & 0 \\
0 & 0 & -a_{21} & 0 & 0 & -a_{22} & \ldots & 0 & 0 & -a_{2 n} \\
-a_{31} & 0 & 0 & -a_{32} & 0 & 0 & \ldots & -a_{3 n} & 0 & 0
\end{array}\right]
$$

where

$$
\begin{gathered}
a_{0 i}=\hat{w}_{i} / \sum_{i=1}^{n} \hat{w}_{i} \\
a_{1 i}=c_{1} \hat{x}_{i}+c_{4} \hat{y}_{i}+c_{6} \hat{z}_{i} \\
a_{2 i}=c_{2} \hat{y}_{i}+c_{5} \hat{z}_{i}+c_{4} \hat{x}_{i} \\
a_{3 i}=c_{3} \hat{z}_{i}+c_{6} \hat{x}_{i}+c_{5} \hat{y}_{i} \\
c_{0}=s_{x x} s_{y y} s_{z z}+2 s_{x y} s_{y z} s_{z x}-s_{x x} s_{y z}^{2}-s_{y y} s_{z x}^{2}-s_{z z} s_{x y}^{2} \\
c_{1}=\left(s_{y y} s_{z z}-s_{y z}^{2}\right) / c_{0}, \quad c_{4}=\left(s_{y z} s_{z x}-s_{x y} s_{z z}\right) / c_{0} \\
c_{2}=\left(s_{z z} s_{x x}-s_{z x}^{2}\right) / c_{0}, \quad c_{5}=\left(s_{z x} s_{x y}-s_{y z} s_{x x}\right) / c_{0} \\
c_{3}=\left(s_{x x} s_{y y}-s_{x y}^{2}\right) / c_{0}, \quad c_{6}=\left(s_{x y} s_{y z}-s_{z x} s_{y y}\right) / c_{0}
\end{gathered}
$$

and

$$
\begin{aligned}
& s_{x x}=\sum_{i=1}^{n} x_{i} \hat{x}_{i}, \quad s_{y y}=\sum_{i=1}^{n} y_{i} \hat{y}_{i}, \quad s_{z z}=\sum_{i=1}^{n} z_{i} \hat{z}_{i} \\
& s_{x y}=\sum_{i=1}^{n} x_{i} \hat{y}_{i}, \quad s_{y z}=\sum_{i=1}^{n} y_{i} \hat{z}_{i}, \quad s_{z x}=\sum_{i=1}^{n} z_{i} \hat{x}_{i}
\end{aligned}
$$

For 2D problems, the matrix $\left(\Phi^{T} W \Phi\right)^{-1} \Phi^{T} W$ is obtained by deleting every third column and rows $3,5,6,9,11$ and 12 of the matrix on the right hand side of Eq. (57). In addition, one sets $s_{z z}=1, s_{y z}=0$ and $s_{z x}=0$. 
An alternative formulation based on mean quadrature of a six-node triangle, eight-node tetrahedron, and ten-node tetrahedron is presented here. The method combines ideas from Section 2 and References [1] and [6] to obtain a family of conforming elements. The conditions under which the least squares formulation is equivalent to the alternative formulation are also presented. The eight-node tetrahedral element developed in this section with $\alpha=1 / 3$ is identical to an element developed previously in Reference 6.

To begin, let

$$
\begin{aligned}
A_{i j k}= & {\left[x_{i}\left(y_{j}-y_{k}\right)+x_{j}\left(y_{k}-y_{i}\right)+x_{k}\left(y_{i}-y_{j}\right)\right] / 2 } \\
V_{i j k l}= & {\left[\left(x_{j}-x_{i}\right)\left(y_{k} z_{l}-y_{l} z_{k}\right)+\left(x_{i}-x_{k}\right)\left(y_{j} z_{l}-y_{l} z_{j}\right)+\left(x_{l}-x_{i}\right)\left(y_{j} z_{k}-y_{k} z_{j}\right)+\right.} \\
& \left.\left(x_{k}-x_{j}\right)\left(y_{i} z_{l}-y_{l} z_{i}\right)+\left(x_{j}-x_{l}\right)\left(y_{i} z_{k}-y_{k} z_{i}\right)+\left(x_{l}-x_{k}\right)\left(y_{i} z_{j}-y_{j} z_{i}\right)\right] / 6
\end{aligned}
$$

where $A_{i j k}$ and $V_{i j k l}$ denote the area and volume of a triangle and tetrahedron with vertices $(i, j, k)$ and $(i, j, k, l)$, respectively. Consider a hexagon (six-node triangle), a polyhedron with eight vertices (eight-node tetrahedron), and a polyhedron with ten vertices (ten-node tetrahedron) with volumes given by

$$
\begin{aligned}
A_{6}= & A_{123}+2 \alpha\left(A_{253}+A_{361}+A_{142}\right) \\
V_{8}= & V_{1234}+3 \alpha\left(V_{2345}+V_{1436}+V_{1247}+V_{1328}\right) \\
V_{10}= & (1-4 \alpha / 3) V_{1234}+4 \alpha\left(V_{1578}+V_{5269}+V_{63710}+V_{89104}+V_{895 c}+\right. \\
& \left.V_{9106 c}+V_{7108 c}+V_{567 c}+V_{578 c}+V_{596 c}+V_{6107 c}+V_{8109 c}\right) / 3
\end{aligned}
$$

where

$$
\left(x_{c}, y_{c}, z_{c}\right)=\left[\left(x_{5}, y_{5}, z_{5}\right)+\left(x_{6}, y_{6}, z_{6}\right)+\ldots+\left(x_{10}, y_{10}, z_{10}\right)\right] / 6
$$

In the present development, nodes 4,5 and 6 of the hexagon remain associated with edges 12,23 , and 31 of triangle 123 (see Figure 1a), but are no longer constrained to the midedge positions. Likewise, nodes $5,6,7$ and 8 of the polyhedron with eight vertices remain associated with faces 234, 143, 124, and 132 of tetrahedron 1234 (see Figure 1b), but are no longer constrained to the mid-face positions. Similar flexibility is afforded to nodes 5 through 10 of the polyhedron with ten vertices.

One can show that a hexagon with edges $1 \hat{4}, \hat{4} 2,2 \hat{5}, \hat{5} 3,3 \hat{6}$, and $\hat{6} 1$ has area $A_{6}$ where

$$
\begin{aligned}
& \left(\hat{x}_{4}, \hat{y}_{4}\right)=2 \alpha\left(x_{4}, y_{4}\right)+(1-2 \alpha)\left(x_{1}+x_{2}, y_{1}+y_{2}\right) / 2 \\
& \left(\hat{x}_{5}, \hat{y}_{5}\right)=2 \alpha\left(x_{5}, y_{5}\right)+(1-2 \alpha)\left(x_{2}+x_{3}, y_{2}+y_{3}\right) / 2 \\
& \left(\hat{x}_{6}, \hat{y}_{6}\right)=2 \alpha\left(x_{6}, y_{6}\right)+(1-2 \alpha)\left(x_{3}+x_{1}, y_{3}+y_{1}\right) / 2
\end{aligned}
$$


Likewise, a polyhedron with triangular faces $23 \hat{5}, 34 \hat{5}, 42 \hat{5}, 31 \hat{6}, 14 \hat{6}, 43 \hat{6}, 12 \hat{7}, 24 \hat{7}, 41 \hat{7}, 21 \hat{8}$, $13 \hat{8}$, and $32 \hat{8}$ has volume $V_{8}$ where

$$
\begin{aligned}
& \left(\hat{x}_{5}, \hat{y}_{5}, \hat{z}_{5}\right)=3 \alpha\left(x_{5}, y_{5}, z_{5}\right)+(1-3 \alpha)\left(x_{2}+x_{3}+x_{4}, y_{2}+y_{3}+y_{4}, z_{2}+z_{3}+z_{4}\right) / 3 \\
& \left(\hat{x}_{6}, \hat{y}_{6}, \hat{z}_{6}\right)=3 \alpha\left(x_{6}, y_{6}, z_{6}\right)+(1-3 \alpha)\left(x_{1}+x_{4}+x_{3}, y_{1}+y_{4}+y_{3}, z_{1}+z_{4}+z_{3}\right) / 3 \\
& \left(\hat{x}_{7}, \hat{y}_{7}, \hat{z}_{7}\right)=3 \alpha\left(x_{7}, y_{7}, z_{7}\right)+(1-3 \alpha)\left(x_{1}+x_{2}+x_{4}, y_{1}+y_{2}+y_{4}, z_{1}+z_{2}+z_{4}\right) / 3 \\
& \left(\hat{x}_{8}, \hat{y}_{8}, \hat{z}_{8}\right)=3 \alpha\left(x_{8}, y_{8}, z_{8}\right)+(1-3 \alpha)\left(x_{1}+x_{3}+x_{2}, y_{1}+y_{3}+y_{2}, z_{1}+z_{3}+z_{2}\right) / 3
\end{aligned}
$$

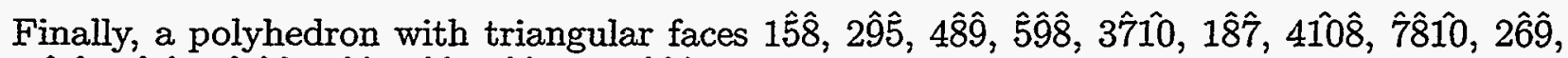
$3 \hat{0} \hat{0} \hat{6}, 4 \hat{9} \hat{10}, 1 \hat{0} \hat{4} \hat{9}, 2 \hat{5} \hat{6}, 1 \hat{7} \hat{5}, 3 \hat{\sigma} \hat{7}$, and $\hat{5} \hat{7} \hat{b}$ has volume $V_{10}$ where

$$
\begin{aligned}
\left(\hat{x}_{5}, \hat{y}_{5}, \hat{z}_{5}\right) & =4 \alpha\left(x_{5}, y_{5}, z_{5}\right) / 3+(1-4 \alpha / 3)\left(x_{1}+x_{2}, y_{1}+y_{2}, z_{1}+z_{2}\right) / 2 \\
\left(\hat{x}_{6}, \hat{y}_{6}, \hat{z}_{6}\right) & =4 \alpha\left(x_{6}, y_{6}, z_{6}\right) / 3+(1-4 \alpha / 3)\left(x_{2}+x_{3}, y_{2}+y_{3}, z_{2}+z_{3}\right) / 2 \\
\left(\hat{x}_{7}, \hat{y}_{7}, \hat{z}_{7}\right) & =4 \alpha\left(x_{7}, y_{7}, z_{7}\right) / 3+(1-4 \alpha / 3)\left(x_{3}+x_{1}, y_{3}+y_{1}, z_{3}+z_{1}\right) / 2 \\
\left(\hat{x}_{8}, \hat{y}_{8}, \hat{z}_{8}\right) & =4 \alpha\left(x_{8}, y_{8}, z_{8}\right) / 3+(1-4 \alpha / 3)\left(x_{1}+x_{4}, y_{1}+y_{4}, z_{1}+z_{4}\right) / 2 \\
\left(\hat{x}_{9}, \hat{y}_{9}, \hat{z}_{9}\right) & =4 \alpha\left(x_{9}, y_{9}, z_{9}\right) / 3+(1-4 \alpha / 3)\left(x_{2}+x_{4}, y_{2}+y_{4}, z_{2}+z_{4}\right) / 2 \\
\left(\hat{x}_{10}, \hat{y}_{10}, \hat{z}_{10}\right) & =4 \alpha\left(x_{10}, y_{10}, z_{10}\right) / 3+(1-4 \alpha / 3)\left(x_{3}+x_{4}, y_{3}+y_{4}, z_{3}+z_{4}\right) / 2
\end{aligned}
$$

It follows from the definition of the hexagon edges and polyhedral faces that meshes of six-node triangles, eight-node tetrahedra or ten-node tetrahedra will be conforming. That is, there is continuity between adjacent element edges and faces for the three element types.

Comparison of the least squares approach (see Eqs. 11-13,57,59-61) and a generalization of the approach presented in Reference [1] (see Eqs. 13,16,58) shows that the two are equivalent provided that

$$
a_{1 i}=\frac{1}{V} \frac{\partial V}{\partial x_{i}} \quad a_{2 i}=\frac{1}{V} \frac{\partial V}{\partial y_{i}} \quad a_{3 i}=\frac{1}{V} \frac{\partial V}{\partial z_{i}}
$$

where $V$ denotes $A_{6}$ for the six-node triangle, $V_{8}$ for the eight-node tetrahedron, and $V_{10}$ for the ten-node tetrahedron. One can show the above equalities hold if the coordinates of the mid-edge and mid-face nodes are given by Eqs. (74-86) with $\alpha$ set equal to zero. That is, the mid-edge and mid-face nodes are geometrically centered.

To compare the two different approaches, one simply uses either Eqs. (59-61) or Eq. (87) to calculate $a_{1 i}, a_{2 i}$ and $a_{3 i}$. The same hourglass control control can be used for either approach. Both approaches pass the patch test if the mid-edge and mid-face nodes are centered, but only the alternative approach presented in this section passes the patch test if the nodes are not centered. For small deformation problems, the difference is not important provided the nodes are centered initially. For large deformation problems we suspect that the alternative formulation may be better suited. 
As with the least squares formulation, the alternative formulation can be implemented efficiently. The derivatives in Eq. (87) can be calculated using

$$
\begin{aligned}
\partial V_{i j k l} / \partial x_{i} & =\left[\left(y_{k}-y_{l}\right)\left(z_{j}-z_{l}\right)-\left(y_{j}-y_{l}\right)\left(z_{k}-z_{l}\right)\right] / 6 \\
\partial V_{i j k l} / \partial y_{i} & =\left[\left(z_{k}-z_{l}\right)\left(x_{j}-x_{l}\right)-\left(z_{j}-z_{l}\right)\left(x_{k}-x_{l}\right)\right] / 6 \\
\partial V_{i j k l} / \partial z_{i} & =\left[\left(x_{k}-x_{l}\right)\left(y_{j}-y_{l}\right)-\left(x_{j}-x_{l}\right)\left(y_{k}-y_{l}\right)\right] / 6
\end{aligned}
$$

In addition, the alternative formulation allows one to ignore specified mid-edge or mid-face nodes. For example, a seven-node tetrahedral element without mid-face node 8 is obtained simply by neglecting the volume $V_{1328}$ in Eq. (71). The least squares formulation can also be modified to ignore certain nodes, but the approach is not as straightforward. The midedge nodes of the six-node triangle and mid-face nodes of the eight-node tetrahedron can be constrained to possess only a normal degree of freedom by simple modifications of the expressions for area and volume in Eqs. (68-69).

Finally, the equivalent nodal loads given in Eqs. (26-27,29-30,32-33) can also be determined by calculating the virtual work done by a uniform distributed force on the edges or faces of the triangular and tetrahedral elements. By making use of Eqs. (74-86), one arrives at the same expressions for the equivalent loads provided the mid-edge and mid-face nodes are centered. 


\section{References}

1. D. P. Flanagan and T. Belytschko, 'A Uniform Strain Hexahedron and Quadrilateral with Orthogonal Hourglass Control', International Journal for Numerical Methods in Engineering, 17, 679-706 (1981).

2. O. C. Zienkiewicz and R. L. Taylor, The Finite Element Method, Vol. 1, 4th Ed., McGraw-Hill, New York, New York, 1989.

3. J. C. Simo and T. J. R. Hughes, 'On the Variational Foundations of Assumed Strain Methods', Journal of Applied Mechanics, 53, 51-54 (1986).

4. T. Belytschko, Y. Krongauz, D. Organ, M. Fleming and P. Krysl, 'Meshless Methods: An Overview and Recent Developments', Computer Methods in Applied Mechanics and Engineering, 139, 3-47 (1996).

5. G. H. Golub and C. F. Van Loan, Matrix Computations, 2nd Ed., John Hopkins, Baltimore, Maryland, 1989.

6. S. W. Key, M. W. Heinstein, C. M. Stone, F. J. Mello, M. L. Blanford and K. G. Budge, 'A Suitable Low-Order, 8-Node Tetrahedral Finite Element for Solids', Sandia National Laboratories Report, Albuquerque, New Mexico (1998). 
Table 1: Strain energies for Example 3.1 (2D analysis, $a=4 \times 10^{-6}$ ).

\begin{tabular}{|c|c|c|c|c|c|c|c|c|c|}
\hline$\nu$ & \multicolumn{7}{|c|}{ three-node } & \multicolumn{7}{|c|}{ six-node } & exact \\
\hline & & & \multicolumn{2}{|c|}{$\epsilon=0.1$} & \multicolumn{2}{|c|}{$\epsilon=0.5$} & \multicolumn{2}{|c|}{$\epsilon=1$} & \\
\hline & $E_{\text {dev }}$ & $E_{\text {vol }}$ & $E_{\text {dev }}$ & $E_{\text {vol }}$ & $E_{\text {dev }}$ & $E_{\text {vol }}$ & $E_{\text {dev }}$ & $E_{\text {vol }}$ & $E_{\text {dev }}$ \\
\hline 0.0 & 8.52 & 0.020 & 8.27 & $3.8 \mathrm{e}-3$ & 8.45 & $4.9 \mathrm{e}-3$ & 8.49 & $1.0 \mathrm{e}-2$ & 8.533 \\
0.1 & 7.75 & 0.024 & 7.53 & $3.7 \mathrm{e}-3$ & 7.68 & $5.2 \mathrm{e}-3$ & 7.72 & $1.1 \mathrm{e}-2$ & 7.758 \\
0.2 & 7.10 & 0.028 & 6.90 & $3.5 \mathrm{e}-3$ & 7.04 & $5.5 \mathrm{e}-3$ & 7.08 & $1.2 \mathrm{e}-2$ & 7.111 \\
0.3 & 6.56 & 0.036 & 6.38 & $3.0 \mathrm{e}-3$ & 6.49 & $5.6 \mathrm{e}-3$ & 6.53 & $1.3 \mathrm{e}-2$ & 6.564 \\
0.4 & 6.10 & 0.056 & 5.93 & $2.1 \mathrm{e}-3$ & 6.03 & $4.9 \mathrm{e}-3$ & 6.06 & $1.4 \mathrm{e}-2$ & 6.095 \\
0.499 & 5.74 & 4.17 & 5.55 & $3.2 \mathrm{e}-5$ & 5.62 & $1.2 \mathrm{e}-4$ & 5.66 & $6.6 \mathrm{e}-4$ & 5.693 \\
\hline
\end{tabular}

Table 2: Strain energies for Example 3.1 (3D analysis, $a=4 \times 10^{-6}$ ).

\begin{tabular}{|c|c|c|c|c|c|c|c|}
\hline$\nu$ & \multicolumn{2}{|c|}{ four-node } & \multicolumn{2}{c|}{ eight-node } & \multicolumn{2}{c|}{ ten-node } & exact \\
\hline & $E_{\text {dev }}$ & $E_{\text {vol }}$ & $E_{\text {dev }}$ & $E_{\text {vol }}$ & $E_{\text {dev }}$ & $E_{\text {vol }}$ & $E_{\text {dev }}$ \\
\hline 0.0 & 1156 & 4.18 & 1142 & 0.383 & 1144 & 0.116 & 1152 \\
0.1 & 1051 & 5.17 & 1038 & 0.366 & 1040 & 0.133 & 1047 \\
0.2 & 963 & 6.81 & 952 & 0.345 & 953 & 0.157 & 960.0 \\
0.3 & 889 & 10.0 & 879 & 0.315 & 880 & 0.197 & 886.2 \\
0.4 & 826 & 19.5 & 816 & 0.256 & 817 & 0.291 & 822.9 \\
0.499 & 773 & 1903 & 762 & 0.007 & 763 & 18.5 & 768.5 \\
\hline
\end{tabular}




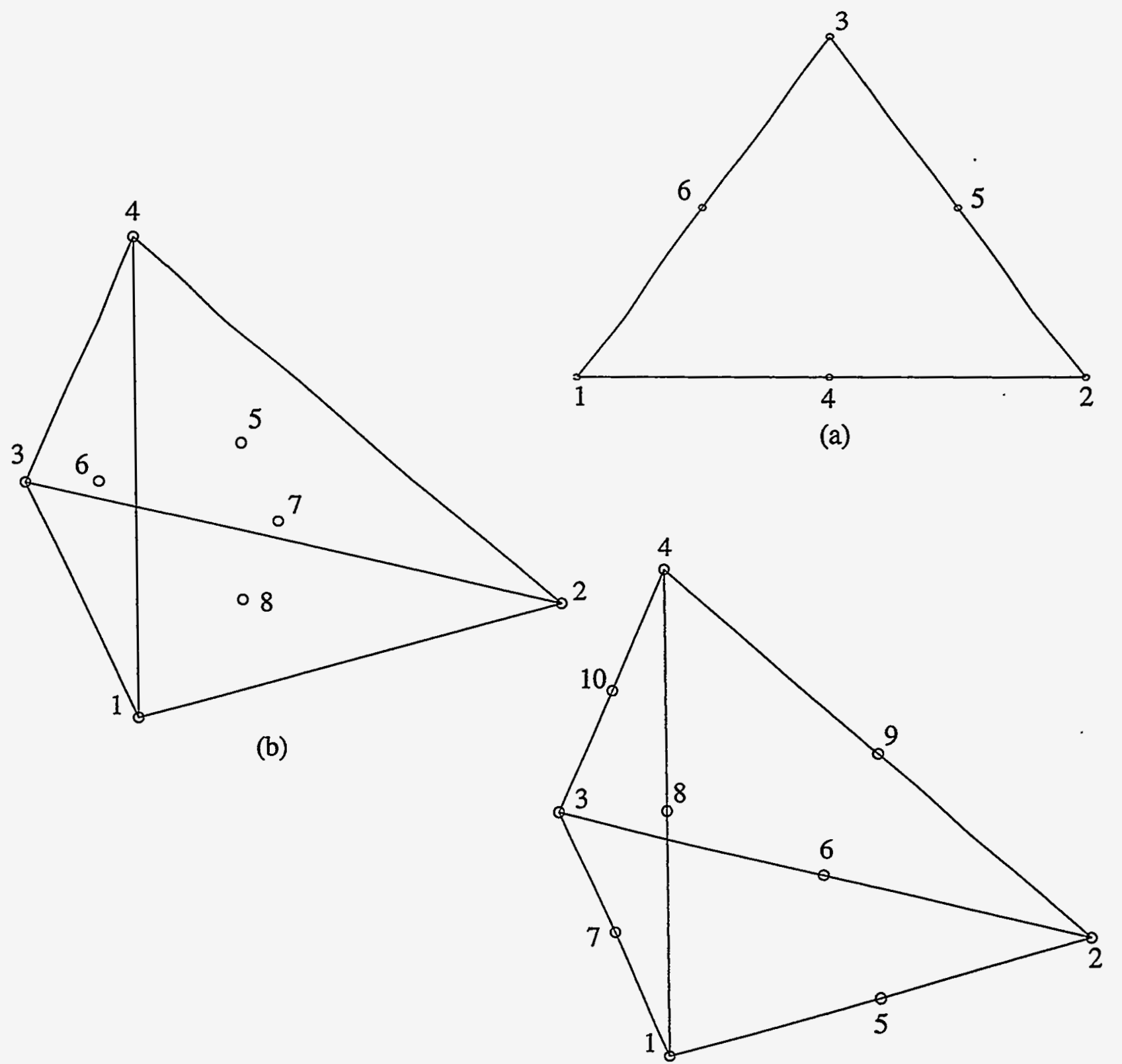

(c)

Figure 1: Element geometries for (a) six-node triangle, (b) eight-node tetrahedron, and (c) ten-node tetrahedron. 

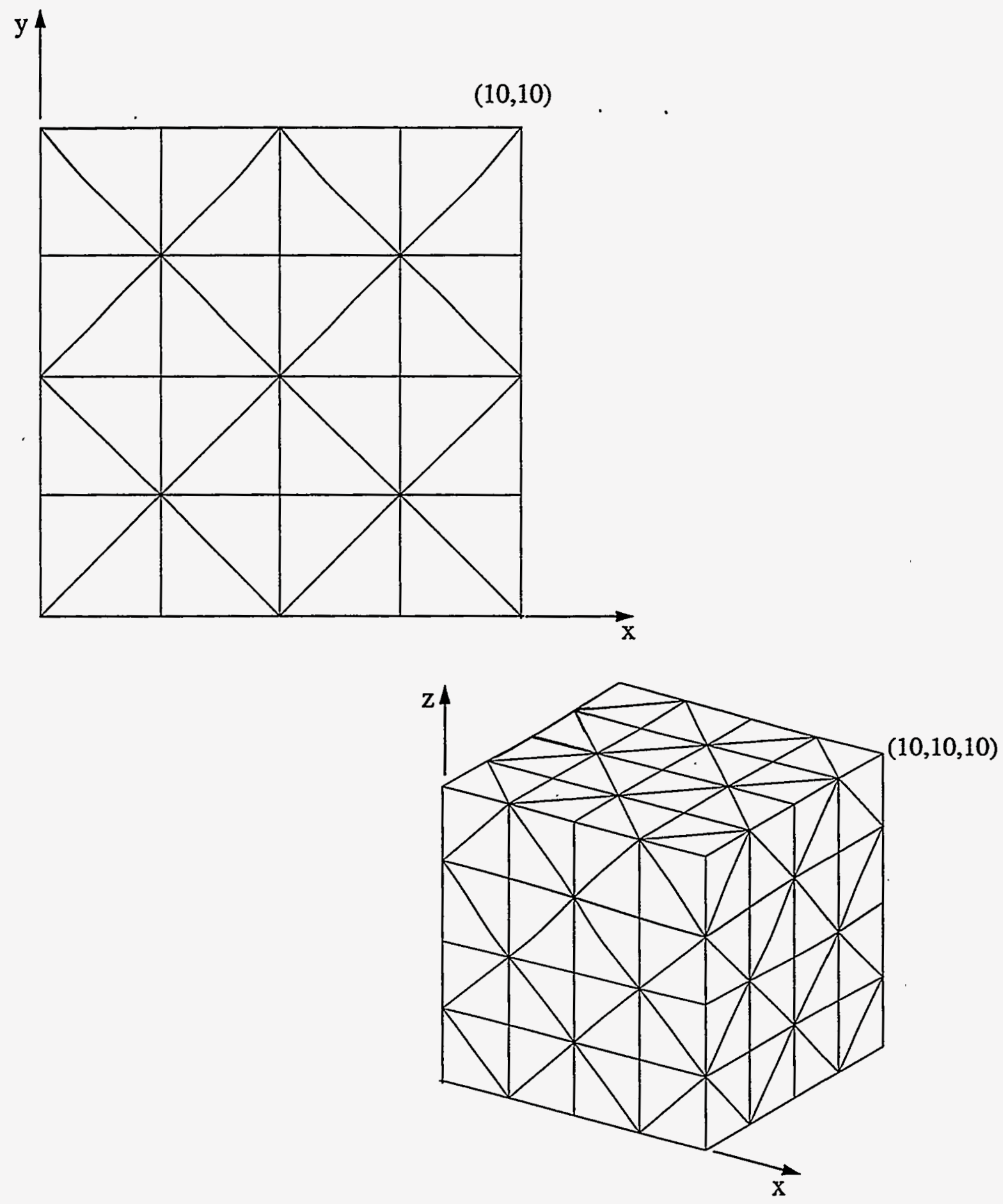

Figure 2: Triangular and tetrahedral meshes used in Example 3.1. 


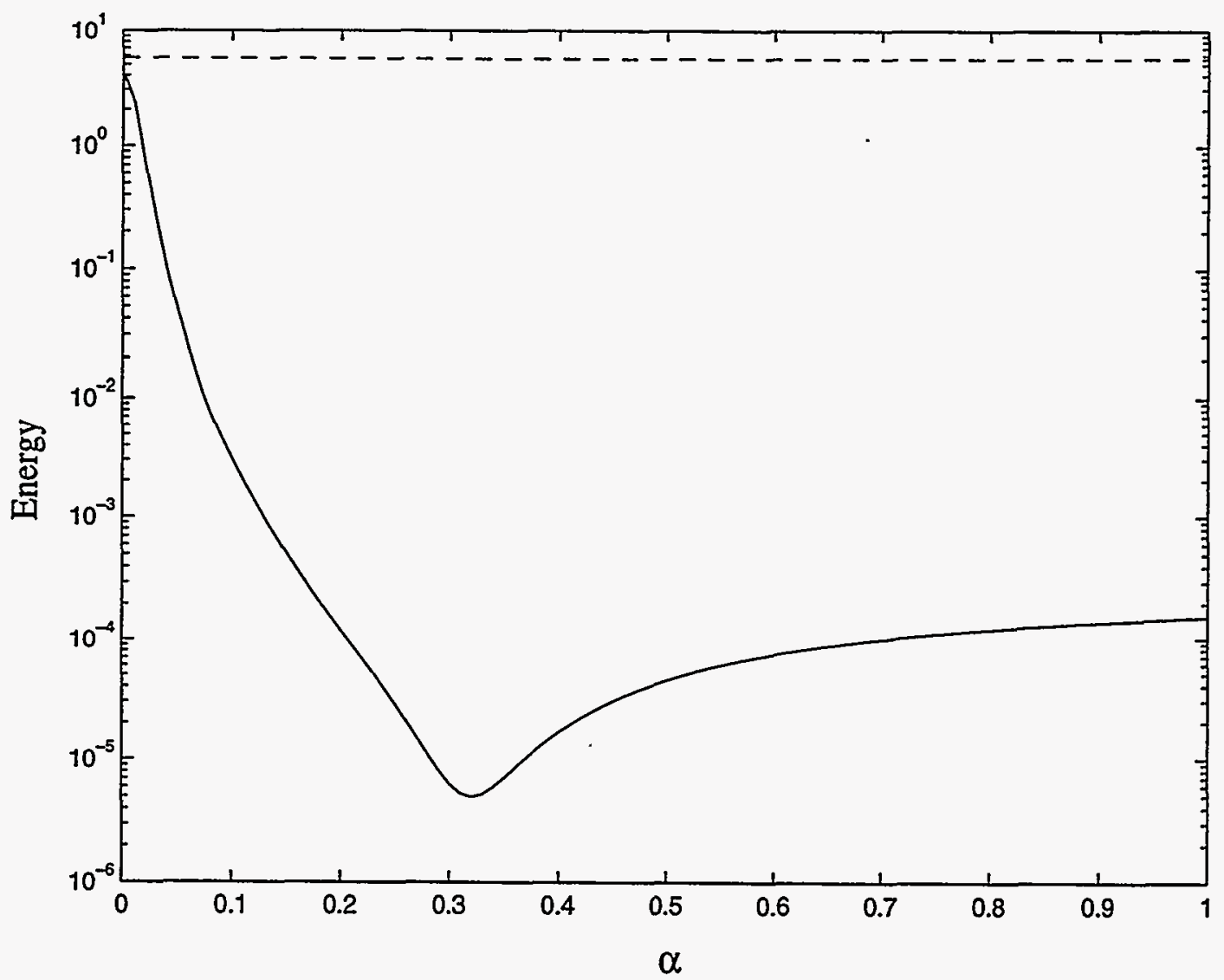

Figure 3: Volumetric (solid line) and deviatoric (dashed line) strain energies for the sixnode triangular mesh. The ideal result for volumetric strain energy is zero. For values of $\alpha$ around 0.3 , the volumetric strain energy is six orders of magnitude lower than the deviatoric strain energy. 


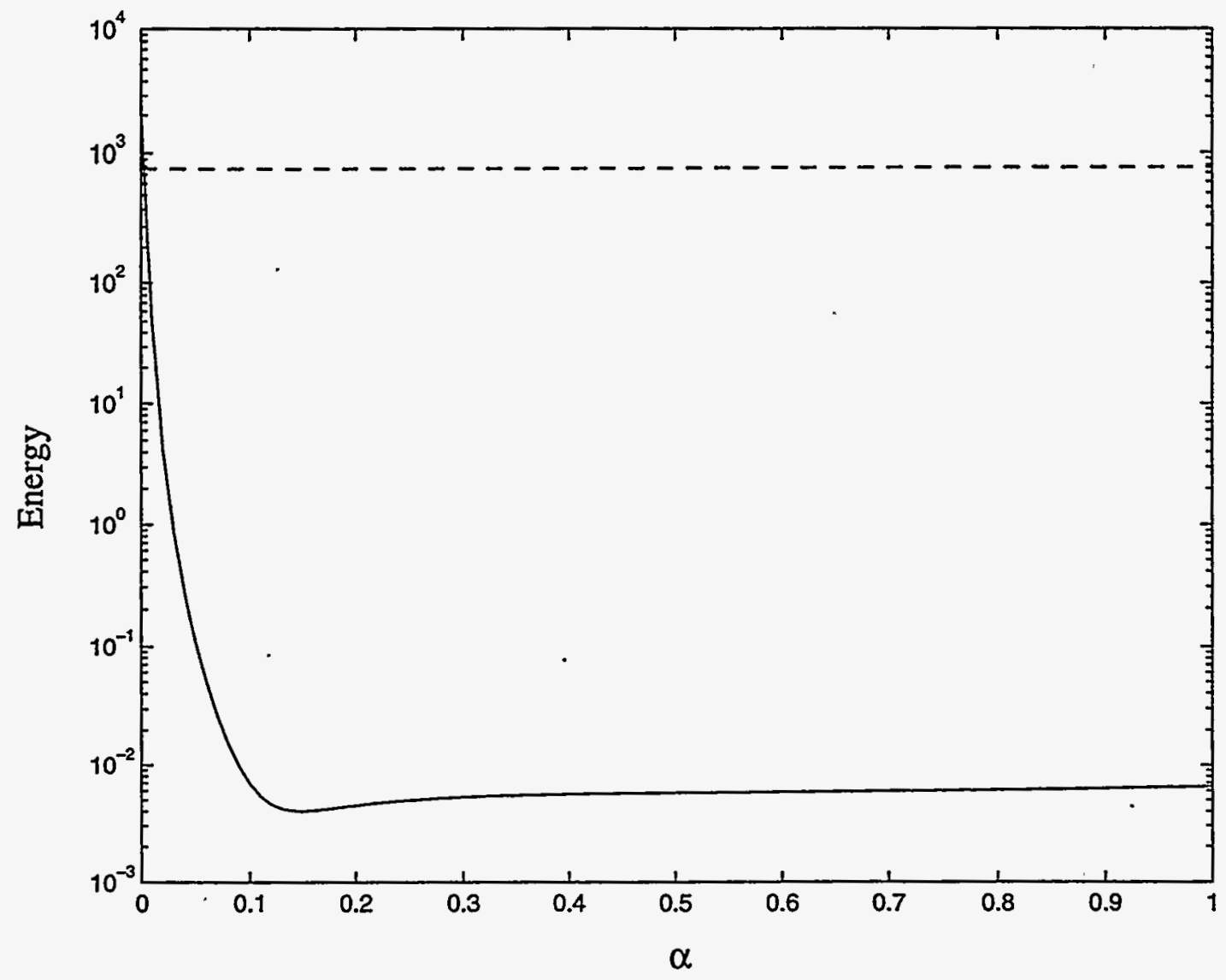

Figure 4: Volumetric (solid line) and deviatoric (dashed line) strain energies for the eight-node tetrahedral mesh. For values of $\alpha$ greater than 0.1 , the volumetric strain energy is five orders of magnitude lower than the deviatoric strain energy. 


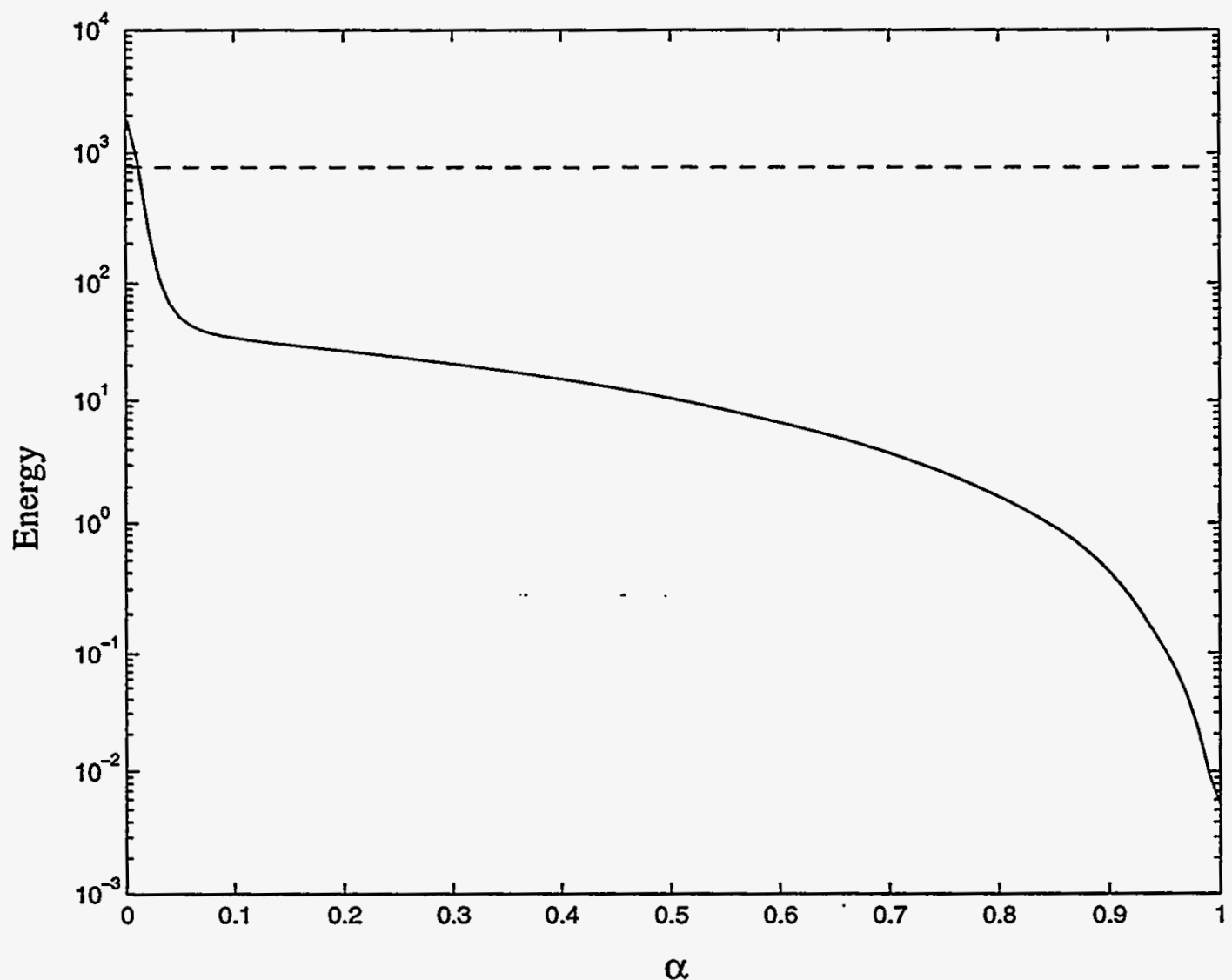

Figure 5: Volumetric (solid line) and deviatoric (dashed line) strain energies for the tennode tetrahedral mesh. The minimum value of volumetric strain energy is for $\alpha$ equal to unity. This weighting corresponds to mean quadrature of a ten-node tetrahedron with quadratic interpolation of the displacements. 


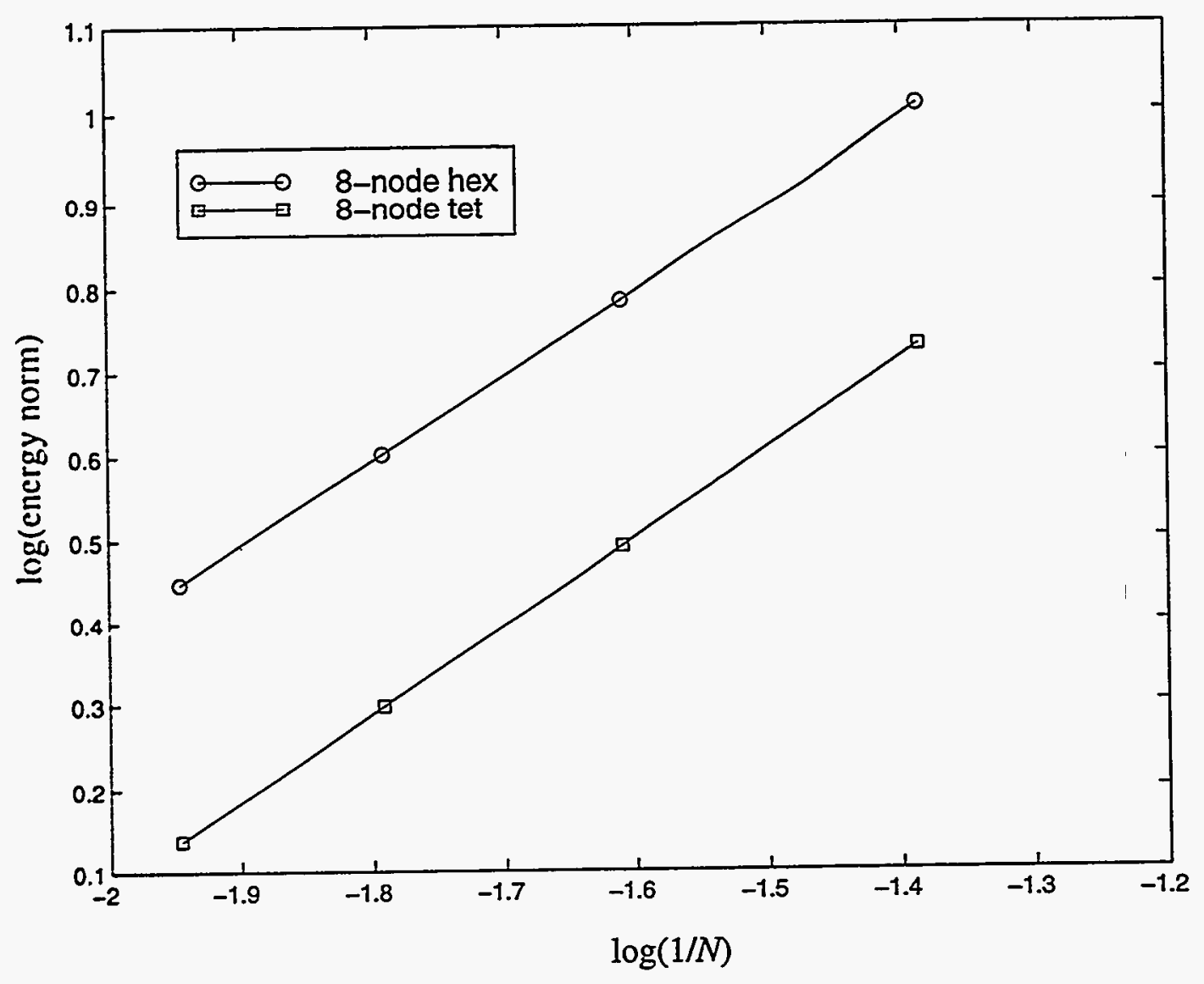

Figure 6: Energy norms of the eight-node tetrahedron and eight-node uniform hexahedron as functions of element divisions per edge $N$. The mesh shown in Figure 2 has $N=4$. The slopes near unity of the two lines are characteristic of linear elements. 


\title{
Enhanced Uniform Strain Triangular and Tetrahedral Finite Elements ${ }^{1}$
}

\author{
C. R. Dohrmann ${ }^{2}$ \\ S. W. $\mathrm{Key}^{3}$
}

\begin{abstract}
A family of enhanced uniform strain triangular and tetrahedral finite elements is presented. Element types considered include a seven-node triangle, nine-node tetrahedron, and eleven-node tetrahedron. Internal nodes are included in the element formulations to permit decompositions of the triangle into three quadrilaterals and the tetrahedra into four hexahedra. Element formulations are based on the standard uniform strain approach for the quadrilateral and hexahedron in conjunction with a set of kinematic constraints. Specification of the constraints allows surface loads to be varied in a continuous manner between vertex and mid-edge nodes for the eleven-node tetrahedron. Comparisons with existing uniform strain elements and elements from a commercial finite element code are included.
\end{abstract}

Key Words. Finite elements, uniform strain, hourglass control, contact.

\footnotetext{
${ }^{1}$ Sandia is a multiprogram laboratory operated by Sandia Corporation, a Lockheed Martin Company, for the United States Department of Energy under Contract DE-AL04-94AL8500.

${ }^{2}$ Structural Dynamics Department, Sandia National Laboratories, MS 0439, Albuquerque, New Mexico 87185-0439, email: crdohrm@sandia.gov, phone: (505) 844-8058, fax: (505) 844-9297.

${ }^{3}$ Engineering and Manufacturing Mechanics Department, Sandia National Laboratories, MS 0443, Albuquerque, New Mexico 87185-0443.
} 


\section{Introduction}

A family of uniform strain elements was recently introduced that includes a six-node triangle, eight-node tetrahedron, and ten-node tetrahedron [1]. Although computationally efficient, each of these element types can only approximate a single uniform state of strain. As a consequence, the elements have several zero energy hourglass modes that must be controlled. The number of hourglass modes for the six-node triangle, eight-node tetrahedron and ten-node tetrahedron is six, twelve and eighteen, respectively.

The purpose of this study is to investigate specific enhancements to improve the accuracy of the uniform strain elements of Ref. 1 . By adding an internal "center" node to each element type, it is possible to geometrically decompose the triangle into three quadrilaterals and the tetrahedra into four hexahedra. Within each of the quadrilateral or hexahedral domains, the element formulations are based on the standard uniform strain approach [2]. 'Element formulations for the nine-node tetrahedron and eleven-node tetrahedron also include a set of kinematic constraints.

The present approach is motivated by the idea that the accuracy of uniform strain elements may be improved by allowing more than a single state of uniform strain. Based on this idea, clear improvements in element accuracy are demonstrated for the seven-node triangle and eleven-node tetrahedron. In addition, allowing multiple states of uniform strain leads to reduced numbers of hourglass modes. The number of hourglass modes for the sevennode triangle, nine-node tetrahedron and eleven-node tetrahedron is two, three and three, respectively.

The enhanced elements can be viewed as the union of uniform strain elements with certain nodes subject to kinematic constraints. Virtual nodes are introduced in the element formulations for the tetrahedra to permit the geometric decompositions described above. Movement of the virtual nodes is constrained to the vertex and mid-edge nodes of the parent elements. For example, the eleven-node tetrahedron is the union of four hexahedra. Virtual mid-face nodes of the tetrahedron serve as vertices of each of the four hexahedra. Motion of these mid-face nodes is constrained to the adjacent mid-edge and vertex nodes.

The idea of decomposing a triangle into three quadrilaterals and a tetrahedron into four hexahedra is not new. In addition, one can directly use these elementary decompositions together with the element formulations for the uniform strain quadrilateral and hexahedron to perform an analysis. That being the case, one may question the advantages of the present approach. For the nine-node and eleven-node tetrahedron, there are fewer numbers of nodes than there are for the union of four hexahedra. In addition, internal nodal displacements of the triangle and two tetrahedra can be solved for in terms of the remaining nodal displacements prior to assembly of the equations for static analysis. Another advantage is that the computations required for hourglass control are reduced in comparison to those for three quadrilaterals or four hexahedra. For example, the hourglass control forces for the elevennode tetrahedron can be calculated all at once rather than by accumulating the forces of four separate hexahedra.

The eleven-node tetrahedron also has a distinct advantage over the standard quadratic ten-node tetrahedron for applications involving contact. For a uniform pressure distribution, 
the ten-node quadratic tetrahedron distributes the load entirely at the mid-edge nodes. With the present formulation, a uniform pressure can be distributed in a continuous manner between vertex and mid-edge nodes. This flexibility in the element formulation may prove useful for applications involving contact where a uniform normal stiffness is desirable.

Details of the approach are provided in the following section. Example problems in 2D and $3 \mathrm{D}$ linear elasticity are given in Section 3 . The example problems include comparisons with the uniform strain elements of Ref. 1 and elements from a commercial finite element code.

\section{Element Formulations}

Element formulations for the seven-node triangle, nine-node tetrahedron and eleven-node tetrahedron are presented in this section. The formulations are based on the uniform strain approach of Reference 2 in conjunction with a set of kinematic constraints. The coordinates and displacements of node $I$ in a Cartesian frame are denoted by $x_{i I}$ and $u_{i I}$, respectively. For $2 \mathrm{D}$ elements. the index $i$ varies from 1 to 2 . For 3D elements, $i$ varies from 1 to 3 .

Elements of the $B$ matrix of the quadrilateral shown in Figure 1a are defined as

$$
B_{i I}^{q u a d}=\frac{\partial A}{\partial x_{i I}}
$$

where $A$ is the area of the quadrilateral. Following the development in Ref. 2, the nodal forces associated with the element stresses are given by

$$
f_{i I}^{q u a d}=\sum_{j=1}^{2} \bar{T}_{i j} B_{j I}^{q u a d}
$$

where $\bar{T}_{\imath \jmath}$ are elements of the Cauchy stress tensor (assumed constant throughout the element).

Elements of the $B$ matrix of the hexahedron shown in Figure $1 \mathrm{~b}$ are defined as

$$
B_{i I}^{h e x}=\frac{\partial V}{\partial x_{i I}}
$$

where $V$ is the volume of the hexahedron. Nodal forces associated with the element stresses are given by

$$
f_{i I}^{h e x}=\sum_{j=1}^{3} \bar{T}_{i j} B_{j I}^{h e x}
$$

Explicit expressions for the elements of the $B$ matrices of the quadrilateral and hexahedron are provided in Ref. 2.

\subsection{Seven-Node Triangle}

A sketch of the enhanced uniform strain seven-node triangle (EUST7) is shown in Figure 2a. The element consists of vertex nodes $1,2,3$, mid-edge nodes 4,5,6 and center node 
7. The triangle is geometrically decomposed into three quadrilaterals specified by the nodal 4-tuples 1476, 2574 and 3675 (see Figure 2b).

Consider a distributed load on edge 12 that varies linearly between nodes 1 and 2 . Assuming node 4 is centered between nodes 1 and 2 , the equivalent nodal forces, obtained using the principle of virtual work, are given by

$$
f_{1}=\left(5 p_{1} / 24+p_{2} / 24\right) L \quad f_{2}=\left(5 p_{2} / 24+p_{1} / 24\right) L \quad f_{4}=\left(p_{1}+p_{2}\right) L / 4
$$

where $p_{1}$ and $p_{2}$ are the values of the distributed load at nodes 1 and 2 and $L$ is the length of edge 12. Similar expressions hold for the other two edges of the element. For $p_{1}=p_{2}=p$, Eq. (5) simplifies to

$$
f_{1}=p L / 4 \quad f_{2}=p L / 4 \quad f_{4}=p L / 2
$$

For purposes of comparison, the equivalent nodal forces for a triangle with quadratic interpolation are given by

$$
f_{1}=p L / 6 \quad f_{2}=p L / 6 \quad f_{4}=2 p L / 3
$$

\subsection{Nine-Node Tetrahedron}

Consider the enhanced uniform strain nine-node tetrahedron (EUST9) shown in Figure 3a. The element consists of vertex nodes $1, \ldots, 4$, mid-face nodes $5, \ldots, 8$ and center node 9. The element geometry is identical to that of the eight-node tetrahedron in Ref. 1 with the addition of the center node. The coordinates of virtual mid-edge nodes $\hat{5}, \ldots, \hat{10}$ are constrained to those of the vertex nodes by the equations

$$
\begin{aligned}
& x_{i \hat{5}}=\left(x_{i 1}+x_{i 2}\right) / 2 \quad x_{i \hat{6}}=\left(x_{i 2}+x_{i 3}\right) / 2 \quad x_{i \hat{\imath}}=\left(x_{i 3}+x_{i 1}\right) / 2 \\
& x_{i \hat{\AA}}=\left(x_{i 1}+x_{i 4}\right) / 2 \quad x_{i \hat{9}}=\left(x_{i 2}+x_{i 4}\right) / 2 \quad x_{i \hat{1} 0}=\left(x_{i 3}+x_{i 4}\right) / 2
\end{aligned}
$$

The six virtual nodes are included simply to permit the decomposition of the tetrahedron into four hexahedra. Constraints in the same form as Eqs. (8-9) hold for the displacements of nodes $\hat{5}, \ldots, \hat{10}$.

The tetrahedron is geometrically decomposed into four hexahedra specified by the nodal

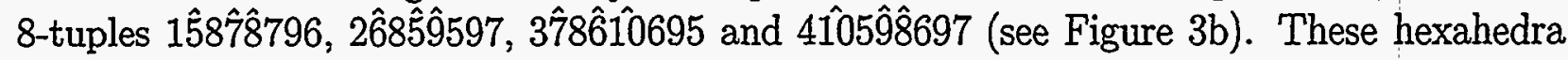
are designated by $1,2,3$ and 4 , respectively. Using the chain rule for differentiation along with Eqs. (3), (8) and (9), the $B$ matrices of the four hexahedra are given by

$$
B_{1}=\hat{B}_{1} I_{1} \quad B_{2}=\hat{B}_{2} I_{2} \quad B_{3}=\hat{B}_{3} I_{3} \quad B_{4}=\hat{B}_{4} I_{4}
$$

where $\hat{B}_{1}, \ldots, \hat{B}_{4}$ are the $B$ matrices of the four hexahedra calculated using Eq. (3). The matrices $I_{1}, \ldots, I_{4}$ take into account the constraints on nodes $\hat{5}, \ldots, \hat{10}$ (see Appendix).

Consider a distributed load on face 124 that varies linearly between nodes 1,2 and 4 . Assuming node 7 is centered between nodes 1,2 and 4, the equivalent nodal forces are given 
by

$$
\begin{aligned}
& f_{1}=\left[31 p_{1}+13\left(p_{2}+p_{4}\right)\right] A / 216 \\
& f_{2}=\left[31 p_{2}+13\left(p_{4}+p_{1}\right)\right] A / 216 \\
& f_{4}=\left[31 p_{4}+13\left(p_{1}+p_{2}\right)\right] A / 216 \\
& f_{7}=5\left(p_{1}+p_{2}+p_{4}\right) A / 72
\end{aligned}
$$

where $p_{1}, p_{2}$ and $p_{4}$ are values of the distributed load at nodes 1,2 and 4 and $A$ is the area of face 124. Similar expressions hold for the other three faces of the element. For $p_{1}=p_{2}=p_{4}=p$, Eqs. (11-14) simplify to

$$
f_{1}=19 p A / 72 \quad f_{2}=19 p A / 72 \quad f_{4}=19 p A / 72 \quad f_{7}=5 p A / 24
$$

\subsection{Eleven-Node Tetrahedron}

Consider the enhanced uniform strain eleven-node tetrahedron (EUST11) shown in Figure 4a. The element consists of vertex nodes $1, \ldots, 4$, mid-edge nodes $5, \ldots, 10$ and center node 11. The element geometry is identical to that of a standard ten-node tetrahedron with the addition of the center node. The coordinates of virtual mid-face nodes $\hat{5}, \ldots, \hat{8}$ are constrained to those of the vertex and mid-edge nodes by the equations

$$
\begin{aligned}
& x_{i \hat{\overline{5}}}=(1-\alpha)\left(x_{i 2}+x_{i 3}+x_{i 4}\right) / 3+\alpha\left(x_{i 6}+x_{i 10}+x_{i 9}\right) / 3 \\
& x_{i \hat{\hat{\sigma}}}=(1-\alpha)\left(x_{i 3}+x_{i 1}+x_{i 4}\right) / 3+\alpha\left(x_{i 7}+x_{i 8}+x_{i 10}\right) / 3 \\
& x_{i \hat{7}}=(1-\alpha)\left(x_{i 1}+x_{i 2}+x_{i 4}\right) / 3+\alpha\left(x_{i 5}+x_{i 9}+x_{i 8}\right) / 3 \\
& x_{i \hat{\mathrm{B}}}=(1-\alpha)\left(x_{i 2}+x_{i 1}+x_{i 3}\right) / 3+\alpha\left(x_{i 5}+x_{i 7}+x_{i 6}\right) / 3
\end{aligned}
$$

where $\alpha$ is a scalar. Again, the four virtual nodes are included simply to permit the decomposition of the tetrahedron into four hexahedra. Constraints in the same form as Eqs. (16-19) hold for the displacements of nodes $\hat{5}, \ldots, \hat{8}$.

The tetrahedron is geometrically decomposed into four hexahedra specified by the nodal 8-tuples $15 \hat{8} 78 \hat{7} 11 \hat{\hat{b}}, 26 \hat{8} 59 \hat{5} 11 \hat{7}, 37 \hat{8} 610 \hat{6} 11 \hat{5}$ and $410 \hat{5} 98 \hat{6} 11 \hat{7}$ (see Figure 4b). These hexahedra are designated by $1,2,3$ and 4 , respectively. Elements of the $B$ matrices of the four hexahedra are given by Eqs. (10) with definitions of the matrices $I_{1}, \ldots, I_{4}$ provided in the Appendix.

Consider a distributed load on face 124 that varies linearly between nodes 1,2 and 4 . Assuming nodes 5,8 and 9 are centered between the vertex nodes, the equivalent nodal forces are given by

$$
\begin{aligned}
& f_{1}=\left[323 p_{1} / 3456+253\left(p_{2}+p_{4}\right) / 6912-5 \alpha\left(p_{1}+p_{2}+p_{4}\right) / 216\right] A \\
& f_{2}=\left[323 p_{2} / 3456+253\left(p_{4}+p_{1}\right) / 6912-5 \alpha\left(p_{1}+p_{2}+p_{4}\right) / 216\right] A \\
& f_{4}=\left[323 p_{4} / 3456+253\left(p_{1}+p_{2}\right) / 6912-5 \alpha\left(p_{1}+p_{2}+p_{4}\right) / 216\right] A \\
& f_{5}=\left[35 p_{4} / 1728+253\left(p_{1}+p_{2}\right) / 3456+5 \alpha\left(p_{1}+p_{2}+p_{4}\right) / 216\right] A \\
& f_{9}=\left[35 p_{1} / 1728+253\left(p_{2}+p_{4}\right) / 3456+5 \alpha\left(p_{1}+p_{2}+p_{4}\right) / 216\right] A \\
& f_{8}=\left[35 p_{2} / 1728+253\left(p_{4}+p_{1}\right) / 3456+5 \alpha\left(p_{1}+p_{2}+p_{4}\right) / 216\right] A
\end{aligned}
$$


where $p_{1}, p_{2}$ and $p_{4}$ are values of the distributed load at nodes 1,2 and 4 and $A$ is the area of face 124. Similar expressions hold for the other three faces of the element. For $p_{1}=p_{2}=p_{4}=p$, Eqs. $(20-25)$ simplify to

$$
\begin{array}{lll}
f_{1}=(1 / 6-5 \alpha / 72) p A & f_{2}=(1 / 6-5 \alpha / 72) p A & f_{4}=(1 / 6-5 \alpha / 72) p A \\
f_{5}=(1 / 6+5 \alpha / 72) p A & f_{9}=(1 / 6+5 \alpha / 72) p A & f_{8}=(1 / 6+5 \alpha / 72) p A
\end{array}
$$

In contrast, the equivalent nodal forces for a tetrahedron with quadratic interpolation are given by

$$
\begin{array}{ccc}
f_{1}=0 & f_{2}=0 & f_{4}=0 \\
f_{5}=p A / 3 & f_{9}=p A / 3 & f_{8}=p A / 3
\end{array}
$$

The present formulation permits a constant pressure to be distributed in a continuously varying manner between the vertex and mid-edge nodes. This is accomplished simply by varying the scalar $\alpha$.

\subsection{Hourglass Control}

A general method of hourglass control is presented here that is applicable to all the element types considered in the study. A similar method that is spatially isotropic is given in Ref. 1. The purpose of hourglass control is to remove spurious zero energy modes from an element. We presently only consider hourglass stiffness, but one could easily include hourglass damping for problems in dynamics.

Let $u_{i I}$ denote the nodal displacements of an element and define

$$
d_{i}=\left[\begin{array}{llll}
u_{i 1} & u_{i 2} & \ldots & u_{i N}
\end{array}\right]^{T}
$$

where $N$ is the number of nodes in the element. The vector $d_{i}$ can be expressed as

$$
d_{i}=\Phi q_{i}+\hat{\Phi} \hat{q}_{i}
$$

where

$$
\Phi=\left[\begin{array}{cccc}
1 & \bar{x}_{11} & \bar{x}_{21} & \bar{x}_{31} \\
1 & \bar{x}_{12} & \bar{x}_{22} & \bar{x}_{32} \\
\vdots & \vdots & \vdots & \vdots \\
1 & \bar{x}_{1 N} & \bar{x}_{2 N} & \bar{x}_{3 N}
\end{array}\right]
$$

and

$$
\bar{x}_{i I}=x_{i I}-\frac{1}{N} \sum_{J=1}^{N} x_{i J}
$$

Premultiplying Eq. (31) by $\Phi^{T}$ and solving for $q_{i}$ yields

$$
q_{i}=\left(\Phi^{T} \Phi\right)^{-1} \Phi^{T} d_{i}
$$


Substituting Eq. (35) into Eq. (31) leads to

$$
\hat{\Phi} \hat{q}_{i}=\left[I-\Phi\left(\Phi^{T} \Phi\right)^{-1} \Phi^{T}\right] d_{i}
$$

In order to obtain a method that includes the effects of spatial scaling, i.e. element aspect ratios, it is useful to consider the square matrix $S$ consisting of rows and columns two through four of $\Phi^{T} \Phi$. It follows from Eq. (32) that

$$
S=\left[\begin{array}{lll}
s_{11} & s_{12} & s_{31} \\
s_{12} & s_{22} & s_{23} \\
s_{31} & s_{23} & s_{33}
\end{array}\right]
$$

where

$$
\begin{array}{ll}
s_{11}=\sum_{I=1}^{N} \bar{x}_{1 I} \bar{x}_{1 I}, & s_{22}=\sum_{I=1}^{N} \bar{x}_{2 I} \bar{x}_{2 I}, \quad s_{33}=\sum_{I=1}^{N} \bar{x}_{3 I} \bar{x}_{3 I} \\
s_{12}=\sum_{I=1}^{N} \bar{x}_{1 I} \bar{x}_{2 I}, & s_{23}=\sum_{I=1}^{N} \bar{x}_{2 I} \bar{x}_{3 I}, \quad s_{31}=\sum_{I=1}^{N} \bar{x}_{3 I} \bar{x}_{1 I}
\end{array}
$$

The eigen-decomposition of $S$ can be expressed as

$$
S=\Psi \Theta \Psi^{T}
$$

where the columns of $\Psi$ are orthonormal and $\Theta$ is a diagonal matrix of positive eigenvalues. It is noted that $\Psi$ and $\Theta$ can be calculated efficiently in closed-form.

Characteristic lengths $l_{i}$ are defined in terms of the eigenvalues of $S$ as

$$
l_{i}=\sqrt{\theta_{i}}
$$

The strain energy associated with hourglass stiffness is formulated as

$$
U_{h}=\epsilon G_{h} \sum_{i=1}^{3} \kappa_{i} \tilde{d}_{i}^{T} \tilde{d}_{i} / 2
$$

where $\epsilon$ is a positive scalar, $G_{h}$ is a material modulus, and

$$
\tilde{d}_{i}=\psi_{1 i}\left(\hat{\Phi} \hat{q}_{1}\right)+\psi_{2 i}\left(\hat{\Phi} \hat{q}_{2}\right)+\psi_{3 i}\left(\hat{\Phi} \hat{q}_{3}\right)
$$

The selection of $\kappa_{i}=V^{1 / 3}$ in Ref. 1 results in a spatially isotropic form of hourglass control. In the present study, we choose $\kappa_{i}$ as

$$
\begin{aligned}
& \kappa_{1}=\frac{l_{2} l_{3}}{l_{1}} \max \left[\min \left(1,\left(l_{1} / l_{2}\right)^{4},\left(l_{1} / l_{3}\right)^{4}\right), 10^{-6}\right] \\
& \kappa_{2}=\frac{l_{3} l_{1}}{l_{2}} \max \left[\min \left(1,\left(l_{2} / l_{3}\right)^{4},\left(l_{2} / l_{1}\right)^{4}\right), 10^{-6}\right] \\
& \kappa_{3}=\frac{l_{1} l_{2}}{l_{3}} \max \left[\min \left(1,\left(l_{3} / l_{1}\right)^{4},\left(l_{3} / l_{2}\right)^{4}\right), 10^{-6}\right]
\end{aligned}
$$


Notice that the leading terms on the right hand sides of Eqs. (44-46) are proportional to the axial stiffnesses of a rectangular parallelepiped with edge lengths $l_{1}, l_{2}$ and $l_{3}$. The terms raised to the fourth power are proportional to the ratios of bending to axial stiffnesses. The $10^{-6}$ terms are included so that at least a small fraction of the axial stiffness is used for hourglass control.

The nodal forces $f_{i h I}$ associated with hourglass stiffness are obtained by differentiating $U_{h}$ with respect to $d_{i}$ :

$$
f_{i h I}=\frac{\partial U_{h}}{\partial u_{i I}}
$$

Substituting Eqs. (36) and (42-43) into Eq. (47) leads to

$$
\left[\begin{array}{l}
f_{1 h I} \\
f_{2 h I} \\
f_{3 h I}
\end{array}\right]=\epsilon G_{h} \sum_{J=1}^{N} a_{I J}\left[\begin{array}{lll}
p_{11} & p_{12} & p_{31} \\
p_{12} & p_{22} & p_{23} \\
p_{31} & p_{23} & p_{33}
\end{array}\right]\left[\begin{array}{l}
u_{1 J} \\
u_{2 J} \\
u_{3 J}
\end{array}\right]
$$

where

$$
\begin{aligned}
a_{I J} & =\delta_{I J}-\left(\frac{1}{N}+\frac{\tilde{x}_{1 I} \tilde{x}_{1 J}}{\theta_{1}}+\frac{\tilde{x}_{2 I} \tilde{x}_{2 J}}{\theta_{2}}+\frac{\tilde{x}_{3 I} \tilde{x}_{3 J}}{\theta_{3}}\right) \\
\tilde{x}_{i L} & =\psi_{1 i} \bar{x}_{1 L}+\psi_{2 i} \bar{x}_{2 L}+\psi_{3 i} \bar{x}_{3 L} \\
p_{i j} & =\kappa_{1} \psi_{i 1} \psi_{j 1}+\kappa_{2} \psi_{i 2} \psi_{j 2}+\kappa_{3} \psi_{i 3} \psi_{j 3}
\end{aligned}
$$

and $\delta_{I J}$ is the Kronecker delta.

It follows from Eqs. $(36,42-43,47)$ that the hourglass control forces are orthogonal to $\Phi q_{i}$. In other words, hourglass stiffness does not cause any restoring forces if the nodal displacements are consistent with a linear displacement field, the desired result. Meshes of elements presented in Sections 2.1-2.3 that use this method of hourglass control pass first-order patch tests exactly.

\section{Example Problems}

All the example problems in this section assume small and static deformations of a linear, elastic, isotropic material with Young's modulus $E$ and Poisson's ratio $\nu$. Equations for the stiffness matrices of the nine-node tetrahedron and the eleven-node tetrahedron are presented below. Recall that the seven-node triangle is simply the union of three quadrilaterals.

Let,

$$
d=\left[\begin{array}{llllllllll}
u_{11} & u_{21} & u_{31} & u_{12} & u_{22} & u_{32} & \ldots & u_{1 N} & u_{2 N} & u_{3 N}
\end{array}\right]^{T}
$$

Corresponding to $d$ are stiffness matrices associated with elastic strains and hourglass control. With reference to Eq. (4), the stiffness matrix for elastic strains is given by

$$
K^{e s}=\sum_{j=1}^{4} C_{j}^{T} H C_{j} / V_{j}
$$


where $V_{j}$ is the volume of hexahedron $j$ and

$$
\begin{gathered}
H=\left[\begin{array}{cccccc}
2 G+\lambda & \lambda & \lambda & 0 & 0 & 0 \\
\lambda & 2 G+\lambda & \lambda & 0 & 0 & 0 \\
\lambda & \lambda & 2 G+\lambda & 0 & 0 & 0 \\
0 & 0 & 0 & G & 0 & 0 \\
0 & 0 & 0 & 0 & G & 0 \\
0 & 0 & 0 & 0 & 0 & G
\end{array}\right] \\
G=\frac{E}{2(1+\nu)} \\
\lambda=\frac{E \nu}{(1+\nu)(1-2 \nu)}
\end{gathered}
$$

The nonzero elements of the matrices $C_{j}$ are given by

$$
\begin{array}{lll}
C_{j 1,3(I-1)+1}=B_{j 1 I} & C_{j 2,3(I-1)+2}=B_{j 2 I} & C_{j 3,3(I-1)+3}=B_{j 3 I} \\
C_{j 4,3(I-1)+1}=B_{j 2 I} & C_{j 5,3(I-1)+2}=B_{j 3 I} & C_{j 6,3(I-1)+3}=B_{j 1 I} \\
C_{j 4,3(I-1)+2}=B_{j 1 I} & C_{j 5,3(I-1)+3}=B_{j 2 I} & C_{j 6,3(I-1)+1}=B_{j 3 I}
\end{array}
$$

With reference to Eq. (48), we see that the elements of the hourglass stiffness matrix $K^{\text {hg }}$ in rows $3(I-1)+1$ to $3 I$ and columns $3(J-1)+1$ to $3 J$ are given by $K_{I J}^{h g}$ where

$$
K_{I J}^{h g}=\epsilon G_{h} a_{I J}\left[\begin{array}{lll}
p_{11} & p_{12} & p_{31} \\
p_{12} & p_{22} & p_{23} \\
p_{31} & p_{23} & p_{33}
\end{array}\right]
$$

The stiffness matrix of the element is the sum of $K^{e s}$ and $K^{\text {hg }}$. That is,

$$
K=K^{e s}+K^{h g}
$$

Element stiffness matrices are assembled as is done conventionally to form the stiffness matrix of the entire model.

All the example problems are for a material with $E=10^{7}$ using hourglass control specified by $\epsilon=0.05$ and $G_{h}=G$. A value of $\alpha=6 / 5$ is used for the eleven-node tetrahedron. This value of $\alpha$ causes a constant pressure on an element face to be distributed 1/12 at each of the vertex nodes and 1/4 at each of the mid-edge nodes (see Eqs. 26-27). This value of $\alpha$ is also suited for purposes of comparison with an element from a commercial finite element code [4]. The values of $\alpha$ reported subsequently for the elements of Ref. 1 cause mid-edge and mid-face nodes to lie on the element boundaries.

Comparisons are made in the example problems between triangular, quadrilateral, tetrahedral and hexahedral elements. For the 2D problems, a rectangular structure of unit thickness bounded by $x_{i}=0$ and $x_{i}=h_{i}$ for $i=1,2$ is used. A cubic structure bounded by $x_{i}=0$ 
and $x_{i}=h_{i}$ for $i=1,2,3$ is used for the $3 \mathrm{D}$ problems. The meshes used in the problems can be characterized by the number of elements $n$ per edge of the boundary. Meshes with $n=4$ are shown in Figure 5. Each of the $n^{3}$ cubic blocks in the tetrahedral meshes is composed of five tetrahedra. Thus, the mesh shown in Figure $5 \mathrm{c}$ has 320 elements. The mid-edge and mid-face nodes of the triangular and tetrahedral elements are not shown in Figure 5.

Although the number of nodes per element is greater for the eleven-node tetrahedron than the nine-node tetrahedron, there are generally fewer numbers of nodes for meshes of eleven-node tetrahedra. For example, the meshes used for the 3D problems have a total of $12 n^{3}+12 n^{2}+6 n+1$ nodes for the eleven-node tetrahedron compared with $16 n^{3}+9 n^{2}+3 n+1$ nodes for the nine-node tetrahedron. The reason for the difference is that a greater number of elements can share mid-edge nodes than mid-face nodes. At most only two elements can have the same mid-face node for meshes of nine-node tetrahedra.

\subsection{Example 1}

The first example deals with the classic problem of pure bending. The applied tractions on the face defined by $x_{1}=h_{1}$ are specified as

$$
\sigma_{11}\left(x_{2}, x_{3}\right)=E\left(h_{2} / 2-x_{2}\right) / R
$$

The displacement boundary conditions for the $2 \mathrm{D}$ problem are given by

$$
\begin{array}{r}
u_{1}\left(0, x_{2}\right)=0 \\
u_{2}(0,0)=0
\end{array}
$$

and for the 3D problem

$$
\begin{array}{r}
u_{1}\left(0, x_{2}, x_{3}\right)=0 \\
u_{2}(0,0,0)=0 \\
u_{3}(0,0,0)=0 \\
u_{2}\left(0,0, h_{3}\right)=0
\end{array}
$$

The solution for $2 \mathrm{D}$ plane stress has

$$
\begin{aligned}
& u_{1}\left(x_{1}, x_{2}\right)=x_{1}\left(h_{2} / 2-x_{2}\right) / R \\
& u_{2}\left(x_{1}, x_{2}\right)=\frac{1}{2 R}\left[x_{1}^{2}+\nu\left[\left(h_{2} / 2-x_{2}\right)^{2}-h_{2}^{2} / 4\right]\right]
\end{aligned}
$$

while the $3 \mathrm{D}$ elasticity solution is given by

$$
\begin{aligned}
& u_{1}\left(x_{1}, x_{2}, x_{3}\right)=x_{1}\left(h_{2} / 2-x_{2}\right) / R \\
& u_{2}\left(x_{1}, x_{2}, x_{3}\right)=\frac{1}{2 R}\left[x_{1}^{2}+\nu\left[\left(h_{2} / 2-x_{2}\right)^{2}-\left(x_{3}^{2}-h_{3} x_{3}+h_{2}^{2} / 4\right)\right]\right] \\
& u_{3}\left(x_{1}, x_{2}, x_{3}\right)=\nu\left(x_{2} x_{3}-h_{3} x_{2} / 2-h_{2} x_{3} / 2\right) / R
\end{aligned}
$$


For purposes of comparison with the exact solutions, tip displacement ratios for the $2 \mathrm{D}$ and $3 \mathrm{D}$. problems are defined as

$$
\bar{u}=u_{2}^{F E}\left(h_{1}, h_{2} / 2\right) / u_{2}\left(h_{1}, h_{2} / 2\right) .
$$

and

$$
\bar{u}=u_{2}^{F E}\left(h_{1}, h_{2} / 2, h_{3} / 2\right) / u_{2}\left(h_{1}, h_{2} / 2, h_{3} / 2\right)
$$

where $u^{F E}$ denotes the finite element solution.

Calculated values of $\bar{u}$ for 2D plane stress are shown in Table 1 for meshes with 4 elements per edge $(n=4)$. Results are shown for the three-node constant strain triangle (CST3), the four-node uniform strain quadrilateral (USQ4), the six-node uniform strain triangle of Ref. 1 with $\alpha=1 / 2$ (UST6), and the seven-node enhanced uniform strain triangle of Section 2.1 (EUST7). It is clear that none of the uniform strain elements suffer from volumetric locking or parasitic shear for the applied boundary conditions. In contrast, the constant strain triangle is much too stiff for all values of $\nu$.

Plots of the energy norm (see Ref. 3 ) for the different element types are shown in Figure 6 for $\nu=0.3$. The results for the enhanced uniform strain triangle are clearly more accurate than the others. Slopes near unity of the lines in the figure are consistent with the convergence rate of linear displacement/constant stress elements. The slope less than unity for CST4 indicates that the meshes used are too coarse for asymptotic convergence to occur. As the mesh is refined further, the slope of the energy norm for CST4 approaches unity.

Calculated values of $\bar{u}$ for the 3D problem are shown in Table 2 for meshes with $n=4$. Results are shown for the four-node constant strain tetrahedron (CST4), the eight-node uniform strain hexahedron (USH8), the eight-node uniform strain tetrahedron of Ref. 1 with $\alpha=1 / 3$ (UST8), the ten-node uniform strain hexahedron of Ref. 1 with $\alpha=3 / 4$ (UST10), the nine-node enhanced uniform strain tetrahedron of Section 2.2 (EUST9), and the elevennode enhanced uniform strain tetrahedron of Section 2.3 (EUST11).

It is clear from Table 2 that eleven-node enhanced uniform strain tetrahedron EUST11 accurately predicts the tip displacement. In contrast, the enhanced uniform strain nine-node tetrahedron EUST9 performs much more poorly than its uniform strain counterpart UST8. Values of the tip displacement ratio $\bar{u}$ less much than unity for CST4 and EUST9 indicate that the elements are too stiff.

Table 3 reports values of the total strain energy $U_{\text {tot }}$ for the different 3D element types. For purposes of comparison, the exact value of $U_{\text {tot }}$ from the elasticity solution

$$
U_{\text {tot }}=h_{1} h_{2}^{3} h_{3} E /\left(24 R^{2}\right)
$$

is also reported. Notice that the values of $U_{\text {tot }}$ for CST4 and EUST9 are consistently lower than the exact value. This trend is in agreement with the observation these elements are too stiff.

The stiff behavior of EUST9 can be explained by noting that six constraints restrict the motion of virtual mid-edge nodes $\hat{5}, \ldots, \hat{10}$ (see Eqs. 8-9). Along any edge, element deformations are constrained to vary linearly between the two vertex nodes. It appears from 
this example that these constraints cause the element to be too stiff. It was found that a reformulated EUST9 element which uses single point integration "mean quadrature" for the deviatoric strain energy improves the element performance, but offers no clear advantage over UST8. The enhanced uniform strain element EUST11 only has four constraints that restrict the motion of virtual mid-face nodes $\hat{5}, \ldots, \hat{8}$ (see Eqs. 16-19). In addition, deformations may vary bilinearly along the element edges.

Plots of the energy norm for the different element types are shown in Figure 7 for $\nu=0.3$. The results for the eleven-node enhanced uniform strain element EUST11 are clearly more accurate than the others. The slope much less than unity for CST4 indicates that the meshes are not of sufficient refinement for asymptotic convergence to occur.

\subsection{Example 2}

The second example considers a problem in which the displacements of all nodes on the boundary are specified. For the 2D problem, nodes on the boundaries $x_{i}=0$ and $x_{i}=h_{i}$ $(i=1,2)$ are subjected to the enforced displacements

$$
\begin{aligned}
& u_{1}\left(x_{1}, x_{2}, x_{3}\right)=a\left(x_{2}^{2}-x_{1}^{2}+2 x_{1} x_{2}\right) \\
& u_{2}\left(x_{1}, x_{2}, x_{3}\right)=a\left(x_{1}^{2}-x_{2}^{2}+2 x_{2} x_{1}\right)
\end{aligned}
$$

where $a$ is a constant and the plane strain assumption is applied. The nodal displacements on the boundaries $x_{1}=0$ and $x_{i}=h_{i}(i=1,2,3)$ for the 3D problem are specified as

$$
\begin{aligned}
& u_{1}\left(x_{1}, x_{2}, x_{3}\right)=a\left(x_{2}^{2}+x_{3}^{2}-2 x_{1}^{2}+2 x_{1} x_{2}+2 x_{1} x_{3}+5 x_{2} x_{3}\right) \\
& u_{2}\left(x_{1}, x_{2}, x_{3}\right)=a\left(x_{3}^{2}+x_{1}^{2}-2 x_{2}^{2}+2 x_{2} x_{3}+2 x_{2} x_{1}+5 x_{3} x_{1}\right) \\
& u_{3}\left(x_{1}, x_{2}, x_{3}\right)=a\left(x_{1}^{2}+x_{2}^{2}-2 x_{3}^{2}+2 x_{3} x_{1}+2 x_{3} x_{2}+5 x_{1} x_{2}\right)
\end{aligned}
$$

The elasticity solutions to the 2D and 3D boundary value problems are given by Eqs. (77-81) as well. The total strain energies for the $2 \mathrm{D}$ and $3 \mathrm{D}$ problems are given, respectively, by

$$
U_{\text {tot }}=16 h_{1} h_{2}\left(h_{1}^{2}+h_{2}^{2}\right) G a^{2} / 3
$$

and

$$
U_{\text {tot }}=6 h_{1} h_{2} h_{3}\left[5\left(h_{1}^{2}+h_{2}^{2}+h_{3}^{2}\right)+3\left(h_{1} h_{2}+h_{2} h_{3}+h_{3} h_{1}\right)\right] G a^{2}
$$

One can confirm that the elasticity solutions have no volumetric strain. That is,

$$
\frac{\partial u_{1}}{\partial x_{1}}+\frac{\partial u_{2}}{\partial x_{2}}+\frac{\partial u_{3}}{\partial x_{3}}=0
$$

Consequently, the exact value of the volumetric strain energy $U_{\text {vol }}$ is zero.

Calculated values of $U_{\text {tot }}$ for the $2 \mathrm{D}$ plane strain problem are shown in Table 4 . In addition to the 2D elements mentioned previously, Table 4 includes results for two triangular elements from a commercial finite element code [4]. Element type CPE6 is a six-node plane strain triangular element with quadratic interpolation. Element type CPE6M is described as a modified six-node plane strain triangular element. 
The only element type that appears to suffer from volumetric locking is the constant strain triangle. Results for element type CPE6 are in perfect agreement with the exact solution because the quadratic elements can approximate the elasticity solution exactly. Notice that the results presented for element types EUST7 and CPE6M are identical. Plots of the energy norm for several 2D element types are shown in Figure 8 for $\nu=0.499$. Again, the plots show that EUST7 is significantly more accurate than the other element types.

Calculated values of $U_{\text {tot }}$ and $U_{v o l}$ for the 3D problem are shown in Tables 5 and 6 , respectively. In addition to the $3 \mathrm{D}$ elements mentioned previously, Table 5 includes results for two different tetrahedral elements described in Ref. 4. Element type C3D10 is a ten-node tetrahedron with quadratic interpolation. Element type C3D10M is described as a modified ten-node tetrahedron.

It is evident from Table 6 that the constant strain tetrahedron suffers from volumetric locking for the applied boundary conditions. Element types C3D10M, UST10 and EUST11 also display locking behavior, but to a much lesser extent. The results for element type C3D10 are in perfect agreement with the exact solution because the quadratic elements can approximate the elasticity solution exactly. Notice that the results presented for element types EUST11 and C3D10M are identical.

The locking of element types CST4, UST10 and EUST11 is caused by overly stringent displacement boundary conditions. For example, all the nodal displacements of UST10 elements at the corners of the cube are specified. As a consequence, volume changes in the corner elements are unavoidable. For $\nu=0.49999$, over 99.9999 percent of the volumetric strain energy for the UST10 and EUST11 elements is contained in elements that have a face on the boundary. Of this percentage, approximately one half is contained in the eight corner nodes. Element types UST8 and EUST9 do not lock because there are unconstrained midface nodes to accommodate zero volume change. For the all-hexahedral mesh, the volumetric strain energy is identically zero for all values of $\nu$. This remarkable result only holds if none of the hexahedral elements are skewed.

\subsection{Example 3}

Rather than prescribing the displacement of all nodes on the boundary, alternative boundary conditions are considered in which surface tractions corresponding to the $3 \mathrm{D}$ elasticity solution (see Eqs. 79-81) are applied to the six sides of the cube. Rigid body motion is restricted by the displacement boundary conditions

$$
\begin{array}{ccc}
u_{1}(0,0,0)=0 & u_{2}(0,0,0)=0 & u_{3}(0,0,0)=0 \\
u_{1}(0,10,0)=100 a & u_{2}(0,0,10)=100 a & u_{3}(10,0,0)=100 a
\end{array}
$$

Calculated values of $U_{\text {tot }}$ and $U_{v o l}$ are shown in Tables 7 and 8. Notice from the results that none of the elements suffer from volumetric locking.

Plots of the energy norm for the different element types are shown in Figure 9 for $\nu=$ 0.499 . Again, the eleven-node enhanced uniform strain element is significantly more accurate than the other element types. The slope much less than unity of the energy norm for CST4 indicates that the meshes used are too coarse for asymptotic convergence. 


\section{Conclusions}

A family of enhanced uniform strain triangular and tetrahedral finite elements is presented. Element types considered in the study include a seven-node triangle, nine-node tetrahedron, and eleven-node tetrahedron. By allowing more than a single state of uniform strain within each element, significant improvements in accuracy are obtained for the sevennode triangle and the eleven-node tetrahedron over their uniform strain counterparts. In addition, the number of hourglass modes for the enhanced elements is reduced significantly.

The formulation for the eleven-node tetrahedron allows a uniform pressure to be distributed in a continuously varying manner between vertex and mid-edge nodes. In contrast, the standard quadratic tetrahedron distributes a uniform pressure entirely at the vertex nodes. This flexibility in the element formulation may prove useful for applications involving contact where a uniform normal stiffness is desirable.

The performance of the nine-node tetrahedron is much worse than its uniform strain counterpart, the eight-node tetrahedron. Improvements in the element performance can be obtained by using only single point integration "mean quadrature" for the deviatoric portion of the strain energy, but the reformulated element has no clear advantage over the eight-node tetrahedron.

The disappointing performance of the nine-node tetrahedron is caused by the presence of six constraints that restrict the motion of the virtual mid-edge nodes of the element. Element deformations are constrained to vary linearly between the two vertex nodes defining an edge. These constraints result in an element that is too stiff when greater than single point integration is used for the shear energy. In contrast, there are no constraints on the mid-edge nodes of the seven-node triangle and the eleven-node tetrahedron.

Results presented for the seven-node triangle of Section 2.1 are identical to those of a modified six-node triangle available in a commercial finite element code. Identical results are also presented for a special case $(\alpha=6 / 5)$ of the eleven-node tetrahedron of Section 2.3 and the modified ten-node tetrahedron of Ref. 4.

\section{Appendix}

Based on Eqs. (8-9), the constraint matrices for the nine-node tetrahedron are given by

$$
I_{1}=\left[\begin{array}{ccccccccc}
1 & 0 & 0 & 0 & 0 & 0 & 0 & 0 & 0 \\
1 / 2 & 1 / 2 & 0 & 0 & 0 & 0 & 0 & 0 & 0 \\
0 & 0 & 0 & 0 & 0 & 0 & 0 & 1 & 0 \\
1 / 2 & 0 & 1 / 2 & 0 & 0 & 0 & 0 & 0 & 0 \\
1 / 2 & 0 & 0 & 1 / 2 & 0 & 0 & 0 & 0 & 0 \\
0 & 0 & 0 & 0 & 0 & 0 & 1 & 0 & 0 \\
0 & 0 & 0 & 0 & 0 & 0 & 0 & 0 & 1 \\
0 & 0 & 0 & 0 & 0 & 1 & 0 & 0 & 0
\end{array}\right]
$$




$$
\begin{aligned}
I_{2} & =\left[\begin{array}{ccccccccc}
0 & 1 & 0 & 0 & 0 & 0 & 0 & 0 & 0 \\
0 & 1 / 2 & 1 / 2 & 0 & 0 & 0 & 0 & 0 & 0 \\
0 & 0 & 0 & 0 & 0 & 0 & 0 & 1 & 0 \\
1 / 2 & 1 / 2 & 0 & 0 & 0 & 0 & 0 & 0 & 0 \\
0 & 1 / 2 & 0 & 1 / 2 & 0 & 0 & 0 & 0 & 0 \\
0 & 0 & 0 & 0 & 1 & 0 & 0 & 0 & 0 \\
0 & 0 & 0 & 0 & 0 & 0 & 0 & 0 & 1 \\
0 & 0 & 0 & 0 & 0 & 0 & 1 & 0 & 0
\end{array}\right] \\
I_{3} & =\left[\begin{array}{ccccccccc}
0 & 0 & 1 & 0 & 0 & 0 & 0 & 0 & 0 \\
1 / 2 & 0 & 1 / 2 & 0 & 0 & 0 & 0 & 0 & 0 \\
0 & 0 & 0 & 0 & 0 & 0 & 0 & 1 & 0 \\
0 & 1 / 2 & 1 / 2 & 0 & 0 & 0 & 0 & 0 & 0 \\
0 & 0 & 1 / 2 & 1 / 2 & 0 & 0 & 0 & 0 & 0 \\
0 & 0 & 0 & 0 & 0 & 1 & 0 & 0 & 0 \\
0 & 0 & 0 & 0 & 0 & 0 & 0 & 0 & 1 \\
0 & 0 & 0 & 0 & 1 & 0 & 0 & 0 & 0
\end{array}\right] \\
I_{4} & =\left[\begin{array}{ccccccccc}
0 & 0 & 0 & 1 & 0 & 0 & 0 & 0 & 0 \\
0 & 0 & 1 / 2 & 1 / 2 & 0 & 0 & 0 & 0 & 0 \\
0 & 0 & 0 & 0 & 1 & 0 & 0 & 0 & 0 \\
0 & 1 / 2 & 0 & 1 / 2 & 0 & 0 & 0 & 0 & 0 \\
1 / 2 & 0 & 0 & 1 / 2 & 0 & 0 & 0 & 0 & 0 \\
0 & 0 & 0 & 0 & 0 & 1 & 0 & 0 & 0 \\
0 & 0 & 0 & 0 & 0 & 0 & 0 & 0 & 1 \\
0 & 0 & 0 & 0 & 0 & 0 & 1 & 0 & 0
\end{array}\right]
\end{aligned}
$$
by

Based on Eqs. (16-19), the constraint matrices for the eleven-node tetrahedron are given

$$
I_{1}=\left[\begin{array}{ccccccccccc}
1 & 0 & 0 & 0 & 0 & 0 & 0 & 0 & 0 & 0 & 0 \\
0 & 0 & 0 & 0 & 1 & 0 & 0 & 0 & 0 & 0 & 0 \\
w_{v} & w_{v} & w_{v} & 0 & w_{m} & w_{m} & w_{m} & 0 & 0 & 0 & 0 \\
0 & 0 & 0 & 0 & 0 & 0 & 1 & 0 & 0 & 0 & 0 \\
0 & 0 & 0 & 0 & 0 & 0 & 0 & 1 & 0 & 0 & 0 \\
w_{v} & w_{v} & 0 & w_{v} & w_{m} & 0 & 0 & w_{m} & w_{m} & 0 & 0 \\
0 & 0 & 0 & 0 & 0 & 0 & 0 & 0 & 0 & 0 & 1 \\
w_{v} & 0 & w_{v} & w_{v} & 0 & 0 & w_{m} & w_{m} & 0 & w_{m} & 0
\end{array}\right]
$$




$$
\begin{aligned}
I_{2} & =\left[\begin{array}{ccccccccccc}
0 & 1 & 0 & 0 & 0 & 0 & 0 & 0 & 0 & 0 & 0 \\
0 & 0 & 0 & 0 & 0 & 1 & 0 & 0 & 0 & 0 & 0 \\
w_{v} & w_{v} & w_{v} & 0 & w_{m} & w_{m} & w_{m} & 0 & 0 & 0 & 0 \\
0 & 0 & 0 & 0 & 1 & 0 & 0 & 0 & 0 & 0 & 0 \\
0 & 0 & 0 & 0 & 0 & 0 & 0 & 0 & 1 & 0 & 0 \\
0 & w_{v} & w_{v} & w_{v} & 0 & w_{m} & 0 & 0 & w_{m} & w_{m} & 0 \\
0 & 0 & 0 & 0 & 0 & 0 & 0 & 0 & 0 & 0 & 1 \\
w_{v} & w_{v} & 0 & w_{v} & w_{m} & 0 & 0 & w_{m} & w_{m} & 0 & 0
\end{array}\right] \\
I_{3} & =\left[\begin{array}{ccccccccccc}
0 & 0 & 1 & 0 & 0 & 0 & 0 & 0 & 0 & 0 & 0 \\
0 & 0 & 0 & 0 & 0 & 0 & 1 & 0 & 0 & 0 & 0 \\
w_{v} & w_{v} & w_{v} & 0 & w_{m} & w_{m} & w_{m} & 0 & 0 & 0 & 0 \\
0 & 0 & 0 & 0 & 0 & 1 & 0 & 0 & 0 & 0 & 0 \\
0 & 0 & 0 & 0 & 0 & 0 & 0 & 0 & 0 & 1 & 0 \\
w_{v} & 0 & w_{v} & w_{v} & 0 & 0 & w_{m} & w_{m} & 0 & w_{m} & 0 \\
0 & 0 & 0 & 0 & 0 & 0 & 0 & 0 & 0 & 0 & 1 \\
0 & w_{v} & w_{v} & w_{v} & 0 & w_{m} & 0 & 0 & w_{m} & w_{m} & 0
\end{array}\right] \\
I_{4} & =\left[\begin{array}{cccccccccccc}
0 & 0 & 0 & 1 & 0 & 0 & 0 & 0 & 0 & 0 & 0 \\
0 & 0 & 0 & 0 & 0 & 0 & 0 & 0 & 0 & 1 & 0 \\
0 & w_{v} & w_{v} & w_{v} & 0 & w_{m} & 0 & 0 & w_{m} & w_{m} & 0 \\
0 & 0 & 0 & 0 & 0 & 0 & 0 & 0 & 1 & 0 & 0 \\
0 & 0 & 0 & 0 & 0 & 0 & 0 & 1 & 0 & 0 & 0 \\
w_{v} & 0 & w_{v} & w_{v} & 0 & 0 & w_{m} & w_{m} & 0 & w_{m} & 0 \\
0 & 0 & 0 & 0 & 0 & 0 & 0 & 0 & 0 & 0 & 1 \\
w_{v} & w_{v} & 0 & w_{v} & w_{m} & 0 & 0 & w_{m} & w_{m} & 0 & 0
\end{array}\right]
\end{aligned}
$$

where $w_{v}=(1-\alpha) / 3$ and $w_{m}=\alpha / 3$. 


\section{References}

1. C. R. Dohrmann, S. W. Key, M. W. Heinstein and J. Jung, 'A Least Squares Approach for Uniform Strain Triangular and Tetrahedral Finite Elements', International Journal for Numerical Methods in Engineering, 42, 1181-1197 (1998).

2. D. P. Flanagan and T. Belytschko, 'A Uniform Strain Hexahedron and Quadrilateral with Orthogonal Hourglass Control', International Journal for Numerical Methods in Engineering, 17, 679-706 (1981).

3. O. C. Zienkiewicz and R. L. Taylor, The Finite Element Method, Vol. 1, 4th Ed., McGraw-Hill, New York, New York, 1989.

4. ABAQUS/Standard User's Manual (Version 5.7), Vol. 2, Hibbitt, Karlsson and Sorensen, Inc., (1997). 
Table 1: Tip displacement ratios for Example 3.1 (2D plane stress analysis, $h_{1}=10$, $\left.h_{2}=1, R=1 \times 10^{4}\right)$.

\begin{tabular}{|c|c|c|c|c|}
\hline$\nu$ & CST3 & USQ4 & UST6 & EUST7 \\
\hline 0.0 & 0.096 & 1.058 & 1.035 & 0.998 \\
0.1 & 0.104 & 1.059 & 1.033 & 0.998 \\
0.2 & 0.113 & 1.059 & 1.032 & 0.998 \\
0.3 & 0.121 & 1.059 & 1.030 & 0.998 \\
0.4 & 0.129 & 1.060 & 1.029 & 0.998 \\
0.499 & 0.137 & 1.060 & 1.027 & 0.998 \\
\hline
\end{tabular}

Table 2: Tip displacement ratios for Example 3.1 (3D analysis, $h_{1}=10, h_{2}=1, h_{3}=0.1$, $\left.R=1 \times 10^{4}\right)$.

\begin{tabular}{|c|c|c|c|c|c|c|}
\hline$\nu$ & CST4 & USH8 & UST8 & EUST9 & UST10 & EUST11 \\
\hline 0.0 & 0.0031 & 1.050 & 1.020 & 0.190 & 1.021 & 0.992 \\
0.1 & 0.0034 & 1.051 & 1.020 & 0.205 & 1.021 & 0.993 \\
0.2 & 0.0038 & 1.052 & 1.020 & 0.219 & 1.022 & 0.994 \\
0.3 & 0.0041 & 1.053 & 1.021 & 0.232 & 1.023 & 0.994 \\
0.4 & 0.0044 & 1.054 & 1.022 & 0.242 & 1.024 & 0.995 \\
0.499 & 0.0047 & 1.054 & 1.022 & 0.158 & 1.024 & 0.995 \\
\hline
\end{tabular}

Table 3: Total strain energy $U_{\text {tot }} \times 10^{3}$ for Example 3.1 (3D analysis, $h_{1}=10, h_{2}=1$, $h_{3}=0.1, R=1 \times 10^{4}$ ).

\begin{tabular}{|c|c|c|c|c|c|c|c|}
\hline$\nu$ & CST4 & USH8 & UST8 & EUST9 & UST10 & EUST11 & exact \\
\hline 0.0 & 0.01 & 4.32 & 4.21 & 0.86 & 4.21 & 4.09 & 4.17 \\
0.1 & 0.02 & 4.33 & 4.22 & 0.92 & 4.22 & 4.10 & 4.17 \\
0.2 & 0.02 & 4.34 & 4.23 & 0.98 & 4.23 & 4.10 & 4.17 \\
0.3 & 0.02 & 4.35 & 4.23 & 1.03 & 4.23 & 4.11 & 4.17 \\
0.4 & 0.02 & 4.36 & 4.24 & 1.06 & 4.24 & 4.11 & 4.17 \\
0.499 & 0.02 & 4.36 & 4.24 & 0.70 & 4.24 & 4.12 & 4.17 \\
\hline
\end{tabular}


Table 4: Total strain energy $U_{\text {tot }}$ for Example $3.2\left(2 \mathrm{D}\right.$ plane strain analysis, $h_{1}=10$,

$$
h_{2}=10, a=4 \times 10^{-6} \text { ). }
$$

\begin{tabular}{|c|c|c|c|c|c|c|c|}
\hline$\nu$ & CST3 & USQ4 & UST6 & EUST7 & CPE6 & CPE6M & exact \\
\hline 0.0 & 8.54 & 8.40 & 8.17 & 8.500 & 8.533 & 8.500 & 8.533 \\
0.1 & 7.77 & 7.64 & 7.45 & 7.728 & 7.758 & 7.728 & 7.758 \\
0.2 & 7.13 & 7.00 & 6.86 & 7.084 & 7.111 & 7.084 & 7.111 \\
0.3 & 6.60 & 6.46 & 6.35 & 6.539 & 6.564 & 6.539 & 6.564 \\
0.4 & 6.16 & 6.00 & 5.92 & 6.072 & 6.095 & 6.072 & 6.095 \\
0.499 & 9.91 & 5.60 & 5.55 & 5.671 & 5.693 & 5.671 & 5.693 \\
0.4999 & 47.4 & 5.60 & 5.55 & 5.667 & 5.689 & 5.667 & 5.689 \\
0.49999 & 422 & 5.60 & 5.55 & 5.667 & 5.689 & 5.667 & 5.689 \\
\hline
\end{tabular}

Table 5: Total strain energy $U_{\text {tot }}$ for Example 3.2 (3D analysis, $h_{1}=10, h_{2}=10, h_{3}=10$,

$$
\left.a=4 \times 10^{-6}\right) \text {. }
$$

\begin{tabular}{|c|c|c|c|c|c|c|c|c|c|}
\hline$\nu$ & CST4 & USH8 & UST8 & EUST9 & UST10 & EUST11 & C3D10 & C3D10M & exact \\
\hline 0.0 & 1160 & 1141 & 1139 & 1150 & 1141 & 1150 & 1152 & 1150 & 1152 \\
0.1 & 1056 & 1037 & 1036 & 1046 & 1038 & 1046 & 1047 & 1046 & 1047 \\
0.2 & 970 & 951 & 950 & 959 & 952 & 958.5 & 960.0 & 958.5 & 960.0 \\
0.3 & 899 & 878 & 878 & 885 & 879 & 884.8 & 886.2 & 884.8 & 886.2 \\
0.4 & 846 & 815 & 816 & 823 & 817 & 821.6 & 822.9 & 821.6 & 822.9 \\
0.499 & 2676 & 761 & 762 & 770 & 766 & 770.0 & 768.5 & 770.0 & 768.5 \\
0.4999 & $2 \mathrm{e} 4$ & 761 & 762 & 769 & 789 & 793.0 & 768.1 & 793.0 & 768.1 \\
0.49999 & $2 \mathrm{e} 5$ & 761 & 762 & 769 & 1023 & 1027 & 768.0 & 1027 & 768.0 \\
\hline
\end{tabular}

Table 6: Volumetric strain energy $U_{\text {vol }}$ for Example 3.2 (3D analysis, $h_{1}=10, h_{2}=10$, $h_{3}=10, a=4 \times 10^{-6}$ ).

\begin{tabular}{|c|c|c|c|c|c|c|c|}
\hline$\nu$ & CST4 & USH8 & UST8 & EUST9 & UST10 & EUST11 & exact \\
\hline 0.0 & 4.18 & 0 & 1.35 & 0.61 & 1.05 & 0.02 & 0 \\
0.1 & 5.17 & 0 & 1.19 & 0.68 & 0.95 & 0.02 & 0 \\
0.2 & 6.81 & 0 & 0.99 & 0.77 & 0.82 & 0.03 & 0 \\
0.3 & 10.0 & 0 & 0.75 & 0.87 & 0.64 & 0.04 & 0 \\
0.4 & 19.5 & 0 & 0.44 & 0.92 & 0.41 & 0.05 & 0 \\
0.499 & $2 \mathrm{e} 3$ & 0 & $6 \mathrm{e}-3$ & 0.04 & 2.61 & 2.61 & 0 \\
0.4999 & $2 \mathrm{e} 4$ & 0 & $6 \mathrm{e}-4$ & $4 \mathrm{e}-3$ & 26.0 & 26.0 & 0 \\
0.49999 & $2 \mathrm{e} 5$ & 0 & $6 \mathrm{e}-5$ & $4 \mathrm{e}-4$ & 260 & 260 & 0 \\
\hline
\end{tabular}


Table 7: Total strain energy $U_{\text {tot }}$ for Example 3.3 (3D analysis, $h_{1}=10, h_{2}=10, h_{3}=10$, $a=4 \times 10^{-6}$ ).

\begin{tabular}{|c|c|c|c|c|c|c|c|}
\hline$\nu$ & CST4 & USH8 & UST8 & EUST9 & UST10 & EUST11 & exact \\
\hline 0.0 & 1139 & 1156 & 1159 & 1149 & 1160 & 1151 & 1152 \\
0.1 & 1035 & 1051 & 1053 & 1044 & 1054 & 1047 & 1047 \\
0.2 & 947 & 963 & 965 & 957 & 966 & 960 & 960 \\
0.3 & 871 & 889 & 891 & 883 & 891 & 886 & 886 \\
0.4 & 803 & 826 & 827 & 819 & 827 & 822 & 823 \\
0.4999 & 696 & 771 & 772 & 764 & 772 & 768 & 768 \\
\hline
\end{tabular}

Table 8: Volumetric strain energy $U_{\text {vol }}$ for Example 3.3 (3D analysis, $h_{1}=10, h_{2}=10$, $h_{3}=10, a=4 \times 10^{-6}$ ).

\begin{tabular}{|c|c|c|c|c|c|c|c|}
\hline$\nu$ & CST4 & USH8 & UST8 & EUST9 & UST10 & EUST11 & exact \\
\hline 0.0 & 3.20 & 0.19 & 1.53 & 0.53 & 1.56 & $8 \mathrm{e}-4$ & 0 \\
0.1 & 3.81 & 0.14 & 1.18 & 0.56 & 1.34 & $8 \mathrm{e}-4$ & 0 \\
0.2 & 4.74 & 0.09 & 0.86 & 0.58 & 1.10 & $7 \mathrm{e}-4$ & 0 \\
0.3 & 6.32 & 0.06 & 0.57 & 0.58 & 0.81 & $6 \mathrm{e}-4$ & 0 \\
0.4 & 9.76 & 0.03 & 0.28 & 0.50 & 0.46 & $5 \mathrm{e}-4$ & 0 \\
0.4999 & 0.32 & $2 \mathrm{e}-5$ & $3 \mathrm{e}-4$ & $1 \mathrm{e}-3$ & $5 \mathrm{e}-4$ & $8 \mathrm{e}-7$ & 0 \\
\hline
\end{tabular}




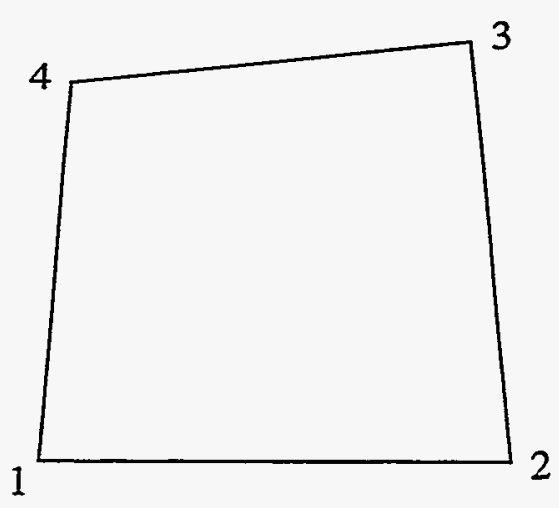

- (a)

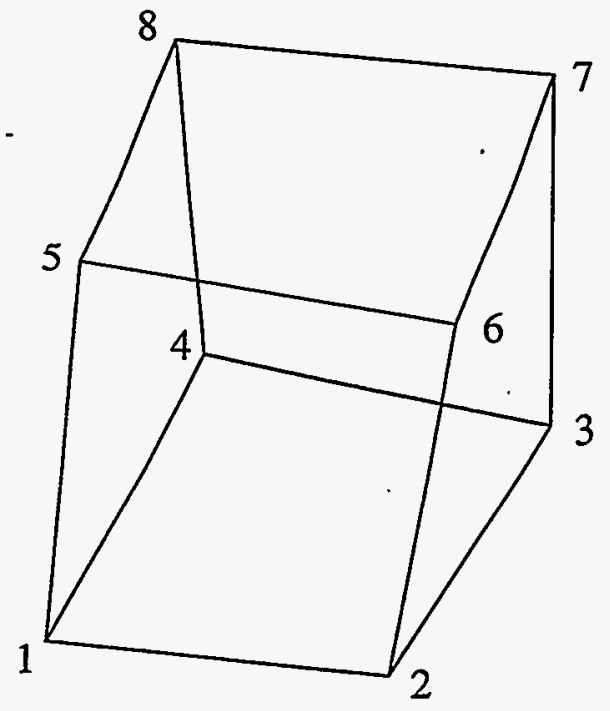

(b)

Figure 1: Geometry and node numbering of uniform strain quadrilateral and hexahedron. 


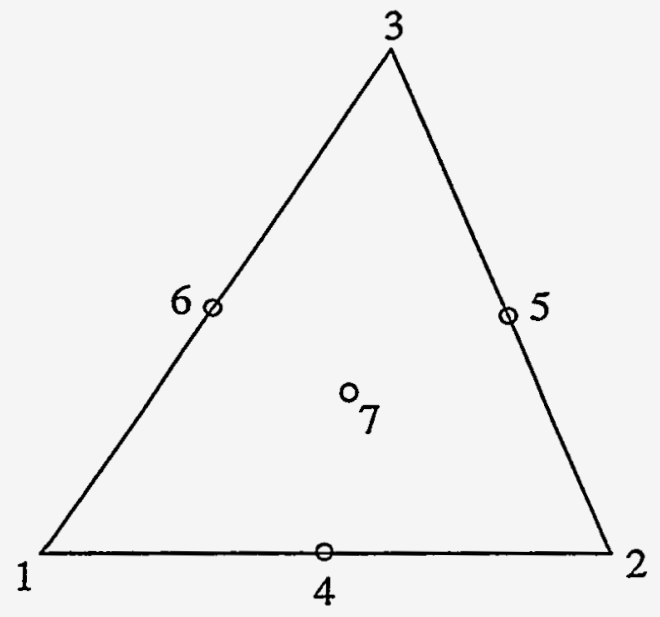

(a)

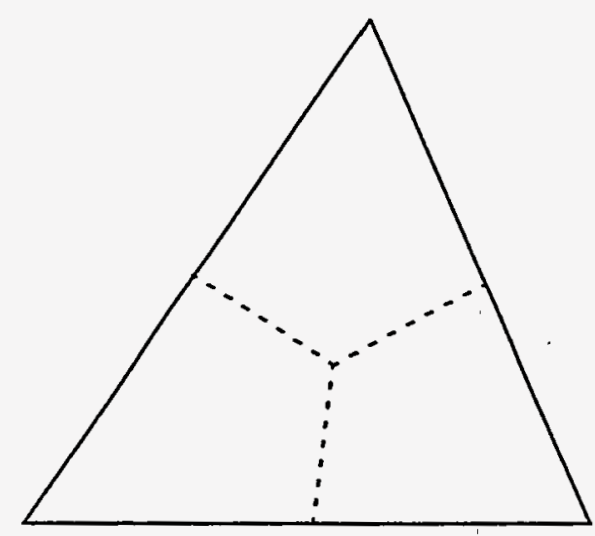

(b)

Figure 2: Seven-node enhanced uniform strain triangle (EUST7). 


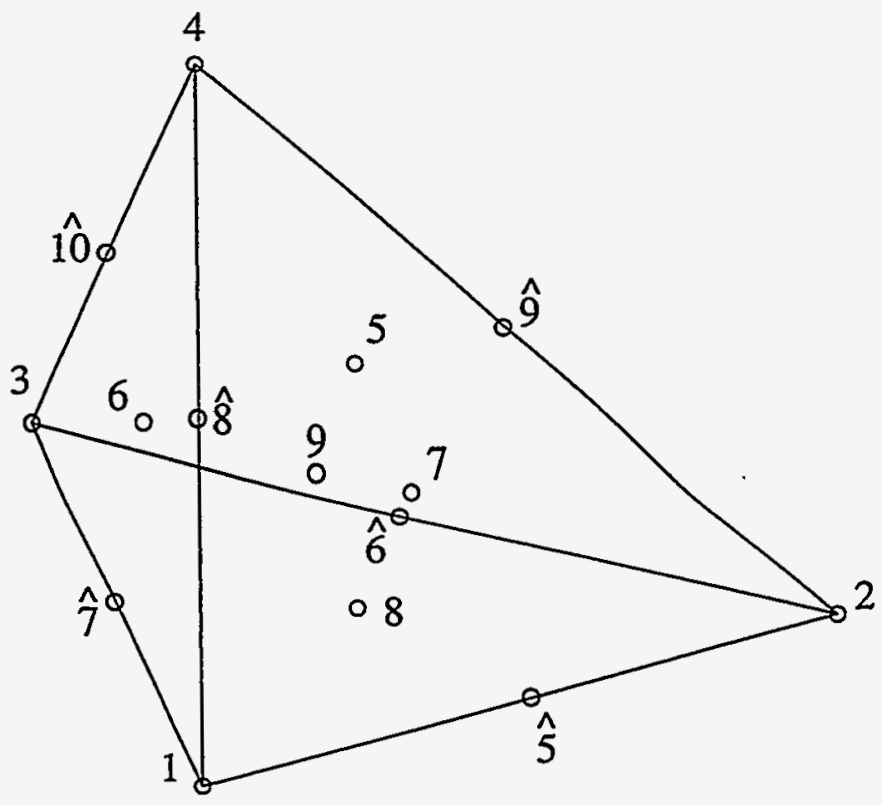

(a)

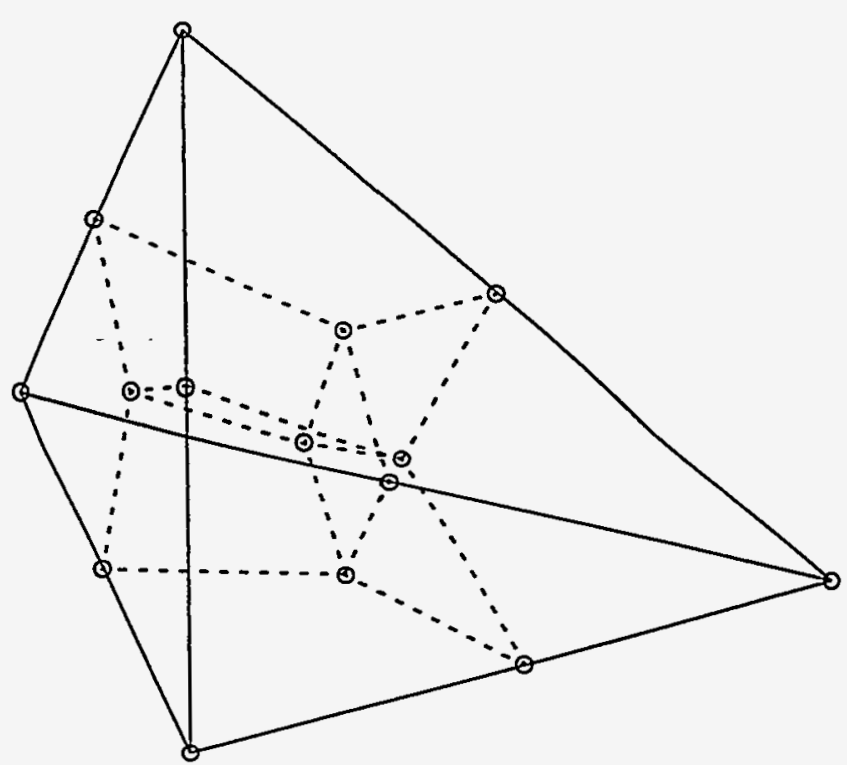

(b)

Figure 3: Nine-node enhanced uniform strain tetrahedron (EUST9). 


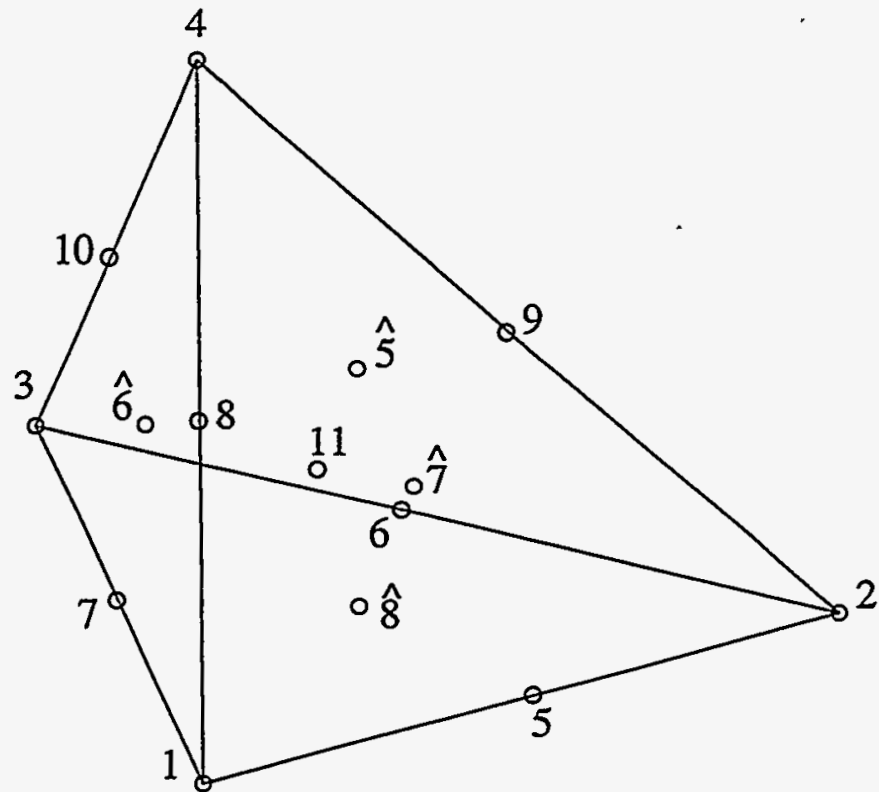

(a)

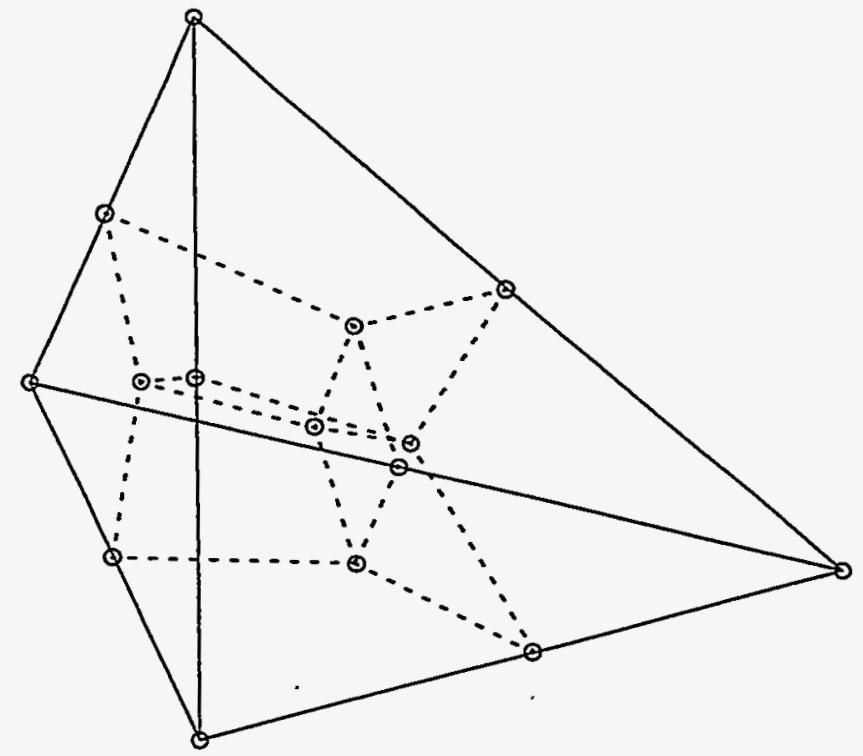

(b)

Figure 4: Eleven-node enhanced uniform strain tetrahedron (EUST11). 


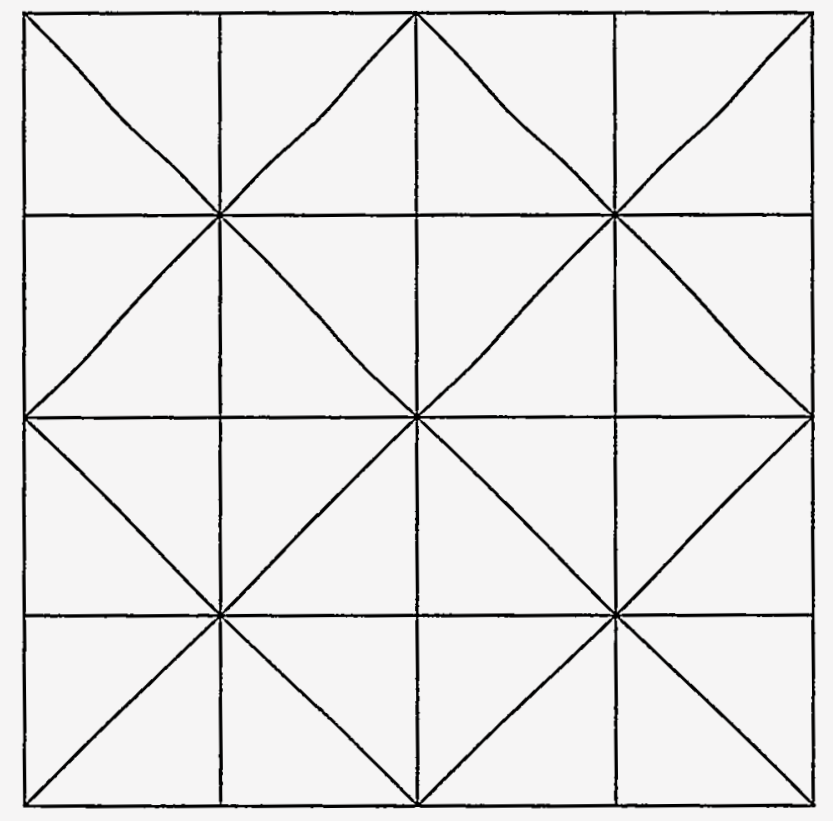

(a)

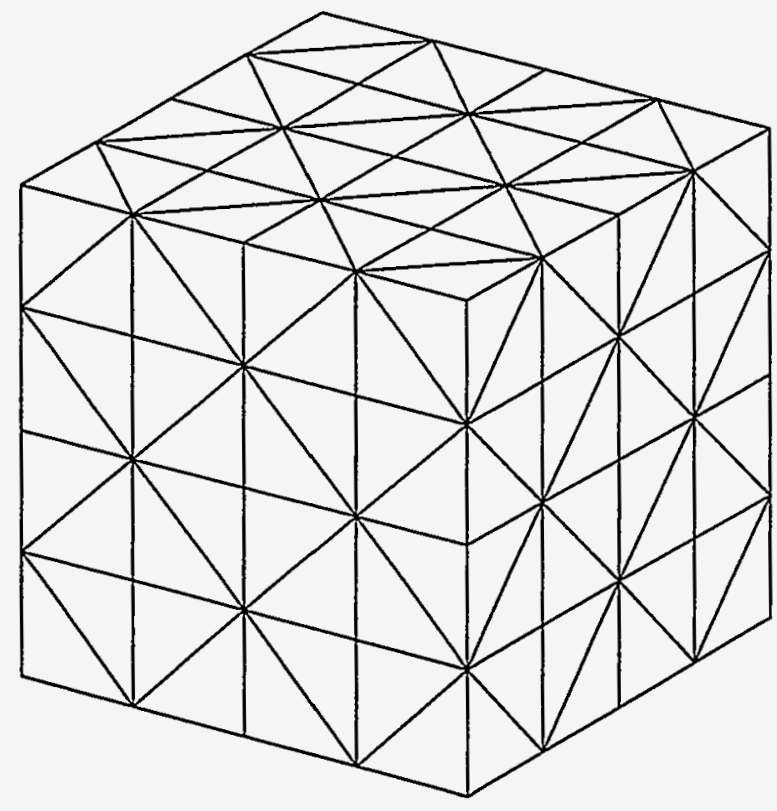

(c)

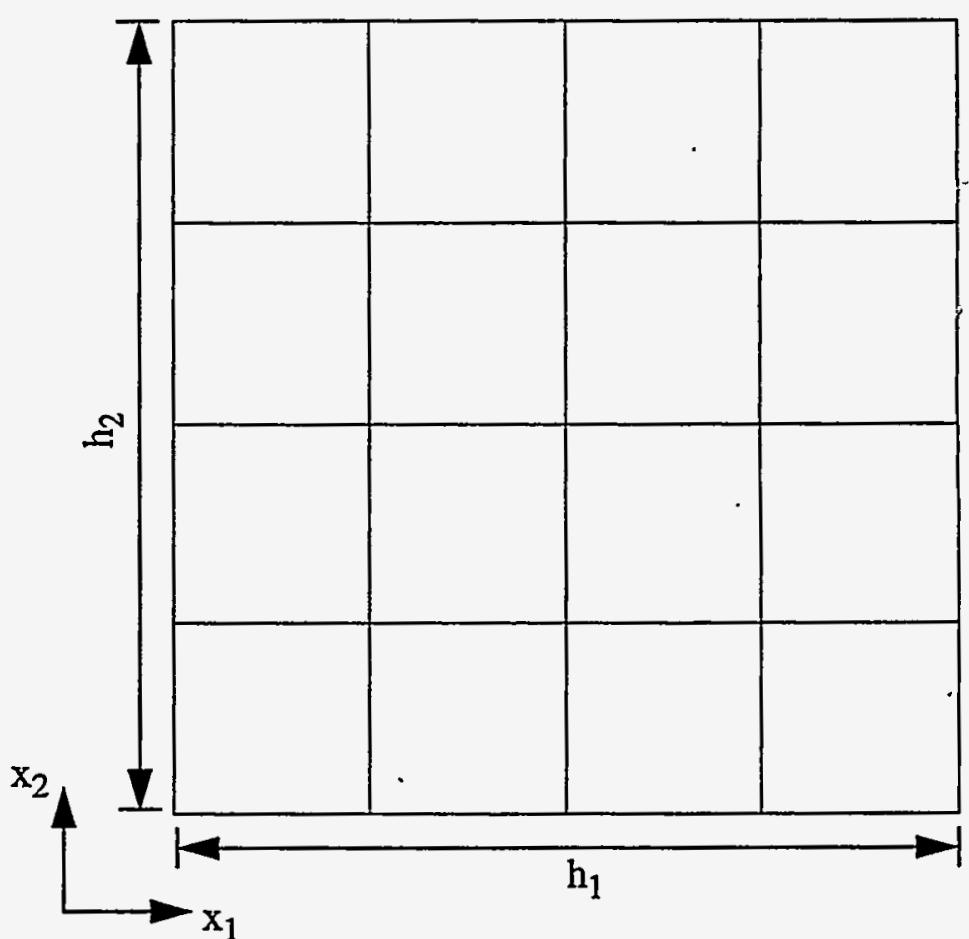

(b)

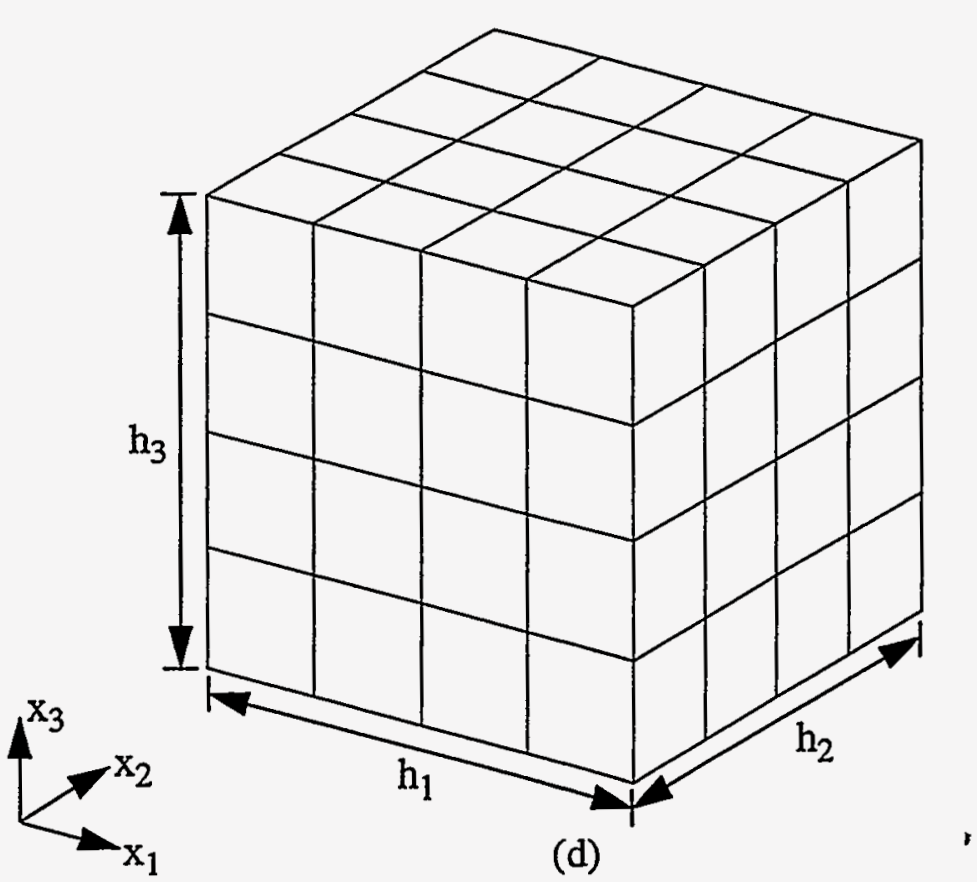

Figure 5: Triangular (a), quadrilateral (b), tetrahedral (c), and hexahedral (d) meshes used in the example problems for $n=4$. 


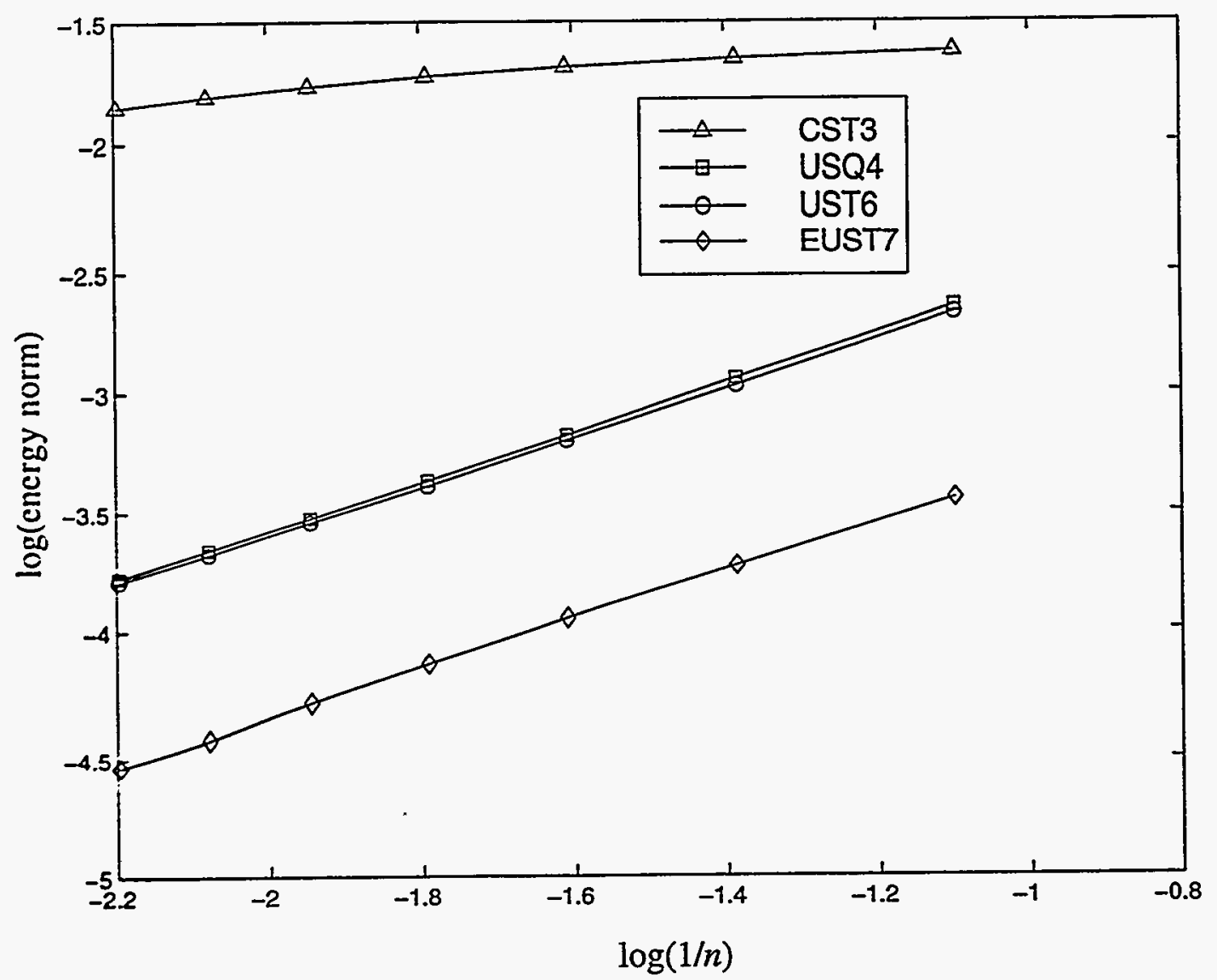

Figure 6: Energy norms of 2D plane stress elements for Example 3.1 with $h_{1}=10, h_{2}=1$, $R=10^{4}$ and $v=0.3$. The slopes near unity of the lines are characteristic of linear displacement/constant stress elements. 


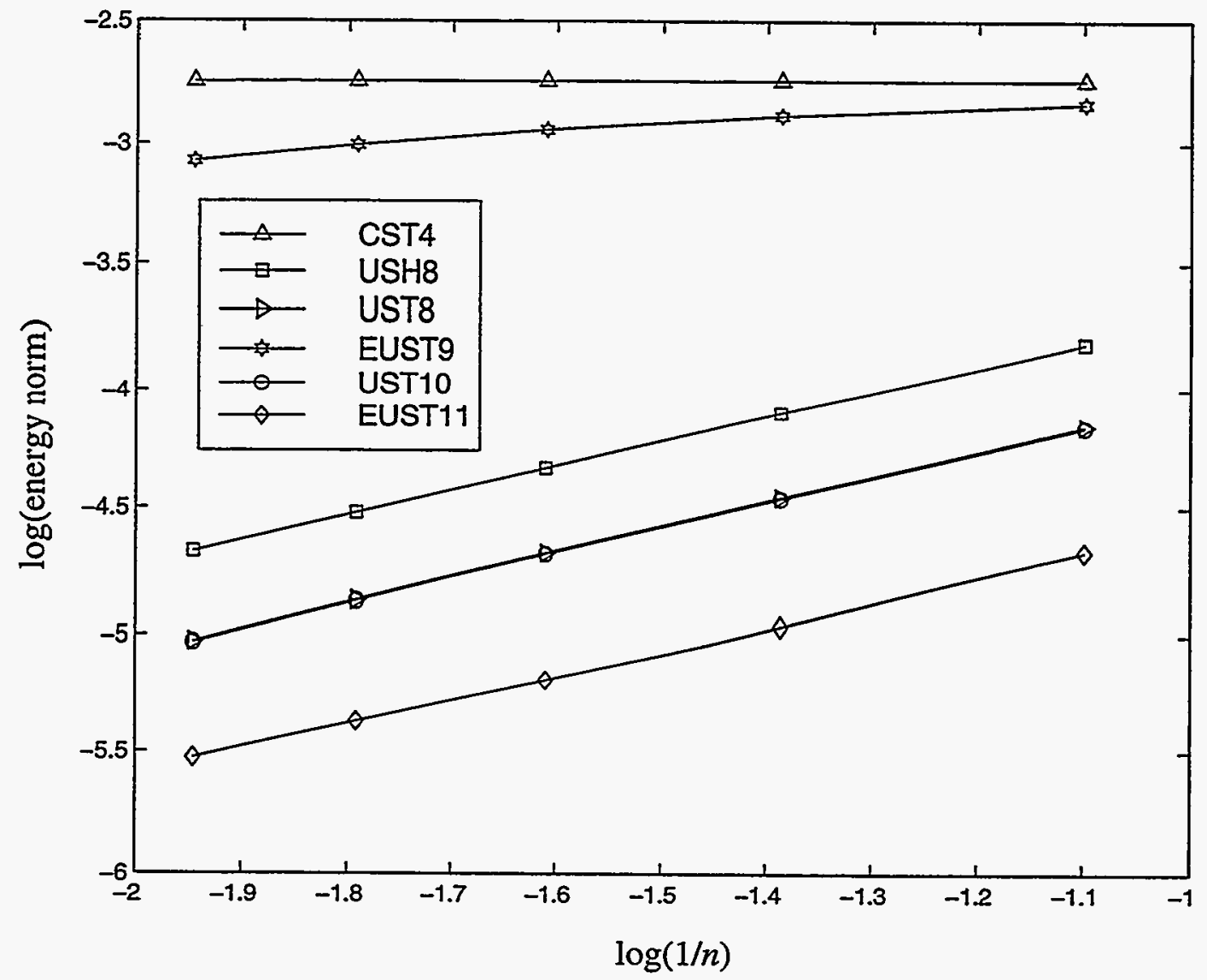

Figure 7: Energy norms of 3D elements for Example 3.1 with $h_{1}=10, h_{2}=1, h_{3}=0.1$ and $R=10^{4}$ and $v=0.3$. 


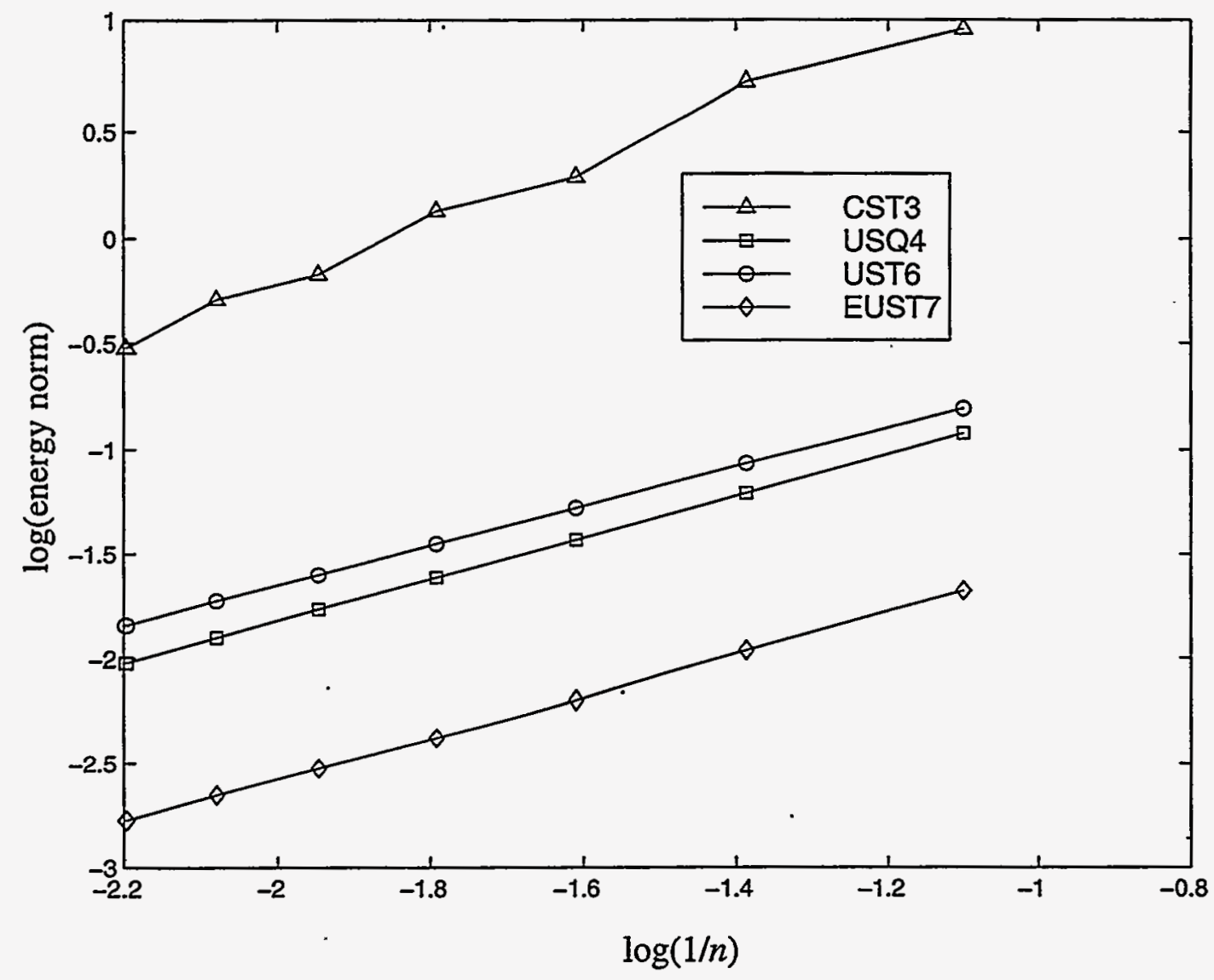

Figure 8: Energy norms of 2D plane strain elements for Example 3.2 with $h_{1}=10, h_{2}=10$, $a=4 \times 10^{-6}$ and $v=0.499$. 


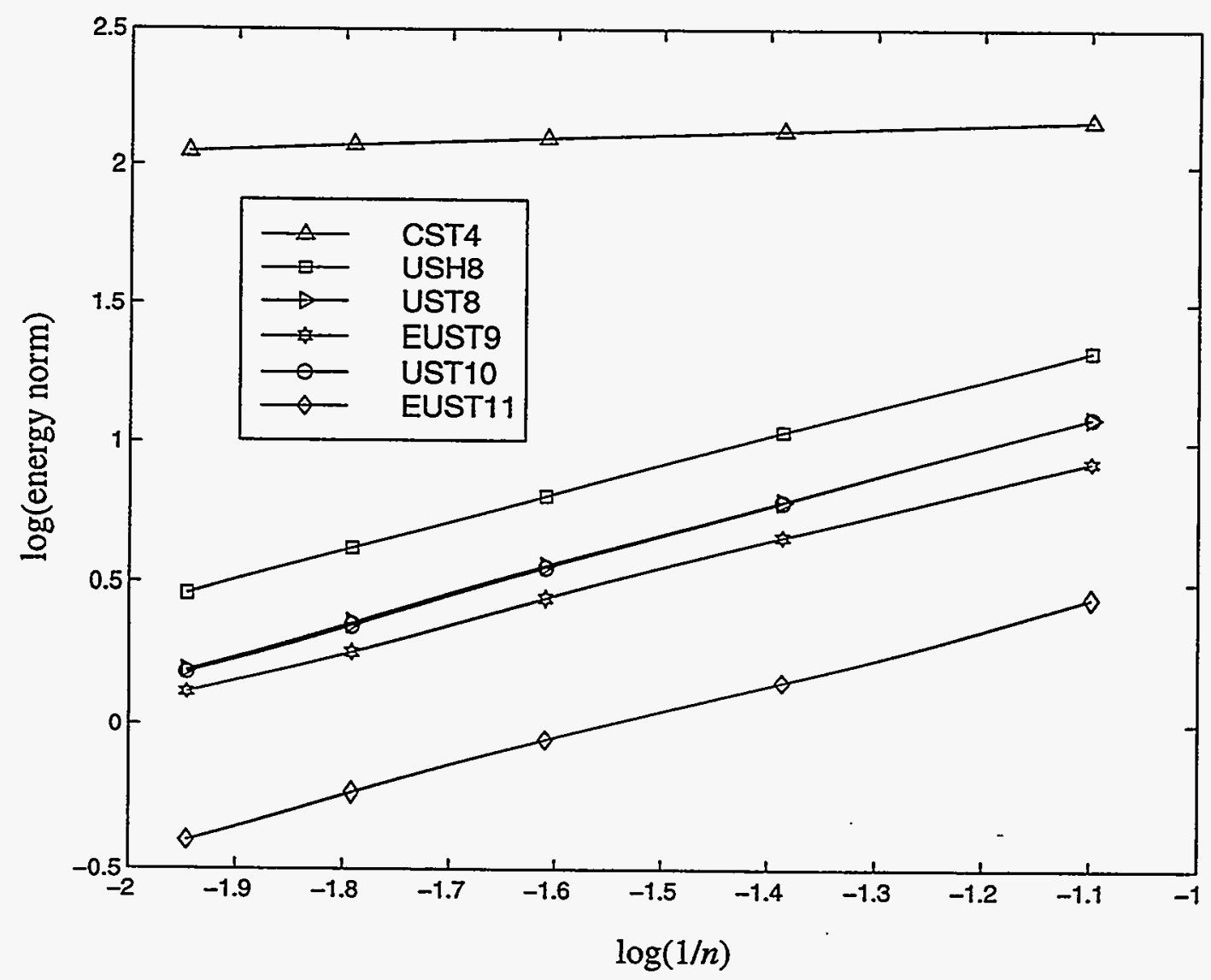

Figure 9: Energy norms of 3D elements for Example 3.3 with $h_{1}=10, h_{2}=10, h_{3}=10$, $a=4 \times 10^{-6}$ and $v=0.499$. 


\title{
A Transition Element for Uniform Strain Hexahedral and Tetrahedral Finite Elements ${ }^{1}$
}

\author{
C. R. Dohrmann ${ }^{2}$ \\ S. W. $\mathrm{Key}^{3}$
}

\begin{abstract}
A transition element is presented for meshes containing uniform strain hexahedral and tetrahedral finite elements. It is shown that the volume of the standard uniform strain hexahedron is identical to that of a polyhedron with fourteen vertices and twenty four triangular faces. Based on this equivalence, a transition element is developed as a simple modification of the uniform strain hexahedron. The transition element makes use of a general method for hourglass control and satisfies first-order patch tests. Example problems in linear elasticity are included to demonstrate the application of the element.
\end{abstract}

Key Words. Finite elements, transition element, uniform strain, hourglass control.

\footnotetext{
${ }^{1}$ Sandia is a multiprogram laboratory operated by Sandia Corporation, a Lockheed Martin Company, for the United States Department of Energy under Contract DE-AL04-94AL8500.

${ }^{2}$ Structural Dynamics Department, Sandia National Laboratories, MS 0439, Albuquerque, New Mexico 87185-0439, email: crdohrm@sandia.gov, phone: (505) 844-8058, fax: (505) 844-9297.

${ }^{3}$ Engineering and Manufacturing Mechanics Department, Sandia National Laboratories, MS 0443, Albuquerque, New Mexico 87185-0443.
} 


\section{Introduction}

The uniform strain hexahedron [1] is a standard element used in several commercial and research-based finite element codes. Much of the popularity of this element can be attributed to its inherent simplicity and computational efficiency. While the element is well suited to a variety of problems in explicit transient dynamics and statics, its practical use depends on the ability to generate high-quality, all-hexahedral meshes in reasonable amounts of time. This capability exists for many problems, but for others the mesh generation process may be very difficult and require unreasonable amounts of time.

The unavailability of a robust, automated, all-hexahedral mesher motivated recent investigations of a family of uniform strain tetrahedral elements [2-3]. These elements were shown to possess the same convergence and anti-locking characteristics of the uniform strain hexahedron. An important advantage of the tetrahedron over the hexahedron is its ability to more readily mesh complicated geometries. On the other hand, more tetrahedral elements are generally required to mesh a volume for a specified element edge length.

The purpose of this study is to investigate a transition element for meshes containing both uniform strain hexahedral and tetrahedral elements. This effort is motivated by the idea of meshing a geometry primarily with hexahedral elements. For regions of the mesh that cannot be completed with hexahedral elements, a direct transition to tetrahedral elements could be made to complete the mesh. In this way, the advantages of both element types could be brought to bear on the meshing problem.

One approach to connect hexahedral and tetrahedral elements involves the use of pyramid elements [4]. Given a face of a hexahedron adjacent to two tetrahedra, methods are available for inserting a pyramid element between the two element types. The pyramid provides a conforming transition, but its insertion may degrade the quality of surrounding elements. The present approach does not require the use of pyramid elements.

The basis for the transition element is the equivalence of the volume of the uniform strain hexahedron and a polyhedron with fourteen vertices and twenty four triangular faces. Eight of the vertices are the nodes of the hexahedron. The remaining six vertices are located at the geometric centers of the six faces of the hexahedron. Based on this equivalence, a transition element is obtained as a simple modification of the uniform strain hexahedron.

The element formulations for the uniform strain hexahedron, transition element, and uniform strain tetrahedron are presented in the following section. A method for hourglass control that is applicable to all three element types is also presented. The third section includes example problems in linear elasticity. The final section discusses applications to higher-order elements and connecting dissimilar finite element meshes at a shared boundary.

\section{Element Formulations}

Element formulations for the uniform strain hexahedron, transition element, and the uniform strain tetrahedron are presented in this section. These formulations are based on the uniform strain approach of Reference 1. A general method for hourglass control is also presented. 
Given a uniform strain element with volume $V$, the so-called $B$ matrix is defined as

$$
B_{i I}=\frac{\partial V}{\partial x_{i I}}
$$

where $x_{i I}(i=1,2,3$ and $I=1, \ldots, N)$ are the coordinates of node $I$. Following the development in Ref. 1 , the nodal forces associated with the element stresses are given by

$$
f_{i I}=\sum_{j=1}^{3} \bar{T}_{i j} B_{j I}
$$

where $\bar{T}_{i j}$ are elements of the Cauchy stress tensor (assumed constant throughout the element).

All the elements discussed in this section use the same formulation. The only differences are the volume expressions and the number of nodes per element. The uniform strain hexahedron and the transition element each have eight nodes. The uniform strain tetrahedron has four vertex nodes and from one to four mid-face nodes.

\subsection{Uniform Strain Hexahedron}

Consider a hexahedral element with nodal coordinates $\left(x_{1 I}, x_{2 I}, x_{3 I}\right)$ for $I=1, \ldots, 8$. Spatial coordinates coordinates $x_{1}, x_{2}$, and $x_{3}$ are related to isoparametric coordinates $\eta_{1}$, $\eta_{2}$ and $\eta_{3}$ by the equations

$$
x_{i}\left(\eta_{1}, \eta_{2}, \eta_{3}\right)=\sum_{I=1}^{8} x_{i I} \phi_{I}\left(\eta_{1}, \eta_{2}, \eta_{3}\right)
$$

where

$$
\begin{array}{ll}
\phi_{1}=\left(1-\eta_{1}\right)\left(1-\eta_{2}\right)\left(1-\eta_{3}\right) / 8 & \phi_{2}=\left(1+\eta_{1}\right)\left(1-\eta_{2}\right)\left(1-\eta_{3}\right) / 8 \\
\phi_{3}=\left(1+\dot{\eta}_{1}\right)\left(1+\eta_{2}\right)\left(1-\eta_{3}\right) / 8 & \phi_{4}=\left(1-\eta_{1}\right)\left(1+\eta_{2}\right)\left(1-\eta_{3}\right) / 8 \\
\phi_{5}=\left(1-\eta_{1}\right)\left(1-\eta_{2}\right)\left(1+\eta_{3}\right) / 8 & \phi_{6}=\left(1+\eta_{1}\right)\left(1-\eta_{2}\right)\left(1+\eta_{3}\right) / 8 \\
\phi_{7}=\left(1+\eta_{1}\right)\left(1+\eta_{2}\right)\left(1+\eta_{3}\right) / 8 & \phi_{8}=\left(1-\eta_{1}\right)\left(1+\eta_{2}\right)\left(1+\eta_{3}\right) / 8
\end{array}
$$

The Jacobian determinant $J$ of the element is given by

$$
J=\operatorname{det}\left[\begin{array}{lll}
\frac{\partial x_{1}}{\partial \eta_{1}} & \frac{\partial x_{2}}{\partial \eta_{1}} & \frac{\partial x_{3}}{\partial \eta_{1}} \\
\frac{\partial x_{1}}{\partial \eta_{2}} & \frac{\partial x_{2}}{\partial \eta_{2}} & \frac{\partial x_{3}}{\partial \eta_{2}} \\
\frac{\partial x_{1}}{\partial \eta_{3}} & \frac{\partial x_{2}}{\partial \eta_{3}} & \frac{\partial x_{3}}{\partial \eta_{3}}
\end{array}\right]
$$

The volume of the hexahedron can be expressed in terms of $J$ by

$$
V^{\text {hex }}=\int_{-1}^{1} \int_{-1}^{1} \int_{-1}^{1} J d \eta_{1} d \eta_{2} d \eta_{3}
$$


The $B$ matrix of the hexahedron is defined as

$$
B_{i I}^{h e x}=\frac{\partial V^{h e x}}{\partial x_{i I}}
$$

Equations for $B_{i I}^{\text {hex }}$ are provided in Reference 1. In addition, one has

$$
V^{h e x}=\sum_{I=1}^{8} x_{1 I} B_{1 I}^{h e x}=\sum_{I=1}^{8} x_{2 I} B_{2 I}^{h e x}=\sum_{I=1}^{8} x_{3 I} B_{3 I}^{h e x}
$$

\subsection{Transition Element}

Consider a polyhedron with fourteen vertices and twenty four triangular faces. Eight of the vertices are the nodes of the hexahedron. The remaining vertices are located at the geometric centers of the six faces of the hexahedron. The coordinates of these vertices are given by

$$
\begin{array}{ll}
x_{i a}=\left(x_{i 1}+x_{i 2}+x_{i 6}+x_{i 5}\right) / 4 & x_{i b}=\left(x_{i 3}+x_{i 4}+x_{i 8}+x_{i 7}\right) / 4 \\
x_{i c}=\left(x_{i 5}+x_{i 6}+x_{i 7}+x_{i 8}\right) / 4 & x_{i d}=\left(x_{i 2}+x_{i 1}+x_{i 4}+x_{i 3}\right) / 4 \\
x_{i e}=\left(x_{i 4}+x_{i 1}+x_{i 5}+x_{i 8}\right) / 4 & x_{i f}=\left(x_{i 2}+x_{i 3}+x_{i 7}+x_{i 6}\right) / 4
\end{array}
$$

The triangular faces of the polyhedron are given by the following twenty four nodal 3-tuples: 12a, 26a. 65a. 51a. 34b, 48b, 87b, 73b, 56c, 67c, 78c, 85c, 21d, 14d, 43d, 32d, 41e, 15e, 58e, $84 e, 23 f .37 f .76 f$. and $62 f$. Twelve of the triangular faces are shown in Figure 1.

The volume of the polyhedron can be calculated by decomposing it into a collection of twenty four tetrahedra:

$$
\begin{aligned}
V^{p}= & V_{g 12 a}+V_{g 26 a}+V_{g 65 a}+V_{g 51 a}+V_{g 34 b}+V_{g 48 b}+V_{g 87 b}+V_{g 73 b}+ \\
& V_{g 56 c}+V_{g 67 c}+V_{g 78 c}+V_{g 85 c}+V_{g 21 d}+V_{g 14 d}+V_{g 43 d}+V_{g 32 d}+ \\
& V_{g 41 e}+V_{g 15 e}+V_{g 58 e}+V_{g 84 e}+V_{g 23 f}+V_{g 37 f}+V_{g 76 f}+V_{g 62 f}
\end{aligned}
$$

where

$$
x_{i g}=\sum_{I=1}^{8} x_{i I} / 8
$$

is a "center" node and

$$
\begin{aligned}
V_{I J K L}= & {\left[\left(x_{1 J}-x_{1 I}\right)\left(x_{2 K} x_{3 L}-x_{2 L} x_{3 K}\right)+\left(x_{1 I}-x_{1 K}\right)\left(x_{2 J} x_{3 L}-x_{2 L} x_{3 J}\right)+\right.} \\
& \left(x_{1 L}-x_{1 I}\right)\left(x_{2 J} x_{3 K}-x_{2 K} x_{3 J}\right)+\left(x_{1 K}-x_{1 J}\right)\left(x_{2 I} x_{3 L}-x_{2 L} x_{3 I}\right)+ \\
& \left.\left(x_{1 J}-x_{1 L}\right)\left(x_{2 I} x_{3 K}-x_{2 K} x_{3 I}\right)+\left(x_{1 L}-x_{1 K}\right)\left(x_{2 I} x_{3 J}-x_{2 J} x_{3 I}\right)\right] / 6
\end{aligned}
$$

With the aid of symbolic mathematical software [5], one can show that the expressions for $V^{h e x}$ and $V^{p}$ are identical (see Eqs. 9 and 15). Consequently, for purposes of calculating the $B$ matrix, one can consider the element boundary of the hexahedron to be that of the polyhedron described above. 
For the moment, assume that all of the faces of the transition element are attached to uniform strain hexahedral elements except for face 2143. To this face are attached two uniform strain tetrahedral elements. The triangular faces of the attached tetrahedral elements are 143 and 321 (see Figure 2).

In order for the transition element to conform to the two triangular faces of the tetrahedral elements, its connecting face must be modified. The connecting face of the transition element originally has the four triangular faces $21 d, 14 d, 43 d$ and $32 d$. The modified connecting face is defined to have triangular faces $210,140,430$ and 320 where

$$
x_{i 0}=\left(x_{i 1}+x_{i 3}\right) / 2
$$

Notice that faces 140 and 430 combine to form face 143 and faces 210 and 320 combine to form face 321 . Accordingly, the volume of the transition element is given by

$$
V^{t e l}=V^{h e x}-\hat{V}
$$

where the volume mismatch $\hat{V}$ between the standard hexahedron and the polyhedron with the modified connecting face is given by the following sum of four tetrahedral subvolumes:

$$
\hat{V}=V_{140 d}+V_{430 d}+V_{320 d}+V_{210 d}
$$

The elements of the $B$ matrix of the transition element are defined as

$$
B_{i I}^{t e l}=\frac{\partial V^{t e l}}{\partial x_{i I}}
$$

Substituting Eqs. (19-20) and (10) into Eq. (21) and using the chain rule for differentiation (see Eqs. 13b,18), one obtains

$$
\begin{aligned}
B_{i 1}^{\text {tel }} & =B_{i 1}^{\text {hex }}-\frac{\partial \hat{V}}{\partial x_{i 1}}-\frac{1}{4} \frac{\partial \hat{V}}{\partial x_{i d}}-\frac{1}{2} \frac{\partial \hat{V}}{\partial x_{i 0}} \\
B_{i 2}^{\text {tel }} & =B_{i 2}^{\text {hex }}-\frac{\partial \hat{V}}{\partial x_{i 2}}-\frac{1}{4} \frac{\partial \hat{V}}{\partial x_{i d}} \\
B_{i 3}^{\text {tel }} & =B_{i 3}^{\text {hex }}-\frac{\partial \hat{V}}{\partial x_{i 3}}-\frac{1}{4} \frac{\partial \hat{V}}{\partial x_{i d}}-\frac{1}{2} \frac{\partial \hat{V}}{\partial x_{i 0}} \\
B_{i 4}^{\text {tel }} & =B_{i 4}^{\text {hex }}-\frac{\partial \hat{V}}{\partial x_{i 4}}-\frac{1}{4} \frac{\partial \hat{V}}{\partial x_{i d}} \\
B_{i I}^{\text {tel }} & =B_{i I}^{\text {hex }} \text { for } I=5, \ldots, 8
\end{aligned}
$$

The derivatives appearing in Eqs. (22-25) can be calculated using the equations

$$
\begin{aligned}
& \partial V_{I J K L} / \partial x_{1 I}=\left[\left(x_{2 K}-x_{2 L}\right)\left(x_{3 J}-x_{3 L}\right)-\left(x_{2 J}-x_{2 L}\right)\left(x_{3 K}-x_{3 L}\right)\right] / 6 \\
& \partial V_{I J K L} / \partial x_{2 I}=\left[\left(x_{3 K}-x_{3 L}\right)\left(x_{1 J}-x_{1 L}\right)-\left(x_{3 J}-x_{3 L}\right)\left(x_{1 K}-x_{1 L}\right)\right] / 6 \\
& \partial V_{I J K L} / \partial x_{3 I}=\left[\left(x_{1 K}-x_{1 L}\right)\left(x_{2 J}-x_{2 L}\right)-\left(x_{1 J}-x_{1 L}\right)\left(x_{2 K}-x_{2 L}\right)\right] / 6
\end{aligned}
$$


A similar procedure for calculating $B_{i I}^{\text {tel }}$ can be used for all other possible connections to tetrahedral elements. In addition, the corrections given by Eqs. (22-25) can be repeated for all faces of the hexahedral transition element connected to tetrahedral elements. The programming for this process simply loops over all the faces in the transition element that are connected to tetrahedral elements.

Although not investigated in the present study, an alternative transition element can be derived by removing the constraint in Eq. (13b). The connecting face of the transition element would remain defined by the four triangular faces $21 d, 14 d, 43 d$ and $32 d$, but node $d$ would no longer be dependent on the other four nodes of the face. Thus, the face of the alternative transition element would be connected to four rather than two tetrahedral elements.

\subsection{Uniform Strain Tetrahedron}

Consider the uniform strain tetrahedron [2] shown in Figure 3. The element shown has four mid-face nodes, but we consider a family of elements with from one to four mid-face nodes. For the purposes here, a face of a tetrahedral element connected to a transition element does not have a mid-face node.

The uniform strain tetrahedron used in this study corresponds to the element with $\alpha=$ $1 / 3$ in Reference 2 . The volume of the tetrahedral element is given by

$$
V^{\text {tet }}=V_{1234}+\beta_{5} V_{2345}+\beta_{6} V_{1436}+\beta_{7} V_{1247}+\beta_{8} V_{1328}
$$

where $\beta_{j}=1$ if mid-face node $j$ exists and $\beta_{j}=0$ if mid-face node $j$ is not present. The elements of the $B$ matrix for the tetrahedral element are given by

$$
B_{i I}^{t e t}=\frac{\partial V^{t e t}}{\partial x_{i I}}
$$

\subsection{Hourglass Control}

A general method for hourglass control is presented here. The method was developed previously in Reference 2 and is applicable to all the element types considered in this study. Hourglass control is included to remove spurious zero energy modes from an element. We presently only consider hourglass stiffness, but one could easily include hourglass damping for problems in dynamics.

Let $u_{i I}$ denote the nodal displacements of an element and define

$$
d_{i}=\left[\begin{array}{llll}
u_{i 1} & u_{i 2} & \ldots & u_{i N}
\end{array}\right]^{T}
$$

The vector $d_{i}$ can be expressed as

$$
d_{i}=\Phi q+\Phi_{\perp} q_{\perp}
$$


where

$$
\Phi=\left[\begin{array}{cccc}
1 & \bar{x}_{11} & \bar{x}_{21} & \bar{x}_{31} \\
1 & \bar{x}_{12} & \bar{x}_{22} & \bar{x}_{32} \\
\vdots & \vdots & \vdots & \vdots \\
1 & \bar{x}_{1 N} & \bar{x}_{2 N} & \bar{x}_{3 N}
\end{array}\right]
$$

and

$$
\bar{x}_{i I}=x_{i I}-x_{i g}
$$

The term $x_{i g}$ is given by Eq. (16) with the number 8 replaced by $N$. Premultiplying Eq. (33) by $\Phi^{T}$ and solving for $q$ yields

$$
q=\left(\Phi^{T} \Phi\right)^{-1} \Phi^{T} d_{i}
$$

Substituting Eq. (37) into Eq. (33) leads to

$$
\Phi_{\perp} q_{\perp}=\left[I-\Phi\left(\Phi^{T} \Phi\right)^{-1} \Phi^{T}\right] d_{i}
$$

The strain energy associated with hourglass stiffness is formulated as

$$
U_{h}=\epsilon V^{1 / 3} G_{h}\left(\Phi_{\perp} q_{\perp}\right)^{T}\left(\Phi_{\perp} q_{\perp}\right) / 2
$$

where $\epsilon$ is a positive scalar and $G_{h}$ is a material modulus. Substituting Eq. (38) into Eq. (39) leads to

$$
U_{h}=\epsilon V^{1 / 3} G_{h} d_{i}^{T}\left[I-\Phi\left(\Phi^{T} \Phi\right)^{-1} \Phi^{T}\right] d_{i} / 2
$$

Finally, the nodal force vector $f_{i h}$ associated with hourglass stiffness is obtained by differentiating $U_{h}$ with respect to $d_{i}$. The result is

$$
f_{i h}=\epsilon V^{1 / 3} G_{h}\left[I-\Phi\left(\Phi^{T} \Phi\right)^{-1} \Phi^{T}\right] d_{i}
$$

It follows from Eq. (41) that $f_{i h}$ is orthogonal to $\Phi q$. In other words, hourglass stiffness does not cause any restoring forces if the nodal displacements are consistent with a linear displacement field, the desired result.

Carrying out the mathematical operations in Eq. (41), the elements of $f_{i h}$ can be expressed on a nodal basis as

$$
f_{i h I}=\epsilon V^{1 / 3} G_{h}\left[u_{i I}-\frac{1}{N} \sum_{I=1}^{N} u_{i I}-\bar{x}_{1 I} \sum_{I=1}^{N} a_{1 I} u_{i I}-\bar{x}_{2 I} \sum_{I=1}^{N} a_{2 I} u_{i I}-\bar{x}_{3 I} \sum_{I=1}^{N} a_{3 I} u_{i I}\right]
$$

where

$$
\begin{aligned}
& a_{1 I}=c_{1} \bar{x}_{1 I}+c_{4} \bar{x}_{2 I}+c_{6} \bar{x}_{3 I} \\
& a_{2 I}=c_{2} \bar{x}_{2 I}+c_{5} \bar{x}_{3 I}+c_{4} \bar{x}_{1 I} \\
& a_{3 I}=c_{3} \bar{x}_{3 I}+c_{6} \bar{x}_{1 I}+c_{5} \bar{x}_{2 I}
\end{aligned}
$$




$$
\begin{gathered}
c_{0}=s_{x x} s_{y y} s_{z z}+2 s_{x y} s_{y z} s_{z x}-s_{x x} s_{y z}^{2}-s_{y y} s_{z x}^{2}-s_{z z} s_{x y}^{2} \\
c_{1}=\left(s_{y y} s_{z z}-s_{y z}^{2}\right) / c_{0}, \quad c_{4}=\left(s_{y z} s_{z x}-s_{x y} s_{z z}\right) / c_{0} \\
c_{2}=\left(s_{z z} s_{x x}-s_{z x}^{2}\right) / c_{0}, \quad c_{5}=\left(s_{z x} s_{x y}-s_{y z} s_{x x}\right) / c_{0} \\
c_{3}=\left(s_{x x} s_{y y}-s_{x y}^{2}\right) / c_{0}, \quad c_{6}=\left(s_{x y} s_{y z}-s_{z x} s_{y y}\right) / c_{0}
\end{gathered}
$$

and

$$
\begin{array}{ll}
s_{x x}=\sum_{I=1}^{N} \bar{x}_{1 I} \bar{x}_{1 I}, \quad s_{y y}=\sum_{I=1}^{N} \bar{x}_{2 I} \bar{x}_{2 I}, \quad s_{z z}=\sum_{I=1}^{N} \bar{x}_{3 I} \bar{x}_{3 I} \\
s_{x y}=\sum_{I=1}^{N} \bar{x}_{1 I} \bar{x}_{2 I}, \quad s_{y z}=\sum_{I=1}^{N} \bar{x}_{2 I} \bar{x}_{3 I}, \quad s_{z x}=\sum_{I=1}^{N} \bar{x}_{3 I} \bar{x}_{1 I}
\end{array}
$$

The same method of hourglass control can be used for all three element types. Meshes containing the elements presented in Sections 2.1-2.3 pass first-order patch tests exactly.

\section{Example Problems}

All the example problems in this section assume small deformations of a linear, elastic, isotropic material with Young's modulus $E$ and Poisson's ratio $\nu$. Let,

$$
d=\left[\begin{array}{llllllllll}
u_{11} & u_{21} & u_{31} & u_{12} & u_{22} & u_{32} & \ldots & u_{1 N} & u_{2 N} & u_{3 N}
\end{array}\right]^{T}
$$

Corresponding to $d$ are stiffness matrices associated with elastic strains and hourglass control. With reference to Eq. (2), the stiffness matrix for elastic strains is expressed as

$$
K^{e s}=\hat{B}^{T} H \hat{B} / V
$$

where

$$
\begin{gathered}
H=\left[\begin{array}{cccccc}
2 G+\lambda & \lambda & \lambda & 0 & 0 & 0 \\
\lambda & 2 G+\lambda & \lambda & 0 & 0 & 0 \\
\lambda & \lambda & 2 G+\lambda & 0 & 0 & 0 \\
0 & 0 & 0 & G & 0 & 0 \\
0 & 0 & 0 & 0 & G & 0 \\
0 & 0 & 0 & 0 & 0 & G
\end{array}\right] \\
G=\frac{E}{2(1+\nu)} \\
\lambda=\frac{E \nu}{(1+\nu)(1-2 \nu)}
\end{gathered}
$$

The nonzero elements of the matrix $\hat{B}$ are given by

$$
\begin{array}{lll}
\hat{B}_{1,3(I-1)+1}=B_{1 I} & \hat{B}_{2,3(I-1)+2}=B_{2 I} & \hat{B}_{3,3(I-1)+3}=B_{3 I} \\
\hat{B}_{4,3(I-1)+1}=B_{2 I} & \hat{B}_{5,3(I-1)+2}=B_{3 I} & \hat{B}_{6,3(I-1)+3}=B_{1 I}
\end{array}
$$




$$
\hat{B}_{4,3(I-1)+2}=B_{1 I} \quad \hat{B}_{5,3(I-1)+3}=B_{2 I} \quad \hat{B}_{6,3(I-1)+1}=B_{3 I}
$$

With reference to Eq. (42), the nonzero elements of the stiffness matrix for hourglass control are given by

$$
K_{3(I-1)+i, 3(J-1)+i}^{h g}=\epsilon V^{1 / 3} G_{h}\left(\delta_{I J}-1 / N-\bar{x}_{1 I} a_{1 J}-\bar{x}_{2 I} a_{2 J}-\bar{x}_{3 I} a_{3 J}\right)
$$

where $\delta_{I J}$ is the Kronecker delta. The stiffness matrix of the element is the sum of $K^{e s}$ and $K^{\text {hg }}$. That is,

$$
K=K^{e s}+K^{h g}
$$

Element stiffness matrices are assembled as is done conventionally to form the stiffness matrix of the entire model. All the example problems are for a material with $E=10^{7}$ using hourglass control specified by $\epsilon=0.05$ and $G_{h}=G$.

Consider a cube that is meshed uniformly with $n^{3}$ hexahedral elements where $n$ is the number of elements per edge. In this study, attention is restricted to values of $n$ that are multiples of three. Mixed-element meshes are obtained by replacing the $(n / 3)^{3}$ hexahedral elements in the center of the mesh with $(n / 3)^{3}$ blocks of tetrahedral elements. Each of these blocks is made up of five tetrahedral elements. The mixed-element meshes and all-hexahedral meshes are designated by $n m$ and $n h$, respectively. A view of mesh $6 m$ with several of the hexahedral and transition elements removed is shown in Figure 4. The mesh contains a combination of hexahedral, tetrahedral and transition elements. The outer boundaries of the meshes are defined by $x_{i}=0$ and $x_{i}=10$ for $i=1,2,3$.

\subsection{Example 1}

The first example deals with the classic problem of pure bending. The applied tractions on the face defined by $x_{1}=10$ are given by

$$
\sigma_{11}\left(x_{2}, x_{3}\right)=E\left(5-x_{2}\right) / R
$$

The displacement boundary conditions for the problem are specified as

$$
\begin{array}{r}
u_{1}\left(0, x_{2}, x_{3}\right)=0 \\
u_{2}(0,0,0)=0 \\
u_{3}(0,0,0)=0 \\
u_{2}(0,0,10)=0
\end{array}
$$

The elasticity solution to the boundary value problem is given by

$$
\begin{aligned}
& u_{1}\left(x_{1}, x_{2}, x_{3}\right)=x_{1}\left(5-x_{2}\right) / R \\
& u_{2}\left(x_{1}, x_{2}, x_{3}\right)=\frac{1}{2 R}\left[x_{1}^{2}+\nu\left[\left(5-x_{2}\right)^{2}-\left(5-x_{3}\right)^{2}\right]\right] \\
& u_{3}\left(x_{1}, x_{2}, x_{3}\right)=\nu\left(x_{2} x_{3}-5 x_{2}-5 x_{3}\right) / R
\end{aligned}
$$


The deviatoric and volumetric strain energies for the problem are given by

$$
\begin{aligned}
& E_{\text {dev }}=\frac{25000 E(1+\nu)}{9 R^{2}} \\
& E_{\text {vol }}=\frac{12500 E(1-2 \nu)}{9 R^{2}}
\end{aligned}
$$

All the results presented for this example are for $R=10^{4}$.

Calculated values of the volumetric and deviatoric strain energies for meshes $3 m, 6 m$, $9 m$ and $9 h$ are shown in Table 1 . Meshes $3 m, 6 m$ and $9 m$ contain a combination of uniform strain hexahedral, tetrahedral, and transition elements. Notice that the results for meshes $9 m$ and $9 h$ are nearly identical. In addition, none of the meshes suffer from volumetric locking for values of $\nu$ near 0.5 .

Plots of the energy norm of the error (see Ref. 6) for the the mixed-element and allhexahedral meshes are shown in Figure 5 for $\nu=0.3$. The same information is presented in Figure 6 for $\nu=0.4999$. Notice that there is very little difference in the accuracy and convergence characteristics of the two mesh types. The slopes near unity of the lines in the figures are consistent with the convergence rate of linear displacement/constant stress elements.

\subsection{Example 2}

The first part of this example considers the problem of specifying the displacements of all nodes on the boundaries $x_{i}=0$ and $x_{i}=10(i=1,2,3)$ as follows:

$$
\begin{aligned}
& u_{1}\left(x_{1}, x_{2}, x_{3}\right)=a\left(x_{2}^{2}+x_{3}^{2}-2 x_{1}^{2}+2 x_{1} x_{2}+2 x_{1} x_{3}+5 x_{2} x_{3}\right) \\
& u_{2}\left(x_{1}, x_{2}, x_{3}\right)=a\left(x_{3}^{2}+x_{1}^{2}-2 x_{2}^{2}+2 x_{2} x_{3}+2 x_{2} x_{1}+5 x_{3} x_{1}\right) \\
& u_{3}\left(x_{1}, x_{2}, x_{3}\right)=a\left(x_{1}^{2}+x_{2}^{2}-2 x_{3}^{2}+2 x_{3} x_{1}+2 x_{3} x_{2}+5 x_{1} x_{2}\right)
\end{aligned}
$$

The elasticity solution to the associated boundary value problem is also given by Eqs. (72-74). The deviatoric strain energy is given by

$$
E_{\text {dev }}=144 G a^{2}(10)^{5}
$$

One can confirm that the elasticity solution has no volumetric strain. That is,

$$
\frac{\partial u_{1}}{\partial x_{1}}+\frac{\partial u_{2}}{\partial x_{2}}+\frac{\partial u_{3}}{\partial x_{3}}=0
$$

Consequently, the exact value of the volumetric strain energy $E_{v o l}$ is zero. All the results presented for this example are for $a=4 \times 10^{-6}$.

Calculated values of the volumetric and deviatoric strain energies for meshes $3 m, 6 m$, $9 m$ and $9 h$ are shown in Table 2. Notice that the results for meshes $9 m$ and $9 h$ are nearly identical. The volumetric strain energies are nearly zero for all meshes except $3 \mathrm{~m}$. For the all-hexahedral mesh $9 h$, the volumetric strain energy is identically zero for all values of $\nu$. This remarkable result only holds if none of the hexahedral elements are skewed. 
The primary reason for the volumetric locking of mesh $3 m$ for values of $\nu$ near 0.5 is a shortage of unconstrained nodes to accommodate zero volume change of all the elements. In the first example, no volumetric locking occurred for mesh $3 m$ because a much smaller number of displacement boundary conditions were imposed. This fact shows that the volumetric locking of a mesh can be caused by the boundary conditions and may not be a characteristic of the mesh itself. The all-hexahedral mesh does not suffer from volumetric locking only because of the regular arrangement of nodes on a grid.

Plots of the energy norm of the error are shown are shown in Figure 7 for $\nu=0.3$. The same information is presented in Figure 8 for $\nu=0.4999$. Notice that there is very little difference in the asymptotic accuracy and convergence of the two mesh types. The only distinct difference is for mesh $3 m$ with $\nu=0.4999$.

Rather than prescribing the displacements of all nodes on the boundary, alternative boundary conditions are considered in which the stresses corresponding to the elasticity solution (see Eqs. 72-74) are applied to the outer boundary of the mesh. Rigid body motion is restricted by the displacement boundary conditions

$$
\begin{array}{rrl}
u_{1}(0,0,0)=0 & u_{2}(0,0,0)=0 & u_{3}(0,0,0)=0 \\
u_{1}(0,10,0)=100 a & u_{2}(0,0,10)=100 a & u_{3}(10,0,0)=100 a
\end{array}
$$

The energy norm of the error is plotted in Figure 9 for $\nu=0.4999$. Notice from the figure that the differences in results for the mixed-element meshes and all-hexahedral meshes are negligible.

\subsection{Example 3}

The final example is used to show that volumetric locking can also occur for all-hexahedral meshes if the boundary conditions are overly stringent. To illustrate this point, consider meshes $3 m$ and $3 h$ with the following modifications. The coordinates of eight internal nodes are modified as follows:

$$
\begin{array}{ll}
\left(c_{1}, c_{1}, c_{1}\right) & \left(c_{1}+0.5, c_{1}+0.5, c_{1}\right) \\
\left(c_{2}, c_{1}, c_{1}\right) & \left(c_{2}+0.2, c_{1}+0.6, c_{1}\right) \\
\left(c_{2}, c_{2}, c_{1}\right) & \left(c_{2}-0.3, c_{2}-0.3, c_{1}\right) \\
\left(c_{1}, c_{2}, c_{1}\right) & \left(c_{1}-0.4, c_{2}-0.6, c_{1}\right) \\
\left(c_{1}, c_{1}, c_{2}\right) & \rightarrow\left(c_{1}+0.7, c_{1}, c_{2}\right) \\
\left(c_{2}, c_{1}, c_{2}\right) & \left(c_{2}+0.3, c_{1}, c_{2}\right) \\
\left(c_{2}, c_{2}, c_{2}\right) & \left(c_{2}+0.5, c_{2}, c_{2}\right) \\
\left(c_{1}, c_{2}, c_{2}\right) & \left(c_{1}-0.3, c_{2}, c_{2}\right)
\end{array}
$$

where $c_{1}=3+1 / 3$ and $c_{2}=6+2 / 3$. The locations of the mid-face nodes of the tetrahedral elements in mesh $3 m$ are based on the eight modified nodal coordinates.

Values of $E_{d e v}$ and $E_{v o l}$ for the two meshes are shown in Table 3 for the same displacement boundary conditions used in Example 2. As a result of moving the internal nodes, the allhexahedral mesh actually suffers more from volumetric locking than the mixed-element mesh 
for values of $\nu$ near 0.5 . The extent of locking for either mesh depends entirely on the modified nodal coordinates. Both meshes clearly lock as $\nu$ approaches 0.5 . The point of the example is simply to show that all-hexahedral meshes can also suffer from volumetric locking for problems with overly stringent boundary conditions.

Although the example problems here are restricted to linear elasticity, we expect the performance of mixed meshes of uniform strain hexahedra and tetrahedra to be similar to that of all-hexahedra or all-tetrahedra meshes for problems with geometric or material nonlinearities (see Ref. 3). For such problems, the method of hourglass control presented in Section 2.4 can be expressed in rate form and remains applicable to all three element types. Since finite deformations lead to self similar geometric configurations, the constructions introduced here for properly matching hexahedral to tetrahedral elements remain the same. Of course, for each new mesh distortion, a reevaluation of the correction terms is required.

\section{Other Applications}

What has been demonstrated here is a method for mixing hexahedral and tetrahedral elements in a single mesh that are based on the uniform strain concepts of Reference 1. That is, interface terms have been derived that allow a mixed mesh of particular hexahedral and tetrahedral finite elements to satisfy first-order Irons patch tests and to converge for second-order Irons patch tests under mesh refinement.

Given that the underlying shape functions can reproduce exactly a linear displacement field, the uniform strain approach not only preserves linear consistency in the constant-stress gradient/divergence operator $B_{i I}$ used here, but it explicitly provides (describes) the linearconsistency kernel that any gradient/divergence operator designed to capture more complex element behavior must contain.

Since a first-order Irons patch test is a test of the ability of an arbitrary collection of finite elements to reproduce a constant strain field, it is a test for linear consistency, and, therefore, a test for the presence of a linearly consistent constant-stress kernel in the finite element's construction.

One may conclude that hexahedra and tetrahedra of any order may be used together in a mixed mesh provided (1) the interface volume (plus or minus) between the respective element types can be explicitly constructed and (2) corrections to the gradient/divergence operator of one class or the other of the bounding elements are constructed using uniform strain concepts as was done in Equations 22-26.

The approach here does not, however, serve to ensure quadratic displacement (linear strain) continuity. Thus, in the presence of linear strain gradients, one can expect no more than linear strain convergence at the interface between quadratic or higher displacement elements matched together by the approach here.

The transition element was derived by replacing a face of a hexahedron with the triangular faces of two adjacent tetrahedra. In other words, part of the boundary definition of the hexahedron was replaced by the boundary definition of adjacent elements. The same basic idea has been used successfully to connect dissimilar finite element meshes at a shared boundary [7]. The connected meshes pass first-order patch tests and yield superior results 
to those obtained using existing methods based on master-slave concepts for connecting two meshes.

\section{Conclusions}

A transition element for meshes containing uniform strain hexahedral and tetrahedral elements is presented. Meshes containing the transition element satisfy first-order patch tests and converge for second-order patch tests under mesh refinement. Comparisons with allhexahedral meshes show that mixed-element meshes do not cause any significant degradation in accuracy, convergence rates or locking behavior for a variety of problems. Guidelines are established for extending the present approach to higher-order elements and for connecting dissimilar finite element meshes at a shared boundary.

\section{References}

1. D. P. Flanagan and T. Belytschko, 'A Uniform Strain Hexahedron and Quadrilateral with Orthogonal Hourglass Control', International Journal for Numerical Methods in Engineering, 17, 679-706 (1981).

2. C. R. Dohrmann, S. W. Key, M. W. Heinstein and J. Jung, 'A Least Squares Approach for Uniform Strain Triangular and Tetrahedral Finite Elements', International Journal for Numerical Methods in Engineering, 42, 1181-1197 (1998).

3. S. W. Key, M. W. Heinstein, C. M. Stone, F. J. Mello, M. L. Blanford and K. G. Budge, 'A Suitable Low-Order, 8-Node Tetrahedral Finite Element for Solids', to appear in International Journal for Numerical Methods in Engineering.

4. S. J. Owen, S. A. Canann and S. Saigal, 'Pyramid Elements for Maintaining Tetrahedra to Hexahedra Conformability', Trends in Unstructured Mesh Generation, AMDVol. 220 ASME, 123-129 (1997).

5. K. M. Heal, M. L. Hansen and K. M. Rickard, Maple V Learning Guide, Springer, New York, New York, 1996.

6. O. C. Zienkiewicz and R. L. Taylor, The Finite Element Method, Vol. 1, 4th Ed., McGraw-Hill, New York, New York, 1989.

7. C. R. Dohrmann, S. W. Key and M. W. Heinstein, 'A Method for Connecting Dissimilar Finite Element Meshes in Two Dimensions', submitted to International Journal for Numerical methods in Engineering. 
Table 1: Example 1 strain energies for meshes $3 \dot{m}, 6 m, 9 m$ and $9 h$.

\begin{tabular}{|c|c|c|c|c|c|c|c|c|c|c|}
\hline$\nu$ & \multicolumn{2}{|c|}{$3 m$} & \multicolumn{2}{|c|}{$6 m$} & \multicolumn{2}{|c|}{$9 m$} & \multicolumn{2}{|c|}{$9 h$} & \multicolumn{2}{|c|}{ exact } \\
\hline & $E_{\text {dev }}$ & $E_{\text {vol }}$ & $E_{\text {dev }}$ & $E_{\text {vol }}$ & $E_{\text {dev }}$ & $E_{\text {vol }}$ & $E_{\text {dev }}$ & $E_{\text {vol }}$ & $E_{\text {dev }}$ & $E_{\text {vol }}$ \\
\hline 0.0 & 302 & 150 & 283 & 142 & 280 & 140 & 280 & 140 & 278 & 139 \\
0.1 & 332 & 121 & 312 & 113 & 308 & 112 & 308 & 112 & 306 & 111 \\
0.2 & 362 & 90.9 & 340 & 85.2 & 336 & 84.2 & 336 & 84.2 & 333 & 83.3 \\
0.3 & 393 & 60.9 & 369 & 56.9 & 364 & 56.1 & 364 & 56.1 & 361 & 55.6 \\
0.4 & 423 & 30.6 & 397 & 28.5 & 393 & 28.1 & 393 & 28.1 & 389 & 27.8 \\
0.499 & 454 & 0.308 & 425 & 0.285 & 420 & 0.281 & 420 & 0.281 & 416 & 0.27 \\
0.4999 & 454 & $3.07 \mathrm{e}-2$ & 426 & $2.85 \mathrm{e}-2$ & 421 & $2.81 \mathrm{e}-2$ & 421 & $2.81 \mathrm{e}-2$ & 417 & $2.78 \mathrm{e}-2$ \\
0.49999 & 454 & $3.07 \mathrm{e}-3$ & 426 & $2.85 \mathrm{e}-3$ & 421 & $2.81 \mathrm{e}-3$ & 421 & $2.81 \mathrm{e}-3$ & 417 & $2.78 \mathrm{e}-3$ \\
\hline
\end{tabular}

Table 2: Example 2 strain energies for meshes $3 m, 6 m, 9 m$ and $9 h$.

\begin{tabular}{|c|c|c|c|c|c|c|c|c|c|}
\hline$\nu$ & \multicolumn{2}{|c|}{$3 m$} & \multicolumn{2}{c|}{$6 m$} & \multicolumn{2}{c|}{$9 m$} & \multicolumn{2}{|c|}{$9 h$} & exact \\
\hline & $E_{\text {dev }}$ & $E_{\text {vol }}$ & $E_{\text {dev }}$ & $E_{\text {vol }}$ & $E_{\text {dev }}$ & $E_{\text {vol }}$ & $E_{\text {dev }}$ & $E_{\text {vol }}$ & $E_{\text {dev }}$ \\
\hline 0.0 & 1132 & 0.09 & 1147 & 0.02 & 1150 & 0.01 & 1150 & 0 & 1152 \\
0.1 & 1029 & 0.09 & 1043 & 0.02 & 1045 & 0.01 & 1045 & 0 & 1047 \\
0.2 & 944 & 0.09 & 956 & 0.02 & 958 & 0.01 & 958 & 0 & 960 \\
0.3 & 871 & 0.11 & 882 & 0.02 & 884 & 0.01 & 884 & 0 & 886 \\
0.4 & 809 & 0.17 & 819 & 0.01 & 821 & 0.003 & 821 & 0 & 823 \\
0.499 & 755 & 14 & 765 & 0.04 & 767 & 0.02 & 767 & 0 & 769 \\
0.4999 & 755 & $1.4 \mathrm{e} 2$ & 765 & 0.18 & 767 & 0.06 & 767 & 0 & 768 \\
0.49999 & 755 & $1.4 \mathrm{e} 3$ & 765 & 0.10 & 767 & 0.03 & 767 & 0 & 768 \\
\hline
\end{tabular}

Table 3: Example 3 strain energies for meshes $3 m$ and $3 h$.

\begin{tabular}{|c|c|c|c|c|}
\hline$\nu$ & \multicolumn{2}{|c|}{$3 m$} & \multicolumn{2}{c|}{$3 h$} \\
\hline & $E_{\text {dev }}$ & $E_{\text {vol }}$ & $E_{\text {dev }}$ & $E_{\text {vol }}$ \\
\hline 0.0 & 1132 & 0.0835 & 1132 & 0.0174 \\
0.1 & 1029 & 0.0908 & 1029 & 0.0208 \\
0.2 & 944 & 0.105 & 943 & 0.0262 \\
0.3 & 871 & 0.136 & 871 & 0.0359 \\
0.4 & 809 & 0.232 & 809 & 0.0621 \\
0.499 & 760 & 4.62 & 756 & 3.28 \\
0.4999 & 769 & 1.83 & 759 & 21.4 \\
0.49999 & 772 & 2.13 & 794 & 117 \\
0.499999 & 773 & 16.2 & 863 & 854 \\
\hline
\end{tabular}




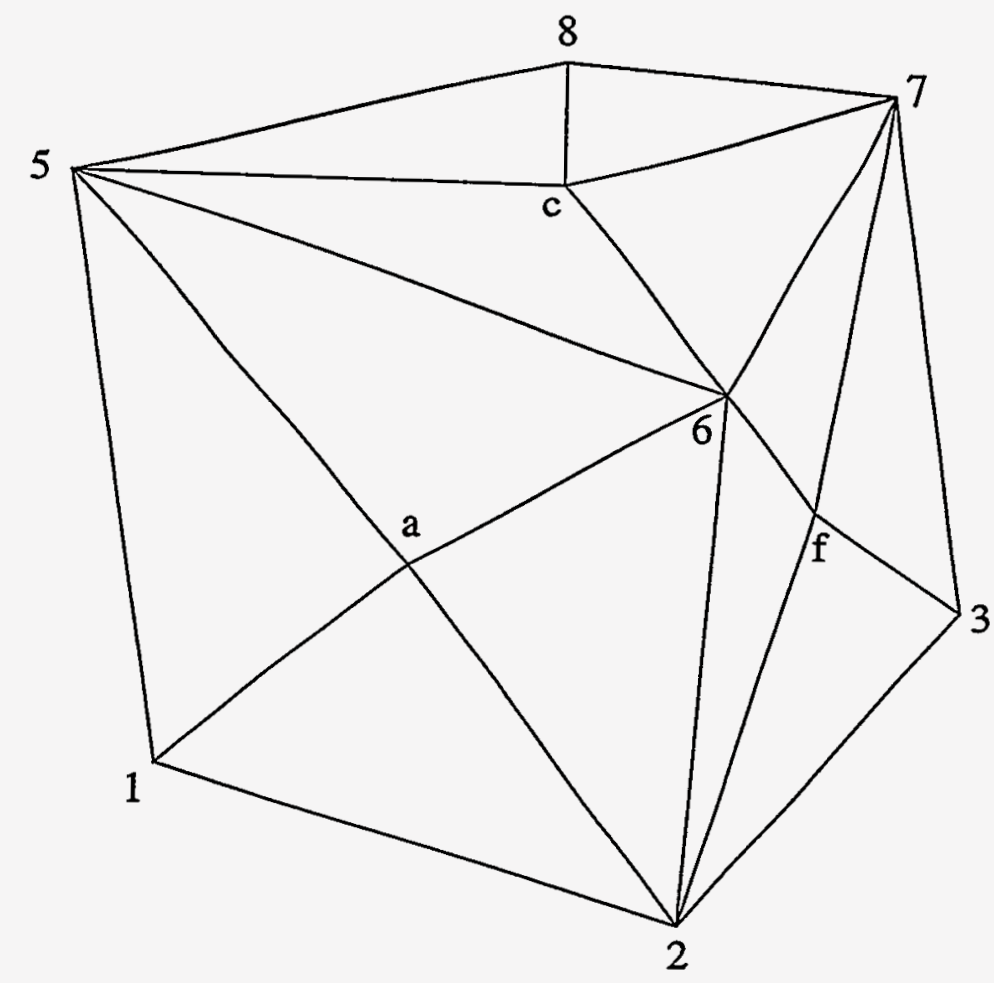

Figure 1: Sketch of polyhedron showing 12 of the 24 triangular faces. 


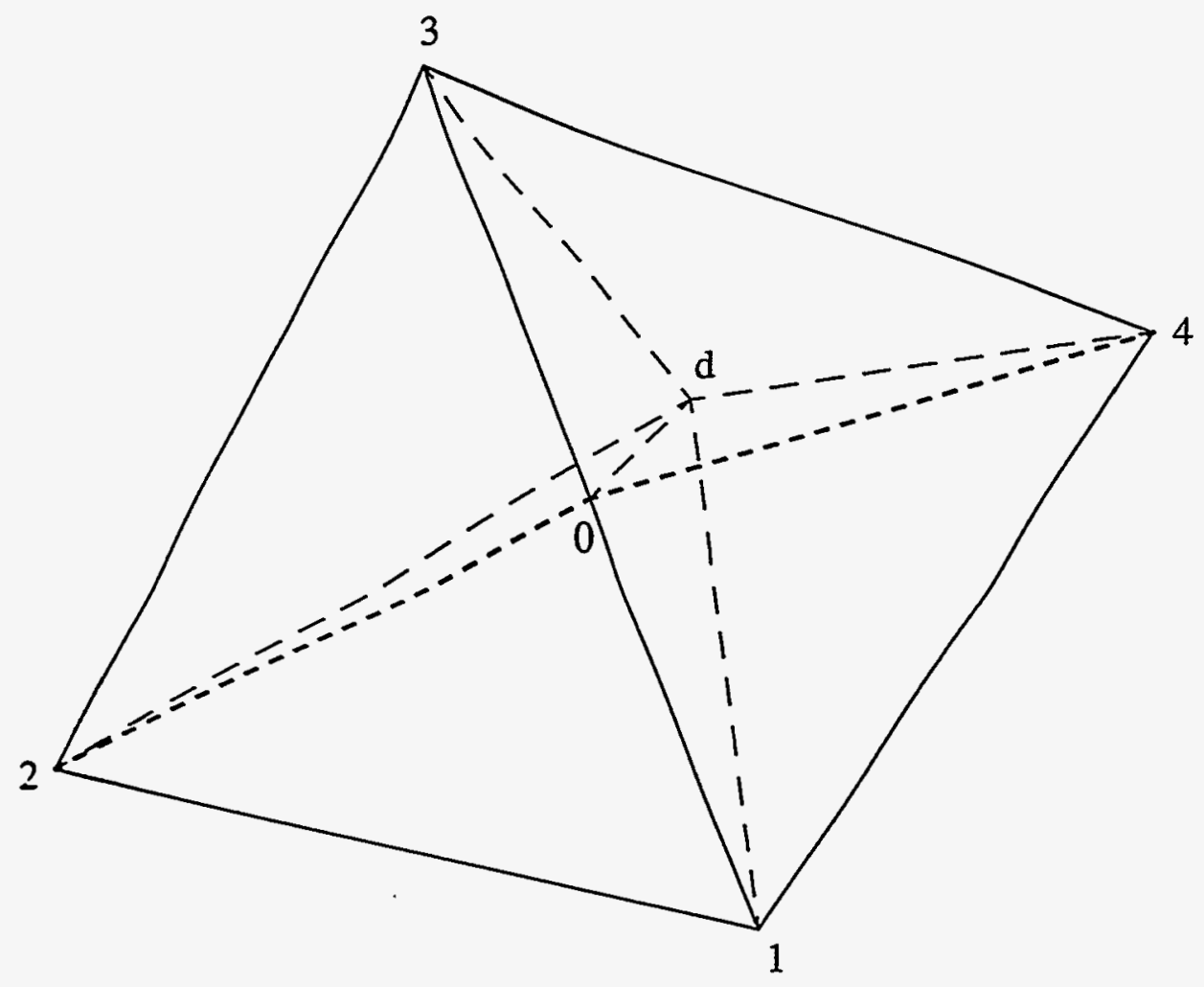

Figure 2: Connecting face of transition element. 


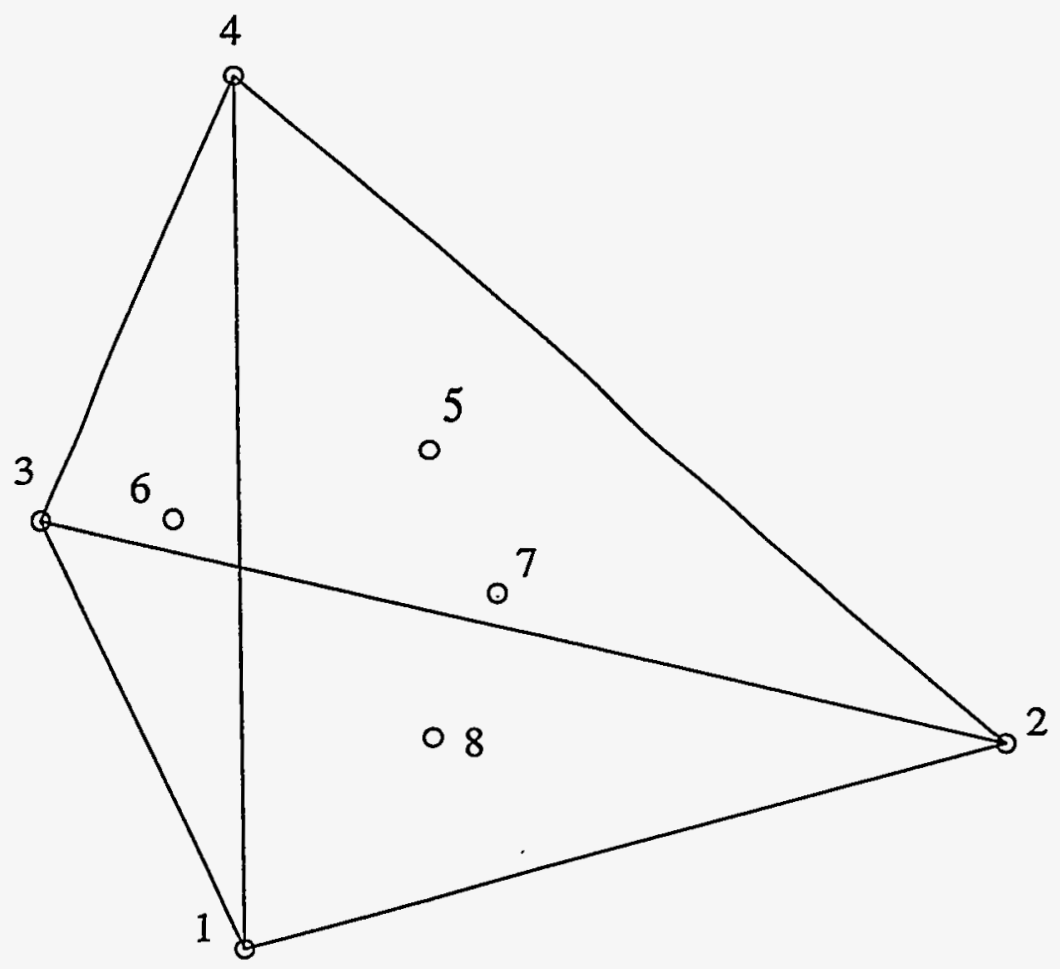

Figure 3: Element geometry of uniform strain tetrahedron. 


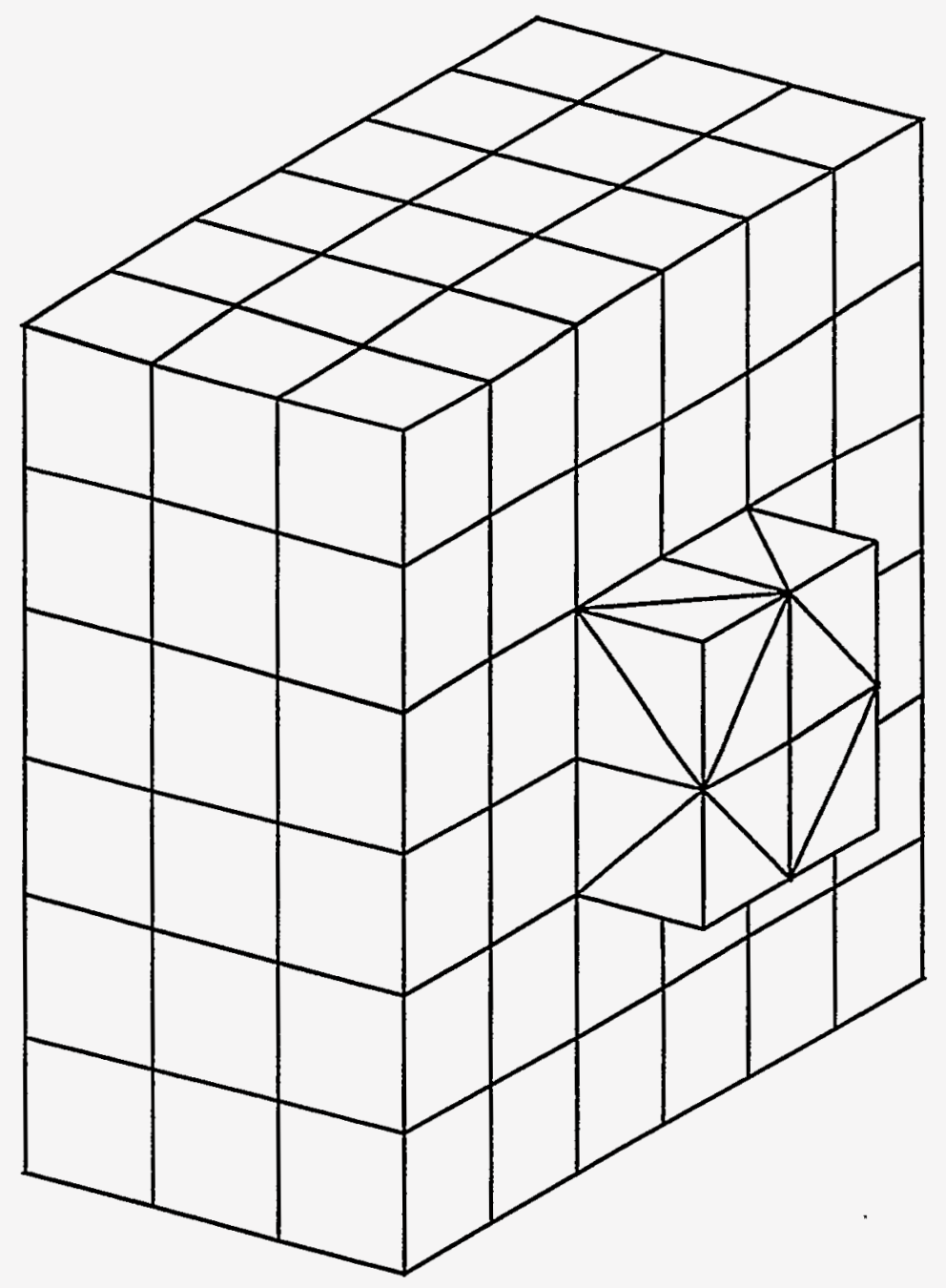

Figure 4: Mesh $6 m$ with 12 transition elements and 92 hexahedral elements removed. 


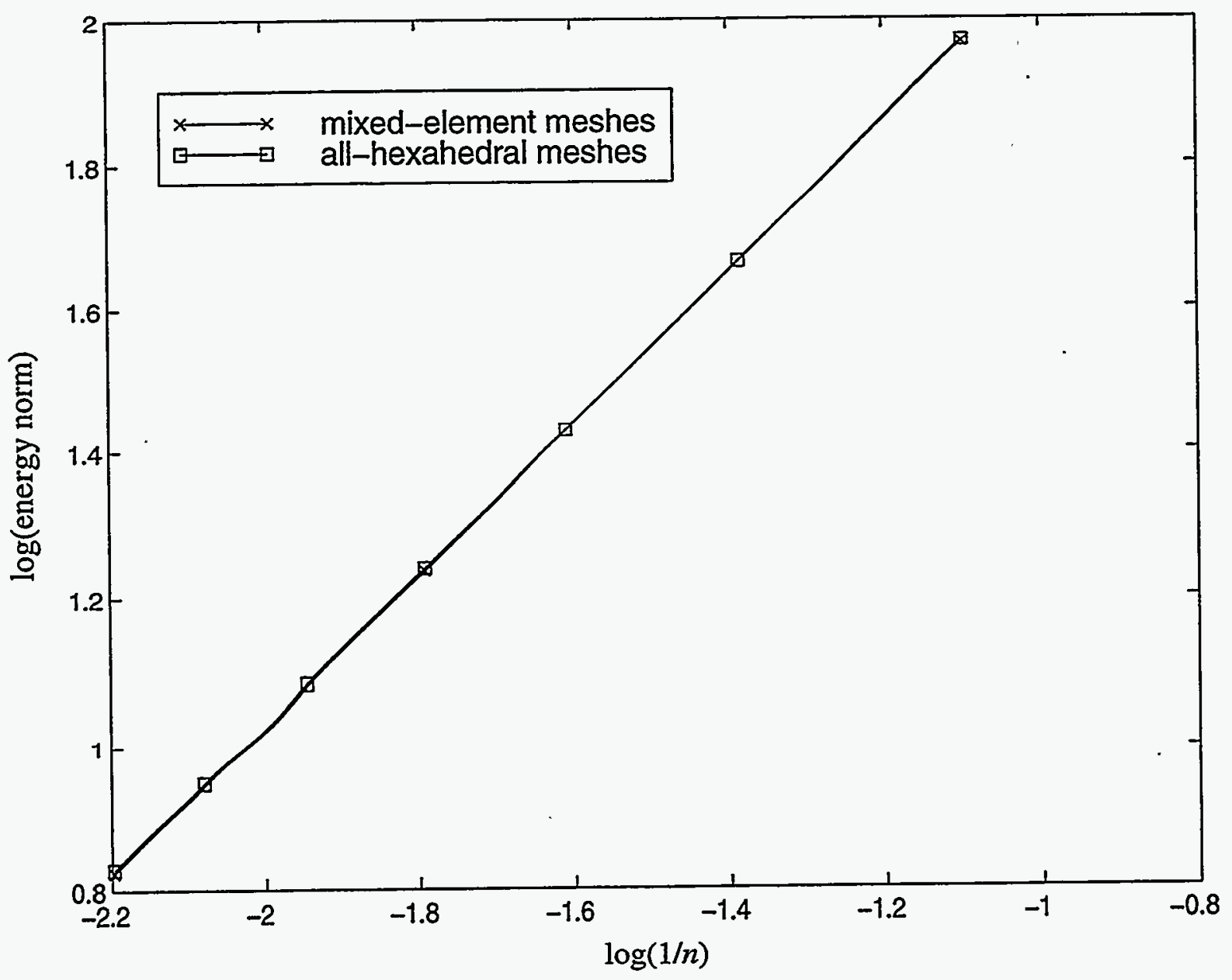

Figure 5: Energy norm of the error for Example $1(v=0.3)$. 


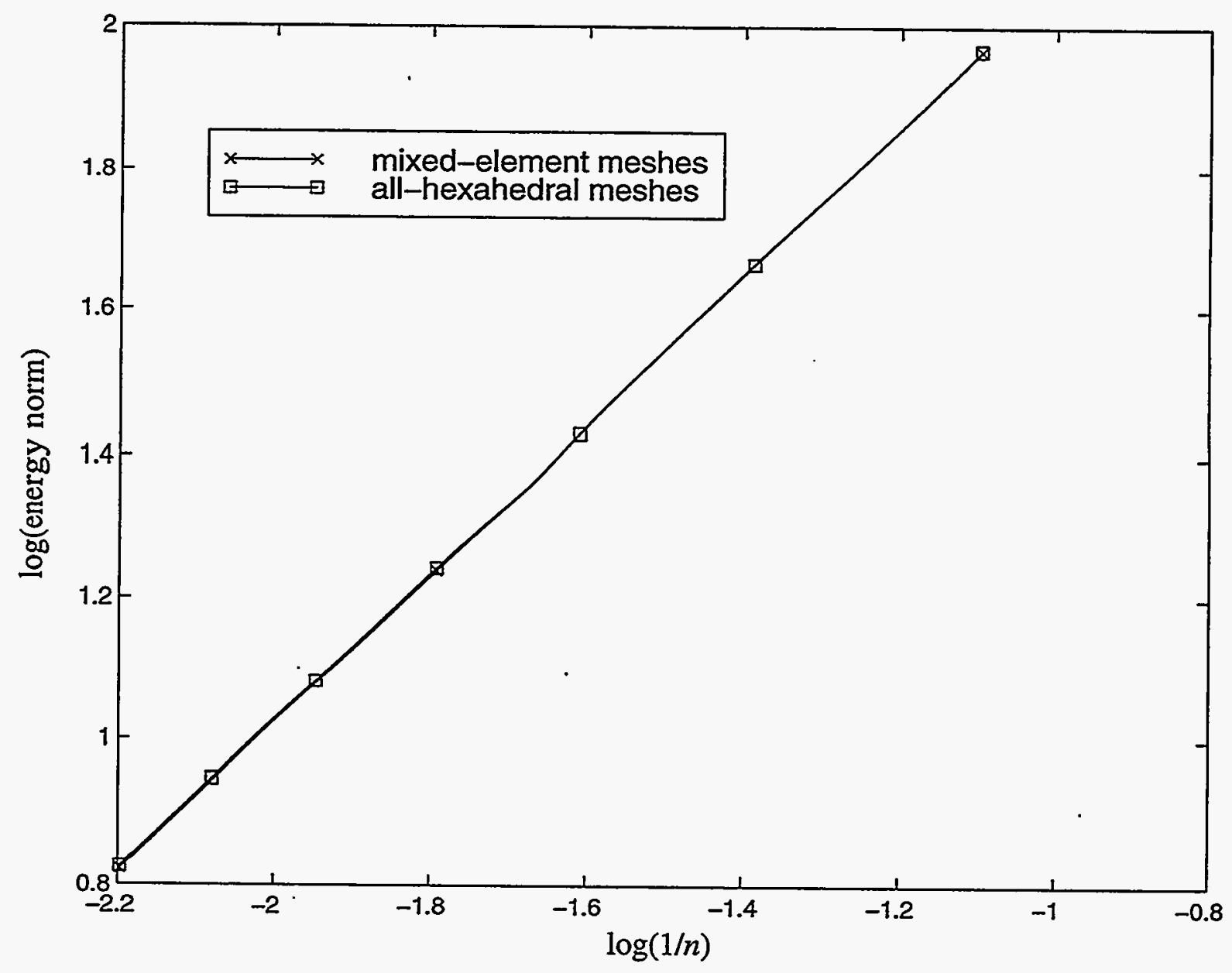

Figure 6: Energy norm of the error for Example $1(v=0.4999)$. 


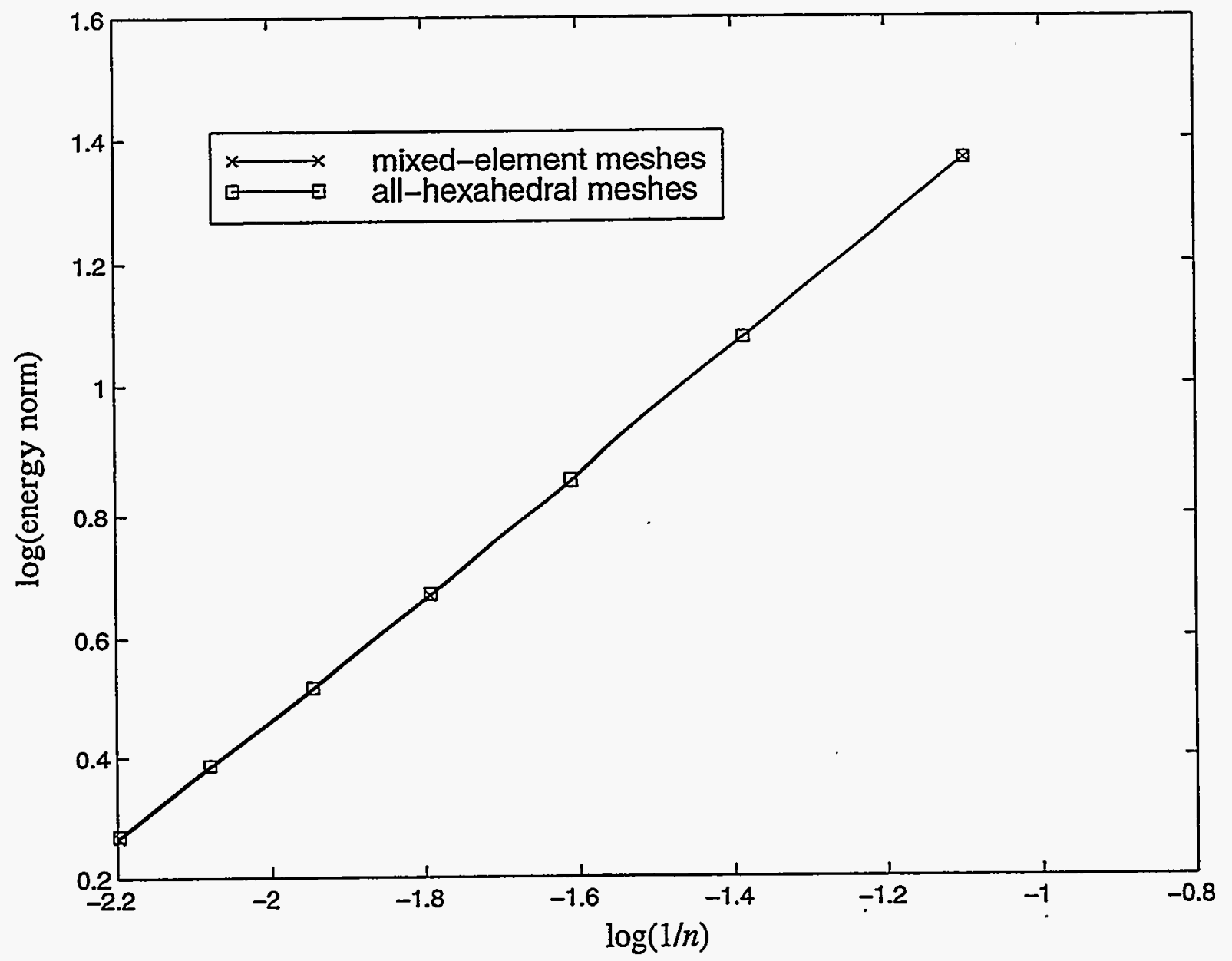

Figure 7: Energy norm of the error for Example $2(v=0.3)$. 


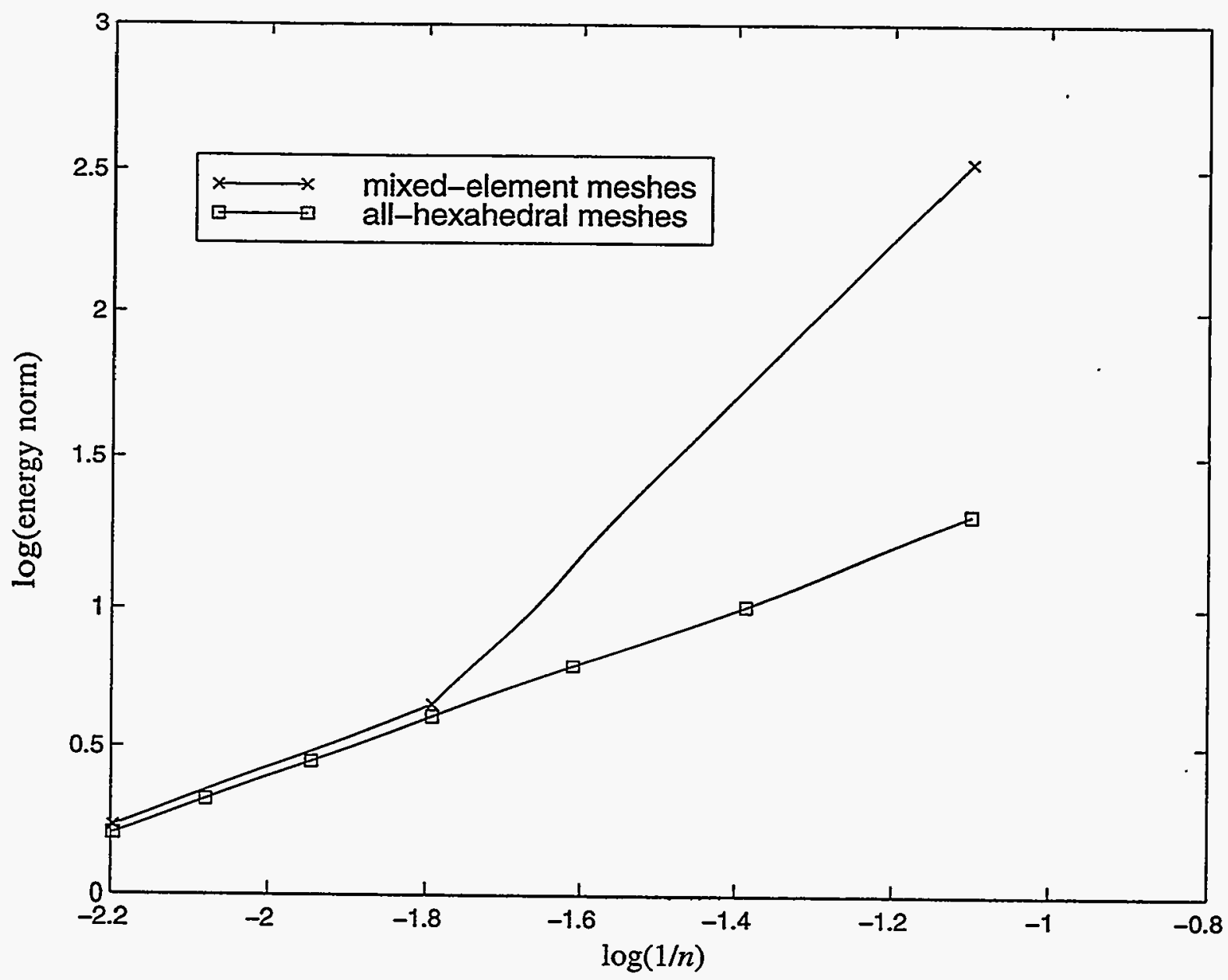

Figure 8: Energy norm of the error for Example $2(v=0.4999)$. 


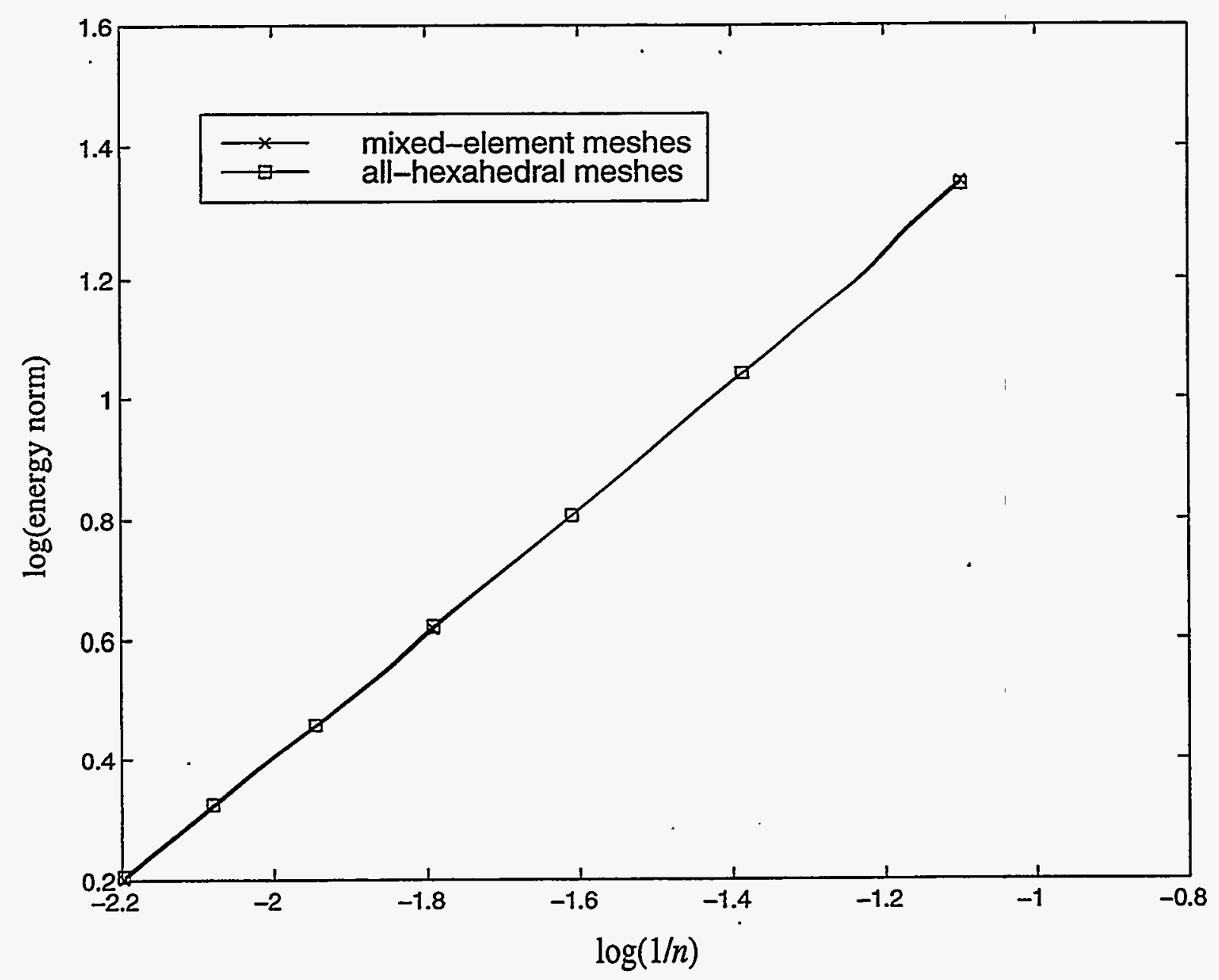

Figure 9: Energy norm of the error for Example 2 using stress rather than displacement boundary conditions $(v=0.4999)$. 


\title{
A Method for Connecting Dissimilar Finite Element Meshes in Two Dimensions ${ }^{1}$
}

\author{
C. R. Dohrmann ${ }^{2}$ \\ S. W. Key ${ }^{3}$ \\ M. W. Heinstein ${ }^{3}$
}

\begin{abstract}
A method is presented for connecting dissimilar finite element meshes in two dimensions. The method combines the concept of master and slave boundaries with the uniform strain approach for finite elements. By modifying the definition of the slave boundary, corrections can be made to element formulations such that first-order patch tests are passed. The method can be used to connect meshes which use different element types. In addition, master and slave boundaries can be designated independently of relative mesh resolutions. Example problems in two-dimensional linear elasticity are presented.
\end{abstract}

Key Words. Finite elements, uniform strain, contact.

\footnotetext{
${ }^{1}$ Sandia is a multiprogram laboratory operated by Sandia Corporation, a Lockheed Martin Company, for the United States Department of Energy under Contract DE-AL04-94AL8500.

${ }^{2}$ Structural Dynamics Department, Sandia National Laboratories, MS 0439, Albuquerque, New Mexico 87185-0439, ernail: crdohrm@sandia.gov, phone: (505) 844-8058, fax: (505) 844-9297.

${ }^{3}$ Engineering and Manufacturing Mechanics Department, Sandia National Laboratories, MS 0443, Albuquerque, New Mexico 87185-0443.
} 


\section{Introduction}

In order to perform a finite element analysis, one may be required to connect two meshes at a shared boundary. Such requirements are common in the assembly of system models from separate subsystem models. One approach to connecting the meshes is to ensure that both meshes have the same number of nodes, the same nodal coordinates, and the same interpolation functions at the shared boundary. If these requirements are met, then the two meshes can be connected simply by equating the degrees of freedom of corresponding nodes at the shared boundary. As might be expected, ensuring conformity between meshes in this manner often requires a significant amount of time and effort in mesh generation.

An alternative to such an approach is to use the concept of master and slave boundaries to connect the meshes. With this concept, one of the connecting mesh boundaries is designated as the master boundary and the other as the slave boundary. For problems in solid mechanics, the meshes are connected by constraining the nodes on the slave boundary to lie on the master boundary. Although this approach is appealing because of its simplicity, overlaps and gaps may develop between the two meshes. For example, a node on the master boundary may either penetrate or pull away from the slave boundary even though the slave node constraints are all satisfied (see Figure 6). As a result, displacement continuity may not hold at all locations on the master-slave interface.

Several different methods have been proposed to connect finite elements or meshes of elements. Mesh grading approaches allow two or more finer elements to abut the edge of a neighboring coarser element [1]. Although these approaches generate conforming boundaries, they are not applicable to the general problem of connecting two dissimilar meshes. Other approaches for connecting meshes [2] also exist, but they generally result in nonconforming boundaries. Finite element approaches developed specifically for contact problems can also be used to connect meshes. These methods [3] include: (i) Lagrange multiplier methods; (ii) penalty methods; and (iii) mixed (or hybrid) methods. Many of these methods are based in part on the master-slave concept.

Regardless of which method is used, it is important to consider the issues associated with continuity at the mesh boundaries. One such issue is the first-order patch test [4]. In general, meshes that are connected using constraint equations or penalty functions alone fail the patch test. The present method differs fundamentally from others by modifying the definition of the slave boundary to ensure satisfaction of the patch test. The basic idea is to replace the geometric definition of the slave boundary with that of the master boundary. The same idea was used recently at the element level to develop a transition element [5].

Enforcement of continuity across mesh boundaries presents several challenges. For example, consider a problem in which the master and slave boundaries initially conform to each another prior to any deformations. Displacements are interpolated quadratically over each element edge on the master boundary. On the slave boundary, displacements are interpolated linearly over each element edge. One is immediately faced with the fact that the two meshes will remain conforming only if the nodal displacements are consistent with a linear displacement field. Similar problems may arise even if the elements on the mesh boundaries use the same orders of interpolation. This fact is demonstrated with a simple 
example problem in Section 3.

The present method combines the master-slave concept with the uniform strain approach for finite elements [6]. For element edges on the slave boundary, nodes at the ends of the edges are constrained to the master boundary. Intermediate nodes along these edges may also be constrained to the master boundary, but such constraints are not required. By properly modifying the formulations of elements on the slave boundary, one can ensure that first-order patch tests are passed. Consequently, results obtained using the method will converge with mesh refinement.

A useful feature of the method is the freedom to designate the master and slave boundaries independently of the resolutions of the two meshes. Standard practice commonly requires the master boundary to have fewer numbers of nodes than the slave boundary. The present method allows one to specify either of the mesh boundaries as master while still satisfying the patch test. It is shown in Section 3 that improved accuracy can be achieved in certain instances by allowing the master boundary to have a greater number of nodes. Thus, there may a preferred choice for the master boundary in certain cases. Methods of mesh refinement based on subdivision of existing elements may also benefit from the method. For example, kinematic constraints on improper nodes could be removed while preserving displacement continuity between adjacent elements.

Details of the method are presented in the following section. The presentation includes a discussion of the uniform strain approach and the geometric concepts upon which the method is based. Example problems in two-dimensional linear elasticity are presented in Section 3. These examples highlight the various capabilities and performance of the method. Comparisons are made with the standard master-slave approach to demonstrate the superior convergence rates of the method.

\section{Formulation}

Consider a generic finite element in two dimensions with nodal coordinates $x_{i I}$ and nodal displacements $u_{i I}$ for $i=1,2$ and $I=1, \ldots, N$. The spatial coordinates and displacements of a point in the global coordinate direction $X_{i}$ are denoted by $x_{i}$ and $u_{i}$, respectively. For isoparametric elements, the same interpolation functions are used for the coordinates and displacements. That is,

$$
\begin{aligned}
& x_{i}=x_{i I} \phi_{I}\left(\eta_{1}, \eta_{2}\right) \\
& u_{i}=u_{i I} \phi_{I}\left(\eta_{1}, \eta_{2}\right)
\end{aligned}
$$

where $\phi_{I}$ is the shape function of node $I$ and $\left(\eta_{1}, \eta_{2}\right)$ are isoparametric coordinates. A summation over all possible values of repeated indices in Eqs. (1-2) and elsewhere is implied unless noted otherwise.

The Jacobian determinant $J$ of the element is defined as

$$
J=\operatorname{det}\left[\begin{array}{ll}
\partial x_{1} / \partial \eta_{1} & \partial x_{2} / \partial \eta_{1} \\
\partial x_{1} / \partial \eta_{2} & \partial x_{2} / \partial \eta_{2}
\end{array}\right]
$$


The area $A$ of the element can be expressed in terms of $J$ by

$$
A=\int_{A_{\eta}} J d A
$$

where $A_{\eta}$ is the area of integration of the element in the isoparametric coordinate system.

It is assumed that $A$ is a homogeneous function of the nodal coordinates. It is also assumed that a linear displacement field can be expressed exactly in terms of the shape functions. Under these conditions, the uniform strain approach of Ref. 6 states that the nodal forces $f_{I I}$ associated with element stresses are given by

$$
f_{i I}=h \sigma_{i j} B_{j I}
$$

where $h$ is the element thickness, $\sigma_{i j}$ are components of the Cauchy stress tensor (assumed constant throughout the element), and

$$
B_{j I}=\frac{\partial A}{\partial x_{j I}}
$$

In addition. one has

$$
A=x_{j I} B_{j I} \quad \text { for } \quad j=1,2
$$

where there is no summation over the index $j$ in Eq. (7). Closed-form expressions for $B_{j I}$ are presented in Ref. 6 for the four-node quadrilateral. For purposes of completeness, similar expressions are included in the Appendix for a variety of triangular and quadrilateral elements.

Following the development in Ref. 6, one can show that

$$
\int_{\Omega} \frac{\partial \phi_{I}}{\partial x_{j}} d A=B_{j I}
$$

where $\Omega$ is the domain of the element in the global coordinate system. Based on Eq. (8), the uniform strain $\epsilon^{u}$ of the element is expressed in terms of nodal displacements as

$$
\epsilon^{u}=C u
$$

where

$$
\begin{gathered}
\epsilon^{u}=\left[\begin{array}{c}
\epsilon_{11}^{u} \\
\epsilon_{22}^{u} \\
\gamma_{12}^{u}
\end{array}\right] \\
C=\frac{1}{A}\left[\begin{array}{ccccccc}
B_{11} & 0 & B_{12} & 0 & \cdots & B_{1 N} & 0 \\
0 & B_{21} & 0 & B_{22} & \cdots & 0 & B_{2 N} \\
B_{21} & B_{11} & B_{22} & B_{21} & \cdots & B_{2 N} & B_{1 N}
\end{array}\right]
\end{gathered}
$$

and

$$
u=\left[\begin{array}{lllllll}
u_{11} & u_{21} & u_{12} & u_{22} & \cdots & u_{1 N} & u_{2 N}
\end{array}\right]^{T}
$$


Elements based on the uniform strain approach have the appealing feature that they pass first-order patch tests.

Boundaries of two-dimensional elements are defined either by straight or curved lines. Elements with interpolation functions that vary linearly along the edges, e.g. the three-node triangle and four-node quadrilateral, have straight boundaries. In contrast, elements with quadratic or higher-order interpolation functions, e.g. the six-node triangle and eight-node serendipity quadrilateral, generally have curved boundaries. That being the case, it may not be obvious how to connect two meshes which use different orders of interpolation along their boundaries.

Difficulties can arise using the standard master-slave concept even if the boundaries of both meshes are defined by straight lines. As was mentioned previously, there may not be any constraints to keep a node on the master boundary from penetrating or pulling away from the slave boundary. Such problems are addressed with the present method by requiring the edges of elements on the slave boundary to always conform to the master boundary. In order to explain how this is done, some preliminary geometric concepts are introduced first.

Notice from Eqs. (6), (9) and (11) that the relationship between strain and displacement for a uniform strain element is defined completely by its area. Consequently, the uniform strain characteristics of two elements are identical if the expressions for their areas are the same. This fact is important because it allows one to consider alternative interpolation functions for elements with edges on the master and slave boundaries. By doing so, one can interpret the present method as an approach for generating conforming finite elements at the shared boundary.

Consider the eight-node serendipity quadrilateral shown in Figure 1a. Each point on an edge of the element is associated with a specific value of an isoparametric coordinate. Both the spatial coordinates and displacements of the point are linear functions of the coordinates and displacements of the three nodes defining the edge. The specific forms of these relationships are obtained by setting either $\eta_{1}$ or $\eta_{2}$ equal to one of its bounding values in Eqs. (1-2).

The dashed lines in Figures $1 \mathrm{~b}$ show an alternative geometric description of the element. Each vertex of the sixteen-sided polygon intersects the curved edges of the original eightnode quadrilateral. Although the precise location of center node $c$ is not important, its coordinates may be expressed in terms of the others as

$$
x_{i c}=\left(x_{i 5}+x_{i 6}+x_{i 7}+x_{i 8}\right) / 2-\left(x_{i 1}+x_{i 2}+x_{i 3}+x_{i 4}\right) / 4
$$

The domain of the polygon in Figure $1 \mathrm{~b}$ is divided into sixteen triangular regions. Within each of these regions the interpolation functions are linear. In other words, the displacement of a point in a triangular region is determined by its location and the displacements of the three nodes defining the triangle. One may approximate the boundary of the original quadrilateral to any level of accuracy by increasing the number vertices of the polygon. As the number of vertices approaches infinity, the element boundaries in Figures $1 \mathrm{a}$ and $1 \mathrm{~b}$ become identical.

Although the two elements in Figure 1 are significantly different, their uniform strain 
characteristics are approximately the same. In the limit as the number of boundary vertices in Figure $1 \mathrm{~b}$ approaches infinity, the uniform strain characteristics are identical. By viewing all the element edges on the master and slave boundaries as connected straight-line segments, one can develop a systematic method for connecting the two meshes that passes first-order patch tests. We note that the alternative element description shown in Figure $1 b$ satisfies the basic assumptions of the uniform strain approach. That is, the element area is a homogeneous function of the nodal coordinates and a linear displacement field can be expressed exactly in terms of the interpolation functions.

We are now in a position to present the method for modifying the formulations of elements with edges on the slave boundary. Changes to elements with edges on the master boundary are not required. The concept of alternative piecewise-linear interpolation functions was introduced in the previous paragraphs to facilitate interpretation of the present method as an approach for generating conforming elements at the master-slave interface. These alternative interpolation functions are never used explicitly to modify the element formulations.

Figure 2 shows a typical element with an edge $E_{1}$ on the slave boundary. Nodes 1 and $Q$ on $E_{1}$ are constrained to the master boundary. Any intermediate nodes along $E_{1}$ may or may not be constrained to the master boundary; the choice is up to the analyst. Nodes on the master boundary are also shown in Figure 2. The segment of the master boundary bounded by points $1^{*}$ and $Q^{*}$ is designated as $E_{m}$. Node 1 is constrained to point $1^{*}$ and node $Q$ is constrained to point $Q^{*}$.

Based on their initial proximity to the master boundary, the coordinates of nodes on the slave boundary can be expressed as

$$
x_{i M}=a_{M K} x_{i K}
$$

for $M=1, \ldots, Q$. If node $M$ is constrained to the master boundary, then the index $K$ in Eq. (14) ranges over all the nodes defining $E_{m}$. If node $M$ is not constrained to the master boundary, then $a_{M K}=\delta_{M K}$ where $\delta$ is the Kronecker delta.

The basic idea of the following development is to replace $E_{1}$ with $E_{m}$. By doing so, one can ensure that no overlaps or gaps develop between the two meshes. Using Green's theorem, element area can be expressed in terms of line integrals along the edges as

$$
A=\sum_{k=1}^{N_{e}} \int_{E_{k}} x_{1} d x_{2}
$$

where $N_{e}$ is the number of element edges and $E_{k}$ denotes edge $k$. Let $\hat{A}$ denote the area of a uniform strain element obtained by replacing $E_{1}$ with $E_{m}$. It follows from Eq. (15) that

$$
\hat{A}=A-\int_{E_{1}} x_{1} d x_{2}+\int_{E_{m}} x_{1} d x_{2}
$$

The analog to Eq. (6) for the uniform strain element is given by

$$
\hat{B}_{j \hat{I}}=\frac{\partial \hat{A}}{\partial x_{j \hat{I}}}
$$


The index $\hat{I}$ is used instead of $I$ in Eq. (17) to remind the reader that $\hat{A}$ depends on the coordinates of the original element nodes as well as the nodes defining $E_{m}$. To be specific, the index $\hat{I}$ takes on all values of $I$ for the original element except the numbers of nodes constrained to the master boundary. In addition, $\hat{I}$ takes on the numbers of all nodes defining $E_{m}$.

Substituting Eqs. (14) and (16) into Eq. (17) and using the chain rule for differentiation, one obtains

$$
\hat{B}_{j \hat{I}}=B_{j \hat{I}}+B_{j \bar{M}} a_{\tilde{M} \hat{I}}-\frac{\partial}{\partial x_{j \hat{I}}} \int_{E_{1}} x_{1} d x_{2}+\frac{\partial}{\partial x_{j \hat{I}}} \int_{E_{m}} x_{1} d x_{2}
$$

where the index $\tilde{M}$ takes on the numbers of nodes constrained to the master boundary. Notice that $B_{j j}=0$ if $\hat{I}$ refers to a node on the master boundary. In addition, $a_{\tilde{M} \hat{I}}$ is zero if $\hat{I}$ refers to node numbers of the original element.

The line integrals on the right hand side Eq. (18) can be evaluated using either closedform expressions or by numerical integration. For example, if an edge on the slave boundary has two nodes. i.e. $Q=2$, then

$$
\frac{\partial}{\partial x_{\jmath j}} \int_{E_{1}} x_{1} d x_{2}=\left\{\begin{array}{cc}
\left(x_{22}-x_{21}\right) / 2 & j=1 \text { and } \hat{I}=1 \\
\left(x_{22}-x_{21}\right) / 2 & j=1 \text { and } \hat{I}=2 \\
-\left(x_{11}+x_{12}\right) / 2 & j=2 \text { and } \hat{I}=1 \\
\left(x_{11}+x_{12}\right) / 2 & j=2 \text { and } \hat{I}=2 \\
0 & \text { otherwise }
\end{array}\right.
$$

and for $Q=3$.

$$
\frac{\partial}{\partial x_{\jmath} i} \int_{E_{1}} x_{1} d x_{2}=\left\{\begin{array}{cc}
-x_{21} / 2+2 x_{22} / 3-x_{23} / 6 & j=1 \text { and } \hat{I}=1 \\
2\left(x_{23}-x_{21}\right) / 3 & j=1 \text { and } \hat{I}=2 \\
x_{21} / 6-2 x_{22} / 3+x_{23} / 2 & j=1 \text { and } \hat{I}=3 \\
-x_{11} / 2-2 x_{12} / 3+x_{13} / 6 & j=2 \text { and } \hat{I}=1 \\
2\left(x_{11}-x_{13}\right) / 3 & j=2 \text { and } \hat{I}=2 \\
-x_{11} / 6+2 x_{12} / 3+x_{13} / 2 & j=2 \text { and } \hat{I}=3 \\
0 & \text { otherwise }
\end{array}\right.
$$

Similar expressions can be derived for element edges with four or more nodes.

In general, $E_{m}$ is composed of one or more element edge segments on the master boundary. The coordinates of points along any one of these segments can be expressed in terms of the shape functions $\psi_{K}$ for the edge as

$$
x_{i}=x_{i K} \psi_{K}(\eta)
$$

where the index $K$ ranges over the numbers of nodes defining the element edge. It follows from Eq. (21) that

$$
\frac{\partial}{\partial x_{j \hat{I}}} \int_{L_{\eta}} x_{1} d x_{2}= \begin{cases}\int_{\eta_{l}}^{\eta_{u}} x_{2 K} \psi_{\hat{I}}\left(\partial \psi_{K} / \partial \eta\right) d \eta & j=1 \\ \int_{\eta_{l}}^{\eta_{u}} x_{1 K} \psi_{K}\left(\partial \psi_{\hat{I}} / \partial \eta\right) d \eta & j=2\end{cases}
$$


where $L_{\eta}$ denotes the integration domain of the edge segment and $\eta_{l}$ and $\eta_{u}$ define its ends. The second integral in Eq. (18) can be evaluated by summing the contributions of all such edge segments defining $E_{m}$.

If the slave boundary consists entirely of uniform strain elements, then all the necessary corrections are contained in $\hat{B}_{j \hat{I}}$. By using Eq. (18) to calculate $\hat{B}_{j \hat{I}}$ for elements on the slave boundary, one can perform analyses of connected meshes for both linear and nonlinear problems. A general method of hourglass control [7] can also be used to stabilize any elements on the boundary with spurious zero energy deformation modes.

The remainder of this section is concerned with extending the method to accommodate more commonly used finite elements on the slave boundary. Although we believe the method can be extended easily to nonlinear problems, attention is restricted presently to the linear case. Needless to say, many problems of practical interest are in this category.

Prior to any modifications, the stiffness matrix $K$ of an element on the slave boundary can be expressed as

$$
K=K_{u}+K_{r}
$$

where $K_{u}$ denotes the uniform strain portion of $K$ and $K_{r}$ is the remainder. The matrix $K_{u}$ is defined as

$$
K_{u}=A C^{T} D C
$$

where $D$ is a material matrix that is assumed constant throughout the element. Recall that $A$ is the element area and $C$ is given by Eq. (11). Substituting Eq. (24) into Eq. (23) and solving for $K_{r}$ yields

$$
K_{r}=K-A C^{T} D C
$$

Let $u^{l}$ denote the vector $u$ (see Eq. 12) obtained by sampling a linear displacement field at the nodes. The nodal forces $f^{l}$ associated with $u^{l}$ are given by

$$
f^{l}=K u^{l}
$$

For a properly formulated element, one has

$$
K_{u} u^{l}=f^{l}
$$

and

$$
K_{r} u^{l}=0
$$

If Eq. (27) does not hold, then $K_{u} u^{l} \neq f^{l}$ and elements based on the uniform strain approach would fail a first-order patch test. Equation (28) implies that $K_{r}$ does not contribute to the nodal forces for linear displacement fields.

The basic idea of the following development is to alter the uniform strain portion of the stiffness matrix while leaving $K_{r}$ unchanged. Let $\hat{u}$ denote the displacement vector for nodes associated with the index $\hat{I}$ (see discussion following Eq. 17). Based on the constraints in Eq. (14), one may express $u$ in terms of $\hat{u}$ as

$$
u=G \hat{u}
$$


where $G$ is a transformation matrix. The modified stiffness matrix $\hat{K}$ of the element is defined as

$$
\hat{K}=\hat{A} \hat{C}^{T} D \hat{C}+G^{T} K_{r} G
$$

where $\hat{C}$ denotes the matrix $C$ (see Eq. 11) associated with $\hat{B}_{j \hat{I}}$ (see Eq. 18). The stiffness matrix $K_{m s}$ obtained using the standard master-slave approach is given by

$$
K_{m s}=G^{T} \dot{K} G
$$

Comparing $\hat{K}$ with $K_{m s}$, one finds that

$$
\hat{K}-K_{m s}=\hat{A} \hat{C}^{T} D \hat{C}-G^{T}\left(A C^{T} D C\right) G
$$

The right hand side of Eq. (32) is simply the difference between the uniform strain portions of $\hat{K}$ and $K_{m s}$. If continuity at the master-slave interface holds by satisfying Eq. (29) alone, then the two integrals in Eq. (18) cancel each other and $\hat{K}=K_{m s}$. Thus, under such conditions, the present method and the standard master-slave approach are equivalent.

Prior to element modifications, the strain $\epsilon$ in an element on the slave boundary can be expressed as

$$
\epsilon=\mathrm{Cu}+\mathrm{Hu}
$$

where $C u$ is the uniform strain (see Eq. 9) and $H u$ is the remainder. The modified element strain $\hat{\epsilon}$ is defined as

$$
\hat{\epsilon}=\hat{C} \hat{u}+H u
$$

Equation (34) is used to calculate the strains in elements with edges on the slave boundary.

One might also consider developing a modified stiffness matrix $\hat{K}_{\hat{\epsilon}}$ based on Eq. (34). The result is

$$
\hat{K}_{\hat{\epsilon}}=\hat{A} \hat{C}^{T} D \hat{C}+\int_{\hat{\Omega}}\left[\hat{C}^{T} D H G+G^{T} H^{T} D \hat{C}+G^{T} H^{T} D H G\right] d A
$$

where $\hat{\Omega}$ denotes the domain of the element with edge $E_{1}$ replaced by $E_{m}$. The difficulties with using $\hat{K}_{\hat{\epsilon}}$ for an element formulation are twofold. First, it may not be simple to evaluate the integral in Eq. (35) because the domain $\hat{\Omega}$ could be irregular. Second, and more importantly, such an element formulation does not pass the patch test. To explain this fact, let $\hat{u}^{l}$ denote the vector $\hat{u}$ obtained by sampling a linear displacement field. In general, one has $\hat{K} \hat{u}^{l} \neq \hat{K}_{\hat{\epsilon}} \hat{u}^{l}$ since the product $\hat{C} \hat{u}^{l}$ is not necessarily zero.

The basic idea of modifying the definition of the slave boundary can also be used to connect dissimilar finite element meshes in three dimensions. The extensions are fairly straightforward and have been applied successfully to problems in three-dimensional elasticity. These extensions along with results for a variety of problems are the topic of a forthcoming paper.

\section{Example Problems}

All the example problems in this section assume small deformations of a linear, elastic, isotropic material with Young's modulus $E=10^{7}$ and Poisson's ratio $\nu=0.3$. In this case, 
the material matrix $D$ for plane stress can be expressed as

$$
D=\frac{E}{1-\nu^{2}}\left[\begin{array}{ccc}
1 & \nu & 0 \\
\nu & 1 & 0 \\
0 & 0 & (1-\nu) / 2
\end{array}\right]
$$

Six different element types shown in Figure 3 are considered in the example problems. These include the four-node quadrilateral $(Q 4)$, eight-node quadrilateral $(Q 8)$, twelve-node quadrilateral $(Q 12)$, three-node triangle (T3), six-node triangle (T6), and ten-node triangle (T10). Stiffness matrices of the various elements are calculated using numerical integration. The quadrilateral elements use 2 by 2,3 by 3 , and 4 by 4 Gaussian quadrature for $Q 4, Q 8$ and $Q 12$, respectively. Numerical integration formulae for triangles (see Ref. 4) with 1, 3 and 7 points are used for $T 3, T 6$ and $T 10$, respectively.

Two meshes connected at a shared boundary are used in all the example problems. Mesh 1 is initially bounded by the the four sides $x_{1}=0, x_{1}=h_{1}, x_{2}=0$ and $x_{2}=h_{2}$ while Mesh 2 is initially bounded by the four sides $x_{1}=h_{1}, x_{1}=2 h_{1}, x_{2}=0$ and $x_{2}=h_{2}$. The two meshes consist of either quadrilateral or triangular elements as shown in Figure 4 . The number of element edges in direction $i$ for mesh $m$ is designated as $n_{i m}$. Thus, all the meshes in Figure 4 have $n_{11}=n_{21}=2$ and $n_{12}=n_{22}=3$. Mesh configurations are designated by the element type for Mesh 1 followed by the element type for Mesh 2 (see Figure 4).

Calculated values of the energy norm of the error are presented in the example problems for purposes of comparison and for the investigation of convergence rates. The energy norm of the error is a measure of the accuracy of a finite element approximation and is defined as

$$
e=\left[\sum_{k \in \mathcal{I}} \int_{\Omega_{k}}\left(\epsilon^{f e}-\epsilon^{e x a c t}\right)^{T} D\left(\epsilon^{f e}-\epsilon^{e x a c t}\right) d A\right]^{1 / 2} .
$$

where $\Omega_{k}$ is the domain of element $k$ and $\epsilon^{f e}$ and $\epsilon^{\text {exact }}$ denote the finite element and exact strains, respectively. The symbol $\mathcal{I}$ denotes the set of all element numbers for the two meshes. Calculation of energy norms for the quadrilateral and triangular elements is based on the integration schemes for element types $Q 12$ and $T 10$, respectively.

Results are also presented for an energy norm density $e_{b}$ of the error defined as

$$
e_{b}=\left[\frac{1}{A_{b}} \sum_{k \in \mathcal{I}_{b}} \int_{\Omega_{k}}\left(\epsilon^{f e}-\epsilon^{\text {exact }}\right)^{T} D\left(\epsilon^{f e}-\epsilon^{\text {exact }}\right) d A\right]^{1 / 2}
$$

where $A_{b}$ denotes the sum of the areas of all elements with edges on the master-slave interface. The symbol $\mathcal{I}_{b}$ denotes the set of all element numbers associated with $A_{b}$.

\section{Example 3.1}

The first example focuses on a uniaxial tension patch test and highlights some of the differences between the standard master-slave and present approaches. The boundary conditions for the problem are given by

$$
u_{1}\left(0, x_{2}\right)=0
$$




$$
u_{2}(0,0)=0
$$

and

$$
\sigma_{11}\left(2 h_{1}, x_{2}\right)=1
$$

The exact solution is given by

$$
\begin{aligned}
& u_{1}\left(x_{1}, x_{2}\right)=x_{1} / E \\
& u_{2}\left(x_{1}, x_{2}\right)=-\nu x_{2} / E
\end{aligned}
$$

All the meshes used in the example have $h_{1}=5, h_{2}=10, n_{11}=n_{21}=n$ and $n_{12}=\dot{n}_{22}=$ $3 n / 2$ where $n$ is a positive even integer.

Several analyses with $n=2$ were performed to evaluate the method. Using all six element types for Mesh 1 and Mesh 2 resulted in 36 different mesh configurations. Nodes internal to the meshes and along the master-slave interface were moved randomly so that all the elements were initially distorted. Following the initial movement of nodes, nodes on the slave boundary were repositioned to lie on the master boundary. Intermediate nodes on the slave boundary for quadratic and cubic elements were either constrained to the master boundary or left unconstrained. In addition, the two meshes were alternately designated as master and slave. In all cases the patch test was passed. That is, the calculated element stresses and nodal displacements were in agreement with the exact solution.

The meshes shown in Figure 4 do not satisfy first-order patch tests if the standard masterslave approach is used. To help explain why this is the case, consider the meshes shown in Figure 5 . The boundary conditions given by Eqs. (39-41) should result in a state of uniaxial stress in the $X_{1}$ direction equal to unity. According to the state of stress in element number 4 , the force at node 9 in the $X_{1}$ direction equals $h_{2} / 4$. Based on the stresses in elements 11 and 8 , the nodal forces in the negative $X_{1}$ direction at nodes 22 and 18 equal $h_{2} / 6$ and $h_{2} / 3$, respectively. Constraining node 18 on the slave boundary to the master boundary implies that the displacement of node 18 equals to two thirds the displacement of node 6 plus one third the displacement of node 9 . Thus, the equivalent nodal force at node 9 due to elements 11 and 8 equals $h_{2} / 6+(1 / 3) h_{2} / 3=5 h_{2} / 18$. An imbalance in forces at node 9 clearly exists. An exaggerated plot of the displaced geometry is shown in Figure 6. Notice that a gap develops between the two meshes even though the slave node constraints are all satisfied.

The remaining discussion for this example concerns results obtained using the standard master-slave approach with Mesh 1 designated as master. The stress component $\sigma_{11}$ at centroids of elements on the slave boundary is plotted versus $x_{2}$ in Figure 7 for mesh configuration $Q 4 Q 4$. Notice that $\sigma_{11}$ alternates from below to above its exact value as $x_{2}$ is varied. It is clear from the figure that refinement of both meshes does not improve the accuracy of the solution at the shared boundary. Similar results for mesh configuration $Q 8 Q 8$ are shown in Figure 8. Comparison of Figures 7 and 8 shows that the errors in stress at the slave boundary are greater for mesh configuration $Q 8 Q 8$ than for $Q 4 Q 4$.

Plots of the energy norm of the error for mesh configurations $Q 4 Q 4$ and $Q 8 Q 8$ are shown in Figure 9. It is clear that the energy norms decrease with mesh refinement, but the 
convergence rates are significantly lower than those for elements in a single unconnected mesh. The slopes of lines connecting the first and last data points are approximately 0.51 and 0.50 for $Q 4 Q 4$ and $Q 8 Q 8$, respectively. In contrast, the energy norms of the error for a single mesh of $Q 4$ or undistorted $Q 8$ elements have slopes of 1 and 2, respectively, in the absence of singularities. The fact that displacement continuity is not satisfied at the shared boundary severely degrades the convergence characteristics of the connected meshes.

\section{Example 3.2}

The second example investigates the convergence rates for the method. The specific problem considered is pure bending. The problem description is identical to Example 3.1 with the exception that the boundary condition at $x_{1}=2 h_{1}$ is replaced by

$$
\sigma_{11}\left(2 h_{1}, x_{2}\right)=h_{2} / 2-x_{2}
$$

The exact solution for stresses is given by

$$
\begin{aligned}
& \sigma_{11}\left(x_{1}, x_{2}\right)=h_{2} / 2-x_{2} \\
& \sigma_{22}\left(x_{1}, x_{2}\right)=0 \\
& \sigma_{12}\left(x_{1}, x_{2}\right)=0
\end{aligned}
$$

In all cases Mesh 1 was designated as master. Results presented for mesh configuration Q8Q8 were obtained by constraining all mid-edge nodes on the slave boundary to the master boundary.

Plots of the energy norm of the error are shown in Figure 10 for mesh configurations $Q 4 Q 4$ and $Q 8 Q 8$. The slopes of lines connecting the first and last data points are approximately 1.00 and 1.56 for $Q 4 Q 4$ and $Q 8 Q 8$, respectively. Notice that the convergence rate of unity for $Q 4$ elements is achieved by mesh configuration $Q 4 Q 4$. Although the convergence rate is greater for mesh configuration $Q 8 Q 8$, the optimal rate of 2 is not achieved. One should not expect to obtain a convergence rate of 2 with the present method since corrections are only made to satisfy first-order patch tests. Nevertheless, mesh configuration $Q 8 Q 8$ exhibits a convergence rate greater than unity.

\section{Example 3.3}

The final example demonstrates the freedom to designate master and slave boundaries independently of the resolutions of the two meshes. The specific problem considered is bending of a beam by a uniform load [8] for mesh configuration $Q 4 Q 4$. The boundary conditions are given by

$$
\begin{array}{r}
u_{1}\left(2 h_{1}, h_{2}\right)=0 \\
u_{2}\left(x_{1}, 0\right)=0
\end{array}
$$

and

$$
\begin{aligned}
\sigma_{11}\left(0, x_{2}\right) & =-1 \\
\sigma_{22}\left(x_{1}, h_{2}\right) & =\left(2 x_{1}^{3} / 3-2 h_{1}^{2} x_{1} / 5\right) /(2 I) \\
\sigma_{12}\left(x_{1}, h_{2}\right) & =-\left(h_{1}^{2}-x_{1}^{2}\right) x_{2} /(2 I)
\end{aligned}
$$


where

$$
I=2 h_{1}^{3} / 3
$$

The exact solution for stresses is given by

$$
\begin{aligned}
& \sigma_{11}=-\left(x_{1}^{3} / 3-h_{1}^{2} x_{1}+2 h_{1}^{3} / 3\right) /(2 I) \\
& \sigma_{22}=\left[\left(h_{2}^{2}-x_{2}^{2}\right) x_{1}+\left(2 x_{1}^{3} / 3-2 h_{1}^{2} x_{1} / 5\right)\right] /(2 I) \\
& \sigma_{12}=-\left(h_{1}^{2}-x_{1}^{2}\right) x_{2} /(2 I)
\end{aligned}
$$

All the meshes used in the example have $h_{1}=1, h_{2}=10, n_{11}=n_{12}=n, n_{21}=5 n$ and $n_{22}=10 n$. Thus, Meshes 1 and 2 have the same resolution in the $X_{1}$ direction while the resolution of Mesh 2 is twice that of Mesh 1 in the $X_{2}$ direction. Two different cases are considered. For Case 1, Mesh 1 is designated as master. For Case 2, Mesh 2 is designated as master. Results for Case 1 are identical to those obtained using the standard master-slave approach since the meshes are conforming in this case.

Plots of the energy norm of the error are shown in Figure 11 for the two cases. Notice that Case 2 is consistently more accurate for all the mesh resolutions considered. In order to investigate the cause of the differences, the energy norm density of the error (see Eq. 38) was calculated at the mesh interface. Results of these calculations are shown in Figure 12. Notice that the energy norm densities for the two cases both have slopes near unity, but Case 2 is more accurate than Case 1. It is thought that Case 2 is more accurate than Case 1 because fewer degrees of freedom are constrained at the shared boundary. This example shows that there is a preferred choice for the master boundary in certain instances.

Differences between the two cases are also illustrated in Figures 13 and 14. These figures show the variation of normalized shear stress at centroids of elements on the slave boundary. The normalized shear stress $\bar{\sigma}_{12}$ is defined as

$$
\bar{\sigma}_{12}=\sigma_{12}^{f e} / \sigma_{12}\left(h_{1} /(2 n), h_{2}\right)
$$

where $\sigma_{12}^{f e}$ denotes the shear stress from the finite element solution and $\sigma_{12}$ is given by Eq. (56). Notice in Figure 13 the abrupt changes in $\bar{\sigma}_{12}$ between adjacent elements for Case 1. It is thought that these changes are caused by constraining the higher resolution slave boundary to the coarser master boundary. These changes become more pronounced as the integer ratio $n_{22} / n_{21}$ or the ratio $n_{21} / n_{11}$ is increased. In contrast, the shear stresses for Case 2 vary smoothly and are in much better agreement with the exact solution. The differences between Cases 1 and 2 are reduced significantly for mesh configuration Q8Q8.

\section{Conclusions}

A straightforward method is presented for connecting dissimilar finite element meshes in two dimensions. By modifying the definition of the slave boundary, corrections can be made to element formulations such that first-order patch tests are passed. The method is used successfully to connect meshes with different element types. In addition, master and slave boundaries can be designated independently of the resolutions of the two meshes. 
A simple uniaxial stress example demonstrated several of the advantages of the present method over the standard master-slave approach. Although the energy norm of the error decreased with mesh refinement for the master-slave approach, the convergence rates were significantly lower than those for elements in a single unconnected mesh. Calculated stresses at the shared boundary had errors up to 6.5 and 12.1 percent for connected meshes of fournode and eight-node quadrilaterals, respectively. Moreover, these errors could not be reduced significantly with mesh refinement.

A convergence rate of unity for the energy norm of the error was achieved for a pure bending example using connected meshes of four-node quadrilateral elements. This convergence rate is consistent with that for a single mesh of four-node quadrilaterals. A convergence rate of approximately 1.56 was achieved for connected meshes of eight-node quadrilaterals. The optimal convergence rate of two was not achieved in this case because element corrections are made only to satisfy first-order patch tests. Nevertheless, a convergence rate greater than unity was obtained.

The final example showed that improved accuracy can be achieved in certain instances by allowing the master boundary to have a greater number of nodes than the slave boundary. Standard practice commonly requires the master boundary to have fewer numbers of nodes. By relaxing this constraint, improved results were obtained as measured by the energy norm of the error and stresses along the shared boundary.

\section{Appendix}

Equations for $B_{j I}$ (see Eq. 6) of the elements shown in Figure 3 are provided here for completeness. One may obtain specific equations not shown by permuting the subscripts on the right hand sides of the equations and the subscript $I$ as described below. For notational convenience we adopt the conventions $x_{1 I}=x_{I}$ and $x_{2 I}=y_{I}$.

Three-node triangle:

$$
\begin{aligned}
& B_{1,1}=\left(y_{2}-y_{3}\right) / 2 \\
& B_{2,1}=\left(x_{3}-x_{2}\right) / 2
\end{aligned}
$$

Permutations: $1 \rightarrow 2 \rightarrow 3 \rightarrow 1$.

Four-node quadrilateral:

$$
\begin{aligned}
& B_{1,1}=\left(y_{2}-y_{4}\right) / 2 \\
& B_{2,1}=\left(x_{4}-x_{2}\right) / 2
\end{aligned}
$$

Permutations: $1 \rightarrow 2 \rightarrow 3 \rightarrow 4 \rightarrow 1$.

Six-node triangle:

$$
\begin{aligned}
& B_{1,1}=\left(y_{3}-y_{2}\right) / 6+2\left(y_{4}-y_{6}\right) / 3 \\
& B_{2,1}=\left(x_{2}-x_{3}\right) / 6+2\left(x_{6}-x_{4}\right) / 3 \\
& B_{1,4}=2\left(y_{2}-y_{1}\right) / 3 \\
& B_{2,4}=2\left(x_{1}-x_{2}\right) / 3
\end{aligned}
$$


Permutations: $1 \rightarrow 2 \rightarrow 3 \rightarrow 1$ and $4 \rightarrow 5 \rightarrow 6 \rightarrow 4$.

Eight-node quadrilateral:

$$
\begin{aligned}
& B_{1,1}=\left(y_{4}-y_{2}\right) / 6+2\left(y_{5}-y_{8}\right) / 3 \\
& B_{2,1}=\left(x_{2}-x_{4}\right) / 6+2\left(x_{8}-x_{5}\right) / 3 \\
& B_{5,1}=2\left(y_{2}-y_{1}\right) / 3 \\
& B_{5,2}=2\left(x_{1}-x_{2}\right) / 3
\end{aligned}
$$

Permutations: $1 \rightarrow 2 \rightarrow 3 \rightarrow 4 \rightarrow 1$ and $5 \rightarrow 6 \rightarrow 7 \rightarrow 8 \rightarrow 5$.

Nine-node triangle:

$$
\begin{aligned}
& B_{1,1}=7\left(y_{2}-y_{3}\right) / 80+57\left(y_{4}-y_{9}\right) / 80+3\left(y_{8}-y_{5}\right) / 10 \\
& B_{2,1}=7\left(x_{3}-x_{2}\right) / 80+57\left(x_{9}-x_{4}\right) / 80+3\left(x_{5}-x_{8}\right) / 10 \\
& B_{1,4}=\left(81 y_{5}-57 y_{1}\right) / 80-3 y_{2} / 10 \\
& B_{2,4}=\left(57 x_{1}-81 x_{5}\right) / 80+3 x_{2} / 10 \\
& B_{1,5}=\left(57 y_{2}-81 y_{4}\right) / 80+3 y_{1} / 10 \\
& B_{2,5}=\left(81 x_{4}-57 x_{2}\right) / 80-3 x_{1} / 10
\end{aligned}
$$

Permutations: $1 \rightarrow 2 \rightarrow 3 \rightarrow 1,4 \rightarrow 6 \rightarrow 8 \rightarrow 4$ and $5 \rightarrow 7 \rightarrow 9 \rightarrow 5$.

Twelve-node quadrilateral:

$$
\begin{aligned}
& B_{1,1}=7\left(y_{2}-y_{4}\right) / 80+3\left(y_{11}-y_{6}\right) / 10+57\left(y_{5}-y_{12}\right) / 80 \\
& B_{1,2}=7\left(x_{4}-x_{2}\right) / 80+3\left(x_{6}-x_{11}\right) / 10+57\left(x_{12}-x_{5}\right) / 80 \\
& B_{5,1}=\left(81 y_{6}-57 y_{1}\right) / 80-3 y_{2} / 10 \\
& B_{5,2}=\left(57 x_{1}-81 x_{6}\right) / 80+3 x_{2} / 10 \\
& B_{6,1}=\left(57 y_{2}-81 y_{5}\right) / 80+3 y_{1} / 10 \\
& B_{6,2}=\left(81 x_{5}-57 x_{2}\right) / 80-3 x_{1} / 10
\end{aligned}
$$

Permutations: $1 \rightarrow 2 \rightarrow 3 \rightarrow 4 \rightarrow 1,5 \rightarrow 7 \rightarrow 9 \rightarrow 11 \rightarrow 5$ and $6 \rightarrow 8 \rightarrow 10 \rightarrow 12 \rightarrow 6$.

We note that that Eqs. (58-81) are also applicable to elements with nodes internal to the element boundary such as the nine-node and sixteen-node Lagrange quadrilaterals. This is true because the coordinates of internal nodes do not affect element area. 


\section{References}

1. K. K. Ang and S. Valliappan, 'Mesh Grading Technique using Modified Isoparametric Shape Functions and its Application to Wave Propagation Problems,' International Journal for Numerical Methods in Engineering, 23, 331-348, (1986).

2. L. Quiroz and P. Beckers, 'Non-Conforming Mesh Gluing in the Finite Element Method,' International Journal for Numerical Methods in Engineering, 38, 2165-2184 (1995).

3. T. Y. Chang: A. F. Saleeb and S. C. Shyu, 'Finite Element Solutions of Two-Dimensional Contact Problems Based on a Consistent Mixed Formulation,' Computers and Structures. 27. 455-466 (1987).

4. O. C. Zienkiewicz and R. L. Taylor, The Finite Element Method, Vol. 1, 4th Ed., IIcGraw-Hill, New York, New York, 1989.

5. C. R. Dohrmann and S. W. Key, 'A Transition Element for Uniform Strain Hexahedral and Tetrahedral Finite Elements,' to appear in International Joumal for Numerical Methods in Engineering.

6. D. P. Flanagan and T. Belytschko, 'A Uniform Strain Hexahedron and Quadrilateral with Orthogonal Hourglass Control', International Journal for Numerical Methods in Engineering. 17, 679-706 (1981).

7. C. R. Dohrmann, S. W. Key, M. W. Heinstein and J. Jung, 'A Least Squares Approach for ('niform Strain Triangular and Tetrahedral Finite Elements', International Journal for Numerical Methods in Engineering, 42, 1181-1197 (1998).

8. S. P. Timoshenko and J. N. Goodier, Theory of Elasticity, 3rd Ed., McGraw-Hill, New York. . New York, 1970. 


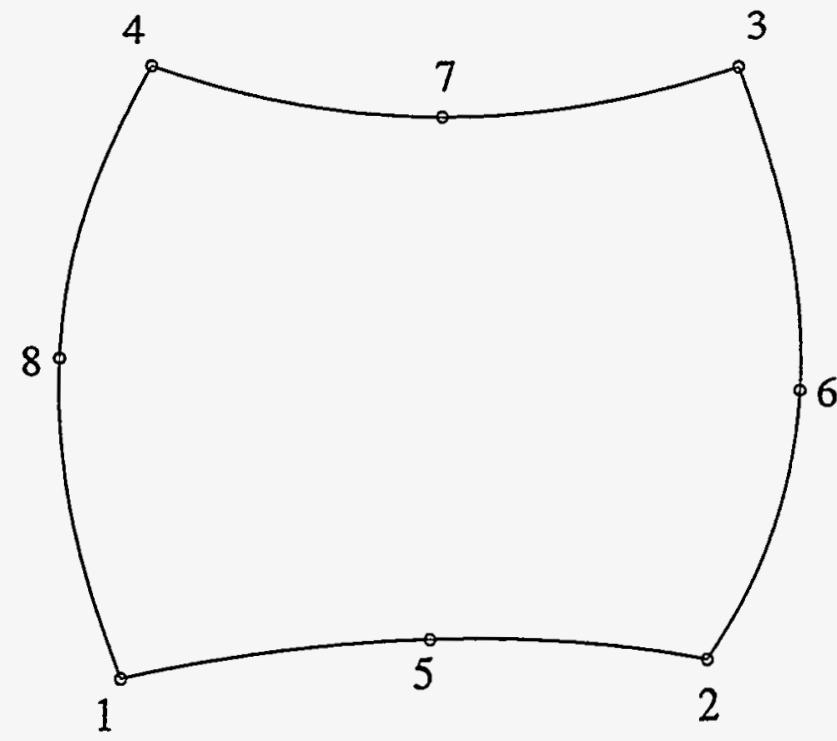

(a)

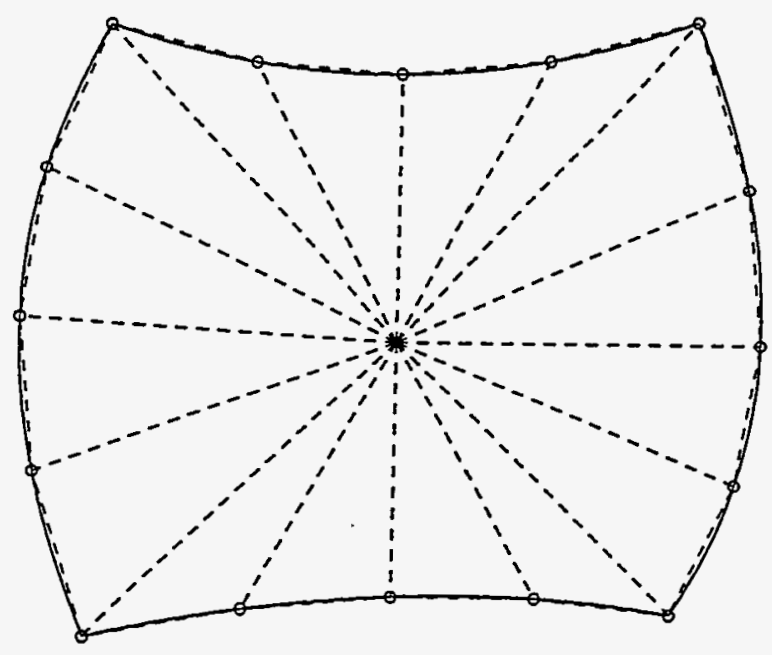

(b)

Figure 1: (a) Eight-node serendipity quadrilateral and (b) alternative geometric description of the element. 

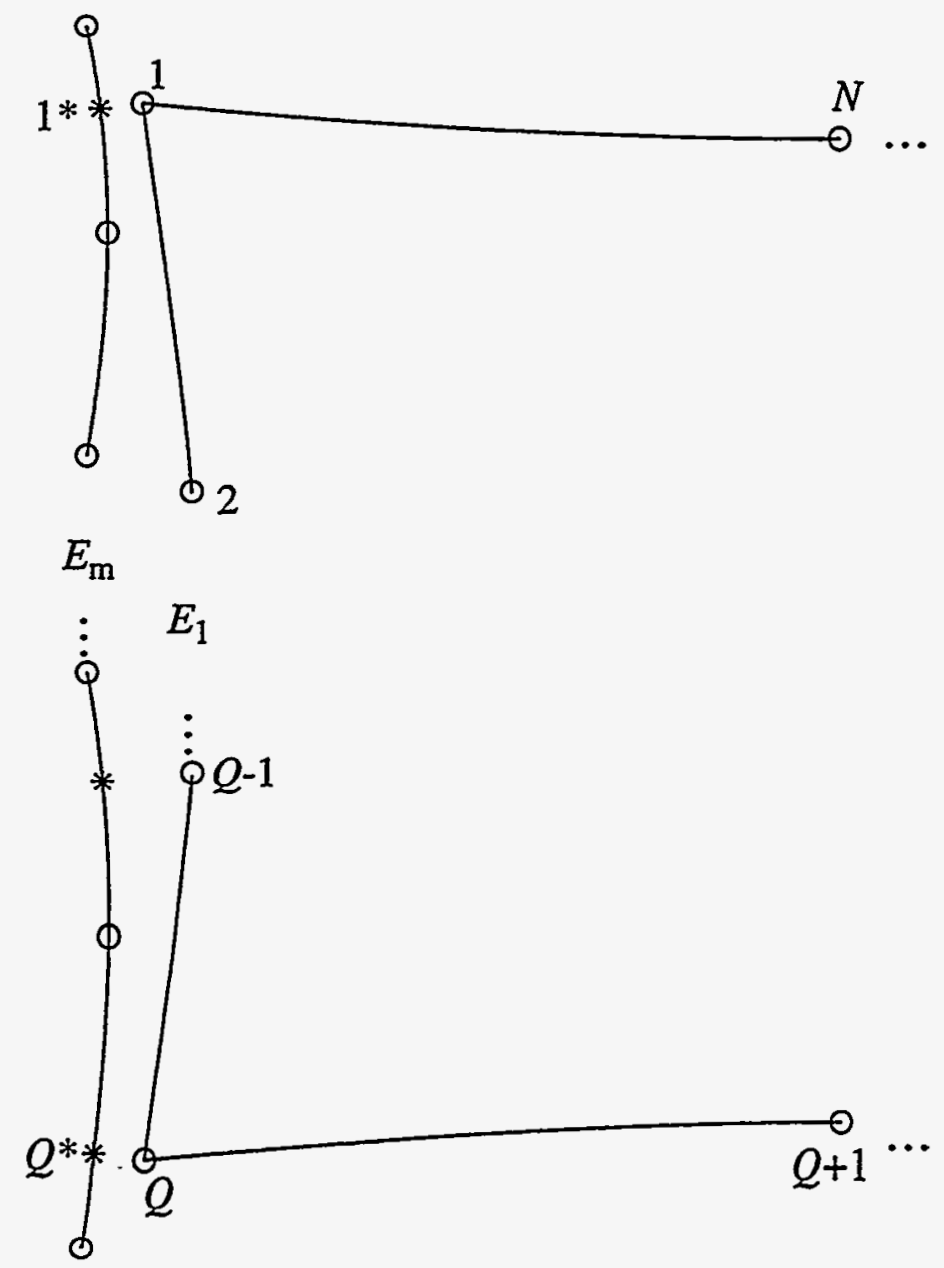

Figure 2: Sketch of meshes near element with an edge on the slave boundary. 


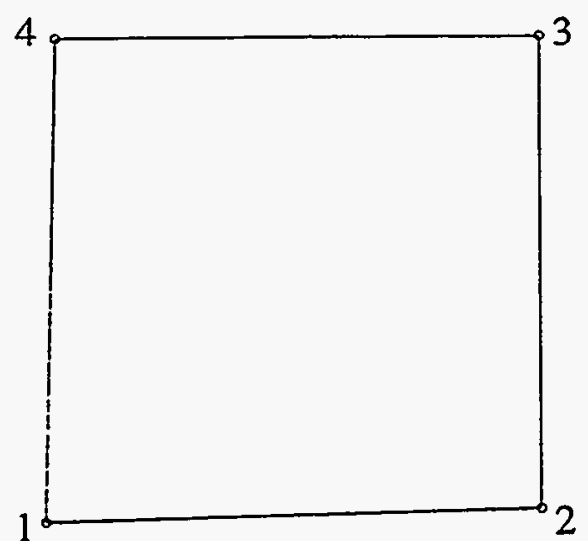

(a)

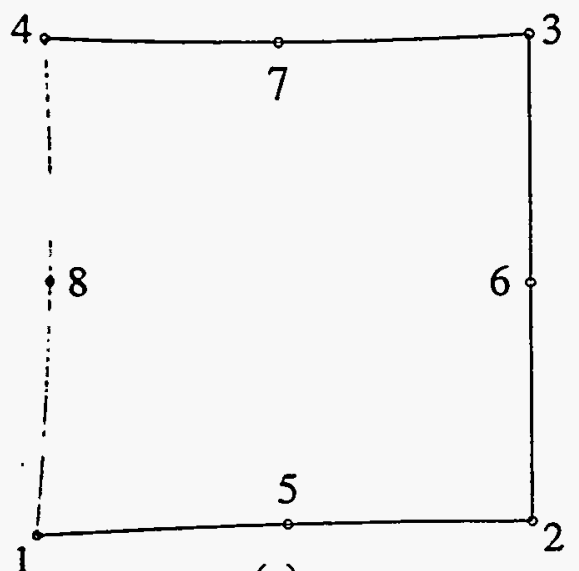

(c)

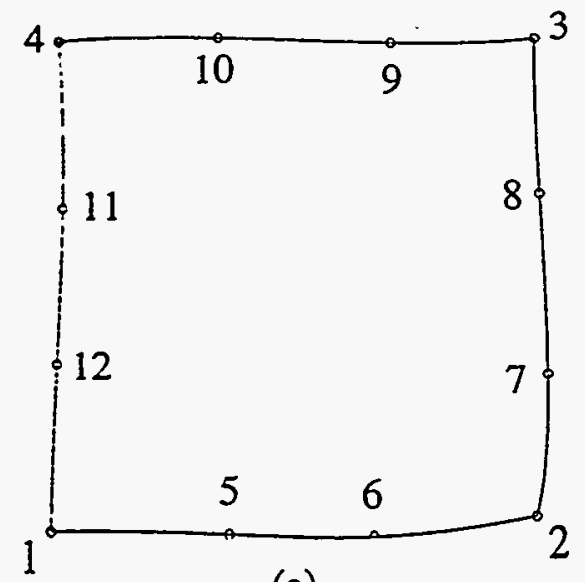

(e)

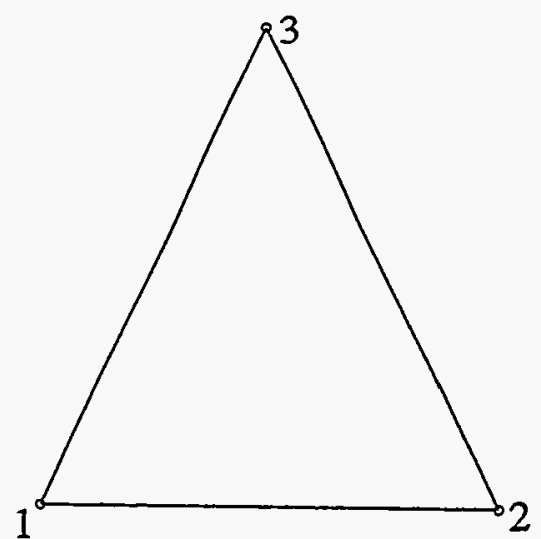

(b)

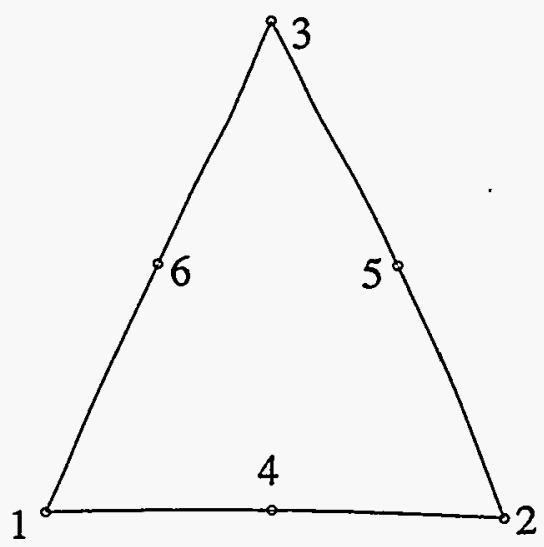

(d)

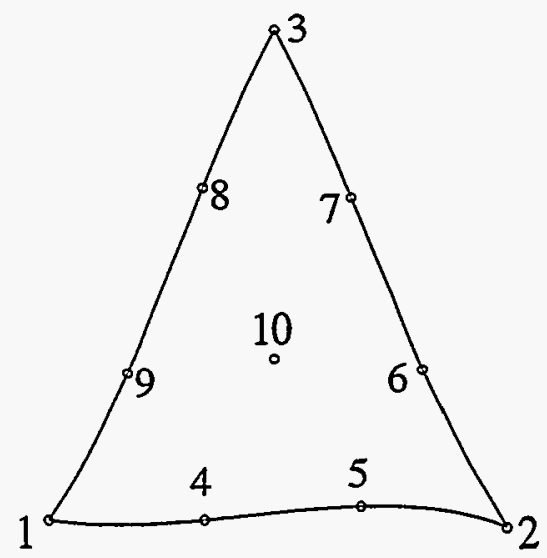

(f)

Figure 3: Element types considered in study: (a) four-node quadrilateral, (b) three-node triangle, (c) eightnode quadrilateral, (d) six-node triangle, (e) twelve-node quadrilateral and (f) ten-node triangle. 

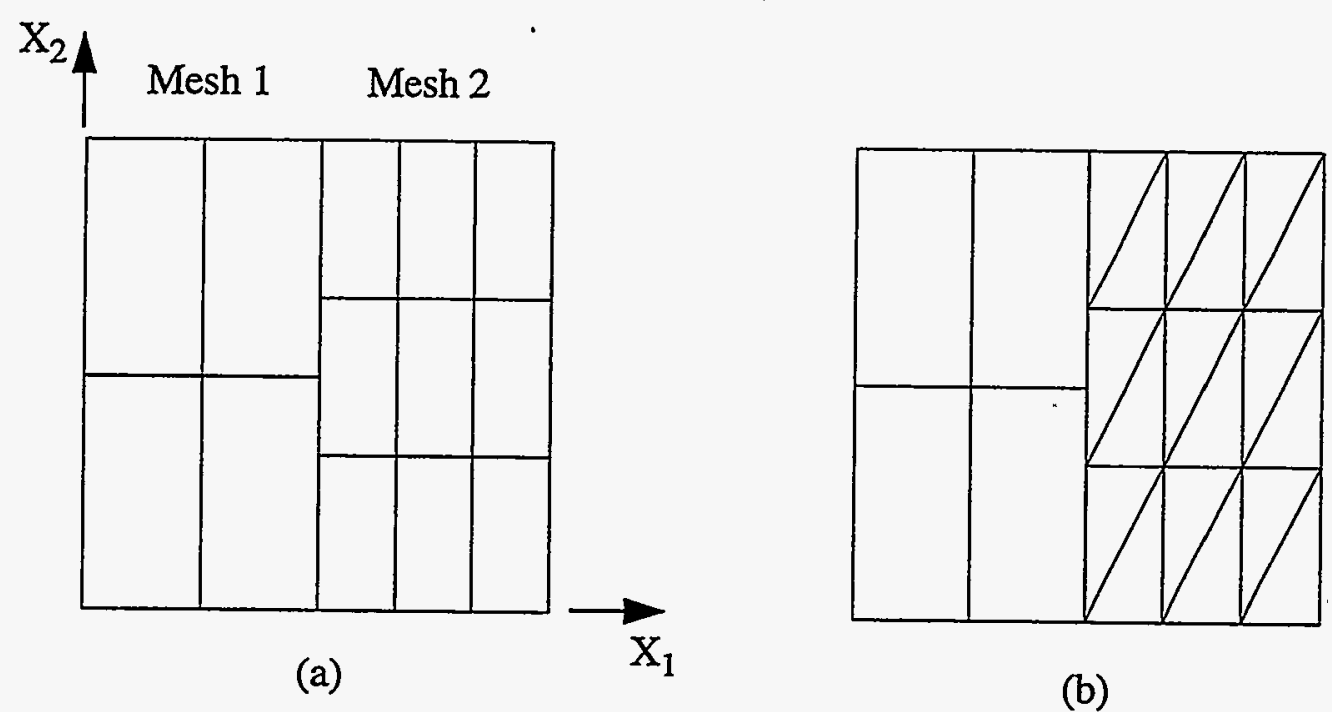

(b)
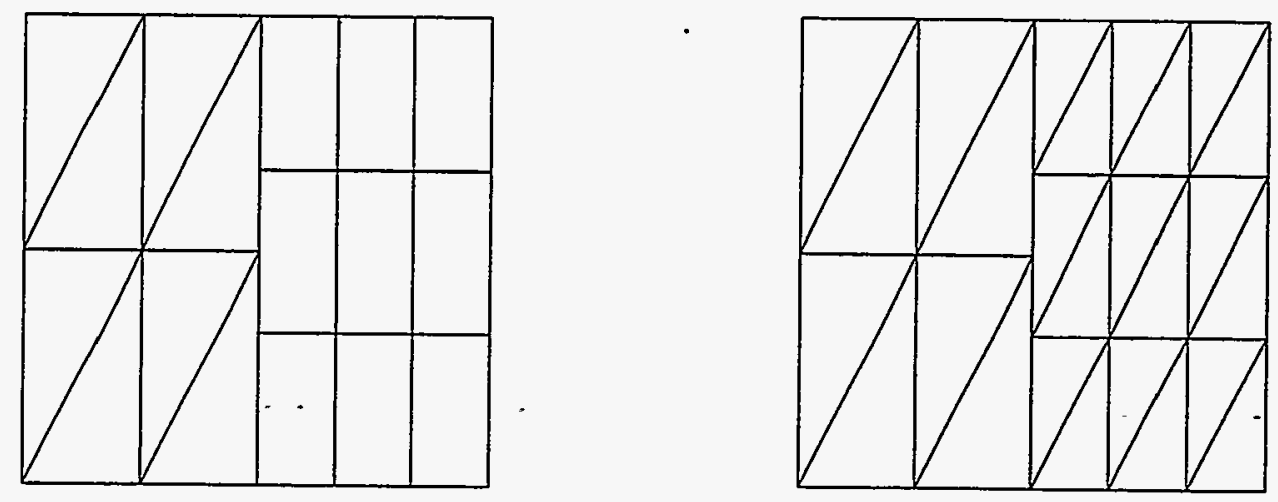

(c)

(d)

Figure 4: Mesh configurations (a) Q4Q4, (b) Q4T3, (c) T3Q4 and (d) T3T3 all with $n_{11}=n_{21}=2$ and $n_{12}=n_{22}=3$. 


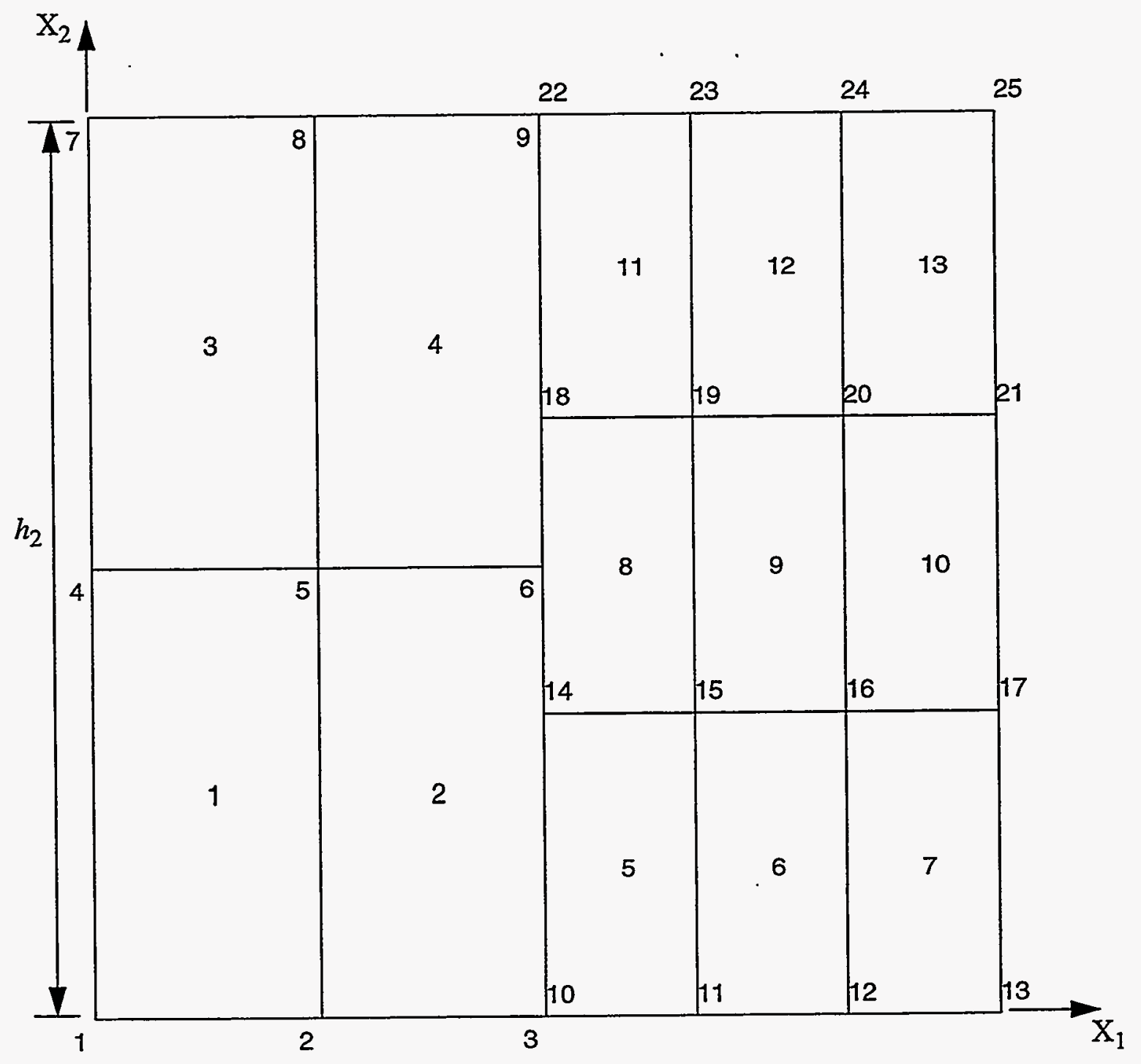

Figure 5: Mesh configuration Q4Q4 showing element and node numbers. 


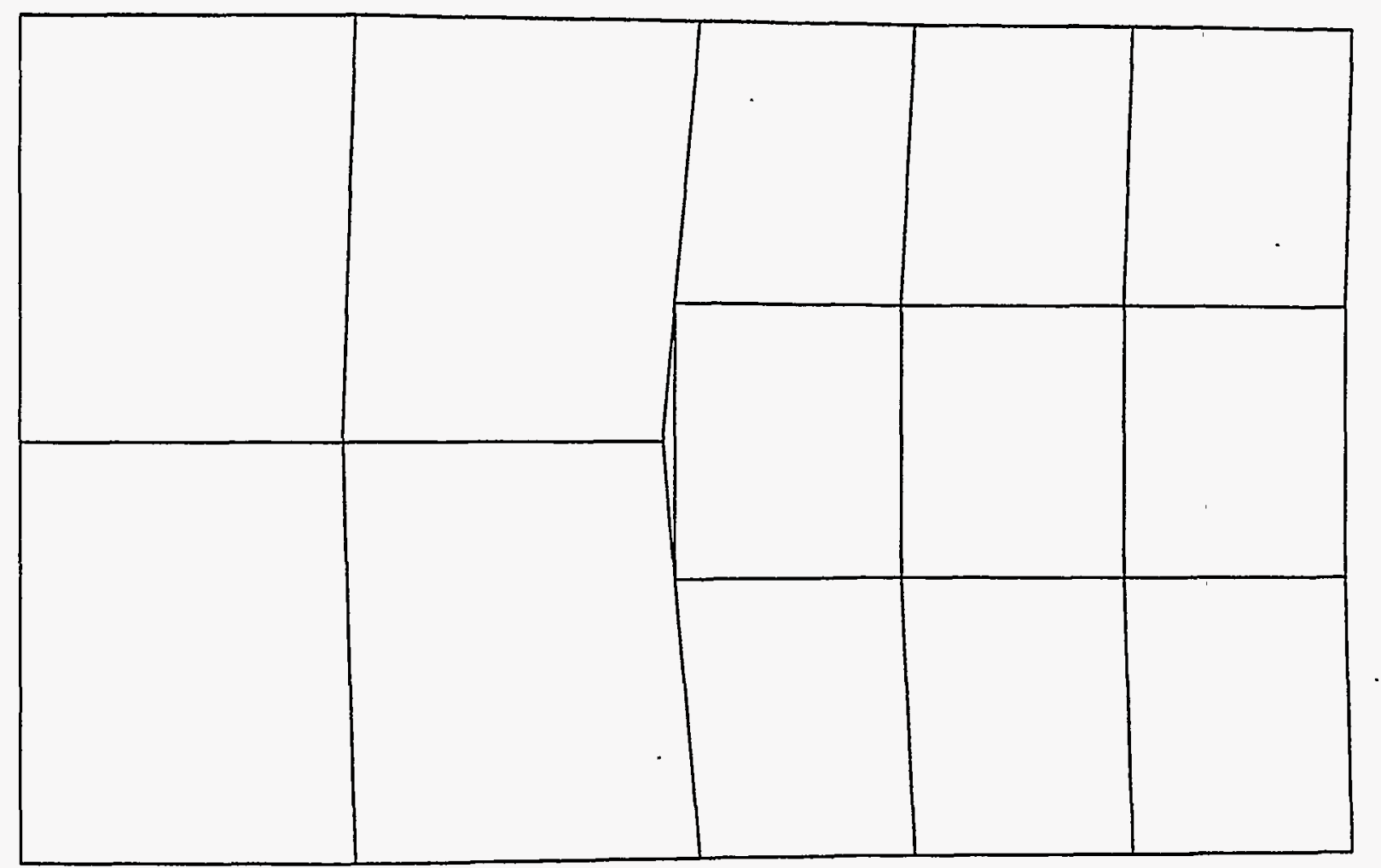

Figure 6: Example 3.1 displaced geometry (exaggerated) for mesh configuration Q4Q4 obtained using the standard master-slave approach. 


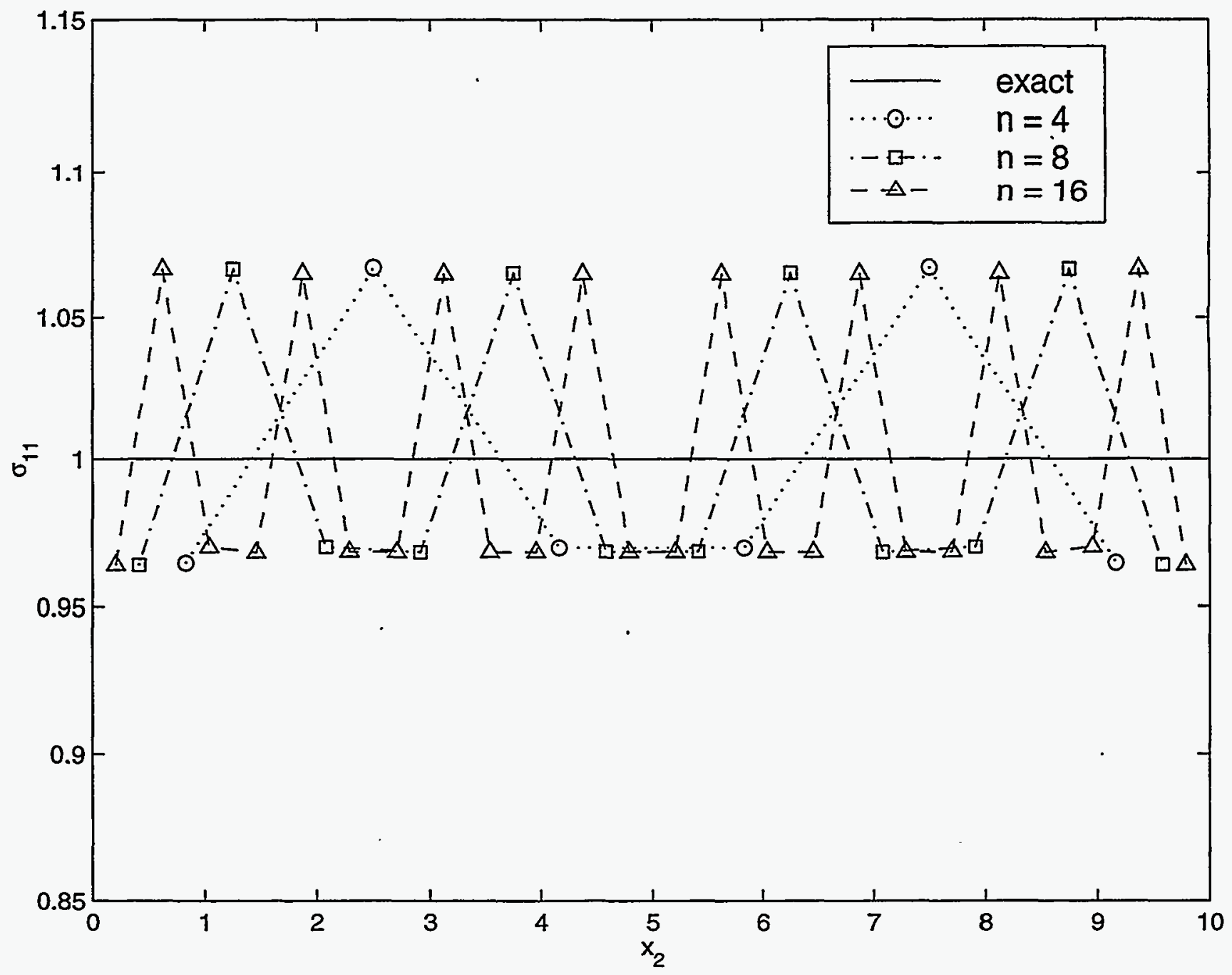

Figure 7: Axial stress $\sigma_{11}$ at centroids of elements with edges on the slave boundary. Results are presented for mesh configuration Q4Q4 using the standard master-slave approach. 


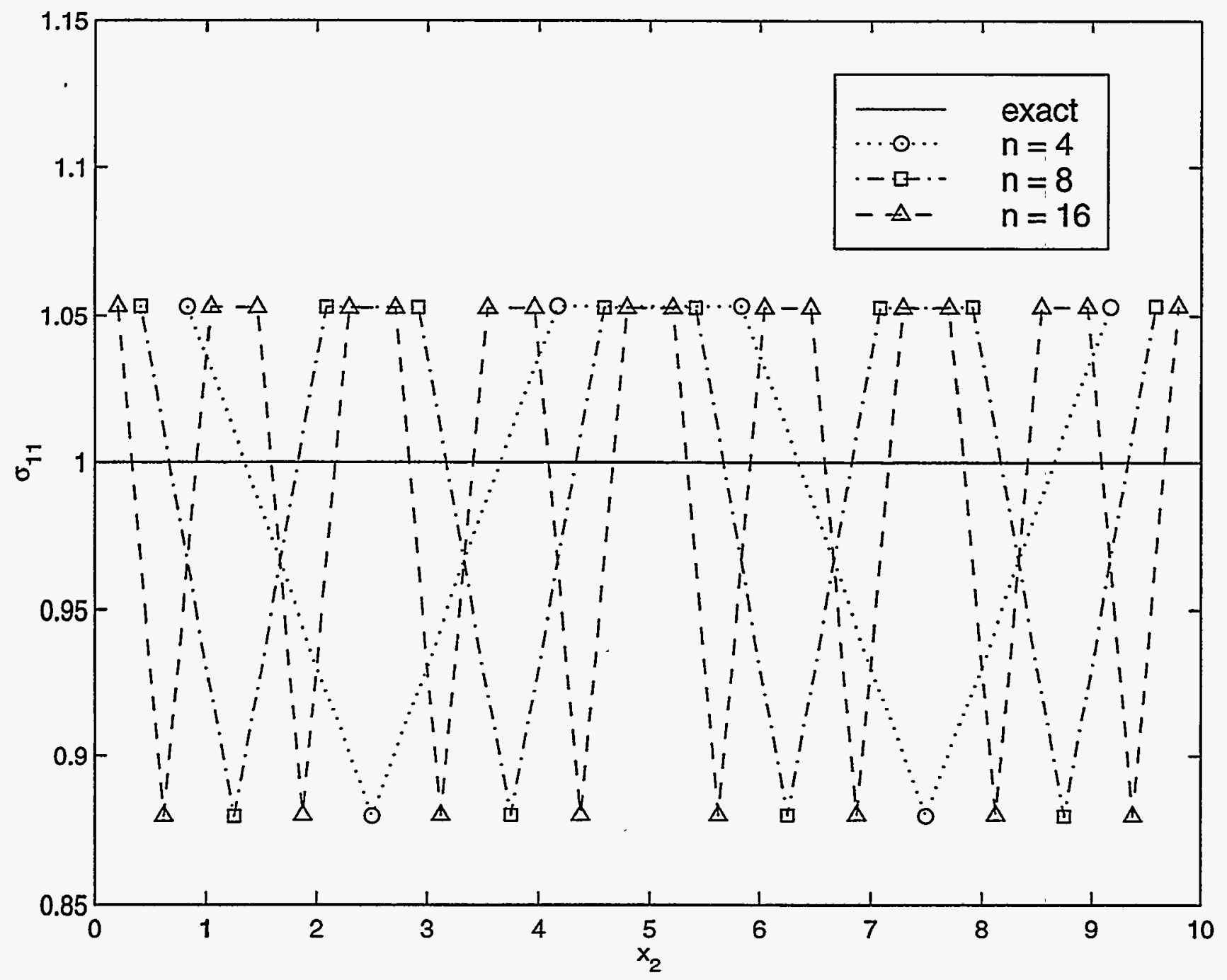

Figure 8: Axial stress $\sigma_{11}$ at centroids of elements with edges on the slave boundary. Results are presented for mesh configuration $\mathrm{Q} 8 \mathrm{Q} 8$ using the standard master-slave approach. 


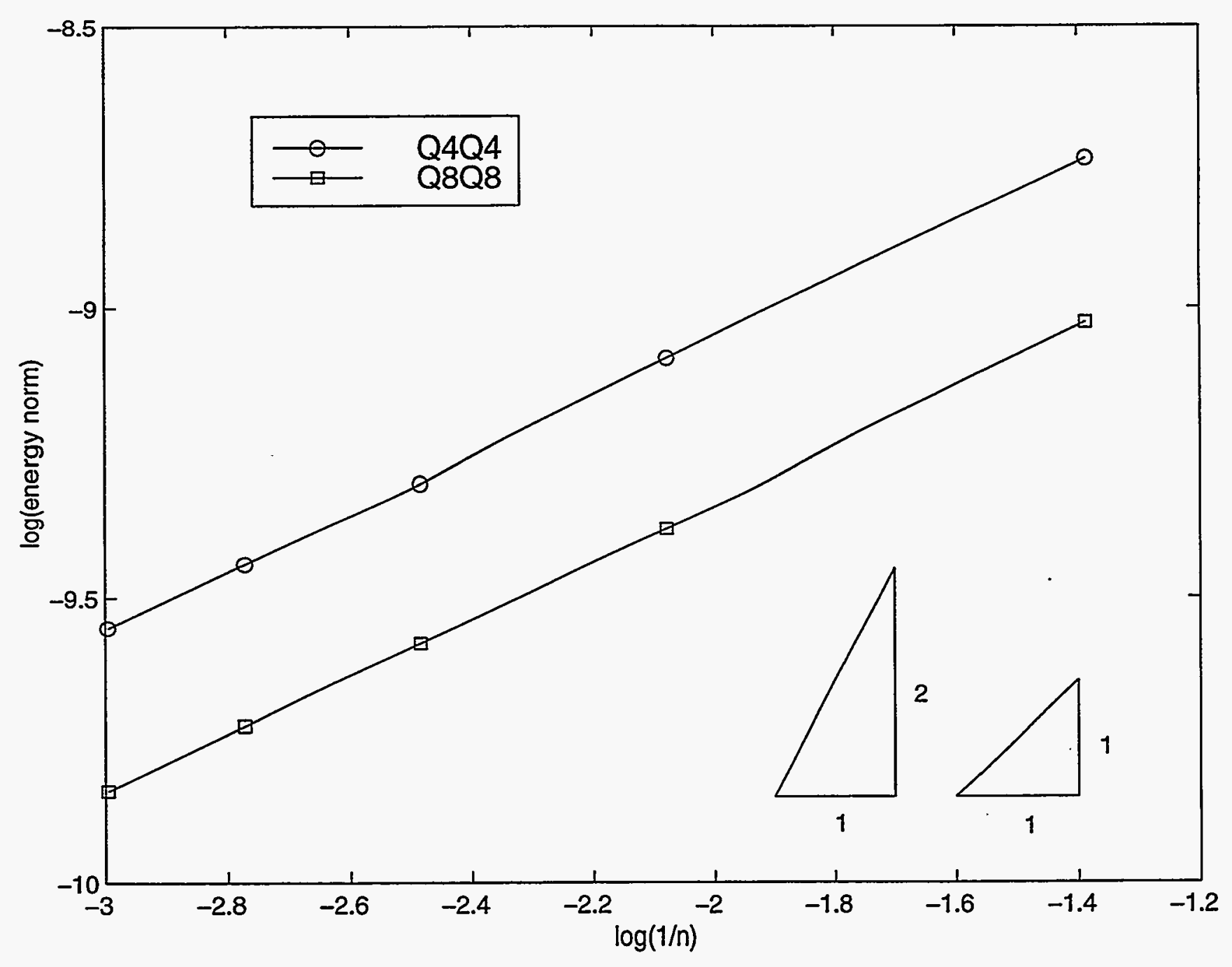

Figure 9: Energy norms of the error for Example 3.1 obtained using the standard master-slave approach. 


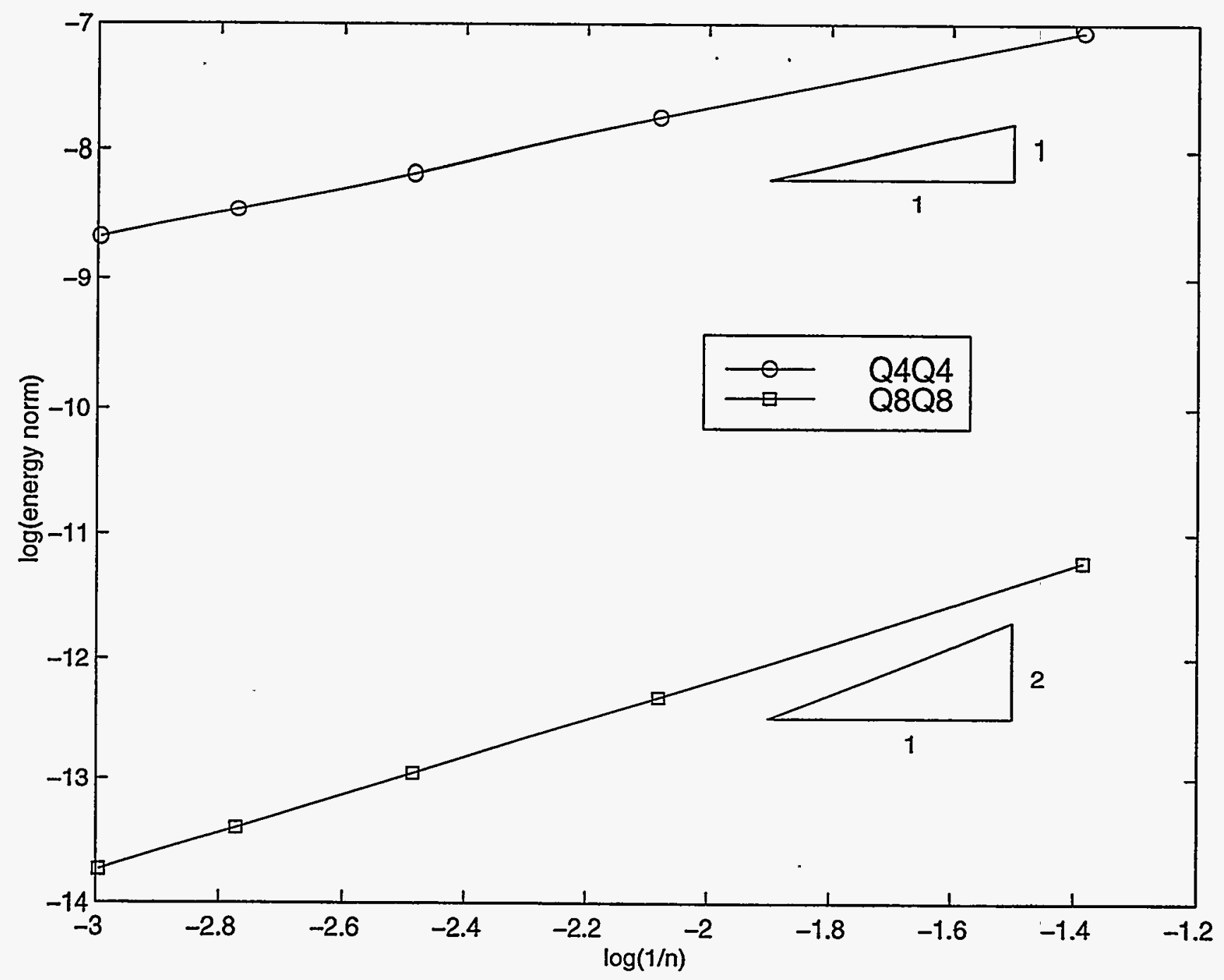

Figure 10: Energy norms of the error for Example 3.2 obtained using the present method. 


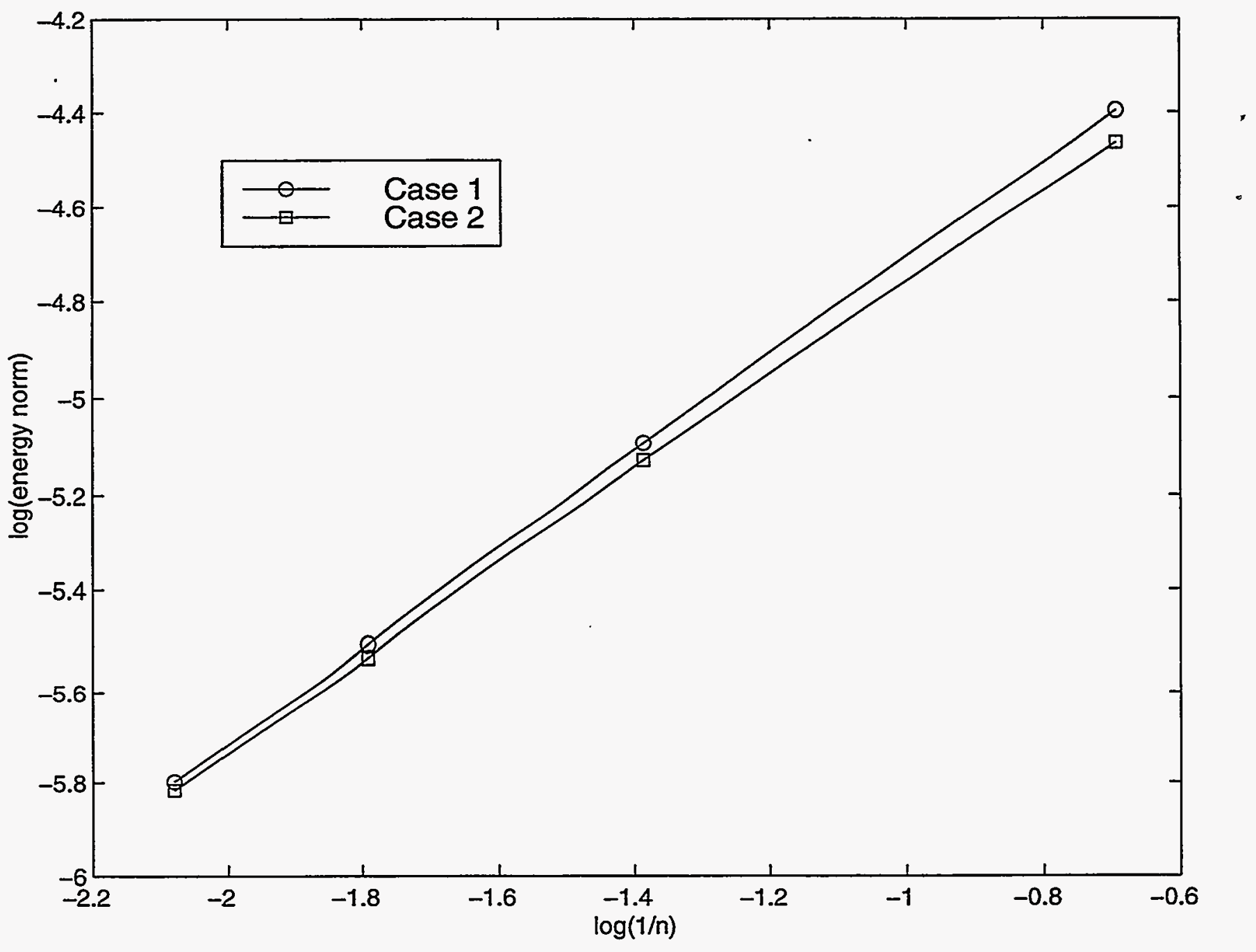

Figure 11: Energy norms of the error for Example 3.3 obtained using the present method for mesh configuration Q4Q4. Case $k$ refers to the problem with Mesh $k$ designated as master. 


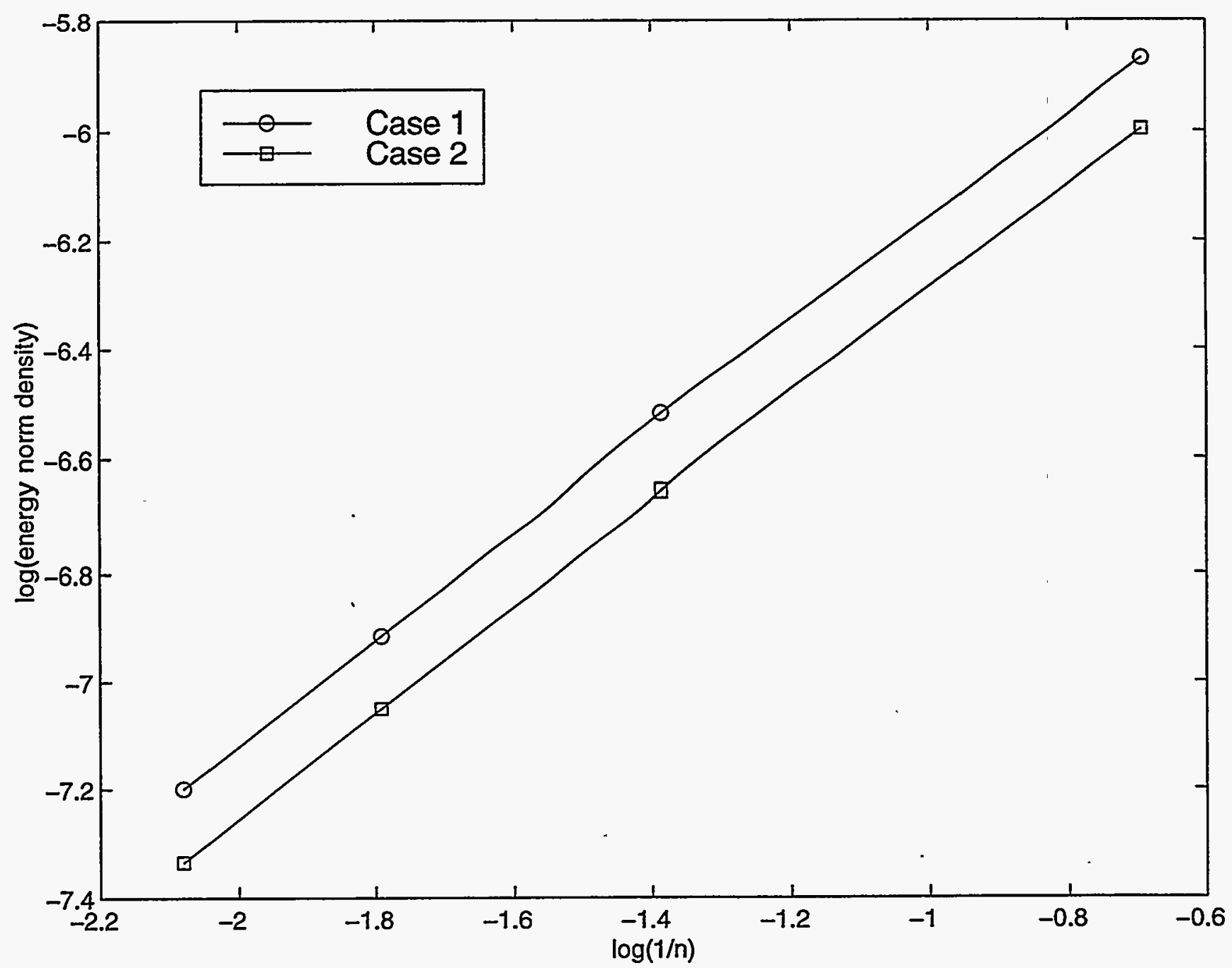

Figure 12: Energy norm densities of the error for Example 3.3 obtained using the present method for mesh configuration Q4Q4. Case $k$ refers to the problem with Mesh $k$ designated as master. 


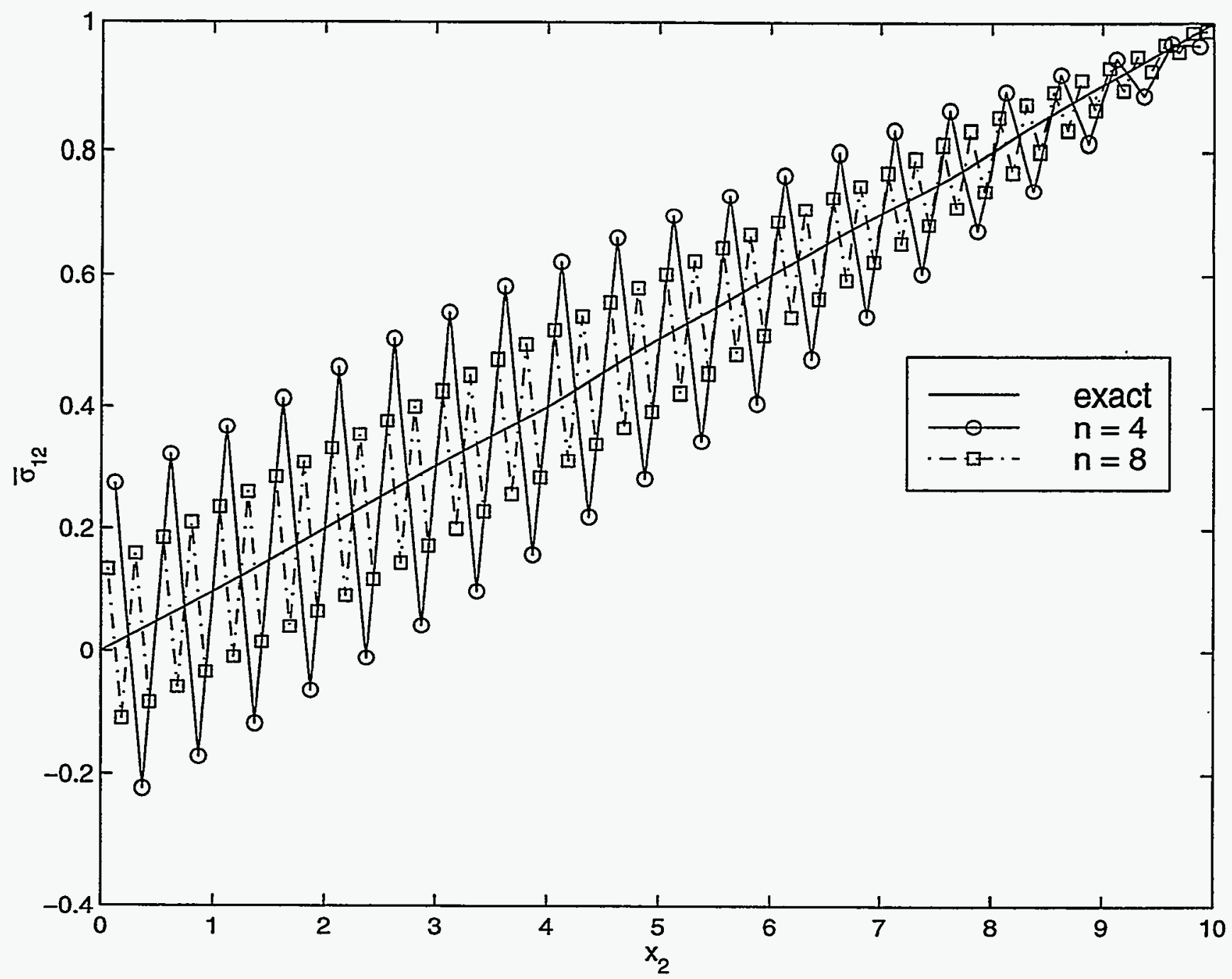

Figure 13: Normalized shear stress $\bar{\sigma}_{12}$ at centroids of elements with edges on the slave boundary for mesh configuration Q4Q4 (Case 1). 


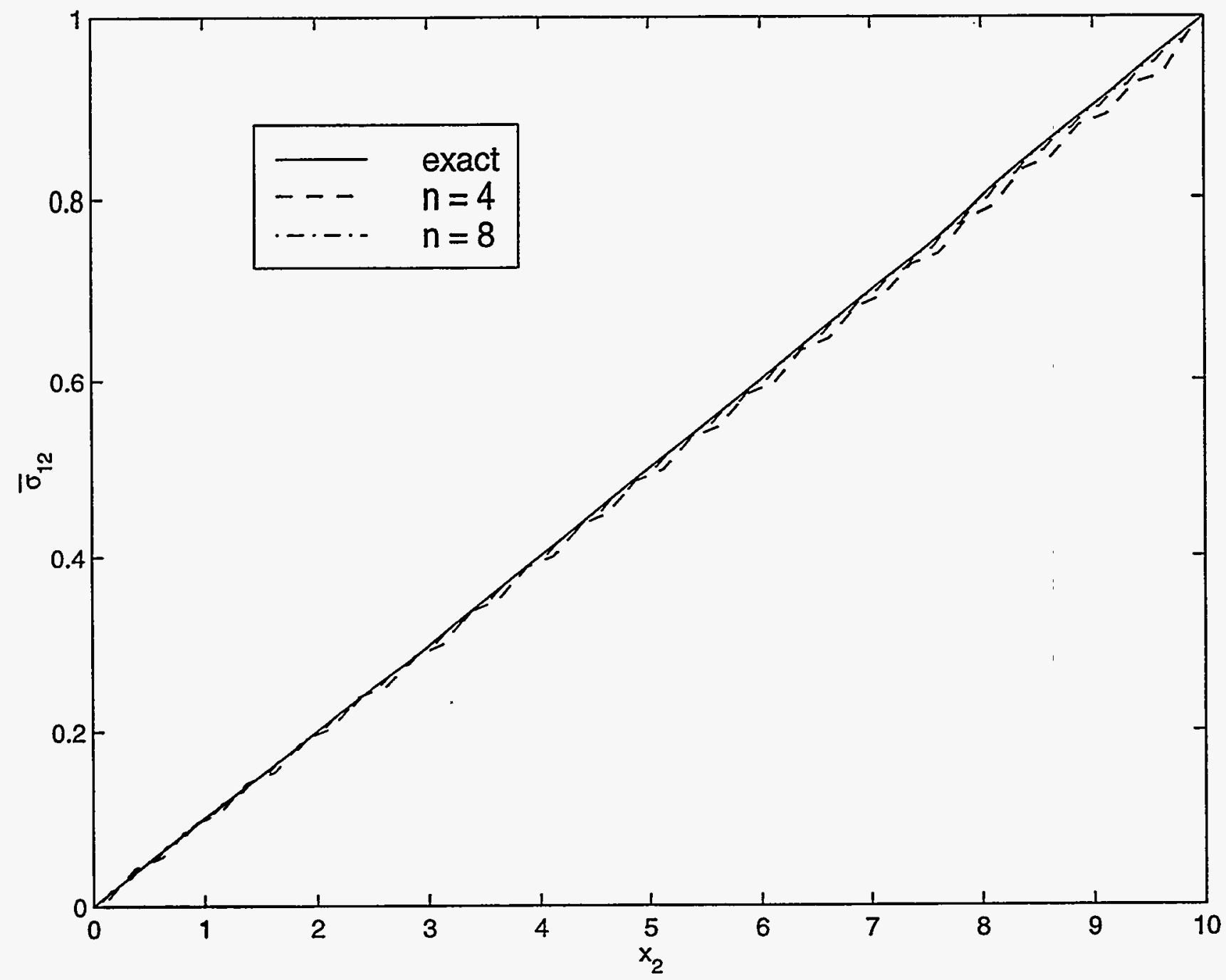

Figure 14: Normalized shear stress $\bar{\sigma}_{12}$ at centroids of elements with edges on the slave boundary for mesh configuration Q4Q4 (Case 2). 


\title{
A Method for Connecting Dissimilar Finite Element Meshes in Three Dimensions ${ }^{1}$
}

\author{
C. R. Dohrmann ${ }^{2}$ \\ S. W. $\mathrm{Key}^{3}$ \\ M. W. Heinstein ${ }^{3}$
}

\begin{abstract}
A method is presented for connecting dissimilar finite element meshes in three dimensions. The method combines the concept of master and slave surfaces with the uniform strain approach for finite elements. By modifying the boundaries of elements on the slave surface, corrections are made to element formulations such that first-order patch tests are passed. The method can be used to connect meshes which use different element types. In addition, master and slave surfaces can be designated independently of relative mesh resolutions. Example problems in three-dimensional linear elasticity are presented.
\end{abstract}

Key Words. Finite elements, connected meshes, uniform strain, contact.

\footnotetext{
${ }^{1}$ Sandia is a multiprogram laboratory operated by Sandia Corporation, a Lockheed Martin Company, for the United States Department of Energy under Contract DE-AL04-94AL8500.

${ }^{2}$ Structural Dynamics Department, Sandia National Laboratories, MS 0439, Albuquerque, New Mexico 87185-0439, email: crdohrm@sandia.gov, phone: (505) 844-8058, fax: (505) 844-9297.

${ }^{3}$ Engineering and Manufacturing Mechanics Department, Sandia National Laboratories, MS 0443, Albuquerque, New Mexico 87185-0443.
} 


\section{Introduction}

In order to perform a finite element analysis, one may be required to connect two meshes at a shared boundary. Such requirements are common when assembling system models from separate subsystem models. One approach to connecting the meshes requires that both meshes have the same number of nodes, the same nodal coordinates, and the same interpolation functions at the shared boundary. If these requirements are met, then the two meshes can be connected simply by equating the degrees of freedom of corresponding nodes at the shared boundary. As might be expected, connecting meshes in this manner often requires a significant amount of time and effort in mesh generation.

An alternative to such an approach is to use the concept of "tied contact" to connect the meshes. With this concept, one of the connecting mesh surfaces is designated as the master surface and the other as the slave surface. For problems in solid mechanics, the meshes are connected by constraining nodes on the slave surface to specific points on the master surface at all times. Although this approach is appealing because of its simplicity, overlaps and gaps may develop between the two meshes either because of non-planar initial geometry or nonuniform displacements. For example, a node on the master surface may either penetrate or pull away from the slave surface during deformation even though the slave node constraints are all satisfied. As a result, displacement continuity may not hold at all locations on the master-slave interface.

Several methods currently exist for connecting finite elements or meshes of elements. Mesh grading approaches allow two or more finer elements to abut the edge of a neighboring coarser element [1]. Although such approaches generate conforming element boundaries, they are not applicable to the general problem of connecting two dissimilar meshes. Other methods [2-3] for connecting meshes based on constraint equations or Lagrange multiplier approaches are applicable to a much broader class of problems, but they generally do not ensure that mesh boundaries conform during deformation. Finite element approaches developed specifically for contact problems can also be used to connect meshes. These [4] include: (i) Lagrange multiplier methods; (ii) penalty methods; and (iii) mixed methods. Many of these methods are based in part on the master-slave concept.

Regardless of the method used to connect two meshes, it is important to address the issues related to continuity at the mesh boundaries. One such issue is the first-order patch test [5]. In general, meshes that are connected using existing methods based on constraint equations or penalty functions alone fail the patch test. A general method for connecting finite element meshes in two dimensions that passes the patch test was developed recently by the authors [6]. This study investigates an extension of that method to three dimensions. The basic idea is to redefine the boundaries of elements on the slave surface to achieve a conforming connection with the master surface. The same idea was used recently at the element level to obtain a conforming transition between hexahedral and tetrahedral elements [7].

The present method combines the master-slave concept with the uniform strain approach for finite elements [8]. As with the standard master-slave approach, nodes on the slave surface are constrained to the master surface. In addition, the boundaries and formulations 
of elements on the slave surface are modified to ensure that first-order patch tests are passed. Consequently, results obtained using the method converge with mesh refinement.

A useful feature of the method is the freedom to designate the master and slave surfaces independently of the resolutions of the two meshes. Standard practice commonly requires the surface designated as the master to have fewer numbers of nodes than the slave surface. The present method allows one to specify either of the mesh boundaries as master while still satisfying the patch test. It is shown in Section 3 that improved accuracy can be achieved in certain instances by allowing the master surface to have the greater number of nodes. Thus, there may be a preferred choice for the master surface in certain cases. Methods of mesh refinement based on adaptive subdivision of existing elements may also benefit from the method. For example, kinematic constraints on improper nodes could be removed while preserving displacement continuity between adjacent elements.

Details of the method are presented in the following section. The presentation includes a discussion of the uniform strain approach and the geometric concepts upon which the method is based. Example problems in three-dimensional linear elasticity are presented in Section 3. These examples highlight the various capabilities of the method. Comparisons made with the standard master-slave approach demonstrate the superior performance of the method.

\section{Formulation}

Consider a generic finite element in three dimensions with nodal coordinates $x_{i I}$ and nodal displacements $u_{i I}$ for $i=1,2,3$ and $I=1, \ldots, N$. The spatial coordinates and displacements of a point in the global coordinate direction $\mathbf{e}_{i}$ are denoted by $x_{i}$ and $u_{i}$, respectively. For isoparametric elements, the same interpolation functions are used for the coordinates and displacements. That is,

$$
\begin{aligned}
& x_{i}=x_{i I} \phi_{I}\left(\eta_{1}, \eta_{2}, \eta_{3}\right) \\
& u_{i}=u_{i I} \phi_{I}\left(\eta_{1}, \eta_{2}, \eta_{3}\right)
\end{aligned}
$$

where $\phi_{I}$ is the shape function of node $I$ and $\left(\eta_{1}, \eta_{2}, \eta_{3}\right)$ are isoparametric coordinates. A summation over all possible values of repeated indices in Eqs. (1-2) and elsewhere is implied unless noted otherwise.

The Jacobian determinant $J$ of the element is defined as

$$
J=\operatorname{det}\left[\begin{array}{lll}
\partial x_{1} / \partial \eta_{1} & \partial x_{2} / \partial \eta_{1} & \partial x_{3} / \partial \eta_{1} \\
\partial x_{1} / \partial \eta_{2} & \partial x_{2} / \partial \eta_{2} & \partial x_{3} / \partial \eta_{2} \\
\partial x_{1} / \partial \eta_{3} & \partial x_{2} / \partial \eta_{3} & \partial x_{3} / \partial \eta_{3}
\end{array}\right]
$$

The volume $V$ of the element can be expressed in terms of $J$ by

$$
V=\int_{V_{\eta}} J d V
$$

where $V_{\eta}$ is the volume of integration of the element in the isoparametric coordinate system. 
It is assumed that $V$ is a homogeneous function of the nodal coordinates. It is also assumed that a linear displacement field can be expressed exactly in terms of the shape functions. Under these conditions, the uniform strain approach of Ref. 8 states that the nodal forces $f_{i I}$ associated with element stresses are given by

$$
f_{i I}=\sigma_{i j} B_{j I}
$$

where $\sigma_{i j}$ are components of the Cauchy stress tensor (assumed constant throughout the element), and

$$
B_{j I}=\frac{\partial V}{\partial x_{j I}}
$$

In addition, one has

$$
V=x_{j I} B_{j I} \quad \text { for } \quad j=1,2,3
$$

where there is no summation over the index $j$ in Eq. (7).

Closed-form expressions for $B_{j I}$ are presented in Ref. 8 for the 8-node hexahedron. Similar expressions can be derived for other element types, but they are quite lengthy for higherorder elements. As an alternative to deriving closed-form expressions for specific element types, one can use Gauss quadrature to determine $B_{j I}$ for any isoparametric element in a systematic manner.

By substituting Eqs. (1), (3) and (4) into Eq. (6), one finds that the functions $g_{j I}$ used by the quadrature rule to evaluate $B_{j I}$ are given by

$$
\begin{aligned}
& g_{1 I}=\phi_{I, 1}\left(x_{2,2} x_{3,3}-x_{3,2} x_{2,3}\right)+\phi_{I, 2}\left(x_{2,3} x_{3,1}-x_{3,3} x_{2,1}\right)+\phi_{I, 3}\left(x_{2,1} x_{3,2}-x_{3,1} x_{2,2}\right) \\
& g_{2 I}=\phi_{I, 1}\left(x_{3,2} x_{1,3}-x_{1,2} x_{3,3}\right)+\phi_{I, 2}\left(x_{3,3} x_{1,1}-x_{1,3} x_{3,1}\right)+\phi_{I, 3}\left(x_{3,1} x_{1,2}-x_{1,1} x_{3,2}\right) \\
& g_{3 I}=\phi_{I, 1}\left(x_{1,2} x_{2,3}-x_{2,2} x_{1,3}\right)+\phi_{I, 2}\left(x_{1,3} x_{2,1}-x_{2,3} x_{1,1}\right)+\phi_{I, 3}\left(x_{1,1} x_{2,2}-x_{2,1} x_{1,2}\right)
\end{aligned}
$$

where

$$
\begin{gathered}
\phi_{I, j}=\partial \phi_{I} / \partial \eta_{j} \\
x_{i, j}=\partial x_{i} / \eta_{j}=x_{i I}\left(\partial \phi_{I} / \partial \eta_{j}\right)
\end{gathered}
$$

and $g_{j I}$ is evaluated at each of the quadrature points. Exact values of $B_{j I}$ can be obtained using 2-point Gauss quadrature in three dimensions (8 quadrature points total) for the 8node hexahedron. For the 20-node serendipity or 27-node Lagrange hexahedron, 3-point Gauss quadrature in three dimensions (27 quadrature points total) is required. Exact values of $B_{j I}$ for the 4-node linear tetrahedron can be obtained using a 1-point quadrature rule for tetrahedral domains while the 10-node quadratic tetrahedron requires a 5-point quadrature rule. Quadrature rules for integration over tetrahedral domains are available in Ref. 5.

Following the development in Ref. 8, one can show that

$$
\int_{\Omega} \frac{\partial \phi_{I}}{\partial x_{j}} d V=B_{j I}
$$


where $\Omega$ is the domain of the element in the global coordinate system. Based on Eq. (13), the uniform strain $\epsilon^{u}$ of the element is expressed in terms of nodal displacements as

$$
\epsilon^{u}=C u
$$

where

$$
\begin{gathered}
\epsilon^{u}=\left[\begin{array}{lccccccccc}
\epsilon_{11}^{u} & \epsilon_{22}^{u} & \epsilon_{33}^{u} & \gamma_{12}^{u} & \gamma_{23}^{u} & \gamma_{31}^{u}
\end{array}\right] \\
C=\frac{1}{V}\left[\begin{array}{cccccccccc}
B_{11} & 0 & 0 & B_{12} & 0 & 0 & \cdots & B_{1 N} & 0 & 0 \\
0 & B_{21} & 0 & 0 & B_{22} & 0 & \cdots & 0 & B_{2 N} & 0 \\
0 & 0 & B_{31} & 0 & 0 & B_{32} & \cdots & 0 & 0 & B_{3 N} \\
B_{21} & B_{11} & 0 & B_{22} & B_{12} & 0 & \cdots & B_{2 N} & B_{1 N} & 0 \\
0 & B_{31} & B_{21} & 0 & B_{32} & B_{22} & \cdots & 0 & B_{3 N} & B_{2 N} \\
B_{31} & 0 & B_{11} & B_{32} & 0 & B_{21} & \cdots & B_{3 N} & 0 & B_{1 N}
\end{array}\right]
\end{gathered}
$$

and

$$
u=\left[\begin{array}{llllllllll}
u_{11} & u_{21} & u_{31} & u_{12} & u_{22} & u_{32} & \cdots & u_{1 N} & u_{2 N} & u_{3 N}
\end{array}\right]^{T}
$$

Elements based on the uniform strain approach have the appealing feature that they pass first-order patch tests.

Boundaries of three-dimensional elements are defined either by planar or curved faces. Elements with interpolation functions that vary linearly, e.g. the 4-node tetrahedron, have planar faces. In contrast, elements with higher-order interpolation functions, e.g. the 8-node hexahedron and 10-node tetrahedron, generally have curved faces. That being the case, it may not be obvious how to connect two meshes of elements which use different orders of interpolation along their boundaries.

Difficulties can arise using the standard master-slave approach even if the boundaries of both meshes are defined by planar faces. As was mentioned previously, even though the slave nodes stay attached to the master surface, there may not be any constraints to keep a node on the master boundary from penetrating or pulling away from the slave boundary. Such problems are addressed with the present method by requiring the faces of elements on the slave boundary to always conform to the master boundary. In order to explain how this is done, some preliminary geometric concepts are introduced first.

Notice from Eqs. (6), (14) and (16) that the relationship between strain and displacement for a uniform strain element is defined completely by its volume. Consequently, the uniform strain characteristics of two elements are identical if the expressions for their volumes are the same. This fact is important because it allows one to consider alternative interpolation functions for elements with faces on the master and slave surfaces. By doing so, one can interpret the present method as an approach for generating "conforming" finite elements at the shared boundary by carefully accounting for the volume (positive or negative) that exists due to an imperfect match between the two meshes both initially and during deformation.

Consider an 8-node hexahedral element whose six faces are not necessarily planar. Each point on a face of the element is associated with specific values of two isoparametric coordinates. Both the spatial coordinates and displacements of the point are linear functions of 
the coordinates and displacements of the four nodes defining the face. The specific forms of these relationships are obtained by setting either $\eta_{1}, \eta_{2}$ or $\eta_{3}$ equal to one of its bounding values in Eqs. (1-2).

Consider now an alternative element in which each face of the original 8-node hexahedron is triangulated with $n_{t}$ facets. Each vertex of a triangular facet intersects one of the curved faces of the hexahedron. A center node $c$ is introduced in the interior of the element. Although the precise location of $c$ is not important, its coordinates can be expressed in terms of those of the hexahedron as

$$
x_{i c}=\sum_{I=1}^{8} x_{i I} / 8
$$

The center node along with the three vertices of each triangular facet form the vertices of a 4-node tetrahedron. Thus, the domain of the hexahedron can be divided into $6 n_{t}$ tetrahedral regions. Within each of these regions the interpolation functions are linear. In other words, the displacement of a point in a tetrahedral region is determined by its location and the displacements of the four nodes defining the tetrahedron. One may approximate the boundary of the original hexahedron to any level of accuracy by increasing the number of triangular facets.

Although the two elements described in the previous paragraphs are significantly different, their uniform strain characteristics are approximately the same. In the limit as $n_{t}$ approaches infinity, the uniform strain characteristics of the two elements are identical. By viewing all the element faces on the master and slave surfaces as connected triangular facets, one can develop a systematic method for connecting the two meshes that passes first-order patch tests. We note that the alternative element satisfies the basic assumptions of the uniform strain approach. That is, the element volume is a homogeneous function of the nodal coordinates and a linear displacement field can be expressed exactly in terms of the interpolation functions.

We are now in a position to present the method for modifying elements with faces on the slave boundary. Changes to elements with faces on the master boundary are not required. The concept of alternative piecewise-linear interpolation functions was introduced in the previous paragraphs to facilitate interpretation of the method as a means for generating conforming elements at the master-slave interface. These alternative interpolation functions are never used explicitly to modify the element formulations.

Figure 1 depicts the projection of an element face $F_{1}$ of the slave surface onto the master surface. The larger filled circles designate nodes on the slave surface constrained to the master surface. Smaller filled circles designate nodes on the master surface. Circles that are not filled designate the projections of slave element edges onto master element edges.

Although there are several options for projecting slave element entities onto the master surface, we opted for the following in this study. Nodes on the slave surface that are initially off the master surface are repositioned to specific points on the master surface based on a minimum distance criterion. That is, a node on the slave surface is moved and constrained to the nearest point on the master surface. For each element face of the slave surface, one 
can define a normal direction at the center of the face. If an element edge of the slave surface is shared by two elements, the normal direction for the edge is defined as the average of the two elements sharing the edge. Otherwise, the normal direction is chosen as that of the single element containing the edge. A plane is constructed which contains two nodes of the slave element edge and has a normal in the direction of the cross product of the element edge and the element edge normal. The projection of the slave element edge onto a master element edge is simply the intersection of this plane with the master element edge.

Let $P$ denote the element face of the master surface onto which a node $S$ of the slave surface is projected. The projection of $S$ onto $P$ can be characterized by two isoparametric coordinate values $\eta_{1 S}$ and $\eta_{2 S}$. As a result of constraining $S$ to $P$, the spatial coordinates of $S$ are expressed as

$$
x_{i S}=x_{i K} a_{K S}
$$

where $K$ ranges over all the nodes defining $P$. The coefficient $a_{K S}$ in Eq. (19) can be expressed in terms of $\eta_{1 S}$ and $\eta_{2 S}$ by the equation

$$
a_{K S}=\phi_{K}^{P}\left(\eta_{1 S}, \eta_{2 S}\right)
$$

where $\phi_{K}^{P}$ is the shape function of node $K$ on face $P$.

The basic idea of the following development is to replace $F_{1}$ with a new boundary which prevents the possibility for overlaps or gaps between the two meshes. The new boundary is composed of two parts. The first part is denoted by $F_{m}$ and consists of the projection of $F_{1}$ onto the master surface (see Figure 1). The second part is denoted by $F_{r}$ and consists of ruled surfaces between the edges of $F_{1}$ and their projections onto the master surface. These two parts of the new boundary are discussed in greater detail subsequently.

Using the divergence theorem, element volume can be expressed in terms of surface integrals over the faces of the element as

$$
V=\sum_{k=1}^{N_{f}} \int_{F_{k}} x_{j} n_{j}^{k} d S \quad \text { for } \quad j=1,2,3
$$

where $N_{f}$ is the number of element faces, $F_{k}$ denotes face $k$, and $\mathbf{n}^{k}=n_{j}^{k} \mathbf{e}_{j}$ is the unit outward normal to $F_{k}$. Let $\hat{V}$ denote the volume of a uniform strain element obtained by replacing $F_{1}$ with the new boundary. It follows from Eq. (21) that

$$
\hat{V}=V-\int_{F_{1}} x_{j} n_{j}^{1} d S-\int_{F_{m}} x_{j} n_{j}^{m} d S+\int_{F_{r}} x_{j} n_{j}^{r} d S \text { for } j=1,2,3
$$

where $\mathbf{n}^{m}=n_{j}^{m} \mathrm{e}_{j}$ is the unit outward normal to $F_{m}$ and $\mathbf{n}^{r}=n_{j}^{r} \mathrm{e}_{j}$ is the unit outward normal to $F_{r}$. Notice that a negative sign is assigned to the third term on the right hand side of Eq. (22) because $\mathbf{n}^{m}$ points into the slave element. The analog to Eq. (6) for the uniform strain element is given by

$$
\hat{B}_{j \hat{I}}=\frac{\partial \hat{V}}{\partial x_{j \hat{I}}}
$$


The index $\hat{I}$ is used instead of $I$ in Eq. (23) to remind the reader that $\hat{V}$ depends on the coordinates of the original element nodes as well as the nodes defining $F_{m}$. To be specific, the index $\hat{I}$ takes on all values of $I$ for the original element except the numbers of nodes constrained to the master boundary. In addition, $\hat{I}$ takes on the numbers of all nodes defining $F_{m}$.

Substituting Eqs. (19) and (22) into Eq. (23), one obtains

$$
\begin{aligned}
\hat{B}_{j \hat{I}}= & B_{j \hat{I}}+a_{\hat{I} S}\left[B_{j S}+\frac{\partial}{\partial x_{j S}}\left(\int_{F_{\tau}} x_{j} n_{j}^{r} d S-\int_{F_{1}} x_{j} n_{j}^{1} d S\right)\right] \\
& +\frac{\partial}{\partial x_{j \hat{I}}}\left(\int_{F_{\tau}} x_{j} n_{j}^{\tau} d S-\int_{F_{m}} x_{j} n_{j}^{m} d S\right) \text { for } j=1,2,3
\end{aligned}
$$

where the index $S$ takes on the numbers of nodes constrained to the master boundary. Notice that $B_{j \hat{I}}=0$ if $\hat{I}$ refers to a node on the master boundary. In addition, $a_{\hat{I} S}$ is zero if $\hat{I}$ refers to node numbers of the original element. The terms involving surface integrals on the right hand side Eq. (24) can be calculated using numerical integration as described in the following paragraphs.

The coordinates of points on $F_{1}$ can be expressed as

$$
x_{i}=x_{i S} \phi_{S}\left(\eta_{1}, \eta_{2}\right)
$$

where $\phi_{S}$ is the shape function of node $S$ on $F_{1}$. Using Eq. (25) and a fundamental result for surface integrals, one obtains

$$
\frac{\partial}{\partial x_{j S}} \int_{F_{1}} x_{j} n_{j}^{1} d S=\int_{A_{\eta 1}} \phi_{S} \epsilon_{j k m} x_{k, 1} x_{m, 2} d A \text { for } j=1,2,3
$$

where $\epsilon_{j k m}$ is the permutation symbol and $A_{\eta 1}$ is the area of integration for $F_{1}$ in the $\eta_{1}-\eta_{2}$ coordinate system. Exact values of the integral on the right hand side of Eq. (26) can be obtained using 2-point Gauss quadrature in two dimensions (4 quadrature points total) for the 8-node hexahedron. For the 20-node and 27-node hexahedron, 3-point Gauss quadrature in two dimensions (9 quadrature points total) is required. Exact values for the 4-node tetrahedron can be obtained using a 1-point quadrature rule for triangular domains while the 10-node tetrahedron requires a 7-point quadrature rule. Quadrature rules for integration over triangular domains are available in Refs. 5 and 9.

The projection of $F_{1}$ onto an element face of the master surface is shown in Figure 2. For each such master element face, the boundary of the projection is defined by a closed polygon consisting of straight-line segments in the isoparametric coordinate system of the master element face. This polygon is decomposed into triangular regions (again in the isoparametric coordinate system of the master element face) as shown to facilitate the calculation of surface integrals.

The coordinates of points on the element face can be expressed as

$$
x_{i}=x_{i M} \phi_{M}\left(\eta_{1}, \eta_{2}\right)
$$


where $\phi_{M}$ is the shape function for node $M$ on the element face. From Eq. (27) one obtains

$$
\frac{\partial}{\partial x_{j M}} \int_{F_{1 f}} x_{j} n_{j}^{1} d S=\int_{A_{\eta f}} \phi_{M} \epsilon_{j k m} x_{k, 1} x_{m, 2} d A \text { for } j=1,2,3
$$

where $F_{1 f}$ denotes the projection of $F_{1}$ onto the element face and $A_{\eta f}$ is the area of integration of the element face in the $\eta_{1}-\eta_{2}$ coordinate system. The integral on the right hand side of Eq. (28) is determined by adding the contributions from each triangular region. The surface integrals can be calculated exactly for each triangular region by using the following quadrature rules for triangular domains: 1-point for 4-node tetrahedron, 4-point for 8-node hexahedron, 7-point for 10-node tetrahedron, 13-point for 20-node hexahedron, and 19-point for 27-node hexahedron. Surface integrals in Eq. (24) over the domain $F_{m}$ are obtained from Eq. (28) by summing the contributions from all involved element faces on the master surface.

Recall that the second part of the boundary to replace $F_{1}$ consists of ruled surfaces between the edges of $F_{1}$ and their projections onto the master surface. These surfaces must be considered only if the edges of $F_{1}$ do not lie entirely on the master surface. By including these surfaces, the "new boundary" of the slave element is ensured to be closed.

An edge of $F_{1}$ and its projection onto the master surface is shown in Figure 3. The spatial coordinates of points along the edge can be expressed as

$$
x_{i e}=x_{i S} \phi_{S e}\left(\xi_{2}\right)
$$

where $\phi_{S e}$ is the shape function of node $S$ on the edge of interest.

The projection of the edge onto a participating element face of the master surface appears as one or more connected straight-line segments in the coordinate system of the element face. For each such segment, the isoparametric coordinates of points along the segment can be expressed as

$$
\begin{aligned}
& \eta_{1}=a_{1}+b_{1} \xi_{2} \\
& \eta_{2}=a_{2}+b_{2} \xi_{2}
\end{aligned}
$$

where the coefficients $a$ and $b$ appearing in Eqs. (30-31) are determined from the projections of nodes and edges of $F_{1}$ described previously. Thus, the spatial coordinates of points along the segment can be expressed as

$$
x_{i g}=x_{i M} \phi_{M}\left(a_{1}+b_{1} \xi_{2}, a_{2}+b_{2} \xi_{2}\right)
$$

where $\phi_{M}$ is the shape function of node $M$ on the element face.

The ruled surface between the segment and the edge is denoted by $F_{g e}$. Spatial coordinates of points on this surface are given by

$$
x_{i}=\left(1-\xi_{1}\right) x_{i g}+\xi_{1} x_{i e}
$$


where $0 \leq \xi_{1} \leq 1$. The bounding values of $\xi_{2}$ which define $F_{g e}$ are determined from the projections described previously. It follows from Eqs. (29-33) that

$$
\begin{aligned}
& \frac{\partial}{\partial x_{j M}} \int_{F_{g e}} x_{j} n_{j}^{r} d S=\int_{A_{\xi r}} \phi_{M} \epsilon_{j k m} \tilde{x}_{k, 1} \tilde{x}_{m, 2}\left(1-\xi_{1}\right) d A \text { for } j=1,2,3 \\
& \frac{\partial}{\partial x_{j S}} \int_{F_{g e}} x_{j} n_{j}^{r} d S=\int_{A_{\xi r}} \phi_{S e} \epsilon_{j k m} \tilde{x}_{k, 1} \tilde{x}_{m, 2} \xi_{1} d A \text { for } j=1,2,3
\end{aligned}
$$

where $A_{\xi r}$ is the area of integration for $F_{g e}$ in the $\xi_{1}-\xi_{2}$ coordinate system, and

$$
\begin{aligned}
& \tilde{x}_{i, 1}=x_{i S} \phi_{S e}-x_{i M} \phi_{M}\left(a_{1}+b_{1} \xi_{2}, a_{2}+b_{2} \xi_{2}\right) \\
& \tilde{x}_{i, 2}=x_{i M}\left[\left(\partial \phi_{M} / \partial \eta_{1}\right) b_{1}+\left(\partial \phi_{M} / \partial \eta_{2}\right) b_{2}\right]\left(1-\xi_{1}\right)+x_{i S}\left(\partial \phi_{S e} / \partial \xi_{2}\right) \xi_{1}
\end{aligned}
$$

The integrals on the right hand sides of Eqs. (34-35) can be calculated exactly using a 2-point Gauss quadrature rule in the $\xi_{1}$ direction. For edges on the slave surface with three or fewer nodes, the following quadrature rules for the $\xi_{2}$ direction are sufficient: 3-point for a 4-node tetrahedron or 8-node hexahedron with a face on the master surface, 4-point for a 6-node tetrahedron or a 20-node hexahedron, and 6-point for a 27-node hexahedron. The surface integrals in Eq. (24) over the domain $F_{\tau}$ are obtained from Eqs. (34-35) by summing the contributions from all involved segments on the master surface.

If the slave surface consists entirely of uniform strain elements, then all the necessary corrections are contained in $\hat{B}_{j \hat{I}}$. By using Eqs. (24) to calculate $\hat{B}_{j \hat{I}}$ for elements with faces on the slave surface, one can perform analyses of connected meshes for both linear and nonlinear problems. A general method of hourglass control [10] can also be used to stabilize any elements on the boundary with spurious zero energy deformation modes.

The remainder of this section is concerned with extending the method to accommodate more commonly used finite elements on the slave surface. Although we believe the method can be extended easily to nonlinear problems, attention is restricted presently to the linear case. Needless to say, many problems of practical interest are in this category.

Prior to any modifications, the stiffness matrix $K$ of an element with a face on the slave surface can be expressed as

$$
K=K_{u}+K_{r}
$$

where $K_{u}$ denotes the uniform strain portion of $K$ and $K_{r}$ is the remainder. The matrix $K_{u}$ is defined as

$$
K_{u}=V C^{T} D C
$$

where $D$ is a material matrix that is assumed constant throughout the element. Recall that $V$ is the element volume and $C$ is given by Eq. (16). Substituting Eq. (39) into Eq. (38) and solving for $K_{r}$ yields

$$
K_{r}=K-V C^{T} D C
$$

Let $u^{l}$ denote the vector $u$ (see Eq. 17) obtained by sampling a linear displacement field at the nodes. The nodal forces $f^{l}$ associated with $u^{l}$ are given by

$$
f^{l}=K u^{l}
$$


For a properly formulated element, one has

$$
K_{u} u^{l}=f^{l}
$$

and

$$
K_{r} u^{l}=0
$$

If Eq. (42) does not hold, then $K_{u} u^{l} \neq f^{l}$ and elements based on the uniform strain approach would fail a first-order patch test. Equation (43) implies that $K_{r}$ does not contribute to the nodal forces for linear displacement fields.

The basic idea of the following development is to alter the uniform strain portion of the stiffness matrix while leaving $K_{r}$ unchanged. Let $\hat{u}$ denote the displacement vector for nodes associated with the index $\hat{I}$ (see discussion following Eq. 23). Based on the constraints in Eq. (19), one may express $u$ in terms of $\hat{u}$ as

$$
u=G \hat{u}
$$

where $G$ is a transformation matrix. The modified stiffness matrix $\hat{K}$ of the element is defined as

$$
\hat{K}=\hat{V} \hat{C}^{T} D \hat{C}+G^{T} K_{r} G
$$

where $\hat{C}$ denotes the matrix $C$ (see Eq. 16) associated with $\hat{B}_{j \hat{I}}$ (see Eq. 24). The stiffness . matrix $K_{m s}$ obtained using the standard master-slave approach is given by

$$
K_{m s}=G^{T} K G
$$

Comparing $\hat{K}$ with $K_{m s}$, one finds that

$$
\hat{K}-K_{m s}=\hat{V} \hat{C}^{T} D \hat{C}-G^{T}\left(V C^{T} D C\right) G
$$

The right hand side of Eq. (47) is simply the difference between the uniform strain portions of $\hat{K}$ and $K_{m s}$. If continuity at the master-slave interface holds by satisfying Eq. (44) alone, then the surfaces integrals in Eq. (24) sum to zero and $\hat{K}=K_{m s}$. Thus, under such conditions, the present method and the standard master-slave approach are equivalent.

Prior to element modifications, the strain $\epsilon$ in an element on the slave surface can be expressed as

$$
\epsilon=\mathrm{Cu}+\mathrm{Hu}
$$

where $C u$ is the uniform strain (see Eq. 14) and $H u$ is the remainder. The modified element strain $\hat{\epsilon}$ is defined as

$$
\hat{\epsilon}=\hat{C} \hat{u}+H u
$$

Equation (49) is used to calculate the strains in elements with faces on the slave surface.

One might erroneously consider developing a modified stiffness matrix $\hat{K}_{\hat{\epsilon}}$ based on Eq. (49). The result is

$$
\hat{K}_{\hat{\epsilon}}=\hat{V} \hat{C}^{T} D \hat{C}+\int_{\hat{\Omega}}\left[\hat{C}^{T} D H G+G^{T} H^{T} D \hat{C}+G^{T} H^{T} D H G\right] d V
$$


where $\hat{\Omega}$ denotes the domain of the element with face $F_{1}$ replaced by the new boundary. The difficulties with using $\hat{K}_{\hat{\varepsilon}}$ for an element formulation are twofold. First, it may not be simple to evaluate the integral in Eq. (50) because the domain $\hat{\Omega}$ could be irregular. Second, and more importantly, such an element formulation does not pass the patch test. To explain this fact, let $\hat{u}^{l}$ denote the vector $\hat{u}$ obtained by sampling a linear displacement field. In general, one has $\hat{K} \hat{u}^{l} \neq \hat{K}_{\hat{\varepsilon}} \hat{u}^{l}$ since the product $\hat{C} \hat{u}^{l}$ is not necessarily zero.

In summary; the present method alters the formulations of elements on the slave surface by accounting correctly for the volume between the two meshes that is present either initially or during deformation. A method that does not require changes to element formulations for elements on the master or slave surfaces may be preferable in certain instances. We are currently investigating such a method based on constraint equations and the volume accounting principles explored in this study.

\section{Example Problems}

All the example problems in this section assume small deformations of a linear, elastic, isotropic material with Young's modulus $E=10^{7}$ and Poisson's ratio $\nu=0.3$. In this case, the material matrix $D$ can be expressed as

$$
D=\left[\begin{array}{cccccc}
2 G+\lambda & \lambda & \lambda & 0 & 0 & 0 \\
\lambda & 2 G+\lambda & \lambda & 0 & 0 & 0 \\
\lambda & \lambda & 2 G+\lambda & 0 & 0 & 0 \\
0 & 0 & 0 & G & 0 & 0 \\
0 & 0 & 0 & 0 & G & 0 \\
0 & 0 & 0 & 0 & 0 & G
\end{array}\right]
$$

where

$$
G=\frac{E}{2(1+\nu)}
$$

and

$$
\lambda=\frac{E \nu}{(1+\nu)(1-2 \nu)}
$$

Five different element types are considered in the example problems. These include the 4-node tetrahedron (T4), eight-node hexahedron $(H 8)$, ten-node tetrahedron $(T 10), 20$ node hexahedron ( $H 20)$, and 27-node hexahedron ( $H 27)$. Stiffness matrices of the various elements are calculated using numerical integration. The following quadrature rules in three dimensions are used for the hexahedral elements: 2-point for 8-node hexahedron, 3-point for 20-node hexahedron, and 3-point for 27-node hexahedron. Single-point and 5-point quadrature rules for tetrahedral domains are used for element types $T 4$ and $T 10$, respectively.

Two meshes connected at a shared boundary are used in all the example problems. Mesh 1 is initially bounded by the the six sides $x_{1}=0, x_{1}=h_{1}, x_{2}=0, x_{2}=h_{2}, x_{3}=0$ and $x_{3}=h_{3}$ while Mesh 2 is initially bounded by $x_{1}=h_{1}, x_{1}=2 h_{1}, x_{2}=0, x_{2}=h_{2}, x_{3}=0$ and $x_{3}=h_{3}$ (see Figure 4). Each mesh consists of one of the element types described in the previous paragraph. The number of element edges in direction $i$ for mesh $m$ is designated 
by $n_{i m}$. Thus, all the meshes in Figure 4 have $n_{11}=n_{21}=n_{31}=2$ and $n_{12}=n_{22}=n_{32}=3$. Specific mesh configurations are designated by the element type for Mesh 1 followed by the element type for Mesh 2.

Calculated values of the energy norm of the error are presented for purposes of comparison and for the investigation of convergence rates. The energy norm of the error is a measure of the accuracy of a finite element approximation and is defined as

$$
e=\left[\sum_{k \in \mathcal{I}} \int_{\Omega_{k}}\left(\epsilon^{f e}-\epsilon^{e x a c t}\right)^{T} D\left(\epsilon^{f e}-\epsilon^{e x a c t}\right) d V\right]^{1 / 2}
$$

where $\Omega_{k}$ is the domain of element $k$ and $\epsilon^{f e}$ and $\epsilon^{\text {exact }}$ denote the finite element and exact strains, respectively. The symbol $\mathcal{I}$ denotes the set of all element numbers for the two meshes. Calculation of energy norms for hexahedral and tetrahedral elements is based on the quadrature rules for element types $H 20$ and $T 10$, respectively.

\section{Example 3.1}

The first example is concerned with a uniaxial tension patch test and highlights some of the differences between the standard master-slave approach and the present method. The boundary conditions for the problem are given by

$$
\begin{array}{r}
u_{1}\left(0, x_{2}, x_{3}\right)=0 \\
u_{2}(0,0,0)=0 \\
u_{3}(0,0,0)=0 \\
u_{3}\left(0, h_{2}, 0\right)=0
\end{array}
$$

and

$$
\sigma_{11}\left(2 h_{1}, x_{2}, x_{3}\right)=1
$$

The exact solution for the displacement is given by

$$
\begin{aligned}
& u_{1}\left(x_{1}, x_{2}, x_{3}\right)=x_{1} / E \\
& u_{2}\left(x_{1}, x_{2}, x_{3}\right)=-\nu x_{2} / E \\
& u_{3}\left(x_{1}, x_{2}, x_{3}\right)=-\nu x_{3} / E
\end{aligned}
$$

The exact solution for stresses has all components equal to zero except for $\sigma_{11}$ which equals unity. All the meshes used in the example have $h_{1}=5, h_{2}=10, h_{3}=10, n_{11}=n_{21}=n_{31}=n$ and $n_{12}=n_{22}=n_{32}=3 n / 2$ where $n$ is a positive even integer.

Several analyses with $n=2$ were performed to evaluate the method. Using all five element types for Mesh 1 and Mesh 2 resulted in 25 different mesh configurations. Nodes internal to the meshes and along the master-slave interface were moved randomly so that all the elements were initially distorted. Following the initial movement of nodes, nodes on the slave boundary were repositioned to lie on the master boundary. It is noted that gaps and overlaps still remained between the two meshes after repositioning the slave surface nodes 
(see Figure 5). The two meshes were alternately designated as master and slave. In all cases the patch test was passed. That is, the calculated element stresses and nodal displacements were in agreement with the exact solution to machine precision.

The remaining discussion for this example deals with results obtained using the standard master-slave approach with Mesh 1 designated as master. The minimum and maximum values of $\sigma_{11}$ at centroids of elements with faces on the slave surface are shown in Table 1 for mesh configurations $H 8 H 8, H 20 H 20, T 4 T 4$ and $T 10 T 10$ for a variety of mesh resolutions. It is clear from the table that refinement of the meshes does not improve the accuracy of the solution at the shared boundary. In addition, the errors in stress at the interface are greater for mesh configuration $H 20 H 20$ than for $H 8 H 8$. Figure 6 shows the values of $\sigma_{11}$ for mesh configuration $H 8 H 8$ with $n=4$. The same information is shown in Figure 7 for mesh configuration $\mathrm{H} 20 \mathrm{H} 20$.

Plots of the energy norm of the error for mesh configurations $H 8 H 8$ and $H 20 H 20$ are shown in Figure 8. It is clear that the energy norms decrease with mesh refinement, but the convergence rates are significantly lower than those expected for elements in a single unconnected mesh. The slopes of lines connecting the first two data points are approximately 0.51 and 0.50 for $\mathrm{H} 8 \mathrm{H} 8$ and $\mathrm{H} 2 \mathrm{OH} 2 \mathrm{O}$, respectively. In contrast, the energy norms of the error for a single mesh of $H 8$ or undistorted $H 20$ elements have slopes which asymptotically approach 1 and 2, respectively, in the absence of singularities. The fact that displacement continuity is not satisfied at the shared boundary severely degrades the convergence characteristics of the connected meshes.

We note that the results presented in Table 1 and Figures 6-8 are for the "best case" scenario of connecting two regular meshes that conform initially. In general, two dissimilar meshes will not conform initially at all locations if the shared boundary is curved. Use of the standard master-slave approach in such cases may result in even greater errors.

\section{Example 3.2}

The second example investigates convergence rates for the present method. The specific problem considered is pure bending. The problem description is identical to Example 3.1 with the exception that the boundary condition at $x_{1}=2 h_{1}$ is replaced by

$$
\sigma_{11}\left(2 h_{1}, x_{2}, x_{3}\right)=h_{2} / 2-x_{2}
$$

The exact solution has all of the stress components equal to zero except for $\sigma_{11}$ which is given by

$$
\sigma_{11}\left(x_{1}, x_{2}, x_{3}\right)=h_{2} / 2-x_{2}
$$

In all cases Mesh 1 was designated as master.

Plots of the energy norm of the error are shown in Figure 9 for mesh configurations $\mathrm{H} 8 \mathrm{H} 8$ and $H 20 H 20$. The slopes of lines connecting the first two data points are approximately 1.00 and 1.76 for $H 8 H 8$ and $H 20 H 20$, respectively. Notice that a convergence rate of unity is achieved by mesh configuration $H 8 H 8$. Although the slopes of line segments are greater for mesh configuration $H 20 H 20$, the optimal slope of 2 is not achieved. One should not expect 
to obtain a convergence rate of 2 with the present method since corrections are made only to satisfy first-order patch tests. Nevertheless, the results for mesh configuration H2OH2O are more accurate than those for $\mathrm{H} 8 \mathrm{H} 8$. Although the asymptotic rate of convergence for $\mathrm{H} 2 \mathrm{OH} 2 \mathrm{O}$ is not clear from the figure, it is bounded below by unity.

\section{Example 3.3}

The final example demonstrates the freedom to designate master and slave boundaries independently of the resolutions of the two meshes. We consider again a problem of pure bending for mesh configuration $H 8 H 8$ with Mesh 1 designated as master. The boundary conditions are given by

$$
\begin{array}{r}
u_{2}\left(x_{1}, 0, x_{3}\right)=0 \\
u_{3}(0,0,0)=0 \\
u_{1}(0,0,0)=0 \\
u_{1}\left(0,0, h_{3}\right)=0
\end{array}
$$

and

$$
\sigma_{22}\left(x_{1}, h_{2}, x_{3}\right)=h_{1}-x_{1}
$$

The exact solution has all of the stress components equal to zero except for $\sigma_{22}$ which is given by

$$
\sigma_{22}\left(x_{1}, x_{2}, x_{3}\right)=h_{1}-x_{1}
$$

All the meshes used in the example have $h_{1}=1, h_{2}=10, h_{3}=1, n_{11}=n_{12}=n$ and $n_{31}=n_{32}=n$. Two different cases are considered for the mesh resolutions in the 2-direction. For Case $1, n_{21}=5 n$ and $n_{22}=10 n$. For Case 2, $n_{21}=10 n$ and $n_{22}=5 n$. Thus, for Case 1 the mesh resolution in the 2-direction of the slave surface is twice that of the master surface. In contrast, the mesh resolution in the 2-direction of the master surface is twice that of the slave surface for Case 2. Mesh resolutions in the 1 and 3 directions for Meshes 1 and 2 are the same for both cases. Results for Case 1 are identical to those obtained using the standard master-slave approach since the meshes are conforming in this case.

Plots of the energy norm of the error are shown in Figure 10 for Case 1 and Case 2. Notice that Case 2 is consistently more accurate for all the mesh resolutions considered. In order to investigate the cause of these differences, the shear stress component $\sigma_{12}$ was calculated at the centroids of elements with faces on the slave surface. Results of these calculations are presented in Figures 11 and 12 for $n=2$. The exact value of $\sigma_{12}$ for this example is zero over the entire domain of both meshes. Notice that the magnitudes of $\sigma_{12}$ are significantly smaller for Case 2 than Case 1. It is thought that results for Case 2 are more accurate than those for Case 1 because fewer degrees of freedom are constrained at the shared boundary. This example shows that there may be a preferred choice for the master boundary in certain instances.

\section{Conclusions}


A systematic and straightforward method is presented for connecting dissimilar finite element meshes in three dimensions. By modifying the boundaries of elements with faces on the slave surface, corrections can be made to element formulations such that first-order patch tests are passed. The method can be used to connect meshes with different element types. In addition, master and slave surfaces can be designated independently of the resolutions of the two meshes.

A simple uniaxial stress example demonstrated several of the advantages of the present method over the standard master-slave approach. Although the energy norm of the error decreased with mesh refinement for the master-slave approach, the convergence rates were significantly lower than those for elements in a single unconnected mesh. Calculated stresses in elements with faces on the shared boundary had errors up to 13 and 24 percent for connected meshes of 8-node and 20-node hexahedral elements, respectively. For 4-node and 10-node tetrahedral elements, the errors were in excess of 21 percent. Moreover, these errors could not be reduced with mesh refinement.

A convergence rate of unity for the energy norm of the error was achieved for a pure bending example using connected meshes of 8-node hexahedral elements. This convergence rate is consistent with that of a single mesh of 8-node hexahedral elements. More accurate results were obtained for connected meshes of 20 -node hexahedral elements, but|a convergence rate of two was not achieved. The optimal convergence rate of two was not achieved in this case because element corrections are made only to satisfy first-order patch tests.

The final example showed that improved accuracy can be achieved in certain instances by allowing the master surface to have a greater number of nodes than the slave surface. Standard practice commonly requires the master surface to have fewer numbers of nodes. By relaxing this constraint, improved results were obtained as measured by the energy norm of the error and stresses along the shared boundary. 


\section{References}

1. K. K. Ang and S. Valliappan, 'Mesh Grading Technique using Modified Isoparametric Shape Functions and its Application to Wave Propagation Problems,' International Journal for Numerical Methods in Engineering, 23, 331-348, (1986).

2. L. Quiroz and P. Beckers, 'Non-Conforming Mesh Gluing in the Finite Element Method,' International Journal for Numerical Methods in Engineering, 38, 2165-2184 (1995).

3. D. Rixen, C. Farhat and M. Géradin, 'A Two-Step, Two-Field Hybrid Method for the Static and Dynamic Analysis of Substructure Problems with Conforming and Nonconforming Interfaces,' Computer Methods in Applied Mechanics and Engineering, 154, 229-264 (1998).

4. T. Y. Chang, A. F. Saleeb and S. C. Shyu, 'Finite Element Solutions of Two-Dimensional Contact Problems Based on a Consistent Mixed Formulation,' Computers and Structures, 27, 455-466 (1987).

5. O. C. Zienkiewicz and R. L. Taylor, The Finite Element Method, Vol. 1, 4th Ed., McGraw-Hill, New York, New York, 1989.

6. C. R. Dohrmann, S. W. Key and M. W. Heinstein, 'A Method for Connecting Dissimilar Finite Element Meshes in Two Dimensions', submitted to International Journal for Numerical Methods in Engineering.

7. C. R. Dohrmann and S. W. Key; 'A Transition Element for Uniform Strain Hexahedral and Tetrahedral Finite Elements,' to appear in International Journal for Numerical Methods in Engineering.

8. D. P. Flanagan and T. Belytschko, 'A Uniform Strain Hexahedron and Quadrilateral with Orthogonal Hourglass Control', International Journal for Numerical Methods in Engineering, 17, 679-706 (1981).

9. M. E. Laursen and M. Gellert, 'Some Criteria for Numerically Integrated Matrices and Quadrature Formulas for Triangles,' International Journal for Numerical Methods in Engineering, 12, 67-76 (1978).

10. C. R. Dohrmann, S. W. Key, M. W. Heinstein and J. Jung, 'A Least Squares Approach for Uniform Strain Triangular and Tetrahedral Finite Elements', International Journal for Numerical Methods in Engineering, 42, 1181-1197 (1998). 
Table 1: Minimum and maximum values of $\sigma_{11}$ at centroids of elements with faces on the slave surface for Example 3.1. The results presented were obtained using the standard masterslave approach for different resolutions of mesh configurations $H 8 H 8, H 20 H 20, T 4 T 4$ and T10T10. The exact value of $\sigma_{11}$ is unity.

\begin{tabular}{|c|c|c|c|c|c|c|c|c|}
\hline$n$ & \multicolumn{2}{|c|}{$H 8 H 8$} & \multicolumn{2}{c|}{$H 20 H 20$} & \multicolumn{2}{c|}{ T4T4 } & \multicolumn{2}{c|}{ T10T10 } \\
\hline & $\min$ & $\max$ & $\min$ & $\max$ & $\min$ & $\max$ & $\min$ & $\max$ \\
\hline 2 & 0.9406 & 1.1196 & 0.7697 & 1.1009 & 0.7872 & 1.1350 & 0.7898 & 1.1082 \\
4 & 0.9313 & 1.1298 & 0.7644 & 1.1064 & 0.7689 & 1.1649 & 0.7858 & 1.1209 \\
6 & 0.9305 & 1.1294 & 0.7642 & 1.1061 & 0.7651 & 1.1687 & 0.7854 & 1.1208 \\
8 & 0.9304 & 1.1292 & 0.7642 & 1.1061 & 0.7639 & 1.1694 & - & - \\
\hline
\end{tabular}




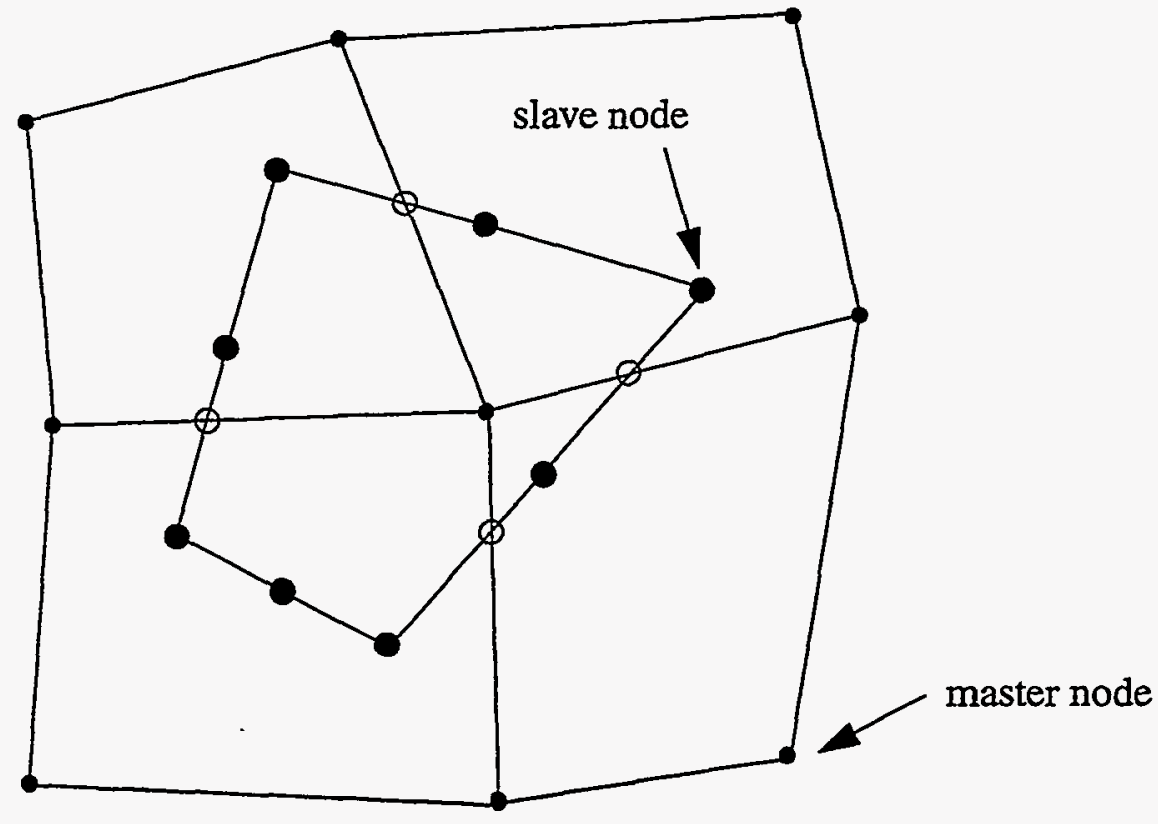

Figure 1: Projection of an element face $F_{1}$ of the slave surface onto the master surface. Larger filled circles designate nodes on the slave surface constrained to the master surface. Smaller filled circles designate nodes on the master surface. Circles that are not filled designate the projections of slave element edges onto master element edges. 


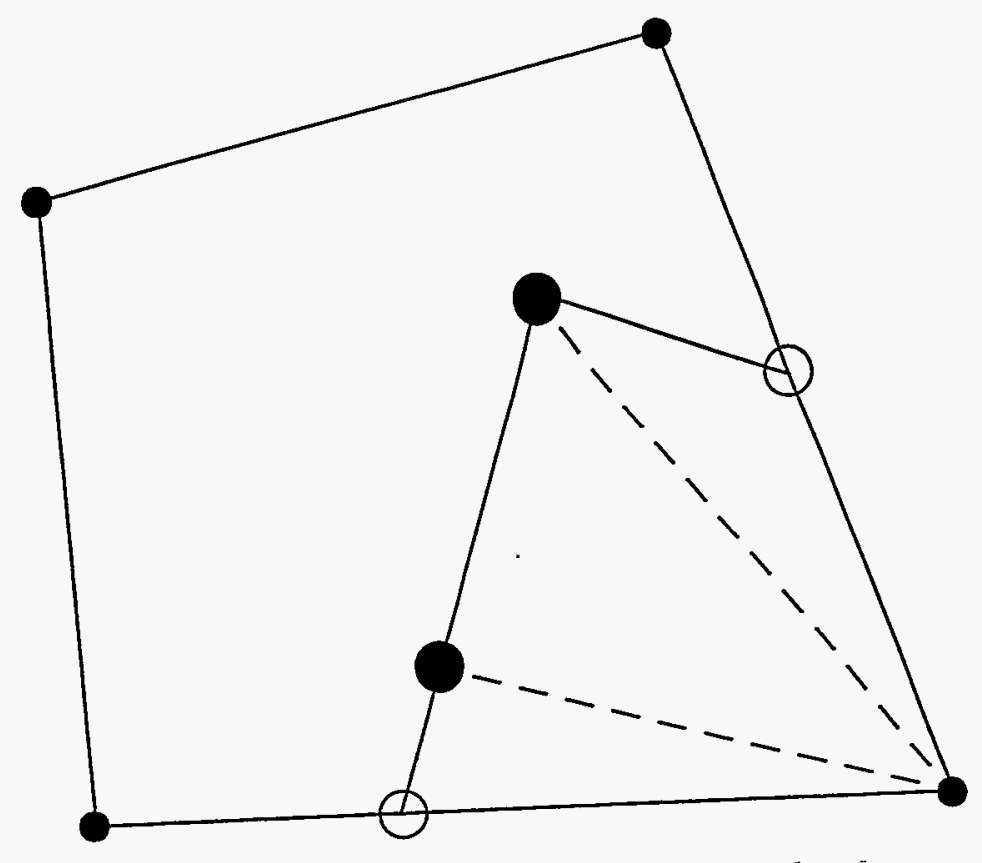

Figure 2: Projection of $F_{1}$ onto an element face of the master surface (see top left corner of Figure 1). In the coordinate system of the element face, the triangular regions have straight edges and lie in a single plane. The domain of the projection of $F_{1}$ onto the element face is divided into triangular regions for the purpose of calculating surface integrals over $F_{m}$. 


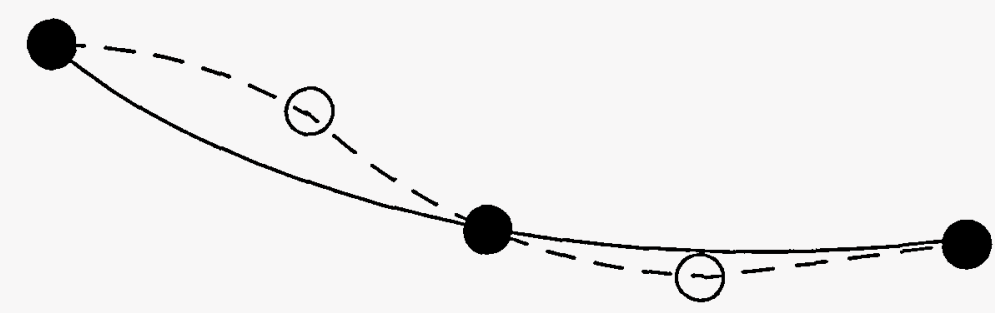

Figure 3: An edge of $F_{1}$ (solid line) and its projection onto the master surface (dashed line) viewed from a direction nearly orthogonal to $F_{1}$. The edge shown spans three different element faces on the master surface. The projection of the edge onto the master surface is a piecewise continuous line with possible discontinuities in slope at edges on the master surface. The solid and dashed lines appear as straight lines in the coordinate systems of element faces on the slave and master surfaces, respectively. 


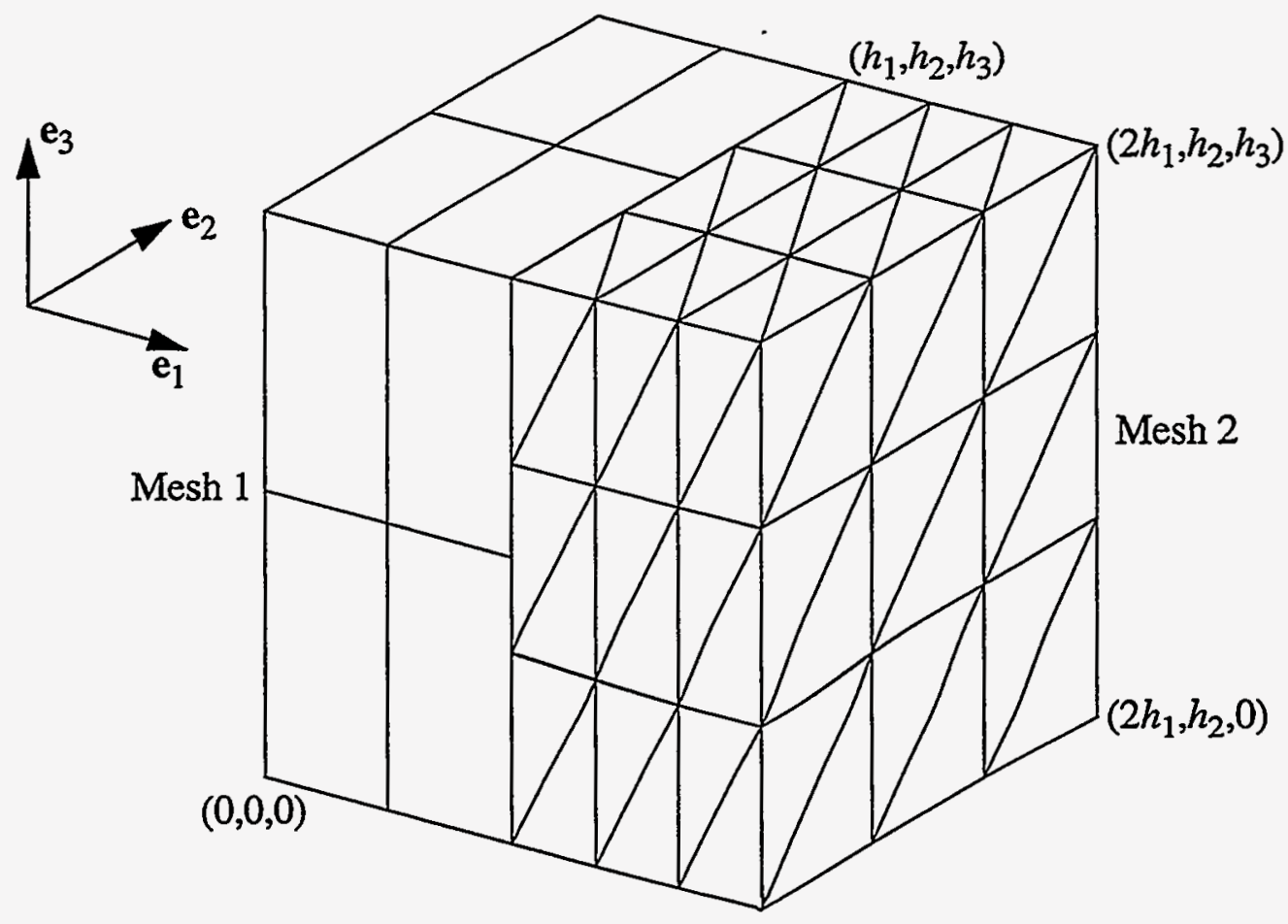

(a)

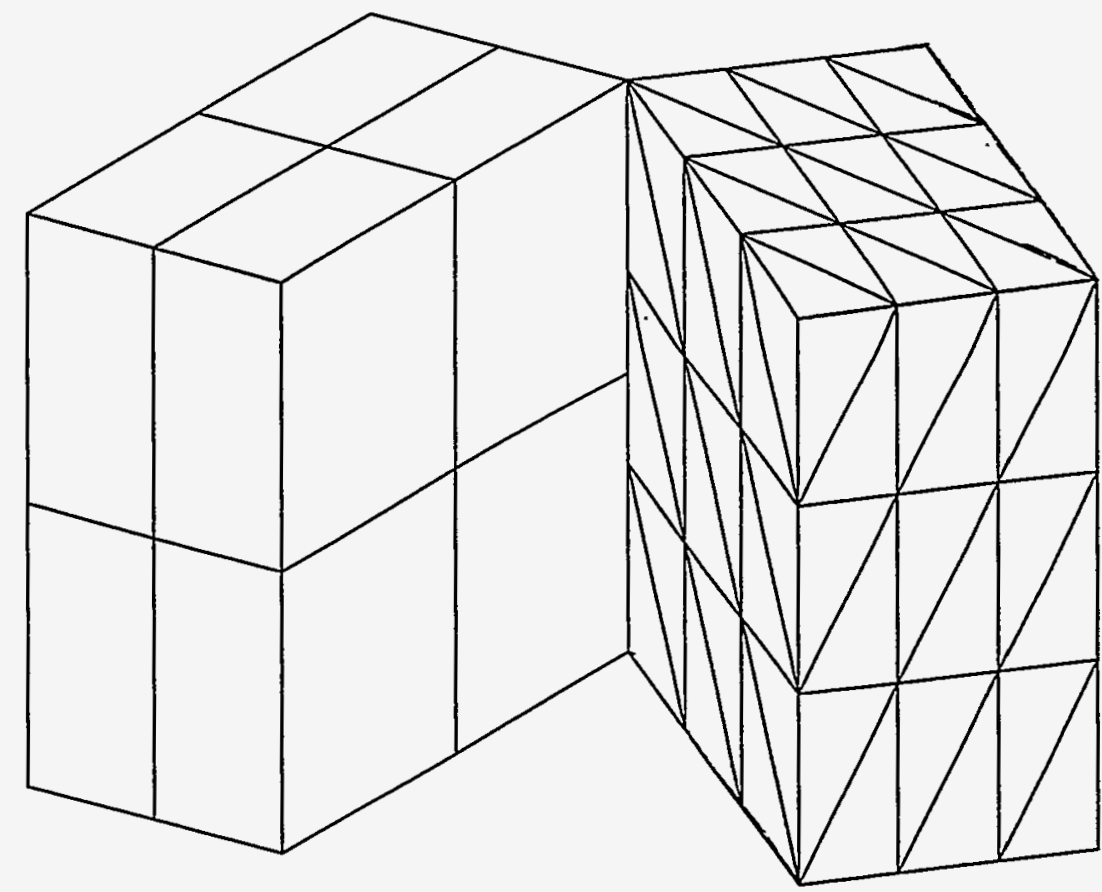

(b)

Figure 4: (a) Mesh configuration H8T4 with $n_{11}=n_{21}=n_{31}=2$ and $n_{12}=n_{22}=n_{32}=3$, (b) opened view of meshes revealing shared boundary. 


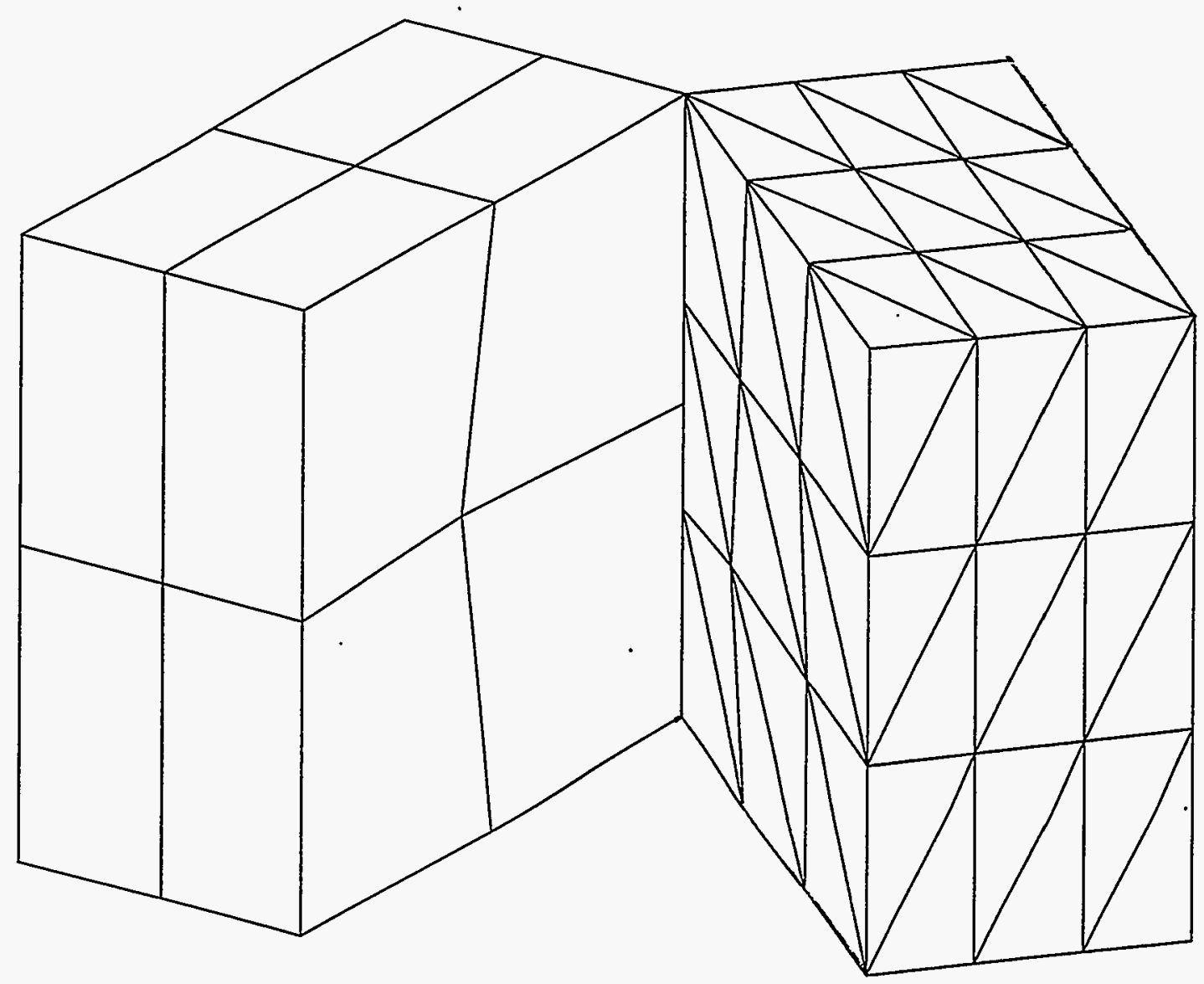

Figure 5: Opened view of mesh configuration H8T4 with distorted elements. Although the slave nodes are repositioned to lie on the master surface, gaps and overlaps still remain between the two meshes because of the distorted element faces. Patch tests for this mesh configuration and others were passed in all cases using the present method. 


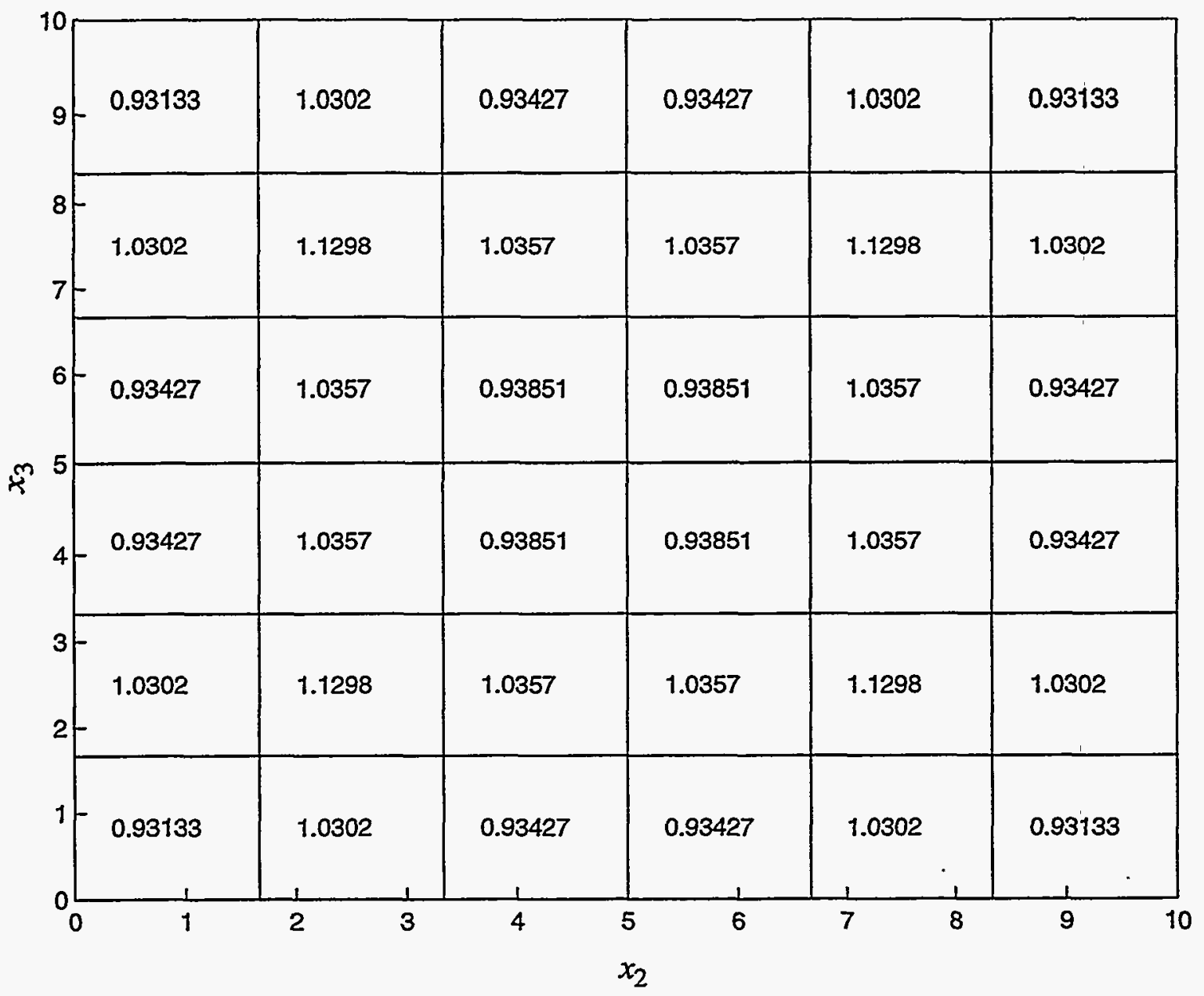

Figure 6: Stress component $\sigma_{11}$ at centroids of elements with faces on the slave surface for Example 3.1. Results presented are for mesh configuration $\mathrm{H} 8 \mathrm{H8}$ using the standard master-slave approach. 


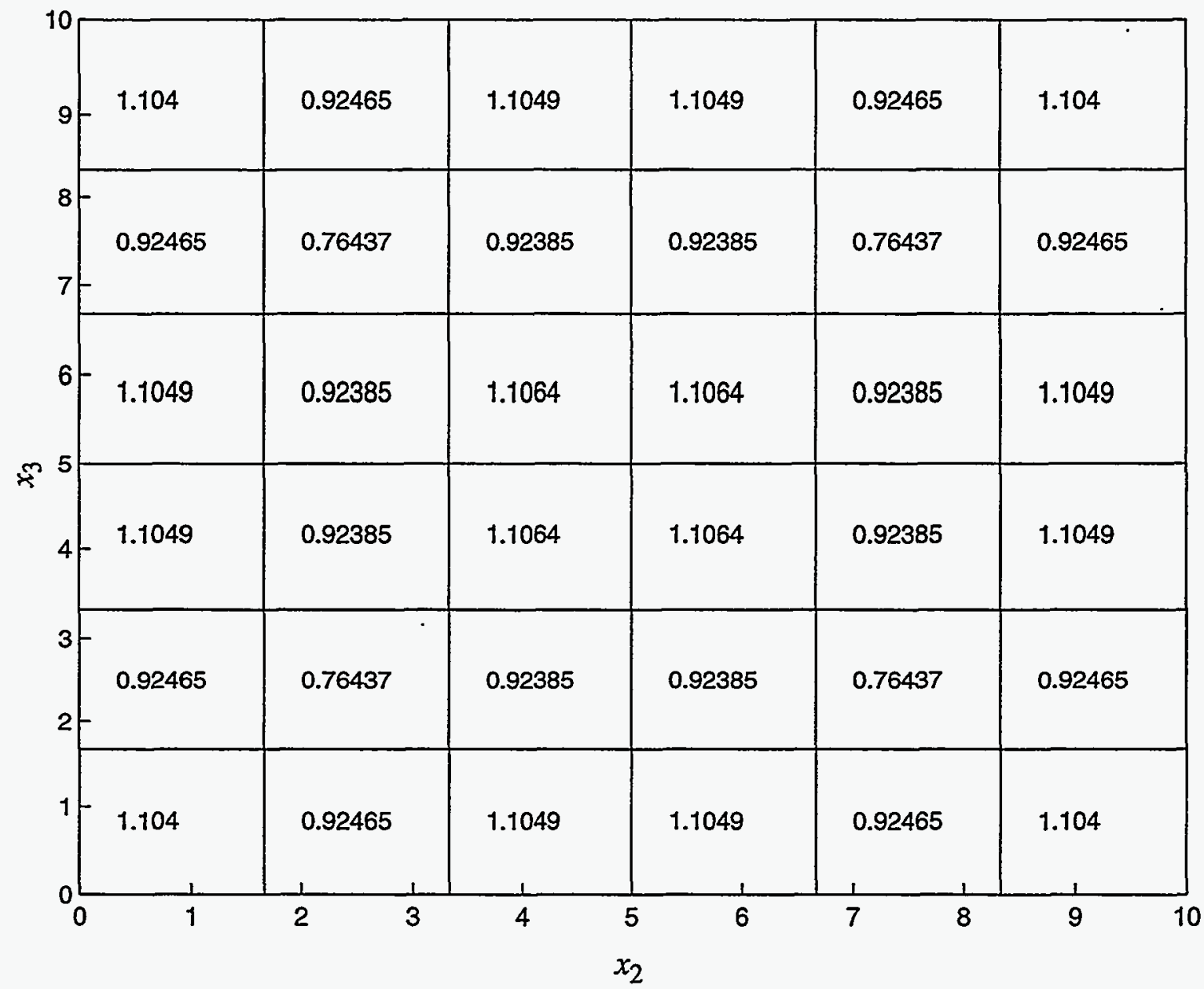

Figure 7: Stress component $\sigma_{11}$ at centroids of elements with faces on the slave surface for Example 3.1. Results presented are for mesh configuration $H 20 H 20$ using the standard master-slave approach. 


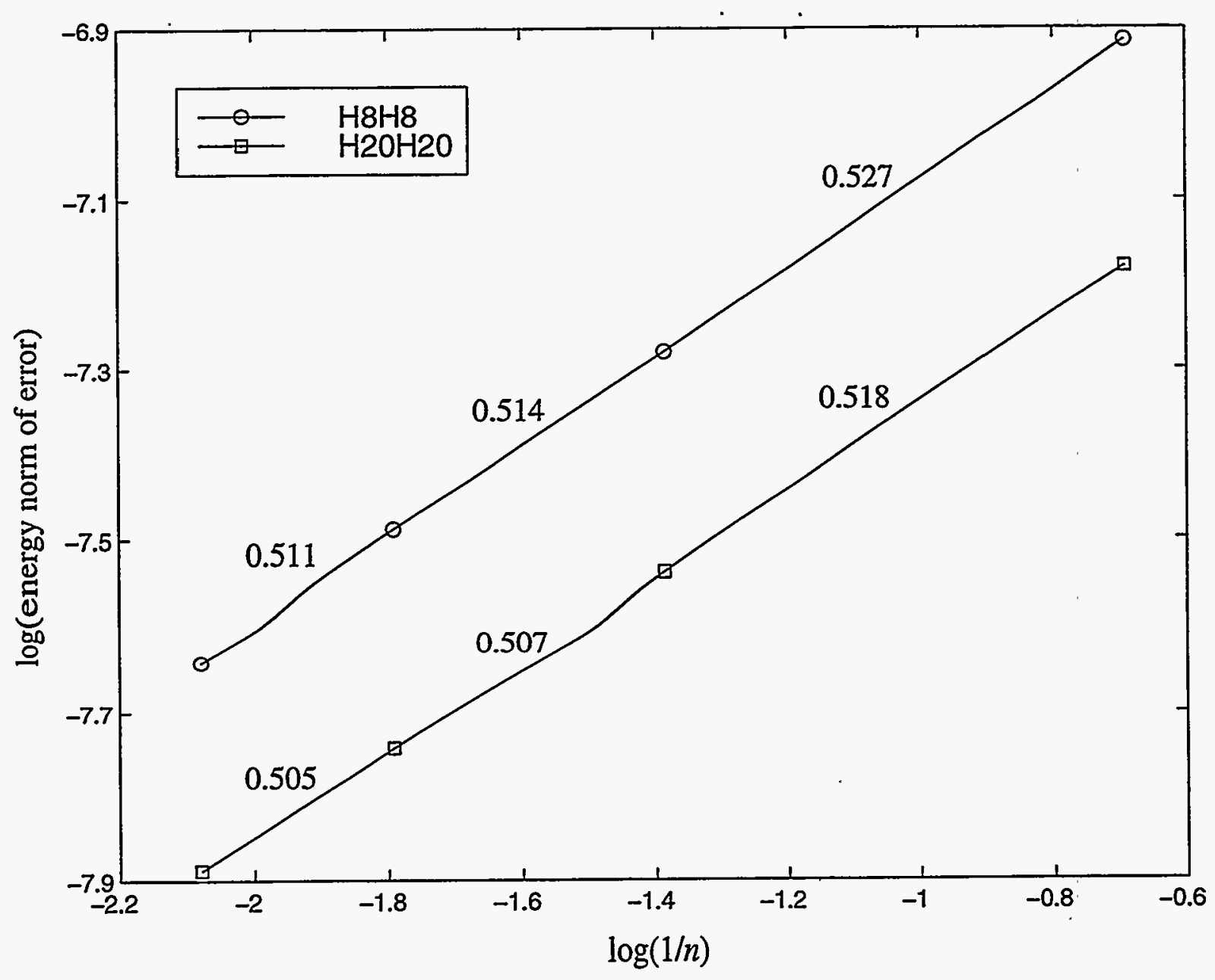

Figure 8: Energy norms of the error for Example 3.1 obtained using the standard master-slave approach. Slopes of lines connecting the data points are shown above the line segments. 


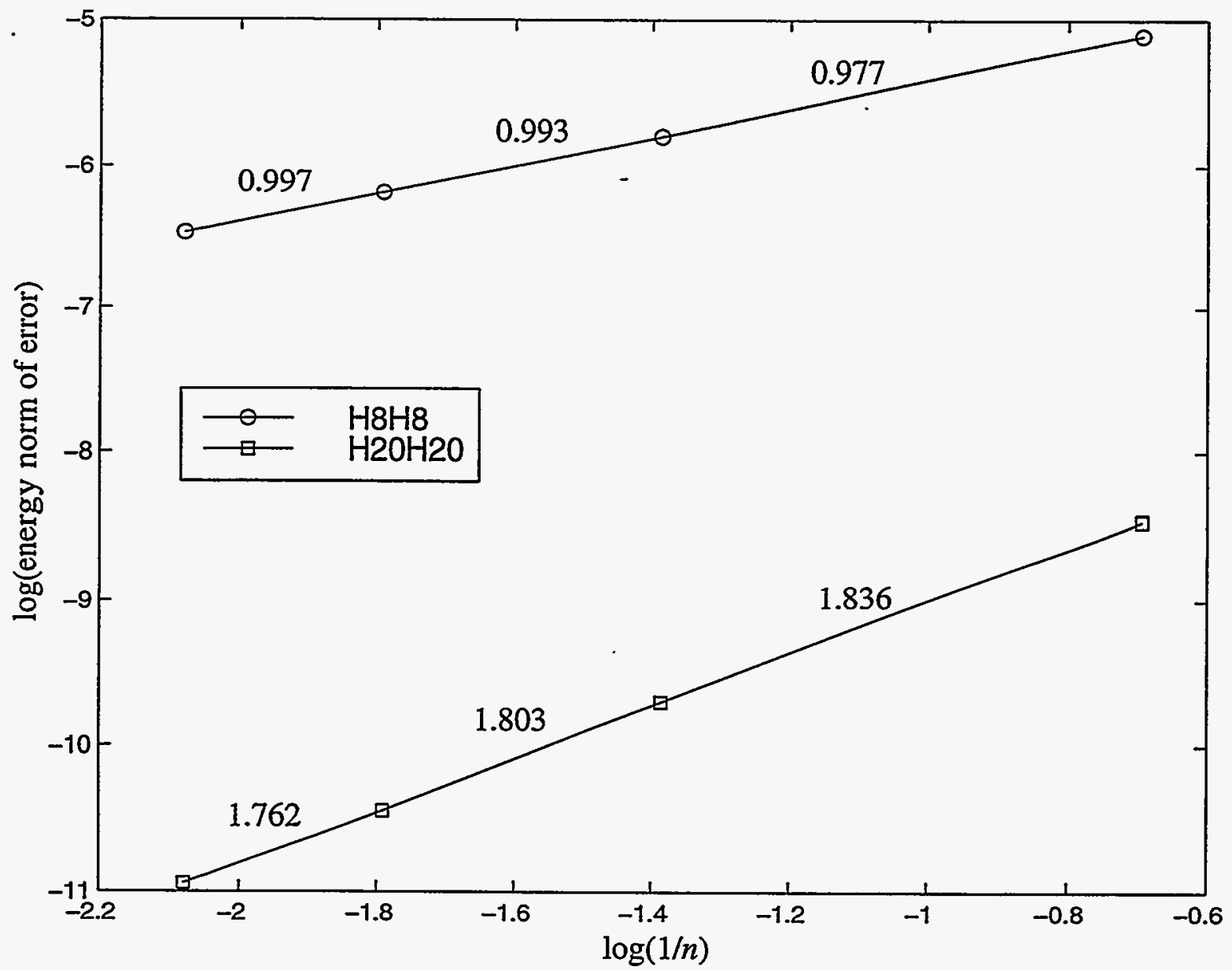

Figure 9: Energy norms of the error for Example 3.2 obtained using the present method. Slopes of lines connecting the data points are shown above the line-segments. 
1.

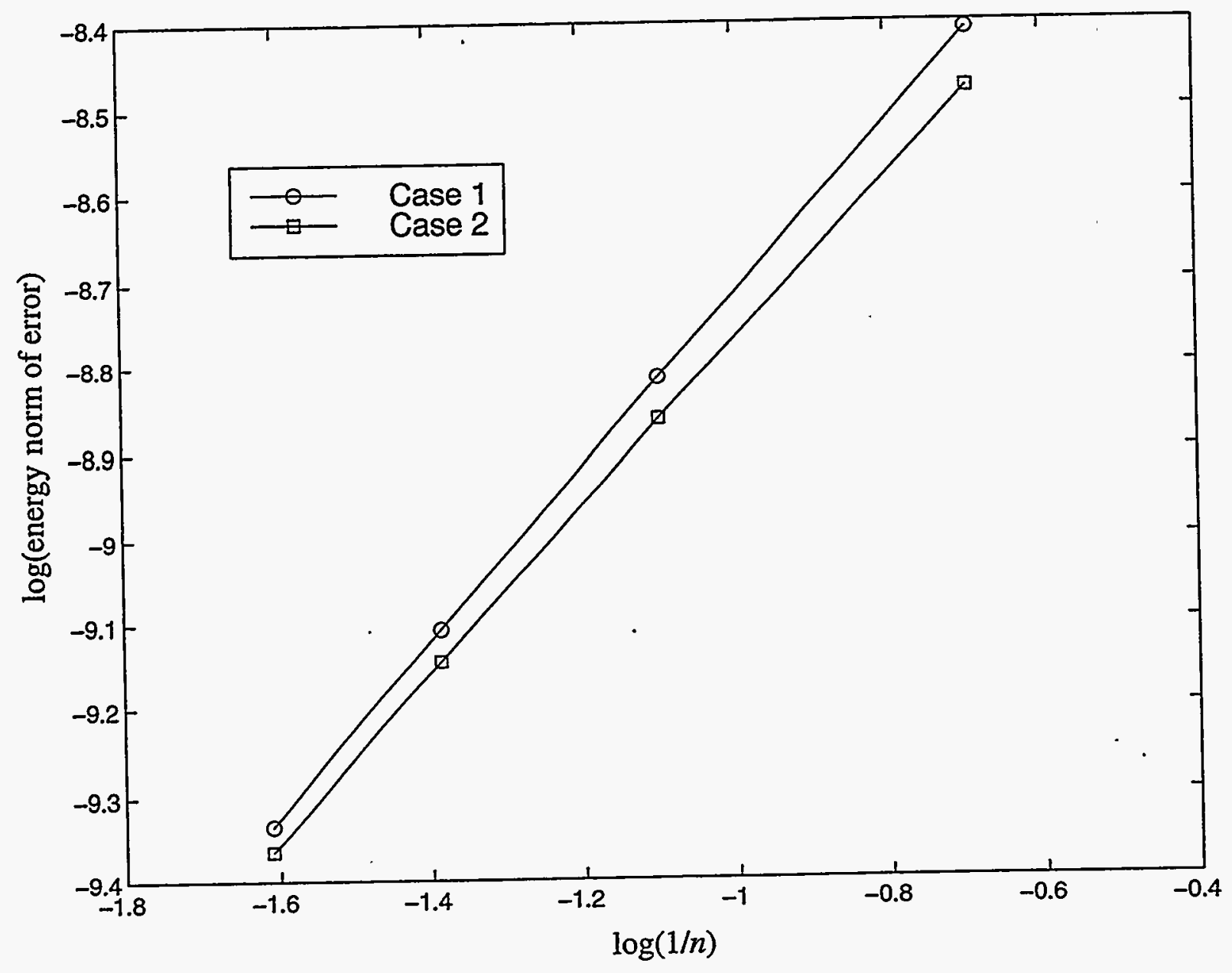

Figure 10: Energy norms of the error for Example 3.3 obtained using the present method. 


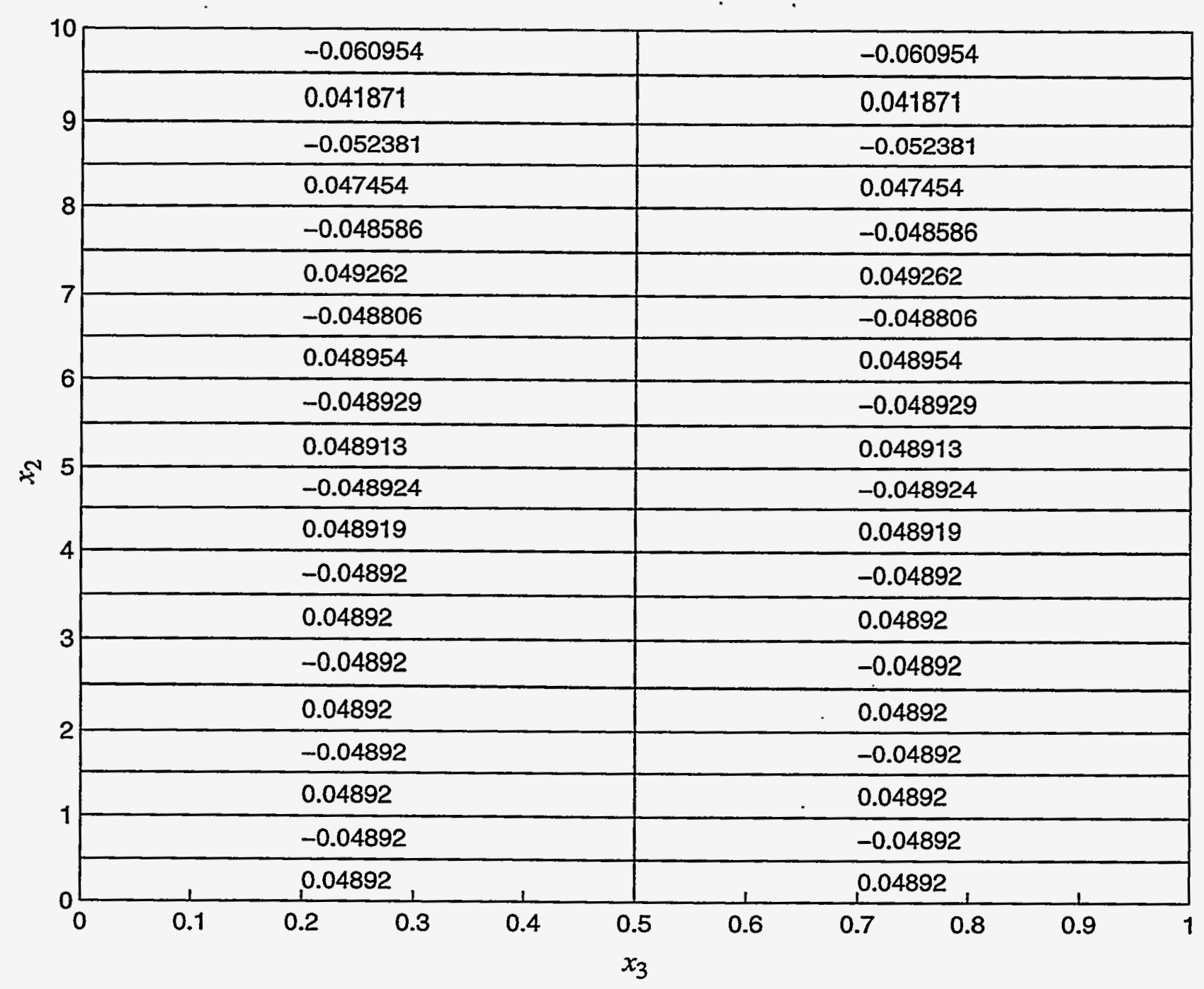

Figure 11: Stress component $\sigma_{12}$ at centroids of elements with faces on the slave surface for Case 1 of Example 3.3. 


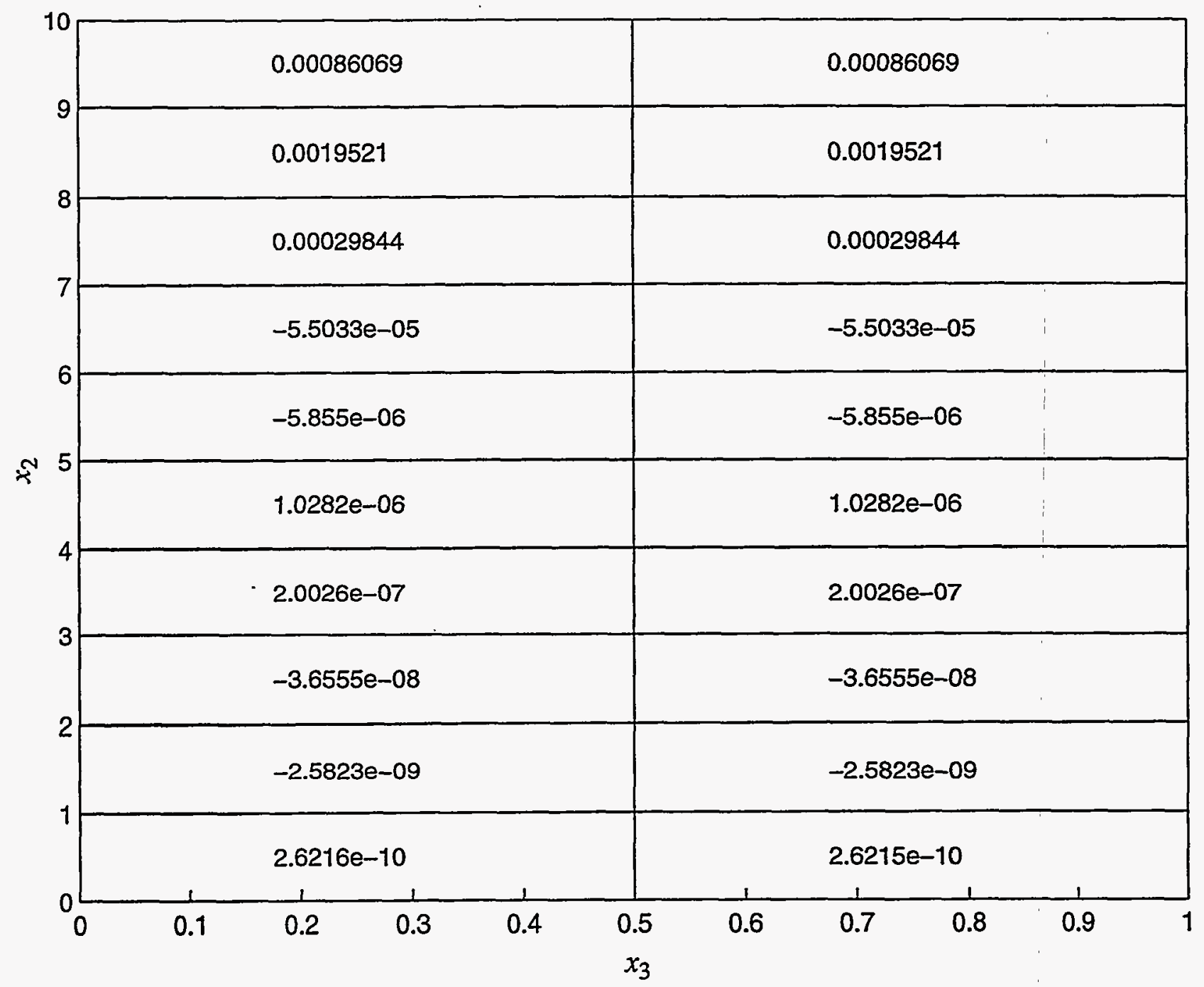

Figure 12: Stress component $\sigma_{12}$ at centroids of elements with faces on the slave surface for Case 2 of Example 3.3. 


\section{Internal Distribution:}

1 MS 9042 E. P. Chen, 8742

1 MS 9042 R. E. Jones, 8742

1 MS 9042 L. E. Voelker, 8742

1 MS 9042 L. I. Weingarten, 8742

1 MS 9405 P. E. Nielan, 8743

1 MS 9042 M. L. Chiesa, 8743

1 MS 9405 J. F. Lathrop, 8743

1 MS 0828 F. J. Mello, 9104

1 MS 0826 D. K. Gartling, 9111

1 MS 0835 R. J. Cochran, 9113

1 MS 0443 H. S. Morgan, 9117

1 MS 0443 S. W. Attaway, 9117

1 MS 0443 M. L. Blanford, 9117

1 MS 0443 K. H. Brown, 9117

10 MS 0443 M. W. Heinstein, 9117

10 MS 0443 S. W. Key, 9117

1 MS 0443 J. R. Koteras, 9117

1 MS 0443 J. W. Swegle, 9117

1 MS 0443 C. M. Stone, 9117

1 MS 0836 J. R. Stewart, 9121

1 MS 0836 L. M. Taylor, 9121

1 MS 0819 K. G. Budge, 9231

1 MS 0819 J. S. Peery, 9231

1 MS 0819 J. R. Weatherby, 9231

1 MS 0443 J. Jung, 9135

1 MS 0441 R. W. Leland, 9226

1 MS 0441 S. A. Mitchell, 9226

1 MS 0439 D. R. Martinez, 9234

10 MS 0439 C. R. Dohrmann, 9234

1 MS 0439 C. W. Fulcher, 9234

1 MS 0439 G. M. Reese, 9234

1 MS 0439 D. J. Segalman, 9234

1 MS 9018 Central Technical Files, 8940-2

2 MS 0899 Technical Library, 4916

1 MS 0619 Review \& Approval Desk, 15102 For DOE/OSTI 


\section{External Distribution:}

Dr. Claus C. Hoff

MacNeal-Schwendler Corp.

815 Colorado Boulevard

Los Angeles, CA 90041-1777

Dr. Cees Florie

MacNeal-Schwendler Benelux B. V.

Groningenweg 6

2803 PV Gouda

The Netherlands

Dr. Dennis P. Flanagan

Hibbitt, Karlsson \& Sorenson, Inc. 1080 Main Street

Pawtucket, RI 02860-4847

Mr. Roger Keene

Hibbitt, Karlsson \& Sorenson (UK), Ltd.

Genesis Center

Science Park South, Birchwood

Warington, Cheshire WA37BH

United Kingdom

Professor Ted Belytschko

Department of Mechanical Engineering

Northwestern University

2145 Sheridan Road

Evanston, II 60208-3111

Prof. K. C. Park

Center for Space Structures \& Controls

Campus Box 429

University of Colorado

Boulder, CO 80309

Dr. Jeffrey Weiss

Orthopedic Biomechanics Institute

5848 South 300 East

Salt Lake City, UT 84107
Professor Philippe H. Geubelle (3 copies)

Department of Aero and Astro Engr

University of Illinois

306 Talbot Laboratory

104 South Wright Street

Urbana, IL 61801-2935

Dr. David Chou

CSAR Corporation

28035 Dorothy Drive

Agoura Hills, CA 91301

Professor Tod A. Laursen

Department of Civil and Enviro Engr

Duke University

Box 90287

Durham, NC 27708-0287

Mr. Ken Walker

MacNeal-Schwendler Corp.

1000 Main Street, Suite 190

Grapevine, TX 76051 